volume 2

ISSUE 2

2014 Dicembre

Editor in Chief Roberto Nardi

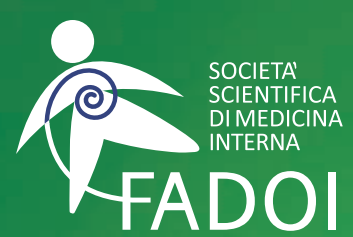

FEDERAZIONE

DELLE ASSOCIAZIONI

DEI DIRIGENTI

OSPEDALIERI

INTERNISTI

\title{
QuADERNI
}

dell'Italian Journal of Medicine

A Journal of Hospital

and Internal Medicine

The official journal of the Federation of Associations of Hospital Doctors on Internal Medicine (FADOI)

\section{Medicina Interna e Clinical Governance: quali proposte per il prossimo futuro?}

Guest Editors: A. Montagnani, G. Tintori, R. Nardi 
Mauro Campanini, Novara, Italy

\section{PRESIDENTE ELETTO}

Andrea Fontanella, Napoli, Italy

\section{PAST PRESIDENT}

Carlo Nozzoli, Firenze, Italy

\section{SEGRETARIO}

Giuseppe Augello, Canicattì (AG), Italy

\section{TESORIERE}

Dario Manfellotto, Roma, Italy

DIRETTORE RIVISTA

ITALIAN JOURNAL OF MEDICINE

Roberto Nardi, Bologna, Italy
RESPONSABILE RAPPORTI SOCIETÀ SCIENTIFICHE E ISTITUZIONI

Antonino Mazzone, Legnano (MI), Italy

RESPONSABILE SISTEMA GESTIONE QUALITÀ

Franco Berti, Roma, Italy

RESPONSABILE SITO NAZIONALE

Francesco Cipollini, Ascoli Piceno, Italy

DELEGATO FADOI ITALIAN STROKE ORGANIZATION

Michele Stornello, Siracusa, Italy

COMMISSIONE FADOI GIOVANI

Paola Gnerre, Savona, Italy

\section{Fondazione FADOI - Organigramma}

PRESIDENTE

Giorgio Vescovo, Padova, Italy

\section{COORDINATORE}

Domenico Panuccio, Bologna, Italy

\section{DIRETTORE SCIENTIFICO}

Gualberto Gussoni, Milano, Italy

DIRETTORE DIPARTIMENTO PER LA FORMAZIONE ED AGGIORNAMENTO

Mauro Silingardi, Guastalla (RE), Italy
DIRETTORE DIPARTIMENTO PER LA RICERCA CLINICA

Giancarlo Agnelli, Perugia, Italy

\section{CONSIGLIO DI AMMINISTRAZIONE}

Consigliere

Consigliere

Consigliere

Consigliere

Consigliere

Consigliere

Consigliere

Consigliere

Consigliere
Paolo Arullani, Roma, Italy

Davide Croce, Castellanza (VA), Italy

Francesco D'Amore, Roma, Italy

Salvatore Di Rosa, Palermo, Italy

Sandro Fontana, Biella, Italy

Ranieri Guerra, Roma, Italy

Ido Iori, Reggio Emilia, Italy

Giovanni Mathieu, Pinerolo (TO), Italy

Cecilia Politi, Isernia, Italy 


\section{QUADERNI - Italian Journal of Medicine}

INTRODUZIONE

Medicina Interna oggi: introduzione

M. Campanini

\section{RASSEGNE}

Cos'è la Medicina Interna?

M. La Regina, P. Gnerre

La Medicina Interna deve garantire una buona qualità di assistenza alle persone anziane ospedalizzate

G. Iosa

Lean organization e learning organization a confronto

per la Medicina Interna

D. Croce

Clinical governance: ultima spiaggia per salvare la sanità pubblica?. . .

È ancora possibile una reale alleanza tra clinici e gestori per una Medicina Interna ospedaliera sostenibile?. . . . . . . . . . S. De Carli

La cultura internistica come visione di un auspicato cambiamento in medicina e nella organizzazione ospedaliera:

l'esperienza di due realtà toscane.

G. Landini, G. Panigada, L. Masotti, I. Chiti

Indicatori di qualità nella Medicina Interna ospedaliera: solamente durata di degenza, ricoveri inappropriati, mortalità intraospedaliera e tasso di re-ricovero a 30 giorni? .... . E. Foglia, D. Croce

Medicina Interna e rischio clinico: criticità e possibili correttivi . . .

G. Chesi, F. Dall'Orto, P. Ragni, R. Nardi

Nuovi assetti organizzativi in area medica: aspetti gestionali clinici

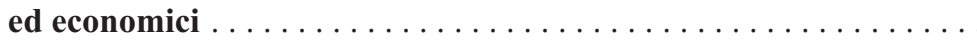
I. Stefani, A. Mazzone

Troppe procedure

Medicina Interna e wise medicine: le proposte FADOI, le prospettive assistenziali e le ricadute gestionali.

G. Panigada, I. Chiti, A. Fortini, L. Masotti, N. Napoli

Il percorso del tromboembolismo venoso nell'ospedale per intensità di cure: paradigma di wise medicine 


\section{QUADERNI - Italian Journal of Medicine}

Governo clinico e Medicina Interna: quali proposte per il prossimo

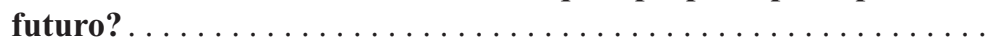

R. Risicato

Potenzialità dell'audit clinico in Medicina Interna:

lo studio DDIMA . . . . . . . . . . . . . . . . . . . . 158

M. Gambacorta, in collaborazione con il DDIMA Group

Conclusioni: Medicina Interna domani . 


\title{
Medicina Interna oggi: introduzione
}

\author{
Mauro Campanini \\ Medicina Interna 2 - Dipartimento Medico, AOU Maggiore della Carità, Novara, Italia
}

La Medicina Interna è la specialità più rappresentata sul territorio nazionale. Ogni ospedale, da quelli metropolitani di maggiori dimensioni e di elevata specializzazione a quelli periferici con minore dotazione di posti letto e di specialità, presenta nel proprio interno una o più strutture complesse di medicina. $\mathrm{Si}$ stima che in Italia gli internisti siano circa 11.000. Nonostante questo dato numerico rilevante e che non ha eguali nelle altre specialità mediche e chirurgiche, spesso risulta difficile sia alla popolazione che agli organi decisori nazionali e regionali identificare le nostre competenze; siamo in poche parole difficilmente identificabili in uno specifico contesto. Questo è il risultato dell'organizzazione sanitaria del recente passato che aveva favorito lo sviluppo di specialità mediche, gemmazione della Medicina Interna, dedicate al singolo organo od apparato, se non anche ad una singola malattia, rendendole pertanto facilmente identificabili e relegando la Medicina Interna ad un ruolo meno definito e spesso marginale. Questo errore concettuale ha visto il proliferare di sotto-specialità mediche, con un'organizzazione sanitaria più orientata alle competenze dei professionisti che ai bisogni dei malati, con la conseguenza di parcellizzare le cure perdendo di vista il principale obiettivo che è l'approccio complessivo ai bisogni sanitari della persona. Questo è il compito che è in grado di assolvere la medicina interna avvalendosi delle altre competenze specialistiche quando necessario e non viceversa. In questi ultimi anni però, in rapporto al cambio epocale dell'epidemiologia dei pazienti, in parte correlato al processo di invecchiamento della popolazione, che ha portato ad avere pazienti sempre più complessi e portatori di polipatologie vede la Medicina Interna, recuperare un

Corrispondente: Mauro Campanini, Medicina Interna 2 Centro Trombosi - Dipartimento Medico, AOU Maggiore della Carità, Corso Mazzini 18, 28100 Novara, Italia.

Tel.: +39.0321.3733310.

E-mail: mauro.campanini@maggioreosp.novara.it

Articolo pubblicato secondo la Creative Commons Attribution NonCommercial 3.0 License (CC BY-NC 3.0).

(C) Copyright M. Campanini, 2014

Licensee PAGEPress, Italy

QUADERNI - Italian Journal of Medicine 2014; 2:71-72 ruolo centrale nell'organizzazione sanitaria del nostro paese, in Europa e nel mondo. Ma come conseguenza delle variazioni delle caratteristiche dei pazienti anche il ruolo e le competenze dell'internista sono cambiate negli ultimi anni, attraverso l'acquisizione di nuove capacità tecniche, strumentali e gestionali. È per questo che il nostro Editor-in-Chief, dott. Roberto Nardi, con la sua visione illuminata, ha pensato di dedicare una monografia sul ruolo e significato della medicina interna oggi. Come Società Scientifica sentiamo il dovere e la necessità di approfondire questi temi nell'ottica di una migliore definizione del nostro ruolo nell'ambito del Sistema Sanitario Nazionale per fare sì che gli organi decisori, Ministero della Salute ed Assessorati regionali, comprendano a fondo il ruolo insostituibile della medicina interna, la valorizzino, ponendola al centro del processo clinico assistenziale.

Ma qual è il ruolo della Medicina Interna oggi?

Noi siamo in primo luogo i medici della complessità intesa però nelle sue due accezioni. Da un lato espressione di difficoltà alle diagnosi di una singola patologia e dall'altro dei problemi connessi con la multimorbidità e le comorbidità del singolo paziente. Il primo aspetto è cruciale e la Medicina Interna svolge un ruolo insostituibile nel panorama sanitario. Noi siamo infatti gli specialisti delle diagnosi complesse e difficili. Il paziente con sintomi spesso usuali, quali la febbre e l'astenia può essere affetto da patologie rare, complesse, difficili da diagnosticare e che richiedono nella loro formulazione ed articolazione una coltura medica vasta e profonda supportata da una consistente esperienza clinica. Questo dato affonda le sue radici nella storia quando Erodoto afferma che gli antichi Egizi avevano un medico dedicato alle malattie nascoste, poco associate a manifestazioni esterne o con cause profondamente oscure, le malattie interne.

Nella seconda accezione di complessità ancora una volta la Medicina Interna è il naturale gestore di questa condizione clinica, intesa non solo, come spesso accade in letteratura ed in modo riduttivo come sinonimo di multimorbidità e comorbidità ma come approccio sistematico, strutturato per priorità cliniche e dalla conoscenza delle interazioni tra patologie e terapie con scelte diagnostiche e terapeutiche, difficili, articolate e frutto di una coltura profonda supportata dall'esperienza maturata fin dalla scuola di specializzazione. Però per affrontare questi aspetti clinici in modo otti- 
male e moderno l'internista ha sviluppato nuove capacità diagnostiche e terapeutiche come la ventilazione non invasiva e l'ecografia bedside ed internistica. Questo ha permesso alla Medicina Interna di affrontare la cura del paziente critico anche attraverso la costituzione di aree dedicate con personale medico ed infermieristico addestrato. Pertanto si identifica una nuova figura dello specialista in Medicina Interna che affianca alle capacità colturali ed olistiche anche competenze tecniche evolute. Pertanto come società scientifica abbiamo strutturato la nostra offerta formativa anche su questi aspetti pratici con metodiche innovative come quella del paziente virtuale.

L'internista oggi è il medico della persona che affronta i suoi problemi clinici ed assistenziali con l'abitudine al ragionamento clinico complesso ma che utilizza le più moderne ed attuali pratiche diagnostiche e terapeutiche.

L'internista moderno non può però esimersi dallo sviluppare anche conoscenze gestionali che consentono di organizzare percorsi diagnostici e di cura nel rispetto dell'appropriatezza clinica e dell'attenzione al consumo di risorse. Le conoscenze approfondite di clinical competence e clinical governance permettono di ottenere capacità tecniche ed organizzative in modo da essere presenti in modo coerente nel sistema sanitario attuale che, con la continua richiesta di contenimento dei costi, richiede di effettuare prestazioni sanitarie con un miglior rapporto di efficacia/efficienza. Tali competenze inoltre apportano le capacità per costruire nuovi modelli organizzativi che contemplino percorsi clinici strutturati che si snodano dalle aree critiche fino al territorio, nell'ottica di offrire una adeguata continuità assistenziale.

La medicina interna è in grado di svolgere questi compiti e quindi è la specialità della persona malata e non della malattia, potendosi integrare in modo ottimale anche con il territorio nell'ottica della continuità assistenziale. Proprio per questa visione del malato come persona malata l'internista svolge e dovrà svolgere sempre di più anche la figura di hospitalist nelle realtà chirurgiche in modo da offrire anche negli ambienti sanitari di area non medica la migliore assistenza pre- e post-chirurgica.

In conclusione, riprendendo una definizione della Società Europea di Medicina Interna (EFIM) anche oggi la Medicina Interna è sempre di più la pietra angolare del Sistema Sanitario. 


\title{
Cos’è la Medicina Interna?
}

\author{
Micaela La Regina, ${ }^{1}$ Paola Gnerre ${ }^{2}$ \\ ${ }^{1}$ SC Medicina Interna, Presidio Ospedaliero Unico del Levante Ligure, La Spezia; ${ }^{2}$ SC Medicina Interna II, Ospedale San Paolo, \\ Savona, Italia
}

\section{RIASSUNTO}

Definire la medicina interna come disciplina e come struttura operativa non è facile, neppure per gli addetti ai lavori. Discipline che traggono il loro nome dall'organo o apparato (es. pneumologia, cardiologia,...), dall'epoca della vita (pediatria, geriatria,...) o dal luogo di intervento (medicina d'urgenza, ecc.), sono molto più facilmente identificate da sanitari e laici. Eppure la medicina interna è il tronco dal quale sono gemmate, negli anni, tutte le specialità non chirurgiche. Nel mondo moderno, la complessità è divenuta tale che l'eccessiva specializzazione, perseguita fino a pochi anni fa, attualmente, inizia a rappresentare un limite, soprattutto nelle funzioni di coordinamento, per via della frammentazione del sapere. In economia e in informatica, oggigiorno sono più richiesti specialisti cosiddetti wide-ranging o di ampio respiro, come per l'appunto in medicina può essere considerato l'internista, un esperto nella cura delle malattie degli adulti, che si prende cura del paziente nel suo insieme e per tutta la vita.

\section{Introduzione}

Dare una definizione di medicina interna non è cosa semplice. Neanche per un medico. E paradossalmente neppure per un'internista. La difficoltà si amplifica quando la risposta è diretta a un laico.

Il termine medicina interna ha origini oscure. Fu probabilmente usato per la prima volta in Italia, nel 1839, al Congresso degli Scienziati Italiani a Pisa, per distinguerla dalla dermatologia, all'epoca definita $m e-$ dicina esterna.

La scarsa eloquenza del termine e l'identificazione per esclusione (la medicina interna si occupa di ciò che

Corrispondente: Micaela La Regina, SC Medicina Interna, Presidio Ospedaliero Unico del Levante Ligure, Ospedale S. Andrea, via Vittorio Veneto, 19124 La Spezia, Italia. Tel.: +39.338.2393805

E-mail: micaela.laregina@asl5.liguria.it

Parole chiave: medicina interna, internista, specialista, wideranging specialist, clinical competence.

Ringraziamenti: gli autori ringraziano, con affetto e riconoscenza, i dottori Sandro Fontana e Salvatore Di Rosa, fondatori e past presidents della FADOI, per le preziose informazioni sulla storia della medicina interna in Italia.

Articolo pubblicato secondo la Creative Commons Attribution NonCommercial 3.0 License (CC BY-NC 3.0).

C Copyright M. La Regina e P. Gnerre, 2014

Licensee PAGEPress, Italy

QUADERNI - Italian Journal of Medicine 2014; 2:73-79 non è di pertinenza della dermatologia, della chirugia, della ginecologia, ecc.) hanno sicuramente contribuito all'offuscamento della disciplina durante la crescente specializzazione e super specializzazione degli anni '70-80-90. Discipline che traggono il loro nome dall'organo o apparato (es. pneumologia, cardiologia,...), dall'epoca della vita (pediatria, geriatria,...) o dal luogo di intervento (medicina d'urgenza, ecc.), sono molto più facilmente identificate da sanitari e laici.

Esistono in letteratura diverse definizioni di internista. Tra le più moderne, citiamo:

i) la definizione della European Union of Specialists: internist is a physician trained in the scientific basis of medicine, who specialises in the assessment, diagnosis and management of general medical problems, atypical presentations, multiple problems or system disorders. The physician is skilled in the management of acute unselected medical emergencies and the management of patients in a holistic and ethical way, considering all psychological as well as medical factors for enhancing quality of life. The physician values the continuing of care of all patients irrespective of the nature of patient's complaint and is committed to lifelong continued professional development. The physician practices clinical audit and evidencebased medicine. The physician function in a number of roles, including clinical counselling, educating, leading and managing; ${ }^{1}$

ii) la definizione del Royal College of Physicians and Surgeons of Canada: an internist is a specialist trained in the diagnosis and treatment of a broad range of diseases in adults involving all organ and systems, and is proficient in the medical manage- 
ment of patients who have undifferentiated or multi-system disease processes. An internist cares for hospitalized and ambulatory patients and may play a role in teaching or research, ${ }^{2}$

iii) e la definizione dell'American College of Physicians: Doctors of internal medicine are experts in adults, they care for the whole patient and for his/her life. ${ }^{3}$

L'American College of Physicians ha promosso addirittura una campagna informativa rivolta alla comunità su chi è l'internista e di cosa si occupa e per chiarire alcune frequenti confusioni fra internists e interns o fra general internists e general practitioners. In tale brochure l'internista è definito anche doctor's doctor perché chiamato a risolvere casi intricati. ${ }^{3}$

La moderna epistemologia riconosce al termine medicina interna almeno tre aspetti: la complessità, la rarità e l'olismo.

Recentemente l'internista è entrato nell'immaginario collettivo almeno come specialista dei casi intricati, grazie alla fortunata serie TV americana Doctor House.

\section{Storia}

Il termine medicina interna o meglio Innere Medizin cominciò a diffondersi in Germania nel 1880. La prima testimonianza scritta è rappresentata dagli atti del primo congresso a essa dedicato che si tenne a Wiesbaden, in Germania, dal 20 al 22 aprile del 1882 e che sancì la nascita della medicina interna come disciplina diversa dalla chirurgia e dalla ginecologia/ostetricia.

Fino al 1883 i trattati di medicina interna in Germania portavano il titolo di trattati di patologia e terapia. Solo nel 1883, Adolf Strumpell aggiunse al titolo del suo trattato der inneren krankheiten vale a dire of internal diseases, ponendosi in opposizione all'external medicine, la dermatologia, che era molto sviluppata all'epoca, a causa della sifilide.

Nel 1894 il famoso giornale Centralblatt fur klinische medicine cambiò improvvisamente il suo nome in Centralblatt fur Innere Medizin, spiegando nella prefazione che l'intenzione era di non occuparsi solo di argomenti clinici, ma anche di fornire una rassegna di qualsiasi topic nella letteratura mondiale fosse dominio della medicina interna. Successivamente, fu ribadito, a più voci, che la medicina interna non si occupava solo della descrizione clinica delle malattie, ma includeva anche lo studio delle loro basi fisiopatologiche, da cui l'aggettivo interna.

Nata in Europa, la medicina interna approdò anche negli Stati Uniti, dove le sue radici affondano nell' $A s$ sociation of American Physicians (AAP), un'associazione elitaria di internisti che vivevano sulla costa orientale (1885). Tale associazione aveva una mission educativa e pertanto si occupava di esperienze cliniche e nuove scoperte, escludendo l'etica e la politica. Al primo congresso dell'associazione, Reginald Fitz tenne la prima descrizione del quadro clinico dell'appendicite acuta. Molte altre novità scientifiche furono presentate nei congressi successivi dell'AAP. Un'associazione sorella, l'American Society for Clinical Investigation (ASCI), fu fondata nel 1909 dal Dr. Samuel Meltzer, con l'obiettivo di sviluppare e promuovere la ricerca clinica. I congressi congiunti dell'AAP e ASCI, ad Atlanta, furono per anni il riferimento accademico per la medicina americana e mondiale. Il primo a utilizzare il termine medicina interna in America fu William Osler che, nel discorso di apertura del decimo congresso, quale presidente dell'AAP, descrisse anche il training necessario per diventare internisti: 10-15 anni dopo la laurea di rigoroso lavoro, prima nei laboratori perché la patologia è la base solida della medicina interna, poi nei reparti, con poco tempo per gli amori e il matrimonio. ${ }^{4}$ Concetto ribadito anche da un altro illustre internista, italiano, Agusto Murri il quale ammonì i suoi studenti dicendo: Sappiate che qui non si vince, se non con lungo studio e grande amore. Il focus della disciplina medicina interna sulla scienza e sulla ricerca le consentirono di insediarsi, come la chirurgia, all'interno dell'ospedale.

In Italia la prima Società Scientifica di Medicina Interna nacque in occasione di un'adunanza, a Pavia, della Società Italiana di Medicina, che riuniva allora i cultori di tutte le branche della medicina e della chirurgia. Un gruppo di giovani cattedratici di Clinica Medica riconobbe la necessità di creare una Società di Medicina Interna nell'ottobre del 1887, quando nel mondo non esisteva che la Deutscher Verein für Innere Medizin. Il primo Congresso ebbe luogo a Roma l'anno dopo (1888).

Negli anni successivi, fiorirono le società scientifiche di medicina interna nel resto del mondo: la Società Giapponese nel 1902; la Danese nel 1916; l'Argentina nel 1920; la Svizzera nel 1932; la Spagnola nel 1952; la Cubana nel 1957; la Paraguaiana e la Colombiana nel 1975, ed infine quella Francese nel 1979.

Sul piano internazionale, va anche ricordato che, nel 1948, a Basilea, fu fondata l'International Society of Internal Medicine, e che, nel 1969, a Strasburgo fu fondata l'Association Européenne de Médicine Interne (AEMI). Infine, nel Maggio del 1996, a Parigi, da una fusione fra la quest'ultima e il Forum of Presidents of National Societes of Internal Medicine nacque l'European Federation of Internal Medicine. I fondatori prof. Ugo Carcassi di Cagliari e prof. Jamie Merino di Alicante vollero con questo trasformare la elitaria AEMI di lingua francese in una federazione delle società nazionali, più accessile e di lingua inglese. ${ }^{5}$

Nell'ultimo ventennio del XX secolo, infine, anni di grande cambiamento come mai prima nel campo sociale, delle comunicazioni e della tecnologia, in Italia, 
alcuni internisti ospedalieri presenti in quegli anni nel consiglio direttivo della Società Italiana di Medicina Interna cercarono di rappresentare i problemi della medicina interna ospedaliera (necessità di valorizzare e utilizzare la grande casistica afferente agli ospedali per fare ricerca clinica, necessità formativa adeguata ai nuovi tempi che tenesse conto delle nuove tecnologie, delle nuove tecniche comunicative, della complessità dei malati internistici, della necessità di imparare sul campo) senza ottenere risposte. Pertanto, nel 1994, dopo un lungo lavoro organizzativo che fece nascere associazioni regionali nelle quali gli internisti ospedalieri trovarono un luogo di confronto e di presa di coscienza delle loro peculiari problematiche, nacque prima la FAPOI, Federazione delle Associazioni dei Primari Ospedalieri Internisti, poi la FADOI che riunì tutti i dirigenti ospedalieri internisti, non solo i primari.

Attualmente FADOI è una federazione di 18 associazioni regionali e conta oltre 2000 iscritti $(30 \%$ under 40). FADOI e SIMI hanno in corso di definizione un consenso congiunto ai fini di una visione politico-istituzionale condivisa.

\section{La Medicina Interna nell'ultimo ventennio}

Non vi sono dubbi che gli anni ' 90 hanno condotto a un rapido e sostanziale cambiamento della pratica della medicina interna, in tutto il mondo.

Negli Stati Uniti, gli Hospitalists - medici dedicati esclusivamente alla cura del paziente ospedalizzato, prevalentamente specializzati in medicina interna, dopo aver dimostrato di migliorare efficacia ed efficienza delle strutture in cui lavorano, sono diventati leaders nell'ambito della qualità, della sicurezza, della tecnologia informatica, delle cure palliative, dell'istruzione medica, ecc.. Sempre più studenti scelgono un training in medicina interna e/o hospital medicine per l'enorme sviluppo che quest'ultima ha conosciuto - da poche decine a decine di migliaia di hospitalists in America in soli 10 anni - configurandosi come la specialità medica a più rapida crescita della storia. ${ }^{6}$

In tutti i Paesi occidentali, la Medicina Interna, ha progressivamente modificato la sua attività in rapporto alle variazioni epidemiologiche (allungamento della vita media e incremento delle malattie cronico-degenerative) e socio-culturali (shift dalla famiglia alla società dell'assistenza alla cronicità) che, com'è noto, condizionano le richieste di salute. Attualmente, i reparti di Medicina Interna rappresentano la struttura che meglio può rispondere alle esigenze di pazienti acuti, con multimorbidità e spesso anche con criticità sociali.

In questo mutato contesto, la medicina interna: i) gestisce diagnosi e terapia di sempre più pazienti polipatologici, complessi e fragili; ii) offre risposte efficaci a numerose e variegate necessità di assistenza con l'ampiezza delle sue competenze e l'autonomia in talune tecnologie (es. ecografia, ventilazione non invasiva); iii) indirizza a un appropriato utilizzo delle funzioni specialistiche ad alta tecnologia consentendone la concentrazione territoriale; iv) offre il setting assistenziale più adatto alla fase della storia clinica del paziente, dall'ambulatorio/day service (DS)/day hospital (DH), al ricovero in degenza ordinaria o in area critica e fino alle aree per post-acuti; v) fornisce supporto anche nei processi di cura chirurgici, sia nelle fasi d'inquadramento pre-intervento che nelle fasi di complicanza postintervento, sia in regime di ricovero che ambulatoriale.

In alcune realtà nazionali ed internazionali, sta utilizzando modelli organizzativi dinamici incentrati sul paziente, come ad esempio il modello organizzativo per intensità di cure, in cui i pazienti sono allocati non per specialità, ma per severità di malattia e richiesta assistenziale. Dati della letteratura indicano che la popolazione dei ricoverati in Medicina Interna comprende una quota del $10 \%$ circa di soggetti, identificabili con appositi score prognostici (MEWS, NEWS, ecc.), richiedenti controlli medici più frequenti e assistenza infermieristica superiore a quella fornita in una degenza tradizionale. ${ }^{7}$ Nei paesi anglosassoni queste aree, cosiddette High Dependency Units, sono nate dalla necessità delle terapie intensive di ridurre i posti letto e selezionare più rigorosamente i pazienti afferenti ad esse, nel nostro paese, prevalentemente dal riconoscimento da parte delle strutture di medicina interna di una maggiore richiesta assistenziale da parte di taluni pazienti più instabili.

\section{Generalisti versus specialisti}

Oltre 2000 anni fa, il poeta greco Archiloco scrisse: la volpe conosce molte cose, mentre il riccio ne conosce una sola, grande, e aprì pionieristicamente il dibattito su generalisti (le volpi) e specialisti (i ricci).

Nel 1953 Isaiah Berlin, grande pensatore del Novecento, riprese quest' antinomia per dividere gli scrittori e, gli individui in generale, in due categorie: Esiste un grande divario tra coloro, da una parte, che riferiscono tutto a una visione centrale, a un sistema più o meno coerente o articolato, con regole che li guidano a capire, a pensare e a sentire - un principio ispiratore, unico e universale, il solo che può dare un significato a tutto ciò che essi sono e dicono - , e coloro, dall'altra parte, che perseguono molti fini, spesso disgiunti e contraddittori. ${ }^{8}$

Nei decenni successivi al saggio di Berlin, gli specialisti e i sub-, ultra-, iper-specialisti hanno dominato il mondo accademico e quasi tutti gli ambiti professionali, scalando posizioni sempre più alte. Per fare carriera, era utile specializzarsi sempre di più. Tuttavia, per varie ragioni, l'era degli specialisti è entrata in una fase di declino. Vivere in un mondo come il nostro fortemente globalizzato e interconnesso vuol dire 
che ambiti apparentemente lontani fra loro possono di fatto influenzarsi a vicenda, richiedendo una visione ampia per la loro gestione. Gli specialisti, invece, si muovono all'interno di ambiti molto ristretti e applicano formule standard a situazioni spesso non ben definite, risultando poco flessibili e poco efficaci nel mondo attuale. Esistono dati convincenti che i generalisti siano più adatti a navigare nell'incertezza, nella complessità e nelle contrapposizioni del mondo del lavoro presente. Il professor Philip Tetlock ha condotto uno studio di oltre 20 anni su 284 professionisti. Egli ha chiesto loro di prevedere il verificarsi di vari eventi sia all'interno che al di fuori del loro ambito di specializzazione. Analizzando oltre 80.000 previsioni ha trovato che gli esperti erano meno accurati dei non esperti nel predire gli eventi nel loro ambito di specializzazione, ${ }^{9}$ concludendo appunto che è meglio affidarsi alle volpi, categorizzate da Berlin.

In quest'attualissima disputa fra generalisti e specialisti, l'internista si colloca in posizione equidistante fra gli uni e gli altri, configurandosi come un wideranging specialist, letteralmente specialista di ampio respiro rispetto agli specialisti d'organo che invece risultano hyper-focused specialists. ${ }^{10}$

Il wide-ranging specialist è più di un semplice generalista, come può essere considerato il medico di medicina generale, che ha un'ampia conoscenza di base, ma nessuna conoscenza approfondita. L'internista, difatti, ha una più ampia conoscenza di base e conoscenze più approfondite in vari ambiti. Contemporaneamente, è più di un semplice specialista le cui conoscenze e la cui azione si concentrano su un singolo ambito ( $h y$ perfocused), al punto che è molto efficace in quell'am- bito, ma in nessun altro e, addirittura, può arrivare a non riuscire a dialogare con altri specialisti, ignorando completamente il loro ambito di azione (Figure 1 e 2).

Breadth and depth of knowledge sono uno dei core values della medicina interna secondo Eric Larson et al. ${ }^{11}$ L'internista è nel cosiddetto sweet spot tra i due estremi del generalista che paradossalmente rischia di sapere sempre meno di tutto fino a non sapere niente di tutto e dell'ultraspecialista che invece rischia di sapere sempre di più ma di meno argomenti fino a sapere tutto di niente. A metà fra generalisti e ultraspecialisti, l'internista gode dei vantaggi di entrambi, senza soffrire dei loro svantaggi.

Nel mondo dell'economia i wide-ranging specialists sono ricercati perché sono in grado di muoversi in più ambiti e hanno sufficienti conoscenze in ciascun ambito per discutere e attuare strategie di produzione. Gli hyper-focused specialists, infatti, risultano più adatti come esecutori di strategie e processi, ideati e coordinati dai primi. Il che trasferito nelle organizzazioni sanitarie supporta la figura dell'internista come regista dei percorsi diagnostico-terapeutici ospedalieri ed extra-ospedalieri, perché in grado di affrontare diversi problemi di salute, situazioni complesse e coordinare, se richiesto, più specialisti che, parlando linguaggi differenti, potrebbero avere difficoltà a integrarsi fra loro.

\section{Competenze della Medicina Interna}

È noto che le competenze della medicina interna variano da paese a paese e, talora, da ospedale a ospe-

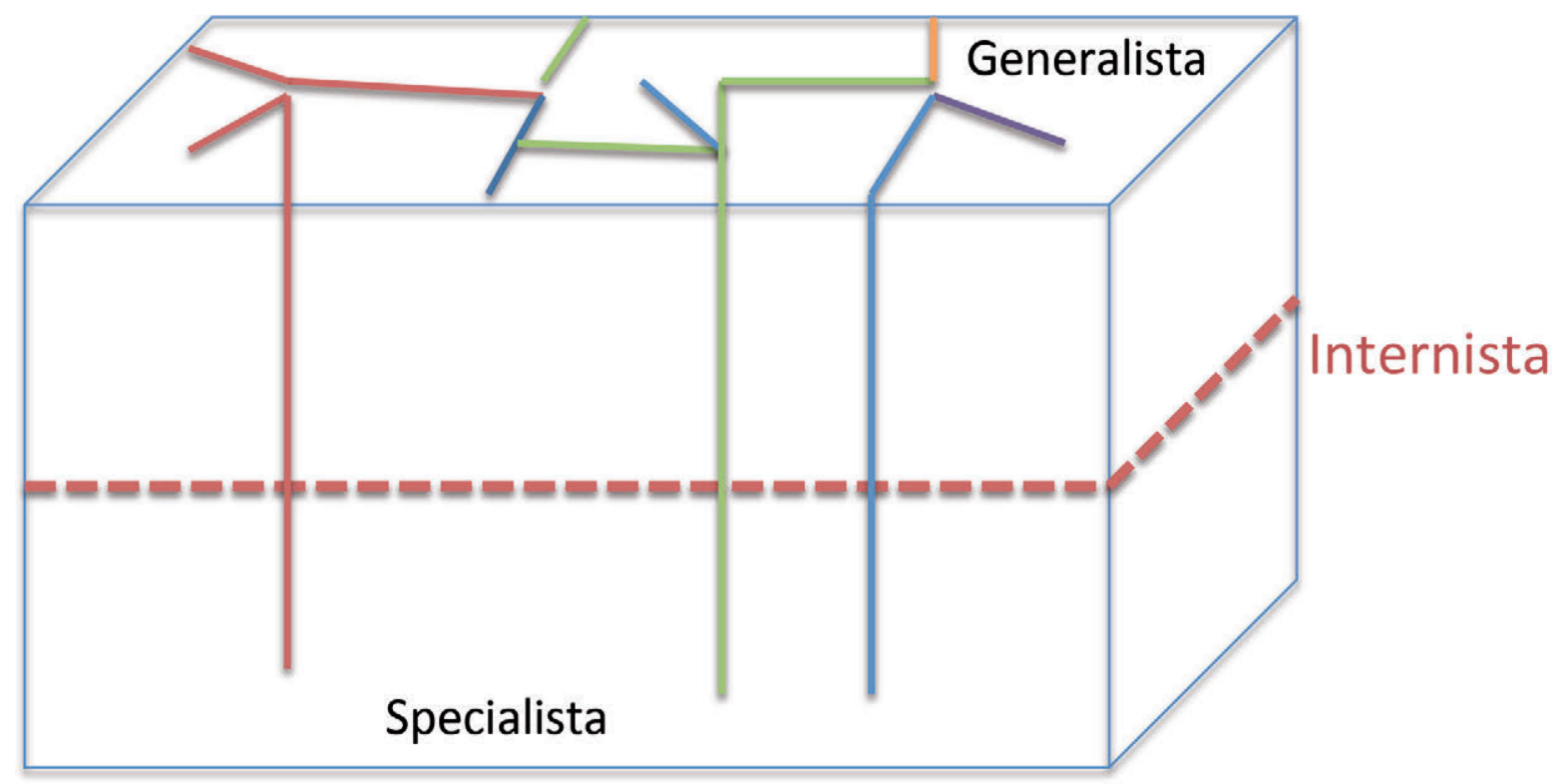

Figura 1. L'internista fra generalisti e specialisti. (Modificato da: eric.beard.com). 
dale, anche in rapporto alla domanda locale di salute. Tuttavia, in diversi paesi è stato compiuto lo sforzo di stabilire le competenze essenziali di un internista.

Negli USA, nel 1999, 1'Accreditation Council for Graduate Medical Education (ACGME) ha definito un set di 6 competenze che tutti gli specialisti in formazione in medicina interna devono conseguire per essere certificati come internisti. Tali competenze, adottate in seguito dall'American Board of Internal Medicine (ABIM), sono: i) patient care and procedural skills; ii) medical knowledge; iii) practice-based learning and improvement; iv) interpersonal and communication skills; v) professionalism; vi) system-based practice.

Per quanto riguarda l'abilità nell'esecuzione delle procedure, per la certificazione, vengono testati la conoscenza di indicazioni e controindicazioni, il riconoscimento e la gestione delle principali complicanze, la gestione del dolore e della sterilità della manovra, dei campioni ottenuti e l'interpretazione dei risultati, nonché la capacità di spiegare chiaramente al paziente la procedura per ottenerne il consenso. Le procedure richieste a un internista statunitense sono: paracentesi, advanced cardiac life support (ACLS), posizionamento di catetere arterioso, artrocentesi, posizionamento di catetere venoso centrale, prelievo venoso, prelievo arterioso, elettrocardiogramma, incisione e drenaggio di un ascesso, puntura lombare, posizionamento sondino nasogastrico, posizionamento di accesso venoso periferico, posizionamento di catetere arterioso polmonare, toracentesi, pap test e coltura cervicale. Una volta conseguita la certificazione, si entra nel Manteinance of certification (MOC) Program che prevede a lifelong learning con l'obbligo di guadagnare punti MOC attraverso una serie di corsi o prove anche su pazienti virtuali e il superamento di un esame ogni dieci anni. ${ }^{12}$

Analogamente, il Royal College of Phycians and Surgeons of Canada ha raggruppato le key and enabling competencies degli internisti in 7 domini: i) medical experience; ii) communication; iii) collaboration; iv) management; v) healthy advocation; vi) scholarship; vii) professionalism.

Anch'esso prevede il conseguimento e il mantenimento della licenza di internista. ${ }^{2}$

Nel 2007 anche la Federazione Europea di Medicina Interna (EFIM) che raggruppa le società nazionali europee di medicina interna ha pubblicato le core competencies dell'internista Europeo: i) patient care; ii) medical knowledge; iii) communication skills; iv) professionalism, ethical and legal issues; v) organizational planning and service management skills; vi) academic activities - education and research. ${ }^{1}$

Nel nostro paese, la legislazione non prevede al momento la certificazione e il mantenimento della stessa, tuttavia, FADOI, in collaborazione con SDABocconi di Milano, ha iniziato da alcuni anni un lavoro di definizione e valutazione delle competenze dell'internista ospedaliero. Partendo dal progetto $\mathrm{Mi}$ nerva basato su 161.961 dimissioni dai reparti di medicina interna italiani, è stata elaborata una mappa delle competenze dell'internista. Queste sono state distinte in due categorie: le caratteristiche organizzativo/manageriali e quelle strettamente correlate alla professione medica e alla specialità. Per ciascuna competenza è previsto un livello che può essere: di base, ottimale o eccellente. Inoltre è stato introdotto

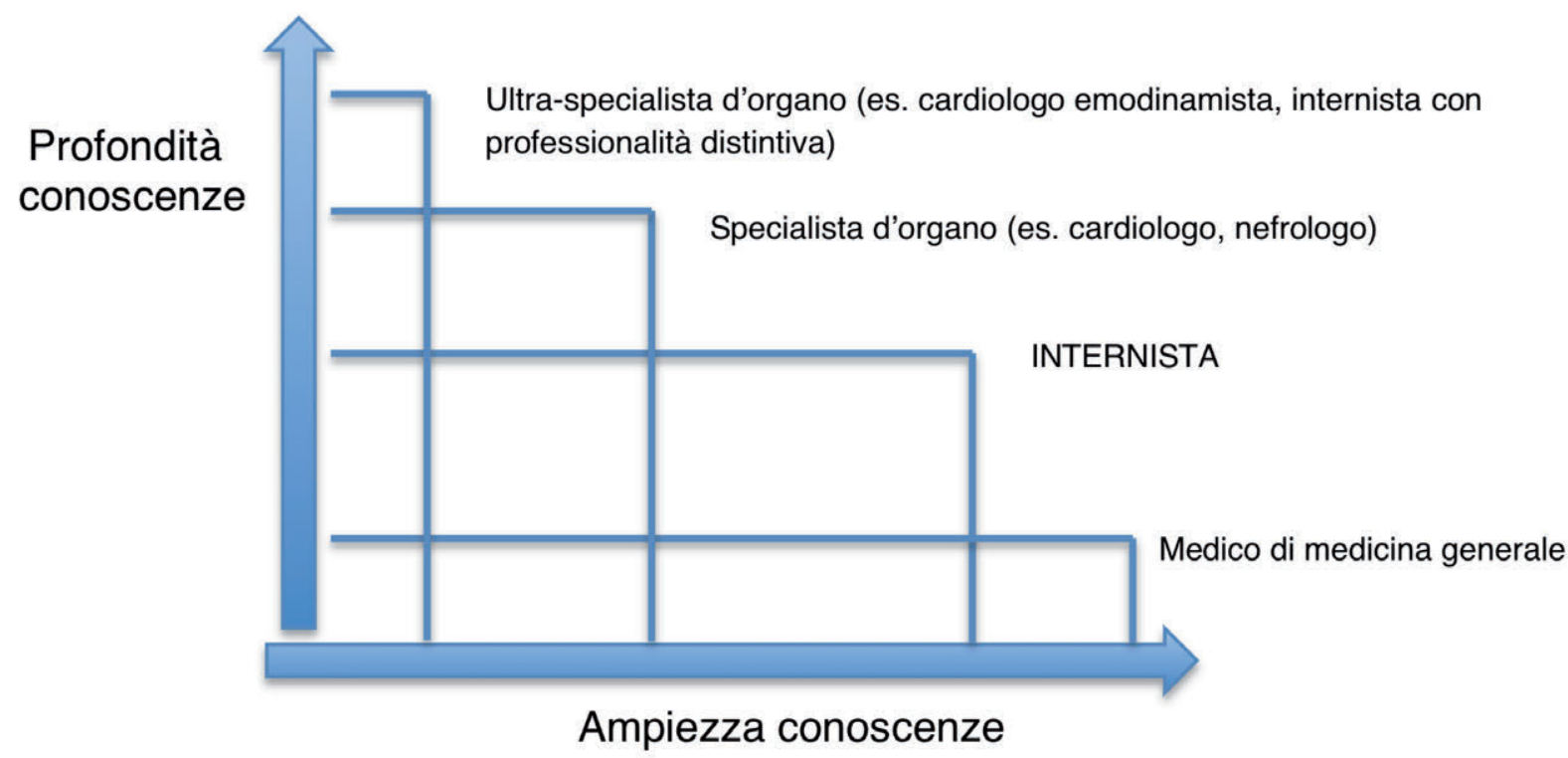

Figura 2. L'internista: ampiezza e profondità delle conoscenze. 
il concetto di professionalità distintiva per indicare la capacità di eseguire un'attività professionale, in un determinato ambito, a un livello ultra-specialistico. Nel lavoro pubblicato su un numero monografico dell'Italian Journal of Medicine nel 2011, vengono definite le prerogative distintive dell'internista: i) capacità di sintesi clinica; ii) capacità di diagnosi e terapia; iii) elevata flessibilità funzionale nella gestione di pazienti complessi con malattie sistemiche e/o multiorgano.

Viene, inoltre, proposta una griglia dove per ogni attività (clinica, gestionale, relazionale,...) vengono indicati: gli indicatori di performance, cosa bisogna conoscere e saper fare e cosa è richiesto per eccellere in detta attività. Attualmente, il gruppo di lavoro è impegnato a stabilire gli strumenti di valutazione delle competenze ai fini del conseguimento della certificazione e del suo mantenimento. ${ }^{13}$

\section{Punti di forza e di debolezza della medicina interna}

La medicina interna, come disciplina, presenta numerosi punti di forza, a favore sia dei pazienti sia delle organizzazioni sanitarie, tutti derivanti dalle sue peculiarità.

La prima peculiarità è l'utilizzo del metodo clinico, quale strumento cardine della sua pratica e del rapporto medico-paziente. Il metodo clinico è quell'insieme di atti e procedure che si realizzano nell'incontro del paziente con il medico e volto a definire e risolvere i problemi di salute del paziente, senza trascurare le conseguenze psicologiche, sociali, economiche della malattia.

La seconda è la trans-disciplinarietà, concetto introdotto in psicologia cognitiva nel 1970 da Jean Piaget, psicologo, biologo, pedagogista e filosofo svizzero e diversa dalla pluridisciplinarietà. Se la transdisciplinarietà attraversa e oltrepassa tutte le discipline specialistiche con l'obiettivo di comprendere la complessità del mondo moderno con un approccio che restituisce al sapere umano unitarietà nella diversità, ${ }^{14}$ allora si identifica pienamente con l'approccio internistico ai problemi di salute del paziente.

La terza peculiarità è la diffusione capillare dei reparti di medicina interna sul territorio.

La quarta è la flessibilità funzionale. L'internista è una figura pluri-potenziale. Vi sono internisti che hanno vinto il premio Nobel con le scienze di base, internisti che lavorano come direttori sanitari o medici di famiglia e internisti che curano pazienti critici. La tipologia d'internista che ciascuno diventa dipende dalle sue inclinazioni, ma anche dal contesto in cui lavora. ${ }^{15}$

Ne consegue che l'internista, grazie alla sua formazione specialistica di tipo diffuso, all'utilizzo del metodo clinico quale principale strumento di lavoro - che include le tecnologie, ma non si esaurisce con esse come per talune discipline ultra-specialistiche - e del suo approccio transdisciplinare, è in grado di superare la frammentazione della malattia e del paziente alla quale ha condotto l'eccessiva specializzazione. Se a questo si aggiunge la capillare diffusione sul territorio e la flessibilità funzionale è intuitivo che l'internista, soprattutto nella complessità della poli-patologia del malato moderno, rappresenta per pazienti ed organizzazioni l'alleato ideale per la progettazione, la coordinazione e l'esecuzione di numerosi percorsi diagnostico-terapeutici.

Sul rovescio della medaglia, i punti di debolezza che non mancano: i) scarsa conoscenza da parte di pazienti, familiari e decisori del significato e del ruolo dell'internista, anche a causa della poca intelligibilità del nome della disciplina; ii) riduzionismo di taluni internisti che, spesso per carenza di formazione, svolgono una funzione di indirizzo più che di presa in carico globale del paziente; iii) richiesta di un'etichetta ultra-specialistica da parte dei pazienti. Negli Stati Uniti, però, si sta registrando un'inversione di tendenza: i pazienti sono sempre meno preoccupati del nome dello specialista che li ha in cura e sempre più delle sue performances. In pratica, importa loro sempre meno che le cure vengano fornite da un internista o da un cardiologo o da un medico di medicina generale, purché sappia fare il suo lavoro e renda loro un buon servizio. ${ }^{15}$

\section{Conclusioni}

Dare una definizione di medicina interna non è un'impresa semplice. Prova ne sia che nel 1897, uno dei padri della medicina interna, espresse anch'egli questa difficoltà, scrivendo:

I wish there were another term to designate the wide field of medical practice which remains after separation of surgery, midwifery and gynecology. [....] its cultivators [...] bear without reproach the good old name physician, in contradistinction to general practitioners, surgeons, obstetricians and gynecologists.

I cambiamenti epidemiologici degli ultimi decenni offrono un'opportunità imperdibile alla medicina interna, dopo la spoliazione operata dalle discipline ultra-specialistiche fino agli anni ' 90.

Le competenze dell'internista appaiono quelle meglio adatte alla cura dei malati moderni, complessi e poli-patologici.

La chiave per il futuro della medicina interna è nelle mani degli internisti e dei pazienti, non delle organizzazioni. Se gli internisti saranno in grado di trasmettere ai pazienti il loro significato e i pazienti comprenderanno che l'internista è il medico di cui hanno bisogno, lo chiederanno alle organizzazioni e queste si modificheranno di conseguenza. 


\section{Bibliografia}

1. Palsson R, Kellet J, Lindgren S, et al. Core competencies of the European internist: a discussion paper. Eur J Intern Med 2007;18:104-8.

2. Royal College of Physicians and Surgeons of Canada. Objectives of training in the specialty of Internal Medicine, version 1.0; 2011. Available from: http://www.deptmedicine.utoronto.ca/Assets/DeptMed+Digital+Assets/C ore+Internal+Medicine+Files/rcpscobjectives.pdf

3. American College of Physicians. About Internal Medicine: What's an "Internist"? Available from: http://www. acponline.org/patients_families/about_internal_medicine/

4. Fordtran JS, Armstrong WM, Emmett M, et al. The history of internal medicine at Baylor University Medical Center, part 1. Proc Bayl Univ Med Cent 2004;17:9-22.

5. Carcassi UE, Elte JW, Davidson C. The history of the (Young) AEMI(E) and the EFIM. Eur J Intern Med 2007;18:26-30.

6. Wachter RW. The hospitalist movement 10 years later: life as a Swiss army knife. Med Gen Med 2006;8:30.

7. Cei M, Bartolomei C, Mumoli N. In-hospital mortality and morbidity of elderly medical patients can be predicted at admission by the modified early warning score: a prospective study. Int J Clin Pract 2009;63:591-5.
8. Berlin I. Il riccio e la volpe. Milano: Adelphi edizioni; 1998.

9. Tetlock PE. Expert political judgment: How good is it? How can we know? Princeton: Princeton Univ. Press; 2005.

10. Forbes Woman. The secret power of the generalist - and how they'll rule the future; 7/10/2012. Available from: http://www.forbes.com/sites/meghancasserly/2012/07/1 0/the-secret-power-of-the-generalist-and-how-theyllrule-the-future/

11. Larson EB, Fihn SD, Kirk LM, et al. The future of general internal medicine. J Gen Intern Med 2004;19:69-79.

12. American Board of Internal Medicine. Internal Medicine policies. Available from: www.abim.org/certification/policies/imss/im.aspx

13. Mazzone A, Nozzoli C, Berti F, et al. The Clinical competence in Internal Medicine. Ital J Med 2011;5 Suppl $1: 1-28$.

14. Piaget J. The epistemology of interdisciplinary relationships. In: Apostel L, Berger G, Briggs A, Michaud G, eds. Interdisciplinarity. Problems of teaching and research in universities. Paris: OECD; 1972. pp 127-139.

15. Gesensway D. What is internal medicine's future? ACP Internist 1998. Available from: www.acpinternist.org/archives/1998/03/future.htm 


\title{
La Medicina Interna deve garantire una buona qualità di assistenza alle persone anziane ospedalizzate
}

\author{
Giovanni Iosa \\ UO Medicina Interna Cesenatico, Ospedale Bufalini-Marconi-Angioloni, AUSL Romagna, Italia
}

\begin{abstract}
RIASSUNTO
Gli anziani sono tra i principali utilizzatori dei servizi sanitari e in particolare degli ospedali. Internisti e geriatri devono collaborare per migliorare le cure dell'anziano e discutere anche di modelli assistenziali innovativi. Il passo fondamentale dovrà essere il trasferimento agli internisti dell'appropriato approccio ai pazienti anziani ospedalizzati che si basa sulla comprensive geriatric assessment, una metodologia che genera un sistema di interventi e cure mediche volte a preservare l'autosufficienza e la qualità della vita. La collaborazione potrebbe iniziare definendo le competenze geriatriche essenziali per l'internista in 7 aree: i) gestione dei farmaci; ii) aspetto cognitivo, affettivo e comportamentale; iii) malattia complessa o cronica negli anziani; iv) terapie palliative e di and-life care; v) sicurezza del paziente in Ospedale; vi) transizioni di cura; vii) e gestione ambulatoriale.
\end{abstract}

\section{La Medicina Interna deve garantire una buona qualità di assistenza alle persone anziane ospedalizzate}

In tutto il mondo, l'aspettativa di vita alla nascita è in progressivo aumento e nei paesi più sviluppati ha raggiunto livelli inimmaginabili fino a pochi decenni fa. Di conseguenza, l'indice di dipendenza (il rapporto fra la popolazione di età pari o superiore ai 65 anni e quella di età compresa fra i 15 e i 64 anni) cresce costantemente, aumentando così le preoccupazioni circa la sostenibilità dei sistemi di welfare. Alcuni autori, tuttavia, autorevolmente sostengono che questa preoccupazione dovrebbe essere riconsiderata alla luce dei cambiamenti qualitativi che si sono verificati con la longevità. ${ }^{1}$

In questo scenario, la sfida per il futuro è quella di garantire che le persone possano essere in grado di invecchiare con sicurezza e dignità mantenendo il massimo grado di inserimento nei loro contesti sociali. ${ }^{2}$

Corrispondente: Giovanni Iosa, V. C. Abba 102, 47042 Cesenatico (FC), Italia.

Tel. +39.0547.674803.

E-mail: giosa@ausl-cesena.emr.it

Parole chiave: comprensive geriatric assessment, best practices assistenziali, interdipendenza e trasversalità nei processi assistenziali.

Articolo pubblicato secondo la Creative Commons Attribution NonCommercial 3.0 License (CC BY-NC 3.0).

CCopyright G. Iosa, 2014

Licensee PAGEPress, Italy

QUADERNI - Italian Journal of Medicine 2014; 2:80-82
Gli anziani sono tra i principali utilizzatori dei servizi sanitari e in particolare degli ospedali.

L'ospedalizzazione peraltro svolge un ruolo importante nella traiettoria che porta alla compromissione funzionale; infatti circa il 35\% delle persone anziane vengono dimesse con nuove disabilità rispetto al livello di funzionalità di base pre-morboso. ${ }^{3}$

Gli anziani in pronto soccorso rappresentano il maggior fattore di impatto sul carico di lavoro dello staff dell'area dell'emergenza e, conseguentemente, dell'ospedale.

Nell'ambito della popolazione che ricorre al Pronto Soccorso (PS) una percentuale variabile dall'11 al 39\% riguarda soggetti ultra 65-enni; quella di età $>65$ anni si associa ad un'alta prevalenza di casi urgenti o di emergenza ( $63 \%$ vs $34 \%$ dei soggetti più giovani) e ciò sfata il luogo comune dei ricoveri degli anziani che accedono impropriamente in PS.

I pazienti di età $>75$ anni costituiscono il $12 \%$ della popolazione generale, ma ogni 100 visite effettuate in PS per anno, 38\% interessano persone di età $<65$ anni $58 \%$ riguardano soggetti di età $>75$ anni. $^{4}$

Nell'Azienda USL di Bologna la quota di accessi al PS di pazienti di età $>75$ anni è del $24 \%$ sul totale degli ultradiciottenni.

Il tasso di ospedalizzazione degli ultra 75enni è del $51 \%$ del totale e la metà di questi è costituita da frequent users $(\mathrm{FU})$ del $\mathrm{PS}$, nella classe di età più giovane soltanto il $15 \%$ dei ricoveri è riconducibile a FU. ${ }^{4}$

Le medicine Interne che garantiscono un accesso diretto e non filtrato dei pazienti dal pronto soccorso (a differenza dell'area specialistica) sono diventate la sede tipica per la degenza dei pazienti anziani e in particolare, ma certamente non solo, dove non esistono nella stessa struttura ospedaliera Unità Operative di Geriatria.

La geriatrizzazione delle strutture internistiche è 
stata vissuta inizialmente, ammettiamolo, con un certo fastidio sia per il prevalente interesse alle funzioni subspecialistiche ed alla diagnostica strumentale ma anche per il rilievo che rivestono le problematiche sociali nell'anziano ritenute generalmente non di competenza clinica. Nel tempo la flessibilità funzionale, una delle più importanti caratteristiche delle UO di Medicina Interna, ha prevalso e l'attenzione per questa popolazione è diventata significativa con il contemporaneo utilizzo, magari a volte poco conscio, di strumenti tipici dell'approccio geriatrico. Questo processo appare ancora più evidente per le Unità Operative che gestiscono anche sezioni aggregate di post-acuzie/lungodegenza.

Gli internisti devono in ogni caso riconoscere che gli specialisti geriatri hanno sviluppato negli anni passati specifiche conoscenze cliniche per: i) identificare, trattare e migliorare la prognosi delle malattie correlate all'invecchiamento (ivi compreso le fragilità, la facilità di cadute, il declino cognitivo, il delirium); ii) ridurre i rischi connessi alle ospedalizzazioni ed agli interventi chirurgici; iii) fornire le cure più appropriate ai pazienti anziani vulnerabili con comorbilità multiple e disabilità.

Queste competenze cliniche dovrebbero far parte non solo del portfolio degli specialisti geriatri, ma anche dei medici internisti e dei vari sub-specialisti di area medica.

Chi coordina e gestisce i sistemi sanitari dovrebbe essere ben consapevole di tutti i fattori in gioco che possono condizionare la sostenibilità delle cure fornite ai pazienti più anziani. ${ }^{5}$

Riguardo, poi, alla valutazione multidimensionale si tratta di un termine quasi nuovo per gli internisti italiani e che ha permesso alla Geriatria di produrre definizioni ed inquadramento nosografico della fragilità; testistica di performance per misurare e prevedere la disabilità; strumenti di seconda e terza generazione per la valutazione del bisogno clinico-assistenziale in acuzie, post acuzie, in istituzione, al domicilio; database per la valutazione di farmaco efficacia/efficienza nel vecchio escluso dai trial controllati randomizzati; trial di evidenza sulle best practices assistenziali per l'anziano ricoverato in acuzie; trial di evidenza sui servizi assistenziali migliori per il fragile.

Internisti e geriatri devono quindi collaborare per migliorare le cure dell'anziano e discutere anche di modelli assistenziali innovativi che ci aiutino a lavorare meglio su un signore o (più spesso) una signora con più di 80 anni con comorbidità $e$ in politerapia, a scelta incontinente e/o sarcopenico e/o che cade e/o osteoporotico e/o anemico e/o con problemi nutrizionali. E naturalmente che funziona male dal punto di vista fisico e/o cognitivo, che ha problemi di tono dell'umore e/o socioeconomici.

Il passo fondamentale dovrà essere il trasferimento agli internisti dell'appropriato approccio ai pazienti anziani ospedalizzati che si basa sulla comprensive geriatric assessment (CGA), una metodologia che genera un sistema di interventi e cure mediche volte a preservare l'autosufficienza e la qualità della vita.

Secondo recenti studi, l'approccio CGA ha dimostrato di ridurre la il tasso post-dimissione di istituzionalizzazione, decesso e invalidità in confronto con la gestione tradizionale, senza aumento dei costi e quindi con un peso minore sulle famiglie e sui sistemi di assistenza o di welfare. ${ }^{6,7}$

I benefici, in termini di minori tassi di disabilità e di istituzionalizzazione, sono raggiunti solo se la stessa squadra esegue sia la valutazione che la gestione clinica; viceversa, quando la valutazione è stata effettuata da un servizio con ruolo di consulenza (team) e una seconda squadra fornisce la gestione clinica, non sono stati dimostrati significativi vantaggi.

I migliori risultati si ottengono quando tutti i membri del team (medici, infermieri, assistenti sociali, membri dello staff di nutrizione e rieducazione) sono coinvolti nella gestione dei pazienti acuti anziani e hanno competenze specifiche e esperienza in geriatria

I vantaggi dell'approccio specifico alle persone anziane ricoverate basato sul CGA sono ottenuti soprattutto in specifici contesti clinici, vale a dire il cancro, insufficienza renale, e altre condizioni comuni che portano alla morte in età avanzata. ${ }^{8,9}$

Il trattamento farmacologico nei pazienti anziani ospedalizzati è un problema impegnativo sia per l'aumentato rischio di reazioni avverse al farmaco che per l'utilità limitata delle evidenze scientifiche. La mancanza di prove è dovuta all'esclusione molto comune di pazienti anziani con comorbidità da studi clinici randomizzati e meta-analisi, che generano linee guida di utilità pratica clinica per i più giovani. ${ }^{10}$

L'approccio CGA si è dimostrato efficace nel ridurre il numero di prescrizioni e le dosi giornaliere di farmaci per facilitare la sospensione dei farmaci inutili o inappropriati ma anche di ottimizzare il trattamento, aumentando il numero di farmaci assunti nei casi in cui un sotto-trattamento è stato identificato. ${ }^{11}$

La fase perioperatoria (pre-operatoria e post-operatoria) in pazienti più anziani è particolarmente delicata. In questo contesto clinico, l'approccio multidisciplinare riduce il tasso di eventi avversi. ${ }^{12}$

I pazienti anziani ricoverati in ospedale per malattie acute necessitano infine di percorsi specifici per garantire la continuità di trattamento e cura tra ospedale e domicilio; il compito specifico è quello di individuare la fragilità a tutti i livelli di cura utilizzando strumenti CGA-based idonei nelle diverse impostazioni di cure primarie. ${ }^{13}$

La collaborazione potrebbe iniziare definendo le competenze Geriatriche essenziali per l'internista in 7 aree: i) gestione dei farmaci; ii) aspetto cognitivo, affettivo e comportamentale; iii) malattia complessa o 
cronica negli anziani; iv) terapie palliative e di endlife care; v) sicurezza del paziente in ospedale; vi) transizioni di cura; vii) e gestione ambulatoriale.

Questi aspetti sono stati già peraltro oggetto anche di specifici momenti formativi promossi dalla nostra società scientifica (FADOI).

La sfida è comunque comune sia per gli internisti che per i geriatri ed è il passaggio ad una medicina problem-based orientata al paziente che prevede: ${ }^{14}$ i) promozione di un approccio globale alla valutazione clinica dei pazienti anziani con patologie multiple e politerapia; ii) valutazione della complessità del paziente e non solo la comorbidità come somma delle singole malattie; iii) impostazione delle priorità per i problemi clinici, funzionali e cognitivi; iv) identificazione di obiettivi di cura realistici, correlati all'età, agli standard di cura e alle linee guida disponibili, tenendo conto dei rischi e delle aspettative di salute del paziente; v) considerazione di comorbidità, aspettativa di vita, qualità della vita e disabilità nella valutazione clinica, e del rapporto rischio/beneficio nelle scelte diagnostiche e terapeutiche; vi) gestione del trattamento palliativo e delle cure di fine vita nei pazienti affetti da malattie incurabili; vii) considerazione delle preferenze del paziente nella pianificazione assistenziale. ${ }^{15}$

Le caratteristiche e i punti di forza della Medicina Interna sono la capacità di erogare funzioni sub-specialistiche (anche strumentali) e di diagnosi e terapia per patologie complesse o multi organo,la capacità di comunicazione e mediazione per la regia dei casi, l'elevata flessibilità funzionale, la duttilità ed economicità.

Fanno ormai parte del bagaglio dell'internista la gerarchizzazione delle priorità e la coscienza dell'interdipendenza e trasversalità nei processi assistenziali.

Va quindi fatto uno sforzo per inserire in queste caratteristiche anche l'approccio ai pazienti anziani ospedalizzati basato sulla CGA che permetterà pienamente di affermare il passaggio della Medicina Interna da medicina della complessità a medicina antropologica o della persona.

\section{Bibliografia}

1. Sanderson WC, Scherbov S. Demoghaphy. Remeasuring aging. Science 2010;329:1287-8.
2. UN Department of Economic and Social Affairs - Population Division. Conclusions. In: UN Department of Economic and Social Affairs - Population Division, ed. World population ageing 1950-2050. New York: United Nations; 2001. pp 33-34. Available from: http://www.un.org/esa/ population/publications/worldageing19502050/pdf/92cha ptervi.pdf

3. Covinsky KE, Pierluissi E, Johnston CB. Hospitalization-associated disability: "She was probably able to ambulate, but I'm not sure". JAMA 2011;306:1782-93.

4. Gardellini A, Nardi R, Arienti V, et al. Invecchiamento della popolazione, gestione dei pazienti, posti letto e sovraffollamento in ospedale. Ital J Med 2009;3:35-43.

5. Leipzig RM, Hall WJ, Fried LP. Treating our societal scotoma: the case for investing in geriatrics, our $\mathrm{Na}$ tion's future, and our patients. Ann Intern Med 2012; 156:657-8.

6. Ellis G, Whitehead MA, O'Neill D, et al. Comprehensive geriatric assessment for older adults admitted to hospital. Cochrane Database Syst Rev 2011;(7):CD006211.

7. Ellis G, Whitehead MA, Robinson D, et al. Comprehensive geriatric assessment for older adults admitted to hospital: meta-analysis of randomised trials. BMJ 2011; 343:d6553.

8. Friedrick C, Kolb G, Wedding U. Comprehensive geriatric assessment in the elderly cancer patient. Onkologie 2003;26:355-60.

9. Wiggins J, Bitzer M. Geriatric assessment for the nephrologist. Semin Dial 2012;25:623-7.

10. Cherubini A, Oristrell J, Pla X, et al. The persistent exclusion of older patients from ongoing clinical trials regarding heart failure. Arch Intern Med 2011;171:550-6.

11. Sergi G, De Rui M, Sarti S, Manzato E. Polypharmacy in the elderly: can comprehensive geriatric assessment reduce inappropriate medication use? Drugs Aging 2011; 28:509-18.

12. British Geriatrics Society. Peri-operative care for older patients undergoing surgery; January 2013. Available from: http://www.bgs.org.uk/index.php/topresources/publicationfind/goodpractice/2402-bpg-pops

13. Clegg A, Young J, Iliffe S, et al. Frailty in elderly people. Lancet 2013;381:752-62.

14. Nobili A, Garattini S, Mannucci PM. Multiple diseases and polypharmacy in the elderly: challenges for the internist of the third millennium. J Comorb. 2011;1:28-44.

15. Williams BC, Warshaw G, Fabiny AR, et al. Medicine in the 21st century: Recommended essential geriatrics competencies for Internal Medicine and Family Medicine residents. J Grad Med Educ 2010;2:373-83. 


\title{
Lean organization e learning organization a confronto per la Medicina Interna
}

\author{
Davide Croce ${ }^{1,2}$ \\ ${ }^{1}$ Centre for Research on Health Economics, Social and Health Care Management - CREMS, Carlo Cattaneo University - LIUC, \\ Castellanza (VA), Italy; ${ }^{2}$ School of Public Health, Faculty of Health Sciences, University of the Witwatersrand, Johannesburg, \\ South Africa
}

\section{RIASSUNTO}

Le organizzazioni sanitarie sono caratterizzate principalmente dalla forma organizzativa professionale, vale a dire sono organizzazioni che, al pari della scuola e della consulenza, hanno nel know how del singolo il motore della produzione, differentemente da tutte le altre organizzazioni, in particolare industriali. Riconosciuto questo assunto vengono esaminate due tipologie di approcci organizzativi: 1'organizzazione snella (la lean organization) e l'organizzazione che apprende (la learning organization). La prima deriva dai principi della produzione snella introdotti dalla Toyota negli anni ' 80 , e quindi sui processi produttivi, la seconda si basa sui principi della facilitazione della diffusione della conoscenza all'interno delle organizzazioni, e quindi sulla persona. Entrambi gli approcci hanno potenziali ricadute positive sulla Medicina interna. La lean organization sulle attività standard con la lotta agli sprechi (le attività che non generano valore per l'utente), la learning organization sulla crescita professionale (ponendo attenzione ai processi) delle persone che compongono l'organizzazione.

\section{Introduzione}

Uno dei cardini su cui si fonda l'organizzazione sanitaria, sia in ambito pubblico che in ambito privato, è la forma organizzativa professionale ed è un importante fattore di lettura organizzativa per le relazioni interne e per la progettazione. L'organizzazione professionale ${ }^{1-4}$ è caratterizzata da un'attività collettiva realizzata da personale altamente qualificato e specializzato che si avvale di conoscenze, tecniche e metodologie difficili da apprendere ed implementare, assorbite durante periodi di studio lunghi e complicati. Gli esempi di tali organizzazioni si possono individuare, ad esempio, nella scuola, nell'università, nella sanità, nella consulenza, nell'ingegneria e nella revisione contabile, cioè in tutte quelle organizzazioni dove il know how è posseduto principalmente dal singolo ed in misura minore dall'organizzazione. Infatti

Corrispondente: Davide Croce, corso Matteotti 22, 21053 Castellanza (VA), Italia.

Tel.:+39. 0331.572.424 - Fax: +39.0331.572.513.

E-mail: dcroce@liuc.it

Key words: clinical governance, learning organization, lean organization, organizzazione professionale.

Articolo pubblicato secondo la Creative Commons Attribution NonCommercial 3.0 License (CC BY-NC 3.0).

CCopyright D. Croce, 2014

Licensee PAGEPress, Italy

QUADERNI - Italian Journal of Medicine 2014; 2:83-89 l'attività è decisa ed eseguita dai singoli professionisti che compongono l'organizzazione, ai quali viene richiesta abilità e competenze che derivano dalla formazione iniziale e dallo sviluppo professionale.

I medici, ad esempio, si sentono prima di tutto appartenenti ad una specialità (ortopedici, pneumologi, internisti) e solo in seguito si percepiscono come dei professionisti appartenenti all'ospedale XY.

Il nucleo operativo è composto dai professionisti che operano con notevole autonomia (sia amministrativa che rispetto ai colleghi): limitato è il ruolo di coordinamento del management intermedio.

Per comprendere le logiche di queste organizzazioni pensiamo al ciclo di inserimento e di produzione. Se un lavoratore venisse assunto da un'impresa industriale verrebbe addestrato sui compiti del suo ruolo, attuerebbe secondo le proprie capacità, conoscenze e comportamenti (e per questo valutato) ed infine, quando lasciasse il lavoro, il know how produttivo resterebbe all'azienda, all'organizzazione e lui utilizzerebbe nella successiva azienda di impiego quanto appreso nei limiti della sovrapponibilità delle posizioni e del settore. Se invece un lavoratore professionista sanitario venisse assunto da un ospedale egli in linea di principio sarebbe addestrato sulle regole, attuerebbe secondo le proprie capacità e conoscenze su processi nei quali è (spesso) protagonista e se lasciasse il lavoro il know how resterebbe a lui e l'azienda perderebbe il proprio capitale (di conoscenza, nell'ipotesi che durante l'attività il professionista non abbia trasmesso il suo sapere ad altri). Lo stesso ciclo avverrebbe ad esempio in una società che opera nel settore dell'alta moda per il reparto gli stilisti (non per la produzione che viene gestita tradizionalmente). 
L'azione dei professionisti è influenzata da criteri e norme proprie della professione (specialità, albi, professioni, società scientifiche) e quindi definite all'esterno dell'organizzazione. Prima di tutto il professionista si sente un internista, un ortopedico o un cardiologo (per esempio), che lavora (temporaneamente) in una Azienda Sanitaria. Il confronto è sviluppato sempre in ambiti associativi ed il bravo internista viene riconosciuto dalla sua associazione (e lui cerca principalmente questa approvazione) prima che dall'azienda.

La caratteristica comune tra questa tipologia di organizzazioni, che a prima vista possono apparire del tutto differenti tra di loro se non antitetiche, è il focus su un lavoro complesso, unitamente a certe caratteristiche di stabilità del modello organizzativo. Infatti, queste organizzazioni sono basate sulla figura centrale del professionista, un lavoratore le cui capacità ed abilità lavorative sono definite attraverso uno standard di formazione e sviluppo accademico e/o professionale. ${ }^{1}$

Mintzberg $^{2}$ utilizza cinque parametri per definire l'organizzazione professionale o adhocrazia: i) organizzazione altamente organica, con poca formazione dei comportamenti; ii) specializzazione orizzontale del lavoro, basata su una formazione precedente; iii) i professionisti operano in piccoli gruppi formati a seconda della loro specializzazione per migliorare l'efficienza delle attività; iv) affidamento a meccanismi di collegamento tra diverse attività per l'aggiustamento e la coordinazione tra diversi team; v) decentralizzazione selettiva tra e dentro i team, i quali hanno diversi posizionamenti all'interno dell'organizzazione, con un mix di staff, manager e professionisti.

L'organizzazione professionale è completamente differente dalla organizzazione tradizionale, e i principi del management sono difficili da applicare: infatti, è necessario assumere e dare potere ai professionisti. Inoltre, il coordinamento stesso delle diverse unità non può essere basato sulla standardizzazione delle skills dei professionisti. ${ }^{2}$ Il livello amministrativo e quello operativo devono lavorare insieme nella stessa direzione, come un' unica forza; questo coordinamento risulta atipico per le caratteristiche elencate sopra. ${ }^{3}$

In questo contesto professionale gli svantaggi sono legati a: i) difficoltà di coordinamento tra specialisti; ii) rischi di abuso della discrezionalità inerente ai compiti professionali; iii) scarso interesse per le innovazioni.

Il nodo critico del funzionamento consiste nel bilanciamento tra 3 diversi ordini di esigenze: i) dalla qualità del servizio ai clienti; ii) dalla soddisfazione dei professionisti; iii) dal ritorno economico.

Crisi tipica porta a carenza di integrazione organizzativa: i) scarsa incisività ed efficacia degli strumenti di coordinamento e di controllo compatibili con la cultura professionale e con le condizioni di autonomia; ii) spiccato individualismo che caratterizza il funzionamento del nucleo operativo.
Il vantaggio importante di questa organizzazione è la responsabilizzazione (la presa in carico per la sanità) in una conduzione partecipata che privilegia tuttavia l'autonomia del singolo professionista.

Una organizzazione professionale presenta serie difficoltà di gestione e di indirizzo, ed i classici strumenti di management delle teorie aziendali falliscono frequentemente. Il focus deve essere posto nella motivazione che nel caso delle organizzazioni professionali è da ricercare nell'ambito delle norme della professione prima che in quelle aziendali. Sono stati introdotti da alcuni anni numerosi strumenti gestionali specifici per il settore: il loro uso combinato e coordinato costituisce la clinical governance (CG). ${ }^{5,6}$

La clinical governance ${ }^{7}$ è stata tradotta nella nostra lingua con il termine di governo clinico. La traduzione letterale adottata non corrisponde, tuttavia, in maniera corretta ai termini anglosassoni: clinical ha un significato più ampio del termine clinico italiano e andrebbe più correttamente tradotto con sanitario, mentre il termine governance ha un significato più sfumato ed esteso del termine italiano governo ed una traduzione più corretta sarebbe gestione. La clinical governance significa, secondo gli autori, coerente capacità di gestione, indirizzo e monitoraggio dei fenomeni sanitari, cioè una reale e feconda integrazione tra gestione clinico-sanitaria e gestione economico-aziendale.

Secondo Ricciardi ${ }^{8}$ una rappresentazione efficace dei concetti della clinical governance può avvenire attraverso il cosiddetto albero della governance.

La raffigurazione scelta da Ricciardi è attraente nel suo insieme in quanto mostra le reali concatenazioni e reciprocità che sussistono nelle aziende sanitarie (Figura 1).

La CG viene alimentata (si vedano le radici dell'albero) dalla gestione corretta delle risorse e dei servizi, dalla disponibilità di dati affidabili, dalla ricerca e sviluppo intimamente legata all'attività professionale ed alla cultura basata sull'apprendimento e si esplica (si vedano i rami dell'albero) attraverso l'evidence based medicine e nursing, il sistema qualità, il clinical risk management, l'audit e la valutazione, il technology assessment, la contabilità e la misurazione delle performance. A questi strumenti, che devono essere tra loro coordinati al fine di ottenere una gestione corretta delle strutture sanitarie, possiamo aggiungere la formazione continua del personale (intesa come aggiornamento e sviluppo delle capacità professionali), la funzione organizzazione nelle sue espressioni e tecniche (ad esempio lean organization, learning organization, organizzazione per dipartimenti, ecc.) ed infine una gestione aziendale animata dalla trasparenza, fondamentale elemento in una organizzazione di tipo professionale. Un ultimo grande tema del governo aziendale sanitario da aggiungere ai precedenti è quello della gestione dei team e delle equipe ed, infine, della leadership. Le ricerche 
nel settore disciplinare del management, con particolare riferimento a quello sanitario, mostrano che l'estrema complessità delle organizzazioni di servizi alla persona (probabilmente le organizzazioni maggiormente complesse tra quelle dei servizi e della produzione industriale) può essere sintetizzata solo dal leader. In altri termini, non esistono tecniche capaci di cogliere tutte le sfumature che contraddistinguono la complessa attività di organizzazione dell'assistenza sanitaria e l'uomo è l'unico in grado di cogliere gli elementi essenziali (e quindi di assumere le decisioni corrette). La differenza tra aziende sanitarie che ben funzionano in termini di risultati complessivi e di clima interno, e quelle che manifestano al contrario problematiche complesse, è spiegabile, secondo queste ricerche, per la maggior parte delle volte attraverso la gestione di un leader professionale che governa l'intera struttura.

\section{La lean organization e la medicina interna}

Il termine produzione snella (lean production) è stato utilizzato da Womack e Jones nel $1990^{9}$ illustrando il sistema di produzione che ha permesso alla Toyota di ottenere risultati di efficienza superiori alla media del settore.

Negli anni il modello della lean production è stato affinato, con altre denominazioni, quali ad esempio lean organization, lean manufacturing, lean service e lean thinking (pensiero snello), a indicarne la natura di filosofia industriale che ispira sostanzialmente tutti i metodi e le tecniche.

La lean organization (organizzazione snella nella dizione italiana) rientra in questa logica, integrando tra loro metodologie gestionali indirizzate sia alla riduzione degli sprechi che all'efficienza dei processi produttivi industriali, nel tempo trasferiti anche alla sanità. ${ }^{10}$

I principali strumenti/metodi contemplati dalla teoria lean sono: i) push vs pull: gestire processi in anticipo rispetto al fabbisogno dei clienti invece che un'azione su richiesta; ii) just in time: ottenere ciò che serve solo quando effettivamente serve; iii) Kanban: modello di reintegro delle scorte; iv) cellular manufacturing: produzione a celle di lavoratori; v) S.M.E.D.: modello per la riduzione dei tempi di set up; vi) Takt analysis: definisce il ritmo della produzione; vii) Heijunka: equilibra il carico di produzione all'interno della cella produttiva minimizzando le fluttuazioni di fornitura; viii) $5 S$ : sono i 5 passaggi fondamentali nell'approccio al miglioramento di un'area di lavoro; ix) Poka Yoke: evitare l'errore involontario.

La lean production è quindi un insieme di logiche, tecniche e strumenti per la gestione dei processi operativi, per aumentare il valore percepito dal cliente finale e ridurre sistematicamente gli sprechi. Questo è possibile solo attraverso il coinvolgimento del personale motivato al miglioramento continuo per fare sempre di più con sempre di meno (meno tempo, meno risorse strutturali, meno sforzo del personale, meno macchine, meno materiale).

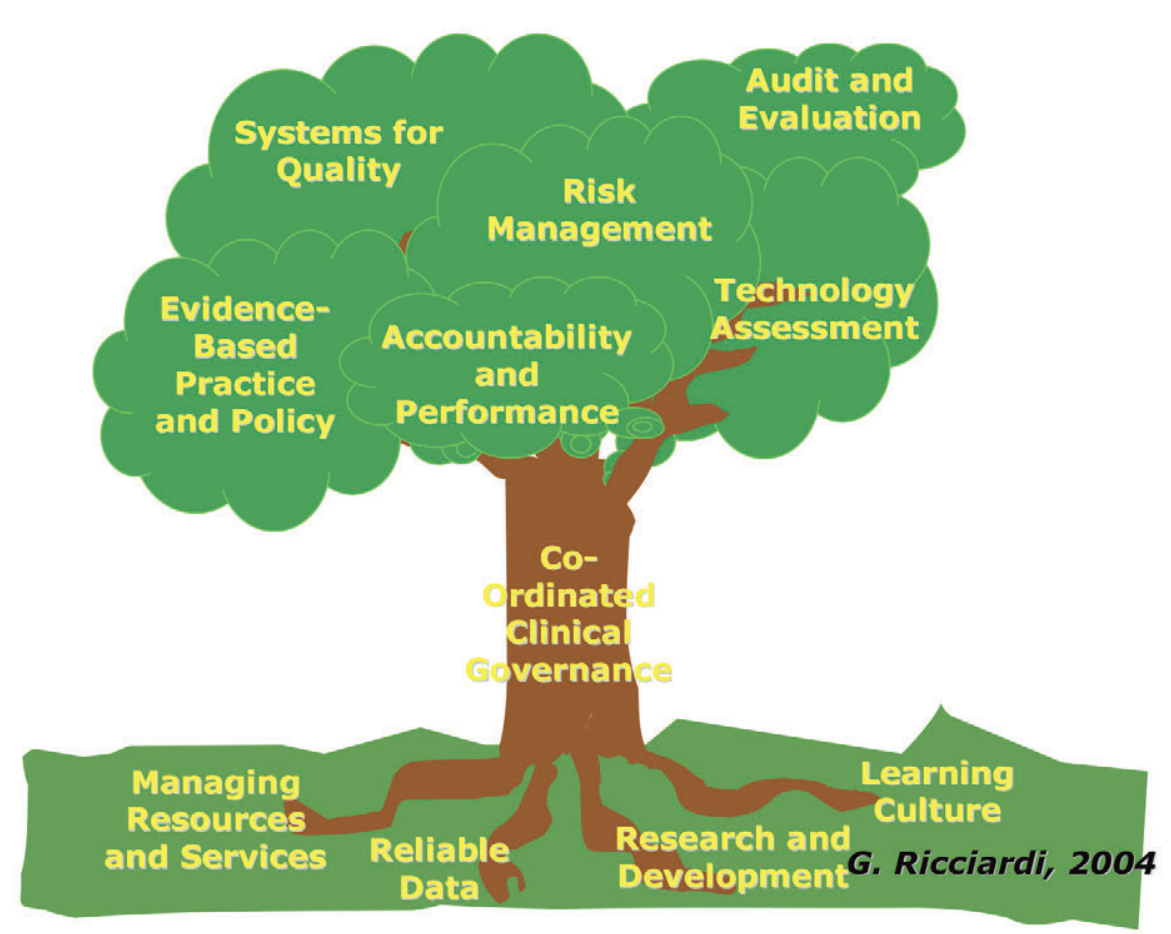

Figura 1. Clinical governance secondo Ricciardi (2004). ${ }^{8}$ 
Il concetto di lean thinking sottolinea come il lean sia innanzitutto una logica che si fonda su cinque principi: ${ }^{10}$ : i) Valore. Il punto di partenza del sistema è sempre la definizione del valore secondo la prospettiva del cliente. Valore è solo quello che il cliente è disposto a pagare; tutto il resto è spreco, e va eliminato: ii) Mappatura. Per eliminare gli sprechi occorre identificare il flusso del valore, vale a dire mappare tutte le attività che formano il processo distinguendo tra quelle con valore aggiunto e quelle non con valore aggiunto; iii) Flusso. Il processo deve scorrere in modo continuo, con tempi bassi di attraversamento (lead time) del materiale; iv) Produzione tirata. Produrre solo quello che il cliente vuole, quando e quanto lo vuole. La produzione è così tirata dal cliente (decisa su sua richiesta), anziché spinta da chi produce; v) Perfezione. La perfezione è il punto di riferimento a cui si deve tendere con un miglioramento continuo, e corrisponde alla completa eliminazione degli sprechi.

Quanto descritto ha avuto evidenze nella manifattura industriale, pur con distinguo tra culture differenti; anche nella sanità italiana sono segnalate alcune esperienze importanti (vedi Nicosia e Nicosia ${ }^{11} \mathrm{e}$ Galgano e Galgano ${ }^{12}$ tra gli autori e le aziende sanitarie di Perugia, ASF Firenze, Galliera di Genova tra le altre). In tutte questi esempi si ritrovano due elementi di comunanza: l'uso delle analogie con i modelli di assicurazione della qualità (6 Sigma in particolare) e/o la realizzazione delle esperienze nelle UUOO o servizi ove la standardizzazione dei processi/percorsi è possibile e prevalente (e.g. chirurgia/sale operatorie, laboratori di analisi, pronto soccorso).

Ma nella Medicina Interna è possibile? Con pazienti anziani e complessi?

Certamente l'approccio lean nella sua logica applicativa potrebbe portare notevoli vantaggi alle UUOO di medicina interna, con particolare riferimento alle attività standardizzate in processi (e.g. ambulatori), tuttavia non è ancora dimostrata un reale vantaggio per questa branca.

Accondiscendendo le logiche della clinical governance un'altra lettura di modello organizzativo è disponibile in letteratura, modello organizzativo che potrebbe adattarsi più facilmente alle UUOO di Medicina Interna.

\section{L'organizzazione che apprende: la learning organization}

Qualunque realtà aziendale improntata all'apprendimento organizzativo continuo quale valore dominante e costante operativa, necessita di fare della conoscenza il suo principale motore di sviluppo. Affinché ciò sia possibile, occorre governare 4 competenze chiave di apprendimento che permettono ad un'organizzazione di gestire la conoscenza e di gover- narne proficuamente i suoi flussi: ${ }^{13,14}$ i) assimilazione della conoscenza dall'esterno; ii) diffusione della conoscenza all'interno; iii) produzione di conoscenza all'interno; iv) sfruttamento della conoscenza in prodotti e servizi.

Quindi il vantaggio competitivo di un'organizzazione non trae origine tanto dall'entità della conoscenza detenuta di per sé considerata, quanto dalla capacità combinata di assimilare, diffondere, produrre e sfruttare tale conoscenza. ${ }^{14}$

Il primo passo nel perfezionare un apprendimento organizzativo consiste nella consapevolezza di tale opportunità. Sviluppare un'attività diagnostica rispetto alle reali capacità di apprendimento dell'organizzazione, oltre a costituire la base per i successivi step implementativi, rappresenta una modalità efficace per creare la comprensione e la consapevolezza ricercate. Parallelamente, una volta creata alta condivisione circa il ruolo chiave giocato dalla conoscenza, diventa essenziale comprendere anche quali siano i diversi tipi di conoscenza, nonché le modalità attraverso cui questa si conserva e si fluisce (o non si fluisce) tramite l'organizzazione. Ecco quali sono i quattro tipi di conoscenza: ${ }^{15}$ i) conoscenza del soggetto o dell'esperto su un particolare settore o una determinata disciplina; ii) conoscenza metodologica su come affrontare tipi di problemi e progetti; iii) conoscenza sociale per comprendere l'interazione umana e facilitare i processi comunicativi, soprattutto in relazione allo scambio di conoscenza; iv) know how per mettere in atto la conoscenza, basato sulla pratica e l'esperienza.

Al tempo stesso, la conoscenza viene conservata secondo quattro possibili modi: ${ }^{16}$ i) nella mente degli individui, dall'istruzione scolastica, all'esperienza, allo scambio di informazioni e così via; ii) nei sistemi tecnici, grazie alle attività di raccolta, analisi, codifica, ristrutturazione e riprogettazione di dati, come, ad esempio, i manuali, le procedure lavorative e le struttura di database; iii) nei sistemi di management, a prescindere dal loro livello di formalità, quali regole per le riunioni, stili di management, gestione dei progetti, controllo di qualità e gestione delle informazioni; iv) nelle norme e nei valori, quale la comprensione condivisa di come dovrebbe avvenire l'apprendimento, come pure la lealtà, la curiosità e le modalità per affrontare i conflitti di interesse.

Il secondo passo di questo modello consiste nella diagnosi delle capacità di apprendimento dell'organizzazione, che dovrà essere accompagnata da quattro scale di bilanciamento relative alle quattro competenze chiave in cui si articola il medesimo (Tabella 1). Fondamentalmente, questa attività dovrebbe permettere di dare risposta a tre domande cruciali in materia di apprendimento organizzativo, ovvero quali strumenti sono disponibili per l'apprendimento (e quali vengono realmente utilizzati), qual è l'azione svolta dal management 
per dirigere l'apprendimento e, in ultimo, qual è il livello di bilanciamento tra la conoscenza detenuta dagli individui e quella intrinseca ai sistemi.

Il terzo step implica la necessità di dare risposta alle criticità emerse nella precedente fase diagnostica mediante opportuni interventi correttivi. Per questo processo esistono cinque fasi: ${ }^{13}$ i) analisi e discussione dei problemi e degli ostacoli riscontrati; ii) approfondimento delle cause dei problemi tra i membri dell'organizzazione in fasi discendenti di astrazione; iii) formulazione conseguente di obiettivi di apprendimento e policy di gestione della conoscenza; iv) focus sulle soluzioni per superare gli ostacoli all'apprendimento; v) implementazione e sviluppo dei necessari strumenti di apprendimento.

L'elemento che caratterizza positivamente il modello si legge nella dimensione concettuale assecondata in materia di apprendimento organizzativo, ritenendo che lo stesso debba essere sviluppato mediante l'identificazione e la sollecitazione di opportune competenze, e non banalmente tramite la creazione di un progetto o la riprogettazione dell'organizzazione in un'immagine.

\section{Discussione e Conclusioni}

Entrambe le teorie organizzative presentate nei precedenti paragrafi mostrano analogie ed affinità con l'organizzazione professionale; inoltre presenterebbero vantaggi nell'applicazione alle UUOO di Medicina interna, anche se difficilmente un approccio organizzativo funziona se isolato, ma solo se coerente con i meccanismi operativi di confine. ${ }^{1}$

L'organizzazione snella, derivante dalla produzione snella della Toyota, ha elementi allettanti nel- l'approccio. Tagliare gli sprechi, programmare l'attività, rivedere i processi sono solo alcuni degli elementi che potrebbero aiutare la medicina interna a migliorare l'efficienza (e perché no anche a volte l'efficacia dell'azione) purché abbinata alla clinical governance (ambiente di eccellenza e qualità delle prestazioni per gli utenti).

L'organizzazione che apprende è meno appeal nella sua teoria, meno alla moda, ma probabilmente più adeguata alla logica delle organizzazioni professionali. Progettare l'apprendimento dei collaboratori non è agevole ma potrebbe apparire alla lunga altrettanto sfidante che ridurre gli sprechi.

Un altro elemento da prendere in considerazione sulla comparazione tra i due approcci teorici è il fattore umano. L'approccio alla progettazione organizzativa non può prescindere dal fattore umano disponibile, ed entrambi gli approcci richiedono un forte coinvolgimento anche emotivo del personale.

La Tabella 2 illustra la comparazione tra i modelli e le implicazioni per la medicina interna.

Come indicazione dalla tabella possiamo affermare che entrambi i modelli proposti hanno una validità per la Medicina interna e si possono prendere in esame per la sua introduzione alcuni degli strumenti operativi di entrambi i modelli, Per esempio in una prima fase risulterà più semplice l'introduzione della learning organization secondo i principi della conoscenza, operando in un secondo tempo la lotta agli sprechi dopo aver sistematizzato i principali progressi secondo le evidenze di letteratura e di conoscenza del gruppo.

L'analisi proposta ha alcuni evidenti limiti intrinseci. È teorica, ex ante, e non ha misurazioni di confronto sugli outcome tra modelli come un trial controllato randomizzato. È inoltre basata sulla sensibilità organizzativa dell'autore.

Tabella 1. Strumenti per la crescita delle competenze di apprendimento organizzativo.

\begin{tabular}{llll}
\hline Assimilazione & Diffusione & Produzione & Sfruttamento \\
\hline Reti esterne professionali & Assemblaggio e utilizzo di manuali & Gestione di programmi e progetti & $\begin{array}{l}\text { Team di progetto } \\
\text { interdisciplinari }\end{array}$ \\
\hline Istruzione & Regolamenti e procedure & Concetti di report e prodotti finali & Ricerca di mercato \\
\hline Conferenze & Sistemi informativi di conoscenza & Riesame della qualità & $\begin{array}{l}\text { Promozione della conoscenza } \\
\text { (internamente ed esternamente) }\end{array}$ \\
\hline Contatti con gli utenti & Studio della best practice & Apprendimento delle azioni & $\begin{array}{l}\text { Miglioramento dei prodotti in } \\
\text { base alle critiche dei clienti }\end{array}$ \\
\hline Analisi cooperativa & Scambio interno di conoscenze & Dialogo & \\
\hline Cooperazione dei fornitori & Addestramento e guida & Autovalutazione & \\
\hline Ricerca & Valutazione dai propri pari & $\begin{array}{l}\text { Valutazione delle performance } \\
\text { e ricompense }\end{array}$ & \\
\hline Gestione della conoscenza & Reti informali & $\begin{array}{l}\text { Riprogettazione del processo } \\
\text { aziendale }\end{array}$ & \\
\hline Scenari creativi & Rotazione delle mansioni & Feedback professionale & \\
\hline
\end{tabular}


Riconosciamo infine che i due approcci si riconducono alla classica dualità di visione delle organizzazioni. Le teorie dell'organizzazione hanno infatti mostrato come, in una prospettiva storica e multidisciplinare, si siano sviluppate molteplici e concorrenti modalità di concepire l'organizzazione,,${ }^{17} \mathrm{ma}$ due sono i modelli principali ispiratori del concetto di organizzazione ancora oggi: la metafora dell'organizzazione meccanica (come macchina) e quella dell'organizzazione organica (come organismo vivente).

L'organizzazione come macchina, primo supporto come disciplina all'efficienza delle imprese, nasce come conseguimento dei vantaggi ottenuti dalla divisione del lavoro. Taylor e la sua teoria dell'organizzazione scientifica del lavoro (primi del '900) fu il principale artefice della ricerca di massimizzazione dell'efficienza produttiva attraverso la leva di divisione del lavoro, spinta a livelli molto accentuati sulla base di studi analitici delle operazioni elementari effettuate dai lavoratori. Questa standardizzazione assunse il massimo splendore nell'industria automobilistica con Henry Ford che la usò come leva espansionistica nel nuovo mercato del trasporto. I tempi e metodi vengono utilizzati nel taylorismo come base per la progettazione del lavoro, e gli operatori devono conformarsi il più possibile alle istruzioni ricevute. Si riconduce quindi l'impiego del fattore umano ad una sequenza di attività programmate in modo da massimizzarne il rendimento. Questa visione organizzativa, tendenzialmente superata, è talvolta ancora oggi fonte di ispirazione (la burocrazia, Weber), addirittura comoda come un vestito per i lavoratori in quanto rassicurante (ma al contempo alienante). Pensiamo in questo caso all'organizzazione militare. La concezione di organizzazione come macchina, cioè con un funzionamento dominabile attraverso la regolazione formale, può essere equo e affidabile per il funzionamento delle istitu- zioni, meno per le imprese. Per raggiungere questo la condizione meccanica occorre definire confini netti e ben presidiati dell'organizzazione rispetto alla realtà esterna ed alle componenti verticali (gerarchia) ed orizzontali (funzioni e compiti ben delineati). L'organizzazione ricerca condizioni di stabilità, sicurezza e ripetitività come fattori fondamentali per l'efficienza dei processi produttivi, ${ }^{18}$ mentre la spersonalizzazione con l'oggettività, neutralità e trasparenza sono i fini della burocrazia. ${ }^{1}$

La visione dell'organizzazione che si è opposta negli anni al modello meccanico dopo diversi filoni di ricerca (motivazione, socio-tecnico, sistema aperto, ma soprattutto human relations) è quello dell'organismo vivente, peraltro reso attuale dalla new economy e dalle organizzazioni di servizi o di ricerca. Le parole chiave sono arricchimento delle posizioni come motivazione al lavoro, stile di direzione partecipativo e coinvolgimento degli operatori. Lo scambio con l'ambiente esterno è continuo e come gli organismi viventi l'organizzazione deve essere aperta alla relazione nel suo ambiente di riferimento per poter stabilire un rapporto adeguato alla sua sopravvivenza nel lungo periodo. ${ }^{1}$ L'assetto organizzativo organico è funzionale in un ambiente in cambiamento, così come il meccanico lo è in un ambiente stabile. L'organizzazione organica è tendenzialmente auspicata come modello da quasi tutti gli operatori sanitari, tuttavia è un modello che richiede molte energie e quindi faticoso da mantenere, al contrario della rassicurazione e stabilità del modello meccanico (Tabella 3).

La conclusione ovvia è che le due metafore possono convivere in aree differenti nella stessa organizzazione (pensiamo ai laboratori analisi - lean organization ed alla Medicina Interna - learning organization) e così anche lo stile di direzione, e che il modello di riferimento per l'organizzazione dipende anche dal ciclo di vita dell'organizzazione stessa.

Tabella 2. Comparazione tra i due modelli organizzativi.

\begin{tabular}{llll}
\hline & Lean organization & Learning organization & Implicazioni per la medicina interna \\
\hline Focus del modello & Processi & Personale & - \\
\hline Strumenti operativi & $\begin{array}{l}\text { Revisione processi, eliminare le } \\
\text { attività non a valore aggiunto, } \\
\text { controllo degli armadi di reparto, } \\
\text { tempi di produzione, controllo } \\
\text { degli errori }\end{array}$ & $\begin{array}{l}\text { Assimilazione della conoscenza } \\
\text { dall'esterno, diffusione della } \\
\text { conoscenza all'interno, } \\
\text { produzione di conoscenza } \\
\text { all'interno, sfruttamento della } \\
\text { conoscenza in prodotti e servizi }\end{array}$ & $\begin{array}{l}\text { In una prima fase meno traumatico } \\
\text { il modello di apprendimento, } \\
\text { i due modelli potrebbero essere } \\
\text { usati in contemporanea }\end{array}$ \\
\hline $\begin{array}{llll}\text { Tempi di introduzione } \\
\text { Rapido in una prima fase, medio } \\
\text { periodo per l'assimilazione della } \\
\text { mentalità di revisione continua }\end{array}$ & $\begin{array}{l}\text { Lento, risultati nel medio periodo } \\
\text { dopo investimento sul personale }\end{array}$ & Dipende dal ciclo di vita del reparto \\
\hline $\begin{array}{l}\text { Schema organizzativo } \\
\text { di fondo }\end{array}$ & $\begin{array}{l}\text { Meccanico e poi organico sul } \\
\text { personale }\end{array}$ & $\begin{array}{l}\text { Organico e poi meccanico con la } \\
\text { strutturazione della conoscenza }\end{array}$ & -
\end{tabular}


Tabella 3. Confronto tra le caratteristiche del modello meccanico e organico.

\begin{tabular}{ll}
\hline Modello meccanico & Modello organico \\
\hline Netta separazione tra unità di compiti e funzioni & Compiti assegnati in funzione delle necessità del periodo \\
\hline Formalizzazione precisa di compiti e responsabilità & Responsabilità diffusa e adattamento dei compiti in funzione delle necessità organizzative \\
\hline Gerarchia come perno organizzativo & Gerarchia come informazione e competenze \\
\hline Relazioni essenzialmente verticali & Relazioni orizzontali come necessità per la realizzazione dei compiti \\
\hline Stile di direzione verticale & Stile di direzione partecipativo \\
\hline Status derivante dalla posizione gerarchica & Status derivante dalla competenza professionale e dalle capacità contributive \\
\hline
\end{tabular}

\section{Bibliografia}

1. Rebora G. Manuale di organizzazione aziendale. Roma: Carocci Ed.; 2001.

2. Mintzberg H. Structure in fives: designing effective organizations. London: Prentice-Hall International; 1983.

3. Mintzberg H. Mintzberg on management. London: Collier Macmillan Publ.; 1989.

4. Mintzberg H. La progettazione dell'organizzazione aziendale. Bologna: Il Mulino; 1996.

5. Wright J, Hill P. La governance clinica (edizione italiana a cura di Favaretti C.). Milano: McGraw-Hill; 2005.

6. Grilli R, Taroni F. Governo clinico. Roma: Il pensiero scientifico editore; 2004.

7. Scally G, Donaldson L. Clinical governance and the drive for quality improvement in the new NHS in England. BMJ 1998;317:61.

8. Ricciardi W. Relazione introduttiva al convegno "Clinical Governance: una prospettiva organizzativa e gestionale", 30 aprile 2004, Scuola di Management Luiss Guido Carli, Roma.

9. Womack J, Jones D, Roos D. The machine that changed the World: the story of lean production. New York: Harper Collins; 1990.

10. Shah R, Ward PT. Defining and developing measures of lean production. J Operat Manage 2007;25:785-805.

11. Nicosia PG, Nicosia F. Tecniche lean in sanità. Più valore, meno spreco, meno errori col sistema Toyota. Milano: Franco Angeli Ed.; 2008.

12. Galgano A, Galgano C. Il sistema Toyota per la sanità. Milano: Guerini Next; 2006.

13. Sprenger $\mathrm{C}$, ten Have S. Kennismanagement alsmoter van de lerende organisatie. Holland Manage Rev 2006;Sep-Oct;73.

14. AA.VV. L'organizzazione che apprende. Sanità Pubblica e Privata 2007; 1 .

15. Berenschot M. Modelli di management. Idee e strumenti. Milano: Pearson Education; 2005.

16. Quinn JB. Intelligent enterprise. Boston: Free Press; 1992.

17. Morgan G. Images: Le metafore dell'organizzazione. Milano: Franco Angeli; 1991.

18. Gaj A. Mercati interni del lavoro e sull'organizzazione. Manuale per i corsi dell’Università di Bergamo; 2007. 


\title{
Clinical governance: ultima spiaggia per salvare la sanità pubblica?
}

\author{
Antonino Cartabellotta \\ Fondazione GIMBE, Bologna, Italia
}

\section{RIASSUNTO}

Un servizio sanitario pubblico, equo e universalistico rappresenta una conquista sociale irrinunciabile per l'eguaglianza di tutti i cittadini: mettere in discussione la sanità pubblica significa compromettere non solo la salute, ma soprattutto la dignità dei cittadini e la loro capacità di realizzare ambizioni e obiettivi che, in ultima analisi, dovrebbero essere viste dalla politica come il vero ritorno degli investimenti in sanità.

\section{Introduzione}

Da quando la sommatoria di varie manovre finanziarie, tutte avvenute al di fuori dell'accordo Stato-Regioni, ha sottratto alla sanità pubblica una cifra stimata tra 25 (dallo Stato) e 30 (dalle Regioni) miliardi di euro, il tema della sostenibilità del Servizio Sanitario Nazionale (SSN) è balzato all'onore delle cronache. In particolare, alla fine del 2012 la Conferenza delle Regioni e delle Province Autonome ha paventato che a partire dal 2014 i tagli rischiano di portare al collasso il SSN, mettendo a rischio la possibilità di garantire tutte le prestazioni sanitarie e socio-sanitarie oggi erogate sul territorio nazionale. A fronte di questa affermazione, se è legittimo chiedersi quanto la deriva economicista della politica possa erodere il diritto costituzionale alla salute, è altrettanto indispensabile ribadire che l'articolo 32 della Costituzione tutela il diritto alla salute e non alla sanità, oggi intesa come disponibilità illimitata $\mathrm{e}$ tempestiva di servizi e prestazioni sanitarie, incluse quelle inefficaci e inappropriate che aumentano i rischi per i pazienti consumando preziose risorse.

Anche se posta al centro della scena solo in tempi recenti, la sostenibilità del SSN da almeno 20 anni è soggetta a una silenziosa erosione conseguente a vari

Corrispondente: Antonino Cartabellotta, Fondazione GIMBE, via Amendola 2, 40121 Bologna, Italia.

Tel.: +39.051.5883920 - Fax: +39.051.3372195.

E-mail: nino.cartabellotta@gimbe.org

Parole chiave: Servizio Sanitario Nazionale, sostenibilità, politiche sanitarie, sprechi, clinical governance, appropriatezza.

Articolo pubblicato secondo la Creative Commons Attribution NonCommercial 3.0 License (CC BY-NC 3.0).

(C) Copyright A. Cartabellotta, 2014

Licensee PAGEPress, Italy

QUADERNI - Italian Journal of Medicine 2014; 2:90-94 fattori: le mutate condizioni demografiche, economiche e sociali, la crescente introduzione sul mercato di false innovazioni tecnologiche, le conseguenze della modifica del Titolo V della Costituzione, il perpetuarsi delle ingerenze della politica partitica nella programmazione sanitaria, la grande incompiuta dei LEA, la gestione delle aziende sanitarie come silos in continua competizione, l'evoluzione del rapporto paziente-medico, l'involuzione del cittadino in consumatore di servizi e prestazioni sanitarie. A fronte di questo progressivo indebolirsi della sostenibilità del SSN, la politica sanitaria si è limitata a occasionali rattoppi: infatti, dopo il D. Lgs. 229/99, solo interventi legislativi dettati dalla contingenza e dall'urgenza e non certo frutto di una programmazione coerente con i bisogni socio-sanitari della popolazione.

Oggi, in un contesto particolarmente critico, teatro di un continuo confitto istituzionale tra Stato e Regioni, è indispensabile coinvolgere tutti gli stakeholders del pianeta salute per fornire risposte di sistema a cinque questioni chiave da cui dipende il futuro del nostro SSN. ${ }^{1}$

\section{Il Servizio Sanitario Nazionale è realmente sotto-finanziato?}

I dati dell'OCSE indicano che in Italia la spesa sanitaria - da tempo inferiore a quella dei più importanti paesi europei - continua a scendere, ${ }^{2}$ segnando nel 2012 un record negativo del-3\%. L'Italia, dove 1' $80 \%$ della spesa sanitaria è pubblica, è tra i paesi che spendono meno sia in termini di incidenza sul PIL, sia in termini di spesa pro-capite. Infatti, a parità di potere d'acquisto, ogni cittadino italiano dispone per la propria salute di 3027 dollari l'anno, rispetto agli 8388 degli americani, ai quasi 6000 di norvegesi, svizzeri e olandesi, ai 4500 di austriaci, tedeschi e danesi o ai 4100 dei francesi. Di fatto, l'Italia è fanalino di coda insieme a Spagna, Portogallo, Grecia e tutti i paesi dell'est Europa. Tuttavia, a fronte di questi incontrover- 
tibili dati economici, è bene ricordare che nei sistemi sanitari avanzati non esistono evidenze scientifiche che sostengono una correlazione positiva tra entità degli investimenti in sanità e miglioramento degli esiti di salute delle popolazioni. ${ }^{3}$

Il Patto per la Salute, approvato dal Ministero della Salute e dalle Regioni, ${ }^{4}$ sembra aver messo in sicurezza il finanziamento del SSN, fissando le risorse destinate alla sanità pubblica sino al 2016. Tuttavia, il Ministero dell'Economia e delle Finanze non ha rinunciato alla precisazione salvo eventuali modifiche che si rendessero necessarie in relazione al conseguimento degli obiettivi di finanza pubblica e a variazioni del quadro macroeconomico. In altre parole, nonostante l'impegno e le continue rassicurazioni del Ministro Lorenzin, il Patto per la Salute non scongiura affatto ulteriori tagli per esigenze di finanza pubblica.

\section{Il modello di politica sanitaria che ha generato 21 sistemi regionali deve essere ripensato?}

A fronte di un diritto costituzionale che garantisce universalità ed equità di accesso a tutte le persone $\mathrm{e}$ alla L. 833/78 che conferma la globalità di copertura in base alle necessità assistenziali dei cittadini, dati e fatti smentiscono continuamente i princìi fondamentali su cui si basa il SSN. ${ }^{5}$ Infatti, le inaccettabili diseguaglianze regionali documentano che l'universalità e l'equità di accesso ai servizi sanitari, la globalità di copertura in base alle necessità assistenziali dei cittadini, la portabilità dei diritti in tutto il territorio nazionale e la reciprocità di assistenza tra le Regioni rappresentano oggi un lontano miraggio. Inoltre, la stessa attuazione dei princìpi organizzativi del SSN è parziale e spesso contraddittoria: infatti, la centralità della persona, la responsabilità pubblica per la tutela del diritto alla salute, la collaborazione tra i livelli di governo del SSN, la valorizzazione della professionalità degli operatori sanitari e l'integrazione socio-sanitaria presentano innumerevoli criticità. Considerato che la recente controriforma del Titolo $\mathrm{V}$ ha eliminato la legislazione concorrente e confermato l'autonomia organizzativa delle Regioni, è indispensabile ampliare le capacità di indirizzo e verifica del Ministero della Salute sui 21 sistemi sanitari regionali, in particolare attraverso la definizione di standard clinico-assistenziali e indicatori di performance unitari per tutto il territorio nazionale.

\section{La modalità di organizzazione e gestione delle aziende sanitarie è adeguata?}

Pianificazione, organizzazione ed erogazione dell'assistenza sanitaria incontrano numerosi ostacoli, legati in parte all'eterogeneità delle aziende sanitarie, in parte a modalità gestionali poco compatibili con il prodotto salute. Innanzitutto, l'approccio al management continua a essere guidato dalla produttività (output-driven) e non dagli esiti di salute (outcome-driven): di conseguenza, la valutazione e il finanziamento delle aziende sanitarie sono basati quasi esclusivamente sul binomio produttività-consumi. In secondo luogo, le differenti modalità di finanziamento tra aziende sanitarie che erogano gli stessi servizi generano interessi in competizione. Infine, esistono notevoli resistenze e difficoltà ad attuare modalità sovra-aziendali di organizzazione dell'assistenza: modelli per intensità di cura, hub-spoke, transitional care, etc.

\section{I professionisti sanitari possono contribuire alla sostenibilità del Servizio Sanitario Nazionale?}

Secondo il principio di giustizia distributiva se l'etica del razionamento appartiene alla politica, quella della riduzione degli sprechi è legata alla professionalità dei medici, con le loro prescrizioni diagnosticoterapeutiche. ${ }^{6}$ Di conseguenza, guidati da un equilibrato mix di evidenze e buonsenso, tutti i professionisti sanitari - medici in particolare - dovrebbero collaborare con le Istituzioni per identificare gli interventi sanitari inefficaci, inappropriati e dal low value, che riducono l'efficacia dell'assistenza, aumentano il rischio clinico per i pazienti e determinano un ingente spreco di risorse. ${ }^{7-10}$ Inoltre, quando le evidenze scientifiche non supportano le richieste del paziente, il medico ha sempre il dovere di rifiutarle, convincendolo attraverso una evidence-based persuasion, ${ }^{11}$ e contribuendo a riformulare l'imperativo socio-culturale dominante more is better - che ha trasformato il cittadino/paziente in consumatore - in less is more, vessillo di una medicina parsimoniosa. ${ }^{12}$

\section{Quali responsabilità per cittadini e pazienti?}

Il SSN è a disposizione delle persone che di volta in volta assumono ruoli differenti: sono pazienti, quando presi in carico per problemi assistenziali, utenti quando si rivolgono per informazioni, transazioni, certificazioni, cittadini quando con il loro voto contribuiscono alle scelte politiche. ${ }^{13}$ Oggi la progressiva involuzione del cittadino in consumatore di servizi e prestazioni sanitarie fa il gioco della politica, perché la customer satisfation rappresenta un insostituibile strumento di consenso elettorale. ${ }^{14}$ Peraltro, solo pochi contribuenti sono consapevoli che la domanda inappropriata di servizi e prestazioni sanitarie concorre agli sprechi del SSN, con pesanti ricadute sotto forma di imposte locali e di mancate detrazioni, in particolare nelle regioni in piano di rientro. 
In un simile contesto, l'obiettivo del SSN finisce inevitabilmente per identificarsi con l'erogazione di servizi e prestazioni sanitarie, un'attività influenzata sia da logiche di profitto, sia da conflitti di interesse a vari livelli, a dispetto di quanto sancito dalla L. 833/78 che istituiva il SSN per promuovere, mantenere, e recuperare la salute fisica e psichica di tutta la popolazione. Oggi chi spalleggia la non sostenibilità del SSN è consapevole che il mercato della sanità è regolato dall'offerta di servizi e prestazioni sanitarie in grado, al tempo stesso, di modulare e soddisfare la domanda dei cittadini: da un lato inducendo i consumi (professionisti, aziende sanitarie), dall'altro attuando il razionamento implicito (politica sanitaria). Queste regole sopravvivono indisturbate, sia perché il finanziamento di servizi e prestazioni sanitarie si basa su criteri quantitativi che non tengono conto della loro efficacia e appropriatezza, sia perché la soddisfazione dei cittadini costituisce un potente strumento di consenso elettorale.

Oggi che la sostenibilità irrompe prepotentemente sulla scena, l'equilibrio offerta-domanda rischia di saltare perché l'involuzione del SSN ha generato sia una ipertrofia di strutture e servizi, sia un livello di inappropriatezza di prestazioni che il finanziamento pubblico non è più in grado di mantenere. Questo circolo vizioso può essere spezzato solo attuando politiche sanitarie coerenti con una delle seguenti strategie:

- Aumentare il finanziamento del Servizio Sanitario Nazionale: nella realistica impossibilità di disporre di ulteriori risorse pubbliche, questo può essere ottenuto attraverso l'intermediazione finanziaria e assicurativa dei privati, che aumenta le diseguaglianze sociali, e/o mediante aumento della compartecipazione alla spesa da parte dei cittadini, una misura troppo impopolare per la politica oltre che fortemente condizionata dalla crisi economica. In ogni caso, senza adeguate strategie per prevenire inefficienze e sprechi, buona parte delle risorse aggiuntive finirebbe paradossalmente per finanziare servizi e prestazioni inutili, determinando ulteriori sprechi.

- Effettuare tagli lineari: è la strada percorsa sinora dalla politica: riduzione dei posti letto, blocco delle assunzioni, riduzione delle tariffe di rimborso per le prestazioni ospedaliere e ambulatoriali, soppressione di servizi. Strategia semplice da attuare, ma per la sua scarsa selettività rischia di privare un numero sempre più elevato di cittadini di servizi essenziali e appropriati.

- Eliminare gli sprechi: attraverso il coinvolgimento di tutte le categorie di stakeholders, questa strategia prevede l'identificazione e la riduzione degli sprechi che aumentano i costi dell'assistenza, senza produrre alcun beneficio. ${ }^{15}$ In particolare, secondo la tassonomia di Don Berwick, 6 categorie di sprechi assorbono circa il $20 \%$ della spesa sanitaria (Tabella 1), che in Italia equivale a una cifra superiore ai 20 miliardi di euro. Al di là delle dichiarazioni di intenti e di quanto stabilito dal Patto per la Salute (art. 1, comma 4: I risparmi derivanti dall'applicazione delle misure contenute nel Patto rimangono nella disponibilità delle singole regioni per finalità sanitarie), questo obiettivo è realisticamente perseguibile solo se tutti gli attori della sanità, accantonando definitivamente ogni interesse individuale e di categoria, sono disponibili a riallinearsi sul vero obiettivo del SSN. Infatti, se è indiscutibile il principio che tutte le risorse recuperate devono rimanere nel comparto sanitario, in assenza di chiari obiettivi di disinvestimento e riallocazione, la maggior parte delle regioni non riuscirà mai nella titanica duplice impresa di tagliare gli sprechi e investire su servizi e prestazioni sottoutilizzate, oltre che effettuare i necessari investimenti strutturali.

Per intervenire in maniera efficace su alcune categorie di sprechi - in particolare il sovra-utilizzo di interventi sanitari inefficaci e inappropriati (overuse $^{16}$ ), il sotto-utilizzo di interventi sanitari efficaci e appropriati (underuse) e l'inadeguato coordinamento dell'assistenza tra ospedale e cure primarie - è indubbio che gli strumenti della clinical governance rappresentano da tempo un riferi-

Tabella 1. Categorie di sprechi e relativo impatto sul Sistema Sanitario Nazionale.

\begin{tabular}{|c|c|c|}
\hline Categoria sprechi & \% sprechi & Mld di $€$ \\
\hline 1. Sovra-utilizzo di interventi sanitari inefficaci e inappropriati & 26 & 5,72 \\
\hline 2. Frodi e abusi & 21 & 4,62 \\
\hline 3. Tecnologie sanitarie acquistate a costi eccessivi & 19 & 4,18 \\
\hline 4. Sottoutilizzo di interventi sanitari efficaci e appropriati & 12 & 2,64 \\
\hline 5. Complessità amministrative & 12 & 2,64 \\
\hline 6. Inadeguato coordinamento dell'assistenza & 10 & 2,20 \\
\hline
\end{tabular}

Tassonomia e percentuali sprechi da Bervick et al. ${ }^{15}$. 
mento assoluto, indispensabile per migliorare sia l'appropriatezza professionale, sia quella organizzativa. Tuttavia, questa strategia di politica sanitaria, in Italia non ha mai inciso realmente sulla programmazione sanitaria nazionale e regionale, né ha condizionato in maniera significativa le pratiche professionali, nonostante l'impegno attivo di alcune società scientifiche. ${ }^{17}$

A sorpresa, il Patto per la Salute 2014-2016, disegnando le politiche sanitarie del prossimo triennio, relativamente all'assistenza ospedaliera (art. 3) conviene sull'adozione del regolamento sugli standard qualitativi, strutturali, tecnologici e quantitativi, in attuazione del decreto Balduzzi. In altre parole, quella bozza che per oltre 18 mesi è rimasta parcheggiata in attesa di essere riesumata o gettata definitivamente alle ortiche viene inserita nel Patto per la Salute e approvata dalla Conferenza delle Regioni e delle Province Autonome il 5 agosto. ${ }^{18}$ Senza entrare nel merito degli aspetti relativi alla riorganizzazione dei posti letto, è indubbiamente degno di nota che la clinical governance, sopravvissuta ai ripetuti tentativi di archiviazione, torna ad essere a pieno titolo lo strumento di riferimento per garantire qualità e sostenibilità dell'assistenza sanitaria, almeno in ambito ospedaliero. Nell'incipit dell'Allegato 1 si legge infatti che $i$ significativi cambiamenti registrati in questi anni in tema di assistenza sanitaria ed in particolare di quella ospedaliera richiedono un sostanziale ammodernamento del SSN, partendo da alcune tematiche prioritarie, come l'implementazione della Clinical Governance [...].

Ma è nella sezione 5 (standard generali di qualità), che si concretizza il vero e proprio rinascimento del governo clinico: si legge, infatti, che è necessario promuovere ed attivare standard organizzativi secondo il modello di Clinical Governance, per dare attuazione al cambiamento complessivo del sistema sanitario e fornire strumenti per lo sviluppo delle capacità organizzative necessarie a erogare un servizio di assistenza di qualità, sostenibile, responsabile (accountability), centrato sui bisogni della persona e che le strutture ospedaliere declinano le dimensioni della clinical governance, secondo linee di indirizzo e profili organizzativi. In particolare, gli standard organizzativi per tutti i presidi ospedalieri (di base, di I e II livello) sono costituiti dalla documentata e formalizzata presenza di sistemi o attività di: i) gestione del rischio clinico: dai sistemi di segnalazione di eventi avversi alle procedure di identificazione del paziente; dalla checklist operatoria alla scheda unica di terapia; dai sistemi di reporting delle infezioni alla sorveglianza microbiologica; dai protocolli per la profilassi antibiotica a quelli per il lavaggio delle mani; ii) evidence-based medicine: dalla definizione all'implementazione di percorsi assistenziali secondo linee guida esistenti; iii) health technology assessment (HTA): dall'utilizzo dei report HTA nazionali e interna- zionali alla partecipazione alle reti HTA coordinate dall'Age.Na.S.; iv) valutazione e miglioramento continuo delle attività cliniche: dell'audit clinico alla misurazione della performance clinica, degli esiti e della qualità percepita; v) documentazione sanitaria, comunicazione, informazione e partecipazione del cittadino/paziente: dall'integrazione dei sistemi informativi alla trasmissione di informazioni gestionali e sanitarie; dall'informazione ai cittadini/pazienti al loro coinvolgimento attivo nel processo di cura; vi) formazione continua del personale: dalla rilevazione sistematica di fabbisogni formativi alla valutazione dell'efficacia dei programmi di formazione continua attuati.

Questo elenco non rappresenta affatto una novità: infatti era già stato proposto nel Piano Sanitario Nazionale 2011-2013, ${ }^{19}$ che aveva sapientemente introdotto l'approccio di sistema alla clinical governance realizzata tramite l'integrazione di numerosi fattori tra di loro interconnessi e complementari, tra i quali vi sono la formazione continua, la gestione del rischio clinico, l'audit, la medicina basata sulle evidenze, le linee guida cliniche e i percorsi assistenziali, la gestione dei reclami e dei contenziosi, la comunicazione e gestione della documentazione, la ricerca e lo sviluppo, la valutazione degli esiti, la collaborazione multidisciplinare, il coinvolgimento dei pazienti, l'informazione corretta e trasparente e la gestione del personale. Anche il Sistema di Formazione Continua in Medicina ${ }^{20}$ annovera tutti gli strumenti della clinical governance tra gli obiettivi di processo e di sistema due dei tre pilastri che concorrono alla costruzione del dossier formativo di tutti i professionisti sanitari - definiti come Attività e procedure idonee a promuovere il miglioramento della qualità, efficienza, efficacia, appropriatezza e sicurezza.

Dopo una schizofrenica alternanza tra momenti di entusiasmo propositivo, sia legislativo che professionale, e lunghi periodi di assuefazione disinteressata, il Patto per la Salute conferma che la clinical governance è viva e vegeta: tuttavia, dopo oltre 15 anni dalla sua nascita, se da un lato fa sempre tendenza, dall'altro incute giustificati timori perché pone i professionisti sanitari al centro del sistema, ${ }^{21}$ rivoluzionando una logica aziendalista troppo impegnata alla produzione di servizi e prestazioni sanitarie, senza tenere conto della loro efficacia e appropriatezza. Inoltre, la clinical governance impone a tutti i professionisti sanitari sia si acquisire nuove competenze $\mathrm{e}^{22}$ - non ancora previste dai programmi di formazione universitaria e specialistica - sia soprattutto di essere accountable in tutte le prescrizioni diagnostico-terapeutiche-assistenziali, rinunciando ad autonomie professionali e interessi corporativi oggi non più sostenibili.

Il Servizio Sanitario Nazionale ha da poco compiuto 35 anni nell'indifferenza generale: ${ }^{23}$ il silenzio delle Istituzioni è stato assordante, ma assolutamente 
coerente con la linea abbracciata dalla politica che, dietro l'alibi del Titolo V, ha rinunciato a ogni forma di programmazione sanitaria, subordinando il diritto costituzionale alla salute alla crisi finanziaria del Paese. Il Ministero dell'Economia e delle Finanze ha progressivamente stretto il nodo della cravatta e il Governo, sbarazzatosi di una quota di spesa pubblica destinata alla Sanità, sembra aver imboccato senza troppi clamori la strada dell'intermediazione assicurativa e finanziaria dei privati, per garantire ossigeno a un settore la cui governance è da tempo sfuggita di mano allo Stato. In questo scenario particolarmente critico, con un Governo in grado di tenere a freno la spesa sanitaria attraverso tagli lineari, ma sinora incapace di attuare riforme già esistenti e di riprendere il dialogo con le Regioni per garantire un federalismo solidale senza derive regionaliste, i professionisti sanitari appaiono spettatori innocenti di fronte allo smantellamento del SSN e incapaci di qualunque reazione propositiva. Le categorie professionali variamente schiacciate tra contingenti necessità di contenere $\mathrm{i}$ costi, irrealistiche aspettative dei cittadini e assillanti timori medico-legali, non riescono a riprendere in mano le redini della Sanità in un ottica multi-professionale, basata sulle evidenze e centrata sul paziente, ma continuano a sprecare energie in lotte senza quartiere per mantenere i privilegi acquisiti senza rinunciare a nulla per la causa comune, testimonianza di corporazioni estremamente competitive e poco collaborative.

L'approccio di sistema alla clinical governance rappresenta senza alcun dubbio un passaggio obbligato per salvare il nostro SSN: adesso che anche il Patto per la Salute ne ha legittimato definitivamente la rilevanza non esiste più alcun alibi per tutti i professionisti sanitari che devono solo accantonare i propri interessi di categoria e aprire una nuova stagione di collaborazione, contribuendo a salvaguardare la più grande conquista sociale dei cittadini italiani, un servizio sanitario pubblico, equo e universalistico.

\section{Bibliografia}

1. Cartabellotta A. Il Servizio Sanitario Nazionale: una conquista sociale irrinunciabile. Huffington Post, 7 luglio 2013. Disponibile a: www.huffingtonpost.it/ninocartabellotta/il-servizio-sanitario-nazionale-una-conquis ta-sociale-irrinunciabile_b_3550305.html Ultimo accesso: 25 agosto 2014.

2. OECD Health Data 2014. Dove si colloca l'Italia? June 2013. Disponibile a: www.oecd.org/els/health-systems/Briefing-Note-ITALIA-2014-in-Italian.pdf Ultimo accesso: 25 Agosto 2014.

3. Hussey PS, Wertheimer S, Mehrotra A. The association between health care quality and cost: a systematic review. Ann Intern Med 2013;158:27-34.

4. Ministero della Salute. Patto della salute 2014-2016, intesa
Stato Regioni. Disponibile a: www.salute.gov.it/portale/ news/p3_2_1_1_1.jsp?lingua=italiano\&menu=notizie\&p $=$ dalministero\&id=1654 Ultimo accesso: 25 Agosto 2014.

5. Osservatorio Nazionale sulla Salute nelle Regioni Italiane. Rapporto Osserva Salute 2013. Disponibile a: http://www.osservasalute.it/index.php/rapporto/argomenti/2013/13. Ultimo accesso: 25 Agosto 2014.

6. Brody H. From an ethics of rationing to an ethics of waste avoidance. N Engl J Med 2012;366:1949-51.

7. Elshaug AG, McWilliams JM, Landon BE. The value of low-value lists. JAMA 2013;309:775-6.

8. Baker DW, Qaseem A, Reynolds PP, et al. Design and use of performance measures to decrease low-value services and achieve cost-conscious care. Ann Intern Med 2013;158:55-9.

9. Garner S, Littlejohns P. Disinvestment from low value clinical interventions: NICEly done? BMJ 2011;343:d4519.

10. Elshaug AG, Watt AM, Mundy L, Willis CD. Over 150 potentially low-value health care practices: an Australian study. Med J Aust 2012;197:556-60.

11. Shaw D, Elger B. Evidence-based persuasion: an ethical imperative. JAMA 2013;309:1689-90.

12. Tilburt JC, Cassel CK. Why the ethics of parsimonious medicine is not the ethics of rationing. JAMA 2013;309:773-4.

13. Geddes da Filicaia M. Cliente, paziente, persona. Ricerca e Pratica 2012;28:28-9.

14. Cartabellotta A. Il paziente è soddisfatto? Sì, ma costa troppo e sta peggio. Evidence 2012;4:e1000010.

15. Berwick DM, Hackbarth AD. Eliminating waste in US health care. JAMA 2012;307:1513-6.

16. Mathias JS, Baker DW. Developing quality measures to address overuse. JAMA 2013;309:1897-8.

17. FADOI. Master II Livello in Clinical Governance. Disponibile a: www.fadoi.org/formazione_master_secondo_livello Ultimo accesso: 25 Agosto 2014.

18. Ministero della Salute. Approvato dalla Conferenza Stato-Regioni il Regolamento per la definizione degli standard dell'assistenza ospedaliera. Disponibile a: www.salute.gov.it/portale/news/p3_2_1_1_1.jsp?lingua $=$ italiano $\&$ menu $=$ notizie $\& \mathrm{p}=$ dalministero $\& \mathrm{id}=1694$ Ultimo accesso: 25 Agosto 2014.

19. Ministero della Salute. Schema di Piano Sanitario 20112013. Disponibile a: www.statoregioni.it/Documenti/ DOC_033515_88\%20CU\%20PARTE\%201.pdf Ultimo accesso: 25 Agosto 2014.

20. Ministero della Salute. Nuovo sistema di formazione continua in medicina. Disponibile a: http://ape.agenas.it/documenti/Normativa/Il_nuovo_sistema_di_formazione_co ntinua_in_medicina.pdf Ultimo accesso: 25 Agosto 2014.

21. Degeling PJ1, Maxwell S, Iedema R, Hunter DJ. Making clinical governance work. BMJ 2004;329:679-81.

22. Fondazione GIMBE. Clinical Governance core curriculum. Disponibile a: www.gimbeducation.it/pagine/641/ it/curriculum Ultimo accesso: 25 Agosto 2014.

23. Cartabellotta A. Quel compleanno del SSN ignorato dalla politica e dimenticato dai professionisti. Il Sole 24 ORE Sanità; 21-27 gennaio 2014, pp 18-19. Disponibile a: www.gimbe.org/report_attivita/pubblicazioni/articoli/20140121-S24HS_AdP25-imp1.pdf Ultimo accesso: 25 Agosto 2014. 


\title{
È ancora possibile una reale alleanza tra clinici e gestori per una Medicina Interna ospedaliera sostenibile?
}

\author{
Stefano De Carli \\ SOC $2^{\text {a }}$ Medicina Interna, AOU S. Maria della Misericordia, Udine, Italia
}

\begin{abstract}
RIASSUNTO
L'analisi dell'incremento della spesa sanitaria deve distinguere tra quanto deriva dai meri costi inflattivi e da quanto invece è destinato a rispondere a nuovi bisogni di salute. L'incremento della spesa sanitaria deve inoltre essere rapportata all'andamento del prodotto interno lordo che sostiene il finanziamento della spesa sanitaria, attualmente in stagnazione. Le contromisure fin qui adottate in Italia per il contenimento della spesa hanno portato all'adozione di politiche di tagli lineari che, non solo non solo non hanno risolto il problema, ma aggravano ulteriormente la crisi di sostenibilità del Sistema Sanitario. L'adozione di politiche di riduzione mirata e di razionalizzazione passa attraverso un'analisi di appropriatezza sia clinica che organizzative del sistema salute. In questa prospettiva il medico (e l'internista in particolare) gioca un ruolo fondamentale essendo il promotore dell'appropriatezza clinica e il cardine di quella organizzativa, grazie alla funzione di ponte fra i pazienti e le istituzioni. L'acquisizione di queste competenze e ruoli vengono promossi tramite l'apprendimento di strumenti di clinical governance e risultano più efficaci nel professionista medico già formato rispetto a quello in formazione. In una sanità che cambia, la Medicina Interna, gioca un ruolo chiave, perché è presente in tutte le realtà ospedaliere italiane, è multidisciplinare e può ricoprire quel ruolo di interfaccia e coordinamento con i colleghi, il territorio e gli organi gestionali. Un limite importante all'interazione costruttiva dei clinici con i decisori aziendali è legato alla definizione di obiettivi di dettaglio che avviene in ambito nazionale e regionale, indebolendo il mandato dei Direttori Generali. Emerge tuttavia il ruolo potenziale delle Associazioni Scientifiche (come la FADOI) nell'orientamento e nella realizzazione di politiche sanitarie efficaci.
\end{abstract}

\section{Introduzione}

Negli ultimi decenni si è osservato un progressivo incremento della spesa in sanità attribuibile solo parzialmente a fenomeni inflattivi. Vanno infatti anche considerati i cambiamenti demografici legati all'invecchiamento della popolazione, le tendenze di frammentazione dei nuclei familiari e di aumento dell'isolamento sociale, i flussi migratori e di urbanizzazione, l'avvento di nuove tecnologie che modificano la pratica clinica e il progressivo emergere dei contenziosi medico-legali.

Tutte queste componenti fanno emergere oggi più che mai l'importanza della sostenibilità del sistema sanitario.

Corrispondente: Stefano De Carli, SOC $2^{\mathrm{a}}$ Medicina Interna, AOU S. Maria della Misericordia, Udine, Italia.

E-mail: decarli.stefano@aoud.sanita.fvg.it

Parole chiave: Medicina Interna, clinical governance, spesa sanitaria, appropriatezza, sostenibilità.

Articolo pubblicato secondo la Creative Commons Attribution NonCommercial 3.0 License (CC BY-NC 3.0).

CCopyright S. De Carli, 2014

Licensee PAGEPress, Italy

QUADERNI - Italian Journal of Medicine 2014; 2:95-100
Un sistema sanitario è sostenibile se le prestazioni fornite sono in grado di soddisfare i bisogni presenti senza compromettere la possibilità delle generazioni future di soddisfare i propri. Attualmente subiamo le conseguenze del welfare degli anni'60-90, basandosi sull'assioma poi rivelatosi errato, che sarebbe sempre stata presente una percentuale di popolazione attiva superiore a quella anziana ed in grado di finanziare lo stato previdenziale.

L'inversione delle dinamiche di crescita della popolazione, correlate con le dinamiche di andamento del rapporto tra la spesa sanitaria e il prodotto interno lordo (PIL), evidenziano un notevole rallentamento della crescita del rapporto negli ultimi vent'anni in Europa, e in modo ancor più marcato in Italia; elemento questo che ha condizionato la possibilità di finanziare l'assistenza sanitaria. ${ }^{1,2}$

Preso atto delle dinamiche di aumento dei costi, non essendo prevedibile una ripresa a breve/medio termine del PIL, è necessario attuare misure volte al contenimento della spesa in sanità.

Tra le molteplici scelte, le opzioni maggiormente accreditate sono: i) una redistribuzione della spesa pubblica, allocando risorse di altre voci a quella sanitaria; ii) un trasferimento di parte della spesa a carico del cittadino (il così detto out of pocket); iii) la razionalizzazione della spesa sanitaria; iv) la riduzione delle risorse destinate alla sanità.

Quest'ultima soluzione è stata intrapresa dai Go- 
verni che si sono succeduti negli ultimi anni, applicando tagli lineari alla spesa sanitaria, senza tuttavia portare benefici apprezzabili anche in termini di risparmio.

Un confronto del rapporto PIL/spesa sanitaria con altri paesi dell'area europea comparabili in termini di numerosità e composizione della popolazione (Germania e Francia), evidenzia come il rapporto $\mathrm{PIL} /$ spesa pubblica sia di poco superiore a quello dell'Italia, corrispondendo però, in termini assoluti, ad una spesa sanitaria pubblica effettiva pro-capite significativamente maggiore, cioè del $30 \%$ e del $23 \%$, rispettivamente. $^{2}$ Questo suggerisce che sarà difficile riuscire a comprimere ulteriormente i costi sanitari senza incidere sulla qualità dei servizi.

Una soluzione virtuosa al problema prevede di razionalizzare la spesa intervenendo chirurgicamente sulle inefficienze, ovvero riducendo gli interventi inappropriati.

In sanità possono essere descritte due tipologie di appropriatezza: i) professionale (individuale o clinica), che prevede esclusivamente la prescrizione di indagini o interventi terapeutici di efficacia provata (vengono evidenziati criteri di efficacia); ii) organizzativa (o gestionale) che si ottiene quando l'intervento o il percorso clinico viene erogato dalle strutture deputate secondo tempistiche idonee e di efficienza operativa.
È previsto che anche i concetti di appropriatezza possano modificarsi nel tempo per cui interventi ritenuti un tempo corretti possono essere successivamente rivisti alla luce di nuove evidenze. Nel campo dell'appropriatezza clinica possiamo ricordare alcuni esempi passati come: i) il trattamento delle problematiche post menopausali con estero-progestinici; ${ }^{3}$ ii) i programmi di screening dell'adenocarcinoma della prostata; ${ }^{4}$ iii) e più di recente, la rivisitazione degli outcome dello screening mammografico alla luce delle innovazioni dell'health technology assessment rispetto alle evidenze iniziali, svolta in Svizzera. Le attuali migliori possibilità di intervento in tutte le fasi della neoplasia sembrano infatti meno onerosi, comparabili in termini di sopravvivenza e migliori per la qualità di vita delle pazienti. ${ }^{5}$

La sistematizzazione di quest'ultima tipologia di intervento è la frontiera con cui i professionisti sanitari devono comunque confrontarsi quotidianamente: è noto che l'incremento delle risorse in sanità, non corrisponde ad un miglioramento delle condizioni di salute della popolazione. In tutti i sistemi si osserva che gli investimenti iniziali producono sensibili miglioramenti di outcome (incremento della sopravvivenza e della qualità dei servizi sanitari), superata una certa soglia (circa \$ 3000 pro capite) l'incremento di risorse determina benefici minimi, ${ }^{6}$ o che (Figura 1) possono

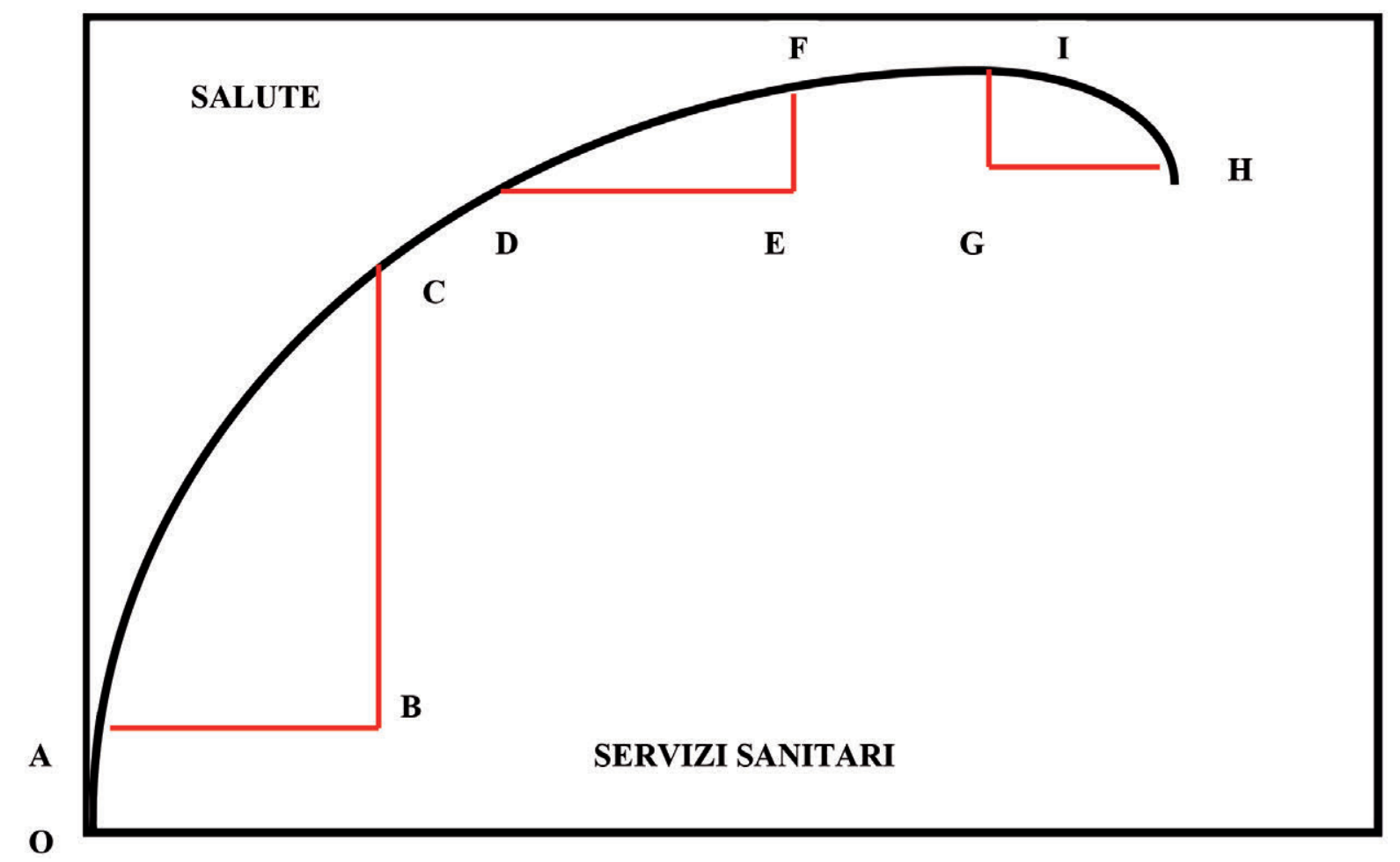

Figura 1. Il segmento OA rappresenta il livello di salute posseduto dalla popolazione in assenza di servizi sanitari. Le dosi iniziali di servizi sanitari $(\mathrm{AB})$ producono un incremento del livello di salute $(\mathrm{BC})$ di gran lunga più elevato di quello prodotto dalle successive dosi di servizi. Un ulteriore incremento di servizi sanitari (GH) potrebbe essere controproducente e portare a un peggioramento dell'outcome salute (IG). 
portare, paradossalmente, addirittura ad un peggioramento degli esiti (l'eccesso di prestazioni può condurre all'errore clinico, all'esecuzione di prestazioni affrettate quindi di bassa qualità, all'aumento dell'occorrenza di falsi positivi e all'aumento dell'occorrenza di effetti indesiderati ${ }^{7}$ ). Un altro motivo per cui l'impegno di risorse non crea necessariamente salute dipende dalla caratteristica peculiare della sanità per cui, contrariamente ad altri ambiti, l'innovazione tecnologica non si associa a riduzione dei costi, ma a una loro espansione. Inoltre in medicina non tutto ciò che è nuovo è innovativo (ad esempio i chemioterapici in oncologia ${ }^{8}$ ) e non tutto ciò che è vecchio è obsoleto (la metformina è tuttora farmaco di prima scelta nel trattamento del diabete). Inoltre, non tutto ciò che è innovativo è sostenibile (ad esempio i nuovi farmaci anti-HCV che non possono essere prescritti a tutti, pena il default del SSN).

\section{Appropriatezza, sostenibilità e ruolo del medico}

Appropriatezza e sostenibilità sono tematiche che non coinvolgono solo medici e gestori, ma anche i pa- zienti e tutta la collettività. Tutti sono componenti del sistema sanitario e ciascuno è portatore di interessi, perseguendo qualità valoriali specifiche. Queste ultime rappresentano una ricchezza nel dialogo fra le parti, ma possono, quando estremizzate, generare inappropriatezze ed essere centrifughe rispetto all'obiettivo della sostenibilità (Figura 2).

Proprio in questa rete di relazioni il medico funge da ponte fra i pazienti e i gestori (politici e amministratori), attraverso il rapporto di agenzia con il ruolo bivalente di tutelare il paziente nella scelta informata e di tutela del sistema sanitario garantendone gli obiettivi di sostenibilità e di universalità.

Oltre alle declinazioni territoriali che vedono il coinvolgimento dei Medici di Medicina Generale, anche in altri ambiti i professionisti medici devono trovare quotidiana realizzazione. Il caso delle dimissioni difficili è emblematico: sono situazioni in cui i pazienti, superata la fase acuta, non sono autonomi per tornare a casa, ma mancano spesso strutture in grado di gestirne la condizione di subacuzie (ex: RSA). Anche questa situazione infatti, pur rappresentando un esempio classico di inappropriatezza organizzativa, è una situazione in cui il medico (in qualità di profes-

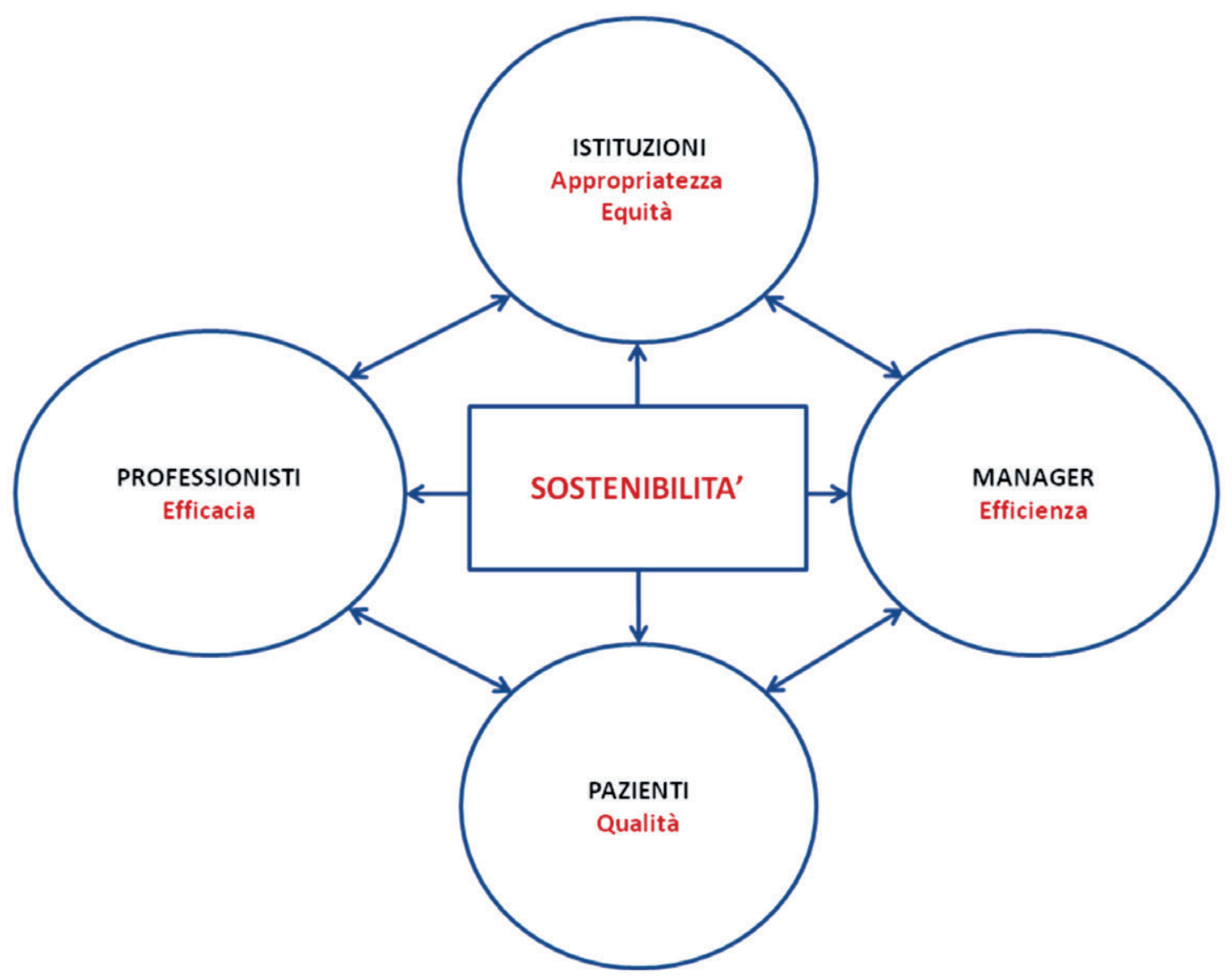

Figura 2. Obiettivo sostenibilità: soggetti coinvolti. 
sionista fiduciario delle parti) è l'unico attore in grado di dare effettiva risposta alle esigenze di salute tutelando le risorse comuni e gli interessi del paziente (anche facendogli comprendere il rischio di maggior esposizione al rischio infettivo, alla riduzione dell'autonomia fisica e di istituzionalizzazione associati con la permanenza nel reparto di cura).

L'attuale crisi della figura del medico (e dell'internista in particolare) emersa a seguito dei mutamenti delle scienze mediche, della società e dei modelli organizzativi, trova esplicitazione in un editoriale apparso nel 1983 sul British Medical Journal. ${ }^{9}$ L'autore, commentando i recenti progressi della terapia di rivascolarizzazione coronarica, osservava che si stavano affermando tecnologie innovative, in continua evoluzione che comportavano costi sempre più elevati per cui le decisioni dei clinici dovevano essere supportate da solide evidenze scientifiche, modificando il concetto di professione medica e dell'agire medico che non era più il solo depositario del processo di cura. Soprattutto in situazioni di particolare complessità, viene richiesta al curante la capacità di collaborare con tutte le altre figure sanitarie. A questo ha inoltre contribuito il venir meno del tradizionale rapporto paternalistico con l'assistito; il paziente è frequentemente già informato quando si reca dal medico, e sempre più frequentemente mal informato (a causa di fonti di dubbia qualità $)^{10} \mathrm{e}$ si afferma la consapevolezza di dover essere il decisore delle proprie scelte.

Il rapido sviluppo delle conoscenze richiede aggiornamenti continui, ma il moltiplicarsi delle fonti informative sottolinea, oggi più che mai, la differenza tra il professionista in grado di selezionare criticamente le fonti e l'utente disorientato da un bombardamento multimediale di informazioni raramente coerenti.

\section{Quale collaborazione è possibile fra decisori e internisti nella sanità che cambia?}

Nel difficile quadro sopra delineato, la clinical governance $(\mathrm{CG})$ può rappresentare uno strumento utile per catalizzare un dialogo tra professionisti sanitari e gestionali nella sanità.

Dobbiamo riconoscere che medici e gestori hanno una differente visione della sanità per mentalità e formazione (Tabella 1).

Il medico: i) è maggiormente orientato all'azione; ii) è orientato all'interazione con il singolo paziente; iii) ha un modus operandi reattivo; iv) si identifica maggiormente con la professione e ritiene fondamentali la propria autonomia e indipendenza.

Per converso, il manager: i) pianifica; ii) è proattivo; iii) considera gruppi di popolazioni e ritiene fondamentali la collaborazione, la partecipazione ai lavori di gruppo e si identifica con le strutture organizzative. ${ }^{11}$

Le relazioni di coordinamento tra manager e medici che sono gerarchiche e di natura formale sono più deboli di quelle informali e collaterali tra medici (anche quando appartenenti a branche e strutture differenti), poiché condividono conoscenze, valori e codice etico.

In uno studio pubblicato a metà degli anni ' 90 era stata proposta la seguente simulazione a pazienti, medici e manager della sanità: il governo vuole offrire a tutta la popolazione di più di 40 anni uno screening per il cancro al colon il budget per questo programma è limitato a $\$ 200.000$. Sono disponibili 2 test: il Test 1 costa $\$ 200.000$ e può quindi essere offerto a tutti e eviterà 1000 decessi, il Test 2 costa $\$ 400.000$ e può quindi essere dato al $50 \%$ della popolazione eleggibile (scelta casuale). È però più efficace, perché eviterebbe 1100 decessi.

Solo i manager della sanità rispondevano in prevalenza che era più importante il miglior risultato raggiunto con il Test 2 rispetto all'equità apparente del Test $1 .{ }^{12}$

Tramite l'apprendimento degli strumenti di CG il medico assume competenze che riducono le caratteristiche peculiari strettamente cliniche che tendono ad ostacolare la capacità di integrarsi nelle organizzazioni sanitarie. È opinione di esperti delle organizzazioni sanitarie che tanto più sono le competenze cliniche sviluppate, tanto migliori saranno le possibilità di apprendere strumenti di tipo manageriale. La conoscenza di questi strumenti connota il medico differen-

Tabella 1. Medici e gestori: due differenti visioni della sanità per mentalità e formazione.

\begin{tabular}{ll}
\hline Medico & Manager \\
\hline Orientato all'azione & Pianificatore \\
\hline Interazione $1: 1$ & Interazione 1:N \\
\hline Professionalità reattiva & Professionalità proattiva \\
\hline Richiede immediata gratificazione & Accetta gratificazione ritardata \\
\hline Autonomia = Valore & Collaborazione = Valore \\
\hline Indipendente & Partecipativo \\
\hline Orientato al paziente & Orientato all'organizzazione \\
\hline Identificato con la professione & Identificato con l'organizzazione \\
\hline
\end{tabular}


ziandolo, per mentalità, dai colleghi professionisti puri (Figura 3). ${ }^{13,14}$

L'internista, per l'approccio multidisciplinare ha una visione differente rispetto agli altri specialisti ed è quindi avvantaggiato nell'acquisizione degli strumenti di CG e nel dialogo con i gestori.

In molte realtà sanitarie europee, come Norvegia, Danimarca, Olanda ${ }^{15}$ sono già presenti assetti organizzativi che prevedono una stretta collaborazione fra clinici e gestori che favoriscono processi sanitari efficienti ed efficaci, che generano appropriatezza, premessa necessaria per la sostenibilità (Figura 4).

Anche il medico che non conosce i rudimenti della $\mathrm{CG}$, quando propone ai propri assistiti una o più opzioni disponibili, opera, benché inconsapevolmente, delle selezioni (scelte) che hanno impatti economici. Pertanto è opportuno la $\mathrm{CG}$ diventi patrimonio comune. ${ }^{16}$

Le organizzazioni sanitarie moderne chiedono inoltre al medico di rinunciare parzialmente alla propria indipendenza, che non deve essere vista come un limite perchè l'acquisizione di nuove competenze di CG gli offre nuove opportunità e potenzialità. In particolare vengono stimolate capacità di porsi in modo proattivo, avvicinandolo e facilitandone la collaborazione e il dialogo con i gestori nella realizzazione di obiettivi di sostenibilità (budget). Vengono stimolate e accresciute le sue capacità di collaborazione con gli altri professionisti sanitari migliorando l'approccio multi-disciplinare e multi-professionale al singolo caso clinico, ${ }^{17}$ nella formazione di un percorso di cure personalizzato.

Un internista che possieda capacità cliniche e organizzative di CG può svolgere da ponte fra gestori e pazienti educando questi ultimi e guidandone le scelte nel soddisfare il bisogno di salute; facendo emergere i bisogni reali, soddisfabili, da quelli percepiti o indotti. La costruzione di un dialogo che favorisca il rapporto di fiducia medico-paziente rinsalda anche quella fra il paziente e il sistema sanitario e riduce le occasioni di conflitto, motivo più frequente di cause legali in sanità.

\section{La medicina interna ospedaliera nel sistema sanitario}

La Medicina Interna ha tutte le potenzialità per essere strategica per i decisori in quanto i reparti di Medicina Interna sono presenti in tutte le strutture

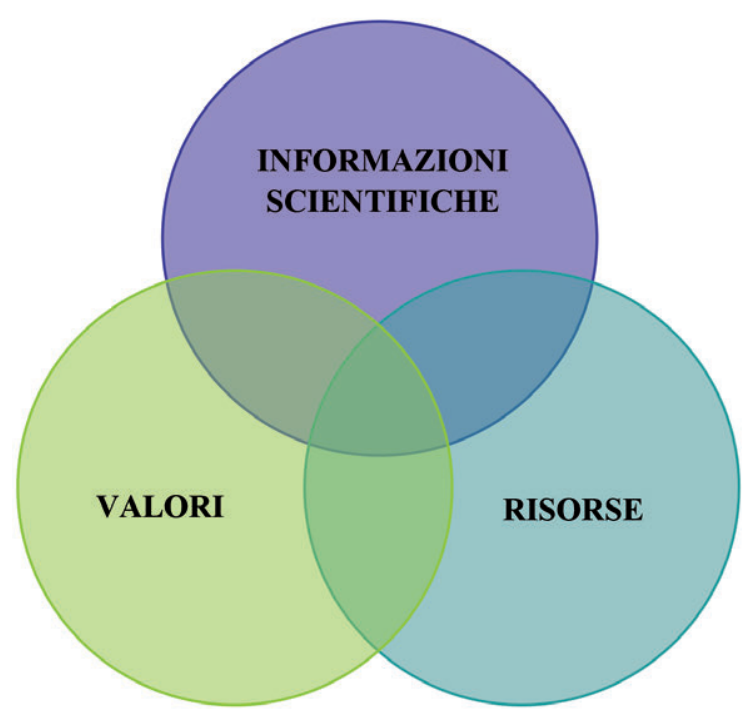

Figura 3. Le componenti caratterizzanti il medico con formazione di clinical governance.

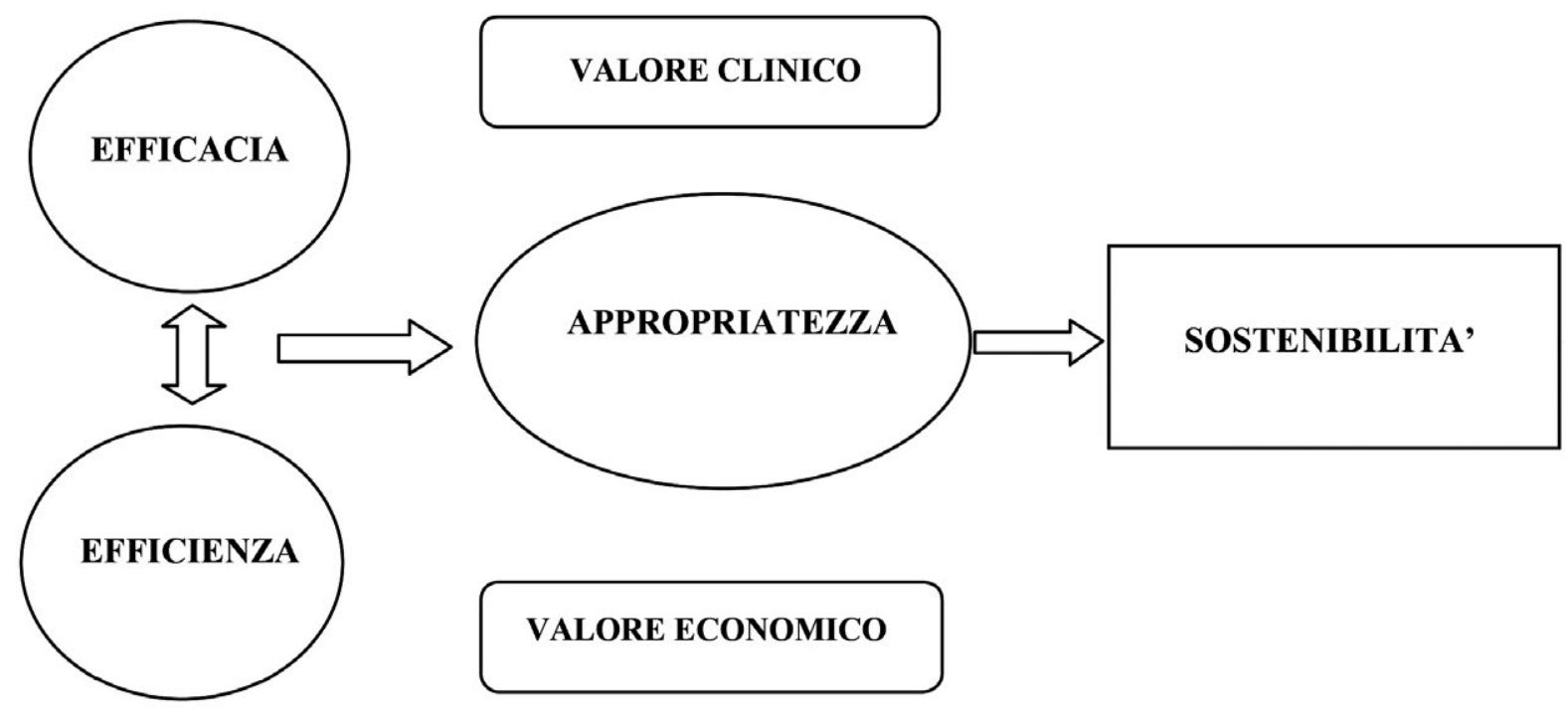

Figura 4. L'appropriatezza come premessa necessaria per la sostenibilità. 
ospedaliere. L'approccio per processi tipico della medicina interna favorisce l'interazione con le altre figure sanitarie (medico-specialistiche e infermieristiche) ospedaliere e territoriali. La naturale predisposizione culturale, professionale e formativa dell'Internista lo porta a una visione multidisciplinare e trasversale. Tutto ciò gli permette di acquisire con maggiore facilità gli strumenti che compongono la struttura della CG e lo facilita nel dialogo con i decisori della sanità.

L'Internista ospedaliero, collaborando con i gestori, ha quindi un ruolo fondamentale nel permettere la sostenibilità del Sistema Sanitario nazionale in epoca di carenza di risorse. Anche in sistemi sanitari privatistici come negli Stati Uniti viene riconosciuta alla Medicina Interna la capacità, in un contesto organizzato, di ridurre il burden delle patologie croniche e delle riacutizzazioni, effetto che dovrebbe avere maggiore impatto in contesti, come l'Italia, dove il sistema sanitario è pubblico e nazionale. ${ }^{18}$

\section{Problematiche aperte}

Rimangono tuttavia numerosi problemi aperti che ostano il raggiungimento del traguardo della sostenibilità. L'attuale assetto ospedaliero (organizzativo e anche edilizio), pur con i vari tentativi di modelli per intensità di cura è ancora strutturato secondo approcci datati. Il progressivo impoverimento di specialisti nelle strutture sanitarie a cui stiamo assistendo in questi anni, sta portando al progressivo accorpamento delle strutture specialistiche nei reparti internistici. Una possibile evoluzione delle figura dell'internista è di assumere negli ospedali per intensità di cure un ruolo analogo a quello dell'hospitalist delle realtà anglosassoni (dove è quasi sempre un internista) gestendo il paziente ospedaliero in tutte le fasi di cura, ponendosi in prima persona in relazione con il Medico di Medicina Generale. Le attuali relazioni tra ospedale e territorio sono ancora largamente insufficienti ed è mandatorio trovare una risposta organizzativa strutturale che passa attraverso i reparti di medicina interna. L'attuale tendenza che vede la programmazione sanitaria ancorata alla definizione di obiettivi di dettaglio sia in ambito nazionale che regionale, riduce il potere dei decisori locali (Direttori Generali). Questo comporta un limite non trascurabile alla possibilità dei clinici di agire in senso propositivo per migliorare gli assetti organizzativi locali (micro), ruolo che invece può essere giocato dalla FADOI in quanto Società Scientifica in ambito meso (Regionale) e macro (nazionale).

\section{Bibliografia}

1. Ambrosetti Club. L'evoluzione sostenibile della sanità in Italia. Lettera Maggio/giugno 2013:1-4.

2. Pammoli F, Salerno NC. Le proiezioni della spesa sanitaria del SSN 2011-2030. CERM. Working paper 2011/3.

3. Mansion JE, Hsia J, Johnson KC, et al. Estrogen plus progestin and the risk of coronary heart disease. N Eng1 J Med 2003;349:523-34.

4. Ilic D, Neuberger MM, Djulbegovic M, Dahm P. Screening for prostate cancer. Cochrane Database Syst Rev 2013;1:CD004720.

5. Biller-Andorno N, Jüni P. Abolishing mammography screening programs? A view from the Swiss Medical Board. N Engl J Med 2014;370:1965-7.

6. Mc Kinlay JB, Mc Kinlay SM. The questionable contribution of medical measures to the decline of mortality in the United States in the twentieth century. Mibank Memorial Fund Quart 1977;55:405-28.

7. Orszag PR, Ellis P. Addressing rising health care costs a view from the Congressional Budget Office. N Engl J Med 2007;357:1885-7.

8. Grady FC. How much is life worth: cetuximab, nonsmall cell lung cancer, and the $\$ 440$ billion question. $\mathrm{J}$ Natl Cancer Inst 2009;101:1044-8.

9. Hampton JR. The end of clinical freedom. Br Med J (Clin Res Ed) 1983;287:1237-8.

10. Diem SJ. Lantos JD, Tulsky JA. Cardiopulmonary resuscitation on television. Miracles and misinformation. N Engl J Med 1996;334:1578-82.

11. Kindig DA, Konver AR. The role of physician executive. Ann Arbor: Health Administration Press; 1992.

12. Ubel PA, DeKay ML, Baron J, Asch DA. Cost-effectiveness analysis in a setting of budget constraints. N Engl J Med 1996;334:1174-7.

13. Ferlie E, Pettigrew A, Ashburner, Fitzgerald L. The new public management in action. Oxford: Oxford University Press; 1996.

14. Cicchetti A. La progettazione organizzativa. Milano: Franco Angeli/Sanità; 2004.

15. Kuhlmann E, Burau V, Correia T, et al. "A manager in the minds of doctors": a comparison of new modes of control in European hospitals. BMC Health Serv Res 2013;13:246.

16. Jacobs VR, Fischer T. Pragmatic guide on how physicians can take over financial control of their clinical practice. JSLS 2012;16:632-8.

17. Prosser LA, Stinnett AA, Goldman PA, et al. Cost effectiveness of cholesterol-lowering therapies according to selected patient characteristics. Ann Int Med 2000;132:769-79.

18. Yale School of Medicine. Ambulatory care internal medicine has a critical role in the US health care system. Available from: https://medicine.yale.edu 


\title{
La cultura internistica come visione di un auspicato cambiamento in medicina e nella organizzazione ospedaliera: l'esperienza di due realtà toscane
}

\author{
Giancarlo Landini, ${ }^{1}$ Grazia Panigada, ${ }^{2}$ Luca Masotti, ${ }^{3}$ Irene Chiti ${ }^{2}$ \\ ${ }^{1}$ Dipartimento di Medicina e Specialistiche Mediche, Azienda Sanitaria di Firenze; ${ }^{2} \mathrm{SC}$ di Medicina Interna, Ospedale SS \\ Cosma e Damiano Pescia, Azienda USL3 Pistoia; ${ }^{3}$ SC di Medicina Interna, Ospedale Santa Maria Nuova, Azienda Sanitaria \\ di Firenze, Italia
}

\section{RIASSUNTO}

Le criticità della medicina ospedaliera si possono riassumere in due ordini di problemi: i) il sistema è centrato sulla cura delle malattie acute in un mondo in cui prevalgono le malattie croniche e croniche riacutizzate; ii) la metodologia clinica è orientata alla semplificazione dei problemi (riduzionismo) in una situazione in cui gli ammalati sono polipatologici e complessi con interazione inestricabile fra le varie patologie. La cultura ed il modus operandi della Medicina Interna possono essere molto utili per superare queste difficoltà. La Medicina Interna gestisce pazienti con co-morbilità che tendono a complicarsi ma più ancora pazienti complessi che presentano di solito multiple malattie croniche riacutizzate Il malato internistico è un malato acuto fragile che necessita spesso di un trattamento ad alta intensità e quindi rappresenta l'esempio tipico dei ricoverati nelle aree mediche dei nostri ospedali dove vengono gestiti pazienti ad alta complessità clinica e molte volte instabili.

\section{Il paziente complesso in medicina interna}

La complessità è stata definita molto bene da Edgar Morin come una situazione in cui le differenti componenti che costituiscono un tutto sono inseparabili e vi è una totale interdipendenza. ${ }^{1}$

Il paziente complesso è una persona affetta da due $o$ più malattie in cui ciascuna delle condizioni morbose presenti è in grado di influenzare l'esito della cura delle altre coesistenti con varie modalità.

Il paziente complesso è decisamente più difficile da affrontare rispetto al paziente complicato: quest'ultimo rappresenta il caso in cui ad una malattia se ne aggiunge un'altra che ne peggiora la prognosi. Il paziente complesso, come dice il nome, è un paziente che è intrecciato e quindi non si può spiegare come il complicato. ${ }^{2}$

La gestione di questo tipo di paziente porta

Corrispondente: Giancarlo Landini, Dipartimento di Medicina e Specialistiche Mediche, Azienda Sanitaria di Firenze, Italia. E-mail: giancarlo.landini@asf.toscana.it

Parole chiave: cultura internistica, organizzazione ospedaliera, FADOI Toscana.

Articolo pubblicato secondo la Creative Commons Attribution NonCommercial 3.0 License (CC BY-NC 3.0).

CCopyright G. Landini et al., 2014

Licensee PAGEPress, Italy

QUADERNI - Italian Journal of Medicine 2014; 2:101-105 l'internista ad operare in condizioni di incertezza: egli deve infatti saper affiancare alla medicina basata sulle evidenze (EBM) la medicina basata sul paziente. La EBM è esaustiva in Medicina Interna solo nel 19\% dei casi; per il resto le decisioni devono essere prese in base a condizioni fisiopatologiche, alle priorità emergenti, alle interazioni fra le malattie, alla propensione del paziente e dei familiari ed in considerazione del contesto normativo e sociale (Figura 1). ${ }^{3}$

A questo proposito sono significativi i 10 punti condivisi FADOI/Slow Medicine (Tabella 1) che mettono a fuoco bene il cambiamento di prospettiva dell'assistenza medica ospedaliera: secondo questo decalogo bisogna sempre fare una selezione delle priorità di intervento e devono essere sempre valutati sia il rapporto rischio/beneficio che l'utilità per l'assistito di richiedere un nuovo test o esame, non si deve prescindere dal coinvolgimento della persona, dall'attenzione alla qualità della vita e dal controllo del dolore. ${ }^{4}$

In questo panorama la Medicina Interna si propone come nuovo paradigma dell'assistenza in area medica dal momento che persegue la gestione del paziente acuto complesso attraverso un tutoraggio unitario del percorso di cura (diagnosi e terapia). In considerazione del tipo di malati che oggi accedono alle strutture ospedaliere è auspicabile che tale metodo venga adottato anche dalle altre specialità mediche per ottenere risultati efficaci.

Con queste premesse la Medicina Interna può essere l'asse portante di una area medica ospedaliera che garantisca il recupero della centralità del paziente, la 
flessibilità nell'uso delle risorse, il coordinamento delle specialistiche di organo e di patologia, il coordinamento dei piani di cura e la continuità dei percorsi.

Questa organizzazione unita ad un diverso utilizzo degli spazi di degenza in relazione alla domanda è uno dei punti di forza dell'ospedale per intensità di cura (Figura 2). ${ }^{5}$

\section{Medicina interna ed intensità di cura}

L'Ospedale per intensità di cura è centrato sui bisogni assistenziali e clinici dei pazienti e non sulle attività delle varie specialità mediche.

I punti qualificanti dell'intensità di cura sono: i) il reparto ricovera pazienti con necessità clinicoassistenziali simili; ii) gli infermieri sono assegnati al reparto e non alle UO ed esercitano competenze professionali autonome (Nursing Unit - cellule di assistenza); iii) i letti sono assegnati alle UUOO in modo flessibile; iv) l'assistenza è effettuata secondo processi unitari di cura e vi è una forte integrazione con il territorio secondo percorsi preordinati e standardizzati; v) l'assistenza è caratterizzata dalla multidisciplinarietà e multiprofessionalità con tendenza alla cogestione dei pazienti; vi) la Medicina Interna è la specialità che svolge la funzione di tessuto connettivo dell'area medica dell'ospedale. ${ }^{6}$

La Regione Toscana nel 2005 con la legge 40 aveva cominciato a parlare di intensità di cure $(\ldots$ tendere alla strutturazione delle attività ospedaliere in aree differenziate secondo le modalità assistenziali,l'intensità di cure, la durata della degenza e il regime del ricovero).

La Medicina Interna Toscana anche attraverso 1'Associazione degli Internisti Ospedalieri FADOI, forte della cultura olistica ed integrata della disciplina, ha colto subito le opportunità e i rischi di questo nuovo modello e si è proposta come interlocutore per gestire un cambiamento compatibile e sostenibile.

Dal primo articolo pubblicato sull'Italian Journal of Medicine nel $2007^{7}$ sono seguiti numerosi interventi che sono culminati nel 2010 nel documento delle società scientifiche toscane e nel 2013, nell'ambito dell'osservatorio per l'ospedale per intensità cure del CSR, nella definizione del percorso medico del paziente nell'ospedale attraverso la spiegazione dettagliata di un processo di cura integrato che tende a superare i livelli di assistenza. ${ }^{8}$

\section{Medicina interna e nuova organizzazione ospedaliera in Toscana}

In Toscana la nuova organizzazione ospedaliera ha impiegato diversi anni ad affermarsi ma ormai il quadro generale è ben delineato tant'è vero che i nuovi ospedali (Prato, Pistoia, Lucca e Massa) sono stati costruiti con una struttura pensata proprio per rispondere alle esigenze dell'intensità di cura.

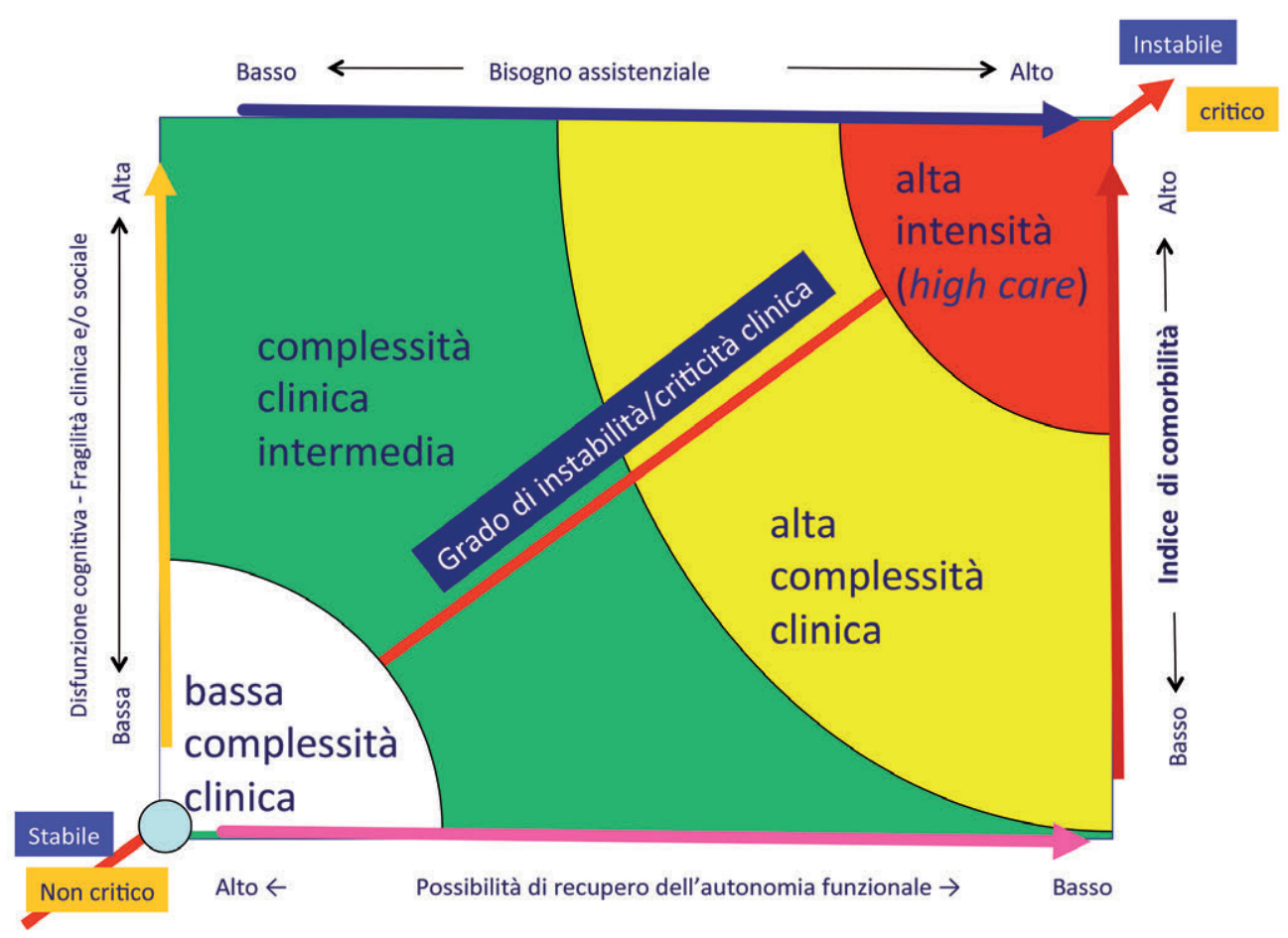

Figura 1. I 5 assi che definiscono la complessità assistenziale clinica in Medicina Interna. (Nardi et al., 20121). 
Nella Azienda Sanitaria di Firenze il processo è stato approfondito e ad oggi è stato completato. Nei 5 ospedali dell'Azienda all'interno dell'area medica denominata high care medica trovano posto le varie specialistiche con un numero flessibile di letti raggruppati geograficamente per evitare il rischio clinico della dispersione nei vari setting. Inoltre sono presenti aree speciali con congruenza verticale come l'Area Stroke e un'area a più alta intensità (subintensiva o area $2 \mathrm{~A}$ ) ove sono ricoverati pazienti con modified early warning score (MEWS) $>3$.

La gestione infermieristica avviene per cellule di assistenza secondo i concetti del primary nursing.

Tutte le aree mediche dell'azienda hanno una cartella clinica unica medico-infermieristica computerizzata.

Il tradizionale giro visita è stato sostituito da un briefing mattutino fra le varie professionalità, a cui segue una fase in cui il lavoro viene svolto in parallelo e che si conclude con un debriefing finale.

La guardia di continuità è effettuata dai medici afferenti alla Medicina Interna che svolgono sempre di più le funzioni di hospitalist.

A fianco delle degenze con attività $\mathrm{H} 24$ opera un Day Service multidisciplinare di area medica che permette una interfaccia diretta con il territorio coinvolgendo gli specialisti ambulatoriali e i Medici di Medicina Generale con percorsi bidirezionali preordinati e standardizzati con il fine di ridurre il ricorso al DEA e di arginare il problema del revolving door (rientro).

Una simile organizzazione è stata realizzata anche nell'azienda USL 3 di Pistoia e in particolare nell'ospedale di Pescia. Le caratteristiche strutturali

Tabella 1. I 10 punti FADOI/Slow Medicine.

\begin{tabular}{ll}
\hline 1. & Nel paziente internistico complesso va fatta una selezione delle priorità di intervento \\
\hline 2. & Non vanno richieste consulenze specialistiche ridondanti \\
\hline 3. & Valutare rischio/beneficio ed utilità per il paziente prima di richiedere un nuovo test o esame \\
\hline $4 . \quad$ & Informare e coinvolgere il paziente e familiari nelle scelte terapeutiche \\
\hline 5. & Nella gestione del fine vita valutare in primo luogo qualità della vita e il controllo del dolore \\
\hline 6. & Oculata valutazione dei farmaci da prescrivere \\
\hline 7. & Uso dei farmaci equivalenti \\
\hline 8. & Alla dimissione ridurre il numero dei farmaci prescritti \\
\hline 9. & Effettuare un piano di dimissione con rapporti preordinati con il territorio \\
\hline 10. & Implementare autocura e programma di follow-up \\
\hline
\end{tabular}

PSR 2008-2010: riorganizzazione del SSR: ospedale per intensità di cura

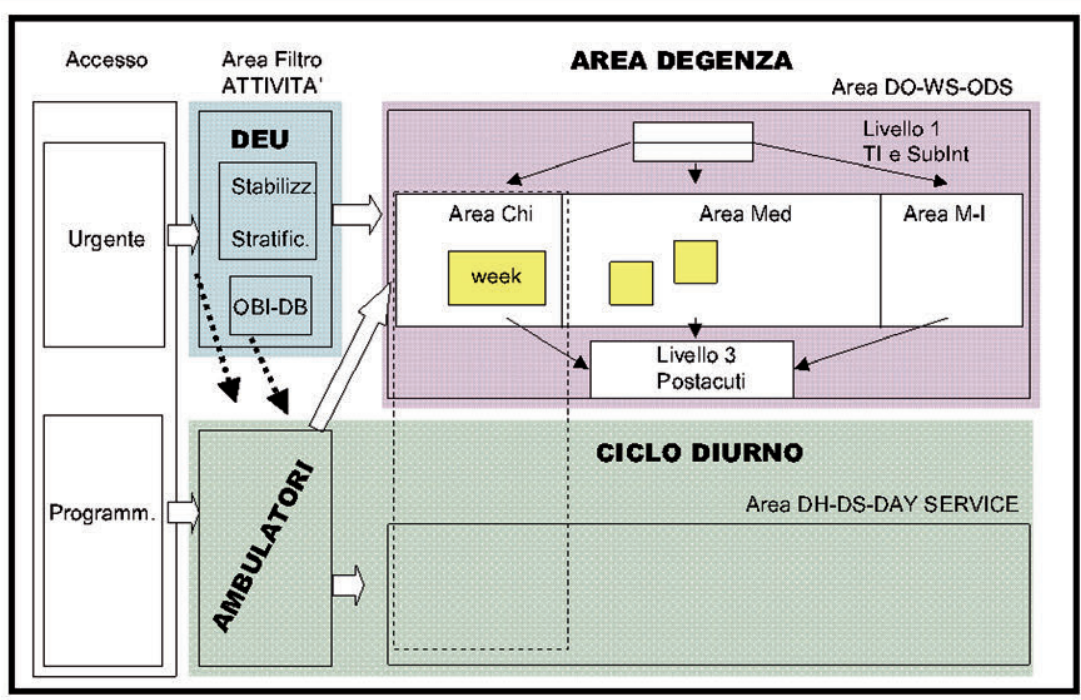

L'Ospedale si struttura per aree e livelli in base ad un fabbisogno assistenziale

Figura 2. Organizzazione e diverso utilizzo degli spazi di degenza come punti di forza dell'ospedale per intensità di cura. 
dell'ospedale, costituito da più padiglioni di differenti epoche storiche, non hanno però permesso l'inserimento anche dei letti di Cardiologia in area medica, mentre Medicina Interna e le altre specialistiche mediche utilizzano in maniera flessibile due setting. Rilevante la problematica del sovraffollamento che grava principalmente sulla Medicina Interna nonostante l'elevato numero di pazienti seguiti attraverso il Day Service e il collegamento in rete con l'ospedale di Pistoia che è stato costruito appositamente per l'applicazione del modello, ma che soffre anch'esso di sovraffollamento internistico.

Punto di forza è rappresentato dall'high care medica inserita nel setting A che, pur non presentando le caratteristiche normative di una sub intensiva, accoglie pazienti critici con MEWS $>3$ appartenenti a tutte le diverse discipline di area medica.

All'interno dell'High Care è presente l'Area Stroke a gestione internistica, dove viene implementata una clinical pathway definita con caratteristiche multi professionali e multidisciplinari (medica, infermieristica, fisioterapica) che permette di seguire $\mathrm{i}$ pazienti dalla fase iperacuta (con effettuazione anche della fibrinolisi sistemica) fino all'affidamento alla Riabilitazione Intensiva. $\grave{E}$ attivo anche un collegamento in rete con centri di terzo livello quando si rendano necessarie procedure più specialistiche (trombolisi loco regionale, neurochirurgia etc.).

Vengono così garantiti i principi della Stroke Unit senza tuttavia rappresentarne l'esclusività.

Nello stesso setting infatti trovano collocazione l'insufficienza respiratoria trattata con ventilazione non invasiva, la sepsi e lo shock settico che necessitano di competenze intensivistiche e spesso di algoritmi decisionali condivisi con il rianimatore, l'insufficienza renale acuta e patologie che richiedano l'emofiltrazione, la pancreatite acuta e le emorragie digestive L'assegnazione alla specialistica di competenza viene definita fin dal DEU in base a criteri predefiniti.

La Medicina Interna assorbe la stragrande maggioranza della casistica e funziona da catalizzatore perché sempre più numerosi sono i pazienti polipatologici complessi in cui il tutoraggio internistico si è dimostrato più efficace ed efficiente.

Tuttavia la presenza di diverse specialistiche nel medesimo setting, ha favorito da subito la condivisione dei casi, gli scambi culturali e formativi.

Il modello infermieristico applicato nell'Azienda di Pistoia ricalca quello fiorentino.

Gli strumenti che ne favoriscono l'applicazione sono similari anche se non disponiamo ancora della completa informatizzazione.

La guardia di continuità è effettuata dai medici afferenti alla Medicina Interna con l'esclusione di 4 notti al mese in cui al momento è garantita dalle altre specialistiche per compensare le carenze organiche

Anche a Pescia è attivo un Day Service che permette di applicare percorsi diagnostico terapeutici condivisi con il territorio al fine di ottimizzare il ricorso al ricovero ordinario e gestire a livello ambulatoriale anche patologie complesse (Figura 3).

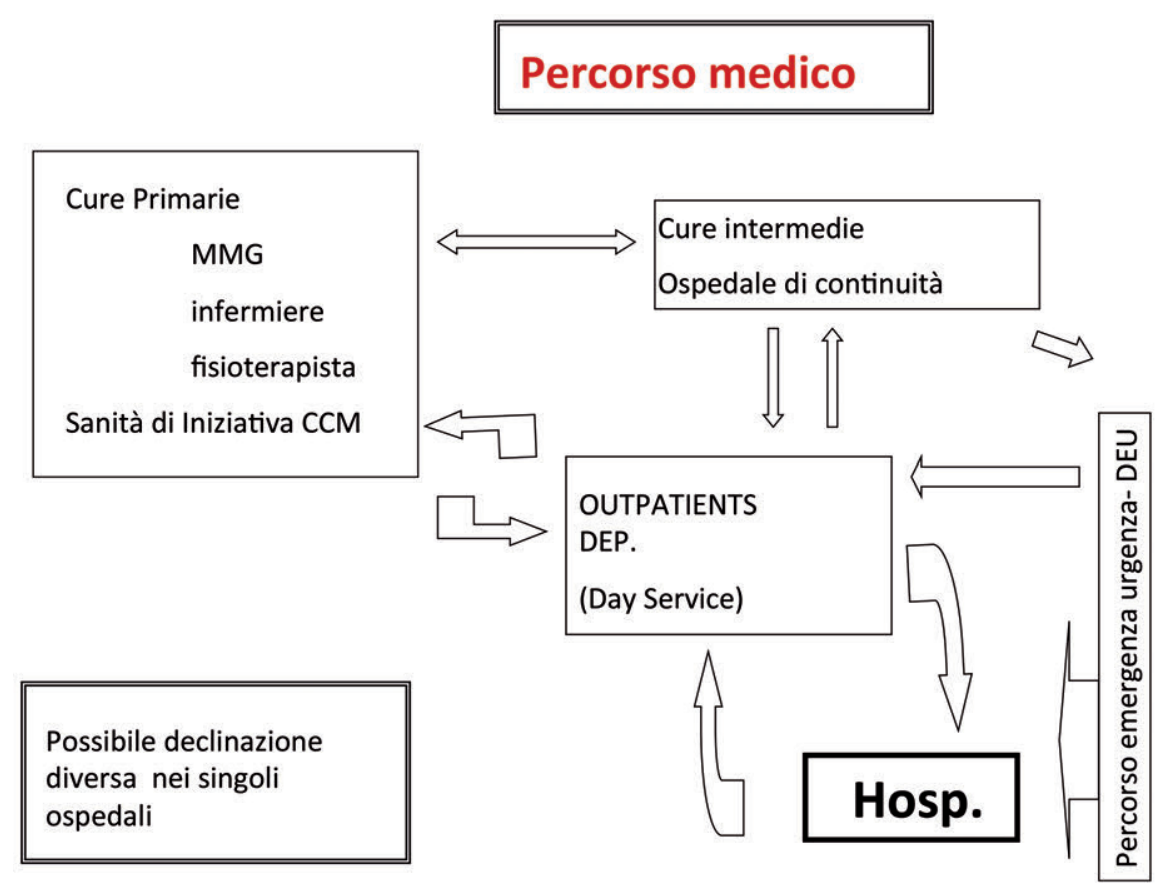

Figura 3. Percorso diagnostico terapeutico per ottimizzare il ricovero ordinario e la gestione delle patologie complesse. 


\section{Conclusioni}

La crisi economica, la complessità e polipatologia dei pazienti obbligano la sanità ad affrontare nuovi paradigmi gestionali.

L'ospedale per intensità di cura associato ad un nuovo tipo di rapporto ospedale territorio può essere la soluzione che mantiene i livelli di assistenza e genera appropriatezza e risparmio.

In questo contesto la Medicina Interna Ospedaliera ha visto progressivamente aumentare la propria attività e importanza per le caratteristiche culturali e di indirizzo che la specialità ha sempre avuto.

In questo contesto la Medicina Interna Ospedaliera ha visto progressivamente aumentare la propria attività e importanza per le caratteristiche culturali e di indirizzo che la specialità ha sempre avuto. Questo ruolo potrà essere mantenuto solo a fronte di un'adeguata crescita della competenza clinica e tecnico-professionale e della contestuale presa di coscienza del ruolo di governo clinico che le è demandato.

\section{Bibliografia}

1. Nardi R, Arienti V, Nozzoli C, Mazzone A. L'ospedale per intensità di cure: gli errori da evitare. Ital J Med 2012;6:1-13.

2. Ministero della Salute. Criteri di appropriatezza, clinica, tecnologica e strutturale nella assistenza del paziente complesso. Quaderni del Ministero della Salute n.23; Settembre-Ottobre 2013.

3. Nardi R, Fabbri T, Belmonte G, et al. Medicina Interna, paziente complesso, evidence-based medicine e le non evidenze. Ital J Med 2009;3:191-200.

4. D'Amore F. The doctor and the patient: doo into much o too little. Ital J Med 2013;7:135-7.

5. Cavicchi I. Medicina Interna, paziente complesso verso una clinica relazionale e ragionevole. Ital J Med 2012;6:259-64.

6. Niccolai F, Nuti S. Intensità di cura e intensità di relazioni. Pisa: Edizioni ETS; 2012.

7. Alessandri M, Bartolomei C, Bernardini M, et al. Medicina Interna e nuova organizzazione ospedaliera:le proposte di FADOI Toscana. Ital J Med 2007;1:35-7.

8. Cecchi M, Landini G, Andreini R, et al. Percorso medico ospedale per intensità di cure. Toscana Medica 2013;31:32-6. 


\title{
Indicatori di qualità nella Medicina Interna ospedaliera: solamente durata di degenza, ricoveri inappropriati, mortalità intraospedaliera e tasso di re-ricovero a $\mathbf{3 0}$ giorni?
}

\author{
Emanuela Foglia, ${ }^{1}$ Davide Croce ${ }^{1,2}$ \\ ${ }^{1}$ Centre for Research on Health Economics, Social and Health Care Management - CREMS, Carlo Cattaneo University - LIUC, \\ Castellanza (VA), Italy; ${ }^{2}$ School of Public Health, Faculty of Health Sciences, University of the Witwatersrand, Johannesburg, \\ South Africa
}

\section{RIASSUNTO}

In Sanità, l'attenzione verso i sistemi di programmazione e controllo è sempre viva: il superamento dei tradizionali modelli e l'implementazione di nuove modalità di tipo multidimensionale è, ormai, una esigenza sentita sia dalla componente clinica, sia da quella gestionale, eppure non viene univocamente ricercata. La Clinical Governance può rappresentare un buon modello, ma per divenire una pratica operativa necessita di una attenta pianificazione e comunicazione tra le differenti figure professionali sanitarie, nonché di una localizzazione rispetto alle differenti esigenze specialistiche. È necessario dunque agire in un'ottica multidimensionale e multidisciplinare, sostituendo la misurazione dell'output e della prestazione sanitaria, alla valutazione degli esiti generati, in termini di outcome e, quindi, anche di qualità. Solo un percorso di questa tipologia e l'affermarsi della clinical governance potranno effettivamente consentire un generale miglioramento dei servizi, con una riduzione delle spese per il Servizio Sanitario Nazionale. Tutto ciò necessita, però di un buon sistema di connessione con il percorso di programmazione e controllo, quindi, di budget. In questo contesto, sono sufficienti indicatori che vanno a monitorare la durata media della degenza e il tasso di re-ricovero a 30 giorni? Oppure è possibile fare qualcosa di più? Si cercherà di rispondere a questa domanda, attraverso una digressione, dove la qualità diventerà uno strumento e un vero e proprio personaggio, non solo tecnico, ma soprattutto gestionale.

\section{Introduzione}

C'era una volta il D. Lgs. 502, che nel lontano $1992^{1}$ sconvolse il regno di Sanità, con una serie di strumenti di monitoraggio (tra i quali il famigerato budget), volti al controllo delle attività erogate a favore dei cittadini/sudditi, del rimborso di queste prestazioni, nonché della temibile strega Spesa Sanitaria, che imperversava gettando tutti nello sconforto e nella paura.

Nonostante le modalità con le quali il D. Lgs. è stato implementato non fossero sempre ortodosse e tecnica-

Corrispondente: Emanuela Foglia, corso Matteotti 22, 21053

Castellanza (VA), Italia.

Tel.: +39.0331.572.516 - Fax: +39.0331.572.513

E-mail: efoglia@liuc.it

Key words: clinical governance, indicatori, sistemi di controllo e monitoraggio, qualità, performance.

Contributi: DC, revisione critica del lavoro; DC, EF, raccolta e analisi dei dati; EF, stesura del manoscritto.

Articolo pubblicato secondo la Creative Commons Attribution NonCommercial 3.0 License (CC BY-NC 3.0).

CCopyright E. Foglia e D. Croce, 2014

Licensee PAGEPress, Italy

QUADERNI - Italian Journal of Medicine 2014; 2:106-113 mente ineccepibili, assistendo in molti casi a una managerializzazione per legge e non per scelta o vocazione, ${ }^{2}$ il dilagare della strega è stato contenuto e l'attività assoggettata a un maggiore controllo. Possiamo dunque apporre il cartello THE END alla conclusione di questa narrazione? Non è assolutamente così.

$\mathrm{Nel} 2014$, passati molti anni da questi avvenimenti, è d'uopo volgersi al proprio passato di storie e miti, per ri-analizzarlo criticamente e comprendere come esso abbia potuto influire sul corso invitabile degli eventi e come potrà ulteriormente farlo nel futuro, al fine di trovare nuove soluzioni per la Sanità moderna.

La Legge 517 del $1993^{3}$ e le sue successive modifiche hanno portato il Sistema, gradualmente, a una maggiore focalizzazione verso i concetti di aziendalizzazione e regionalizzazione e verso un'attenzione marcatamente forzata ai criteri di finanziamento e accreditamento, con la generazione di una significativa quantità di flussi informativi aziendali, volti al monitoraggio degli output prodotti dagli enti erogatori.

È opinione comune e diffusa che sia stato proprio l'incipit fornito da questa norma a creare una pressante, ben strutturata e ramificata misurazione di una particolare dimensione valutativa, propria del percorso e/o del processo sanitario: l'output.

La produttività ha, così, acquisito una sempre più rilevante importanza all'interno del contesto sanitario, anche in funzione della relativa semplicità di misurazione e della necessità di ampliamento dell'offerta agli 
utenti, incentrando così su di essa le attenzione di tutti: dai Direttori Generali (per la loro esigenza di possedere un chiaro quadro di riferimento nell'ottica di una corretta contrattazione con Regioni ed enti regolatori), ai Direttori di Unità Struttura Complessa (per la necessità di governare le attività nell'ottica del raggiungimento degli obiettivi a loro attribuiti), fino al paziente (che sempre desidera conoscere informazioni circa il locus nel quale poter ricevere una determinata prestazione).

Il governo e la programmazione dell'attività sanitaria è senza ombra di dubbio un elemento imprescindibile: senza un'adeguata e ben strutturata negoziazione delle attività e prestazioni erogabili al cittadino, sussiste un elevato rischio di inappropriatezza, con un sistematico riflesso sugli aspetti economici e, quindi, di spesa.

Il monitoraggio della produzione sanitaria non può essere esclusivamente demandato alle aziende. Esso deve necessariamente rappresentare il punto di incontro tra Unità Strutture Complesse/Aziende Ospedaliere/ Presidi e le Aziende Sanitarie Locali con le Regioni, affinché tutti seguano le linee di indirizzo contenute nella programmazione strategica dell'attività.

In un contesto di devolution e decentralizzazione marcata, come quello da noi vissuto, però, le modalità tecniche di costruzione dei flussi di informazioni e gli strumenti di monitoraggio messi in atto differiscono sensibilmente, fino ad arrivare, in alcuni casi, a una completa impossibilità di paragonare le informazioni a disposizione, con elevati livelli di asimmetria informativa. Spesso i dati generati dai soggetti erogatori, non sono sempre i medesimi con i quali gli enti regolatori si trovano a dover giudicare l'attività, e nella sua complessità, queste discrepanze non sono sistematicamente comunicate e corrette, perpetuando comportamenti o convinzioni non completamente coerenti, con una eccessiva focalizzazione alla prestazione e non al suo risultato in termini di modifica dello stato di salute.

Questi elementi pongono le basi di una annosa questione intrinseca al sistema: non è più pensabile governare la complessità del Servizio Sanitario Nazionale esclusivamente con gli strumenti imposti dalla 502 del 1992, ma diviene assolutamente prioritario, per un migliore controllo, un'evoluzione verso approcci innovativi e soprattutto multidimensionali e multidisciplinari, che possano garantire il completamento del ciclo di misurazione e valutazione delle performance, chiudendo con modalità e tempistiche corrette l'anello di retroazione rappresentato dal processo di programmazione e controllo, così da avere un impatto sui risultati del servizio sanitario.

Non si vuole, con ciò, dire che gli esiti di breve e di medio periodo della norma siano stati fallimentari, anzi: se oggi, nel settore sanitario, il disavanzo è in una situazione di sostanziale controllo, è soprattutto merito di questa norma e delle sue successive integrazioni.

Finalità del nostro servizio sanitario di tipo univer- salistico è però quella di garantire salute a tutta la popolazione, erogando prestazioni di qualità. Come conciliare, dunque, le esigenze di carattere qualitativo e tecnico, con i bilanci malridotti del nostro Governo? Come agire dunque a questo proposito e quali strumenti e accorgimenti mettere in atto per un ulteriore miglioramento del sistema nel suo complesso?

Obiettivo del presente scritto è quello di indagare questa annosa questione, sotto un profilo tipicamente gestionale, con particolare attenzione alla Medicina Interna, area che acquisirà un sempre maggiore rilievo e interesse per $\mathrm{i}$ cambiamenti in atto in riferimento alla popolazione curata, a causa del generale invecchiamento della stessa. ${ }^{4}$ Per raggiungere questo obiettivo, si cercherà di comprendere e proporre le linee di sviluppo future da prendere in considerazione per poter garantire l'applicazione di strumenti che consentano tanto il monitoraggio delle variabili economiche, così di moda oggi, quanto, soprattutto, di quelle di efficacia, in un trade off virtuoso che possa portare al reale governo del sistema, nel suo complesso, indagando l'esistenza di modelli e di indicatori da poter applicare nel contesto di riferimento, ma adeguati alle esigenze di sistema.

Utilizzando una metafora, forse troppo fiabesca, ma molto calzante: nel regno di Sanità, nel Castello incantato della Medicina Interna, la principessa Qualità si trova prigioniera nella torre più alta e di difficile accesso. A fare da guardia due degli antagonisti più temibili di sempre: la strega Spesa e il mostro (nato dalla sua magica bacchetta) Taglio Lineare. Come potrà il principe Governo Clinico salvare la principessa e riportarla a essere protagonista indiscussa e governatrice giusta del regno di Sanità? Quali gli stratagemmi e gli strumenti da mettere in atto per portare a compimento questo salvataggio regale?

\section{Background}

Ipotesi di fondo è che tutti i sistemi di misurazione, monitoraggio e controllo siano utili: il problema è insito nel governo, nella gestione e nella scelta di questi, con particolare riferimento al sistema di rendicontazione italiano, che ha dettato le condizioni di raccolta dati sin dalla sua nascita ed evoluzione verso il pagamento a prestazione.

Una proposta di superamento dei più tradizionali sistemi di programmazione e controllo viene proprio dalla clinical governance (CG) ${ }^{5}$

Gli anglosassoni hanno teorizzato questo termine ed esso è ormai divenuto parte integrante delle nostre esistenze: tutti gli operatori sono convinti che il governo clinico abbia ampi potenziali, con il fine di portare a un miglioramento dei processi sanitari e, a cascata, dell'intero sistema. Ma come utilizzarlo e quali strumenti poter applicare?

Evidenze scientifiche ed empiriche ${ }^{6,7}$ sui buoni 
esiti generati dall'implementazione dei principi del governo clinico, hanno dimostrato la sua importanza e la rilevanza di temi quali la multidisciplinarità e multidimensionalità.

Ma perché allora, nonostante convinzioni e manifesti, vi è ancora uno scarso recepimento di questi principi nella pratica di tutti i giorni? $?^{8,9}$

Indubbiamente l'attuazione della clinical governance genera, in primis, un problema di ordine comportamentale e relazionale; coloro i quali conoscono tecnicamente meglio gli strumenti di CG sono i clinici, che non possono però pensare di detenere lo scettro del potere, senza agire in ottica, appunto, multidisciplinare. Affinché la CG porti i frutti sperati, e sia essa stessa strumento non solo di misurazione, ma anche,di correzione delle pratiche non adeguate, deve attivare un percorso di coinvolgimento verticale del livello manageriale ${ }^{10}$ e di costruzione di una leadership diffusa, che possa agevolmente favorire l'integrazione di questa modalità di lavoro con i livelli operativi e preordinati esistenti, ${ }^{9}$ anche in termini informativi e culturali, così da essere effettivamente a vantaggio di tutte le categorie professionali operanti in Sanità.

In questo modo, sarebbe realmente possibile perseguire, attraverso il raggiungimento e il controllo della qualità, anche obiettivi di tipo economico, realizzando due goal importantissimi, attraverso uno strumento univoco: il ciclo di misurazione e di valutazione si chiuderebbe in un contesto di eccellenza clinica.

La condivisione tra management e clinica porterebbe a una focalizzazione sul concetto di qualità delle prestazioni e non solo sul numero delle stesse. L'inquadramento di tale tematica ci porta a evidenziare come esso non sia un termine univoco, bensì polisemico, e di per sé multidimensionale, esattamente come avviene per la CG che viene considerata un ombrello, al di sotto del quale possono essere nascoste sfaccettature e considerazioni di differente tipologia. ${ }^{11,12}$ Perseguire la qualità delle prestazioni in un'ottica di appropriatezza garantisce anche il miglior rapporto economico della cura, senza sprechi. . $^{13,14}$

A tal proposito alcuni autori fanno comprendere come, partendo dalla definizione stessa di qualità, essa rappresenti un termine dai differenti e significativi impatti, almeno su quattro fronti differenti: la qualità tecnica e delle conoscenze, quella organizzativa e delle circostanze, la percepita dall'utenza e, infine, quella economica, relativa alle risorse a disposizione. ${ }^{15}$ Tale concetto può essere ulteriormente declinato, considerando altri elementi insiti nella qualità quali: efficacia teorica (efficacy), efficacia pratica (effectiveness), appropriatezza, competenza, efficienza, accessibilità e livello di soddisfazione. È possibile, però, ritrovare in tutte queste dimensioni, una matrice comune e sintetizzata, riferibile ai succitati impatti. ${ }^{16} \mathrm{~A}$ fronte di tali definizioni, diviene chiaro come, per monitorare la qualità nel suo complesso, sia indispensabile misurare due principali categorie di interazioni: quelle esistenti tra queste dimensioni e gli esiti delle prestazioni, e quelle che intersecano tra loro le differenti dimensioni.

Il monitoraggio della qualità clinica, infatti, non si discosta dalla efficienza economica, sono due facce della medesima medaglia. Il perseguimento della qualità porta a una maggiore appropriatezza, che, a sua volta, genera un risparmio complessivo, il quale può essere raggiunto con l'eliminazione di sprechi e inefficienze e con la revisione dei processi sanitari, grazie alla quale questi diverranno più snelli. Se l'implementazione di sistemi di qualità risulta essere, invece, vettore di maggiori costi, significa di per certo che essa è inappropriata o implementata nella modalità non corretta; è, pertanto, da evitare, non principalmente in un'ottica gestionale, ma soprattutto per il bene dell'utilizzatore finale, il paziente. ${ }^{17}$

Ultima considerazione è riferibile all'esigenza di localizzare tutte queste considerazioni rispetto alle risorse e al tempo. Differente sarà misurare la produttività in una situazione con a disposizione molti o pochi input (molte o poche risorse), come differente sarà cercare informazioni sugli esiti clinici (outcome) e non sugli output. È possibile in un breve periodo reperire informazioni per la valutazione della produttività, ma saranno necessari anche diversi anni per avere un riscontro derivante dalla misurazione del ritorno di salute di un intervento sanitario. ${ }^{18}$ In breve: per la misurazione dei risultati di salute e della qualità in termini di efficacia saranno necessari più anni, e, quindi, un orizzonte temporale che, in chiave economica, sarebbe pluriennale e pertanto misurabile in più cicli di programmazione e controllo.

Concorrono a complicare il quadro, ovviamente, le diverse esigenze delle specificità cliniche, per la misurazione degli outcome.

La metodologia può essere condivisa, ma le informazioni sintetiche reperite devono essere di rilievo e di facile lettura, sia per il clinico, sia per il gestore, salvaguardando le richieste strategiche di ogni ente erogatore, nonché la particolarità clinica e la relativa localizzazione.

Sarà possibile, dunque, strutturare un impianto di tipo multidimensionale e multiprofessionale, anche al riparo metodologico della clinical governance, ma con obiettivi differenti; le informazioni da utilizzare per alimentare tecnicamente questo modello dovranno essere diverse e pertanto anche gli indicatori per misurare il raggiungimento degli obiettivi saranno necessariamente differenti.

Molte sono le definizioni in letteratura su indicatore o set di indicatori. Casati definisce indicatore una grandezza sintetica che rappresenti un fenomeno e che si presti a dare giudizi, comprendendo al suo interno i fattori che hanno determinato il fenomeno stesso. ${ }^{19}$ 
L'esistenza di una pluralità di definizioni di indicatori è espressiva della complessità del tema e ciò porta a ribadire come la scelta degli stessi debba essere attentamente correlata all'obiettivo di valutazione e all'oggetto di misurazione. In avvio deve essere chiaro l'obiettivo da cogliere, nella sequenza logica: i) definizione dell'obiettivo che si vuole cogliere; ii) individuazione dell'indicatore; iii) determinazione del target (dove poniamo l'asticella).

È essenziale, dunque, non limitarsi alla costruzione di un insieme di indicatori, ma, una volta specificato il concetto multidimensionale di qualità che si vuole valutare, è opportuno arrivare a costruire un sistema di misurazione delle performance, in grado di monitorare tutto il ciclo, dalla programmazione alla valutazione.

\section{Sistemi di misurazione delle performance}

Nel nostro Paese i sistemi di misurazione e monitoraggio sono a uno stadio di sviluppo piuttosto embrionale $^{20}$ e si assumono decisioni con modalità qualitative e non sempre oggettive. ${ }^{21}$

A far breccia in questa situazione tutt'altro che positiva è stato il D. Lgs. 150 del 2009, ${ }^{22}$ conosciuto come Legge Brunetta, che ha dato uno scossone alla Pubblica Amministrazione nazionale, parlando, per la prima volta, di performance del sistema, suddividendola tra organizzativa e individuale. Sicuramente la spinta a una innovazione del genere è stata di tipo top down: il recepimento di una normativa europea, quale la Tallin Charter, ${ }^{23}$ ha fornito uno slancio e uno spunto allo sviluppo di nuove indicazioni per il governo del sistema.

Il linguaggio della PA è cambiato repentinamente, strutturando degli articolati piani di misurazione della performance, che prendano in considerazione sia la sua ampiezza sia la sua profondità. Questa situazione ha portato sui tavoli dei manager sanitari e dei decisori di spesa la necessità di cercare un collegamento tra esigenze di budget e di qualità: il problema risiede nel fatto che, data la complessità di questo percorso, molti si sono fermati alla ricerca del collegamento, e si sono persi in questa fase di implementazione di sistemi di misurazione e valutazione della performance. ${ }^{24}$

Esattamente come è avvenuto per la definizione di qualità e di indicatore, anche per il termine performance la letteratura fornisce diverse accezioni e non esiste un accordo univoco sullo specifico contesto semantico di riferimento.

Gli autori concordano, però, nel sottolineare come la performance possa abbracciare aspetti di efficienza ed efficacia, ${ }^{25}$ che, attraverso la sua misurazione, dia la possibilità alle organizzazioni di progredire, fornendo una fotografia e uno spaccato della situazione attuale, in comparazione con quella futura, passando per l'oggettivazione del raggiungimento o del non raggiungimento di specifici obiettivi. ${ }^{26}$
Un sistema di misurazione delle performance, però non deve essere solamente focalizzato sulla definizione di quale performance e, quindi, risultato, debba essere preso in considerazione, ma necessita anche di specifiche caratteristiche. Il processo di quantificazione deve essere continuo, alimentato da flussi possibilmente esistenti, facilmente reperibili e non periodici; la sua raccolta dati deve essere specifica rispetto alle esigenze di misurazione degli obiettivi prescelti, deve prediligere una lettura e una validità interna all'azienda, per la sua natura di strumento di controllo e monitoraggio interno, e non di valutazione esterna; la ritualità di definizione degli obiettivi e delle finalità di misurazione deve essere comunicata in anticipo ai soggetti che comparteciperanno al raggiungimento degli obiettivi aziendali, e che saranno, poi, valutati rispetto alle attività svolte e ai comportamenti tenuti. ${ }^{24,27}$

È rilevante poi che si sia in grado di cogliere gli aspetti di ampiezza e di profondità della performance: non solo essa deve trovare una istituzionalizzazione all'interno dei diversi ruoli organizzativi ed essere agganciata al responsabile dell'obiettivo e del raggiungimento di quest'ultimo nel contesto lavorativo di riferimento, ma deve poter agganciare tutte le dimensioni che prima erano state definite come di input, output, outcome, processo, fino a cogliere l'impatto che essa ha sull'utilizzatore finale e quindi sul paziente. ${ }^{24,27}$

Un buon sistema di misurazione della performance, oltre a definire coerentemente gli obiettivi, dovrebbe riuscire a porli in essere in modo tale da percorrere orizzontalmente e verticalmente tutti i substrati dell'organizzazione e tutta l'ampiezza della performance. Solo con questa modalità sarà possibile garantire un monitoraggio coerente e completo delle prestazioni erogate e del ritorno di salute.

All'interno di questo contesto, variegati sono i modelli che hanno cercato di riportare tali caratteristiche di massima, cogliendo la multidimensionalità insita all'interno della qualità e delle performance, declinando differenti indicatori che andassero a misurare molteplici sfaccettature: dalla famosissima Balanced Scorecard, ${ }^{28}$ al nazionale bersaglio della Regione Toscana, ${ }^{29}$ passando, ovviamente, per la clinical governance.

All'estero i sistemi di misurazione delle performance in sanità (intesa come precedentemente definita) si sono sviluppati nel Regno Unito, in USA e in Francia come pay for performace (P4P). In UK gli incentivi negativi alla qualità sono stati avviati con una iniziativa nel 2004, conosciuta come quality and outcomes framework (QOF) [a questo riguardo, ulteriori informazioni sono disponibili sul sito: http://www.hscic.gov.uk/ services/qof consultato in data 8 agosto 2014]. Negli Stati Uniti le maggiori esperienze riguardano Medicare e le sue iniziative per incentivare i clinici e gli ospedali a cercare di migliorare la qualità delle cure ed evitare costi sanitari non necessari. Tutti questi sistemi ricom- 
prendono indicatori come durata di degenza, ricoveri inappropriati, mortalità intraospedaliera e tasso di rericovero a 30 giorni, ai quali aggiungono sempre la $\mathrm{cu}$ stomer satisfaction e le infezioni nosocomiali.

Altri indicatori più sofisticati si possono vedere nella Tabella 1.

I sistemi di misura pay-for-performance basati su misurazioni di indicatori clinici specifici hanno alcune controindicazioni. La prima è che in alcuni servizi sanitari i provider hanno un forte incentivo a deselezionare (rifiutare di accettare) i pazienti che hanno probabilità di esito sotto lo standard di qualità richiesto (si pensi ai pazienti non complianti). La seconda è che i migliori reparti tendono ad attrarre i pazienti più complicati e quindi i loro outcome possono risultare inferiori a reparti con effettiva minore esperienza. La terza è che in una rosa di pochi indicatori i provider tenderebbero a seguire i risultati di questi e non lo stato di salute complessivo del paziente (senza seguire, in pratica, le esigenze della presa in carico).

Per ovviare a questi difetti si tende a utilizzare più categorie di indicatori come la classifica US News and World Report degli ospedali. Questa si basa su: i) reputazione della struttura; ii) elementi strutturali (accreditamento); iii) outcome; iv) soddisfazione del paziente; e v) sicurezza. Il peso assegnato a ciascuna area cambia, così come il numero di componenti delle aree.

\section{Discussione}

Pur avendo vissuto una fase di nuovo fermento, sussistono diverse problematiche e il processo di miglioramento non può dirsi concluso, almeno non per tutte le realtà che operano nel contesto sanitario.

Ciò è dovuto, prima di tutto, alla devolution, che ha consentito il fiorire di modelli di valutazione delle performance differenti, tendenzialmente sviluppati su base regionale. Se ciò garantisce un maggior adattamento, con una alimentazione delle informazioni su base locale, dall'altra parte non consente un confronto di tipo nazionale, con il risultato ultimo di una complessiva impossibilità di paragonare le differenti realtà produttive. Questo potrebbe sembrare un controsenso date le numerose attività di benchmarking a livello nazionale che hanno visto come oggetto di indagine le performance dei sistemi regionali.

Nonostante l'impianto di tipo multidimensionale e multidisciplinare, infatti, gli esempi a oggi esistenti, anche utilizzati per comparazioni interregionali, non sono realmente volti al tentativo di cogliere tutta l'ampiezza della performance, nonché la sua profondità da un punto di vista organizzativo. Il fatto che questi rispondano alle esigenze dettate dalla normativa e dalla letteratura non può essere, di per sé, una condizione sufficiente a garantire l'efficacia del sistema di controllo, e la misurazione della qualità dei processi.

L'incapacità di questi sistemi di cogliere l'essenza dell'attività condotta genera delle problematiche significative: strutture ospedaliere a bassa complessità, nei ranking nazionali, risultano essere i best performer, non perché non lo siano veramente, ma semplicemente per il fatto che gli indicatori costruiti non sono in grado di cogliere elementi discriminanti quali l'attrattività dell'ospedale, il grado di complessità e la reputazione dello stesso.

Tabella 1. Condizioni del modello pay for performance di Medicare (USA).

Obiettivi per le strutture ospedaliere rimborsate secondo il metodo pay for performance

La misurazione dei seguenti obiettivi è essenziale per le strutture ospedaliere che presentano le performance distribuite nei quartili migliori, soggette a penalità finanziarie, secondo il risk-adjustment.

A queste strutture in caso di performance negative (come distribuzione rispetto ai percentili) è dedotto 1'1\% del rimborso DRG (aggiornato fino al 2015).

Le 10 categorie di attività all'interno delle quali vengono definiti gli obiettivi sono:

1. Presenza di corpi estranei in sede, post intervento chirurgico

2. Embolismo gassoso provocato

3. Trasfusioni e incompatibilità del gruppo sanguigno

4. Presenza di lesioni da pressione di terzo e quarto stadio della scala NPUAP

5. Cadute e traumi

6. Manifestazioni cliniche legate a uno scarso controllo del livello di glucosio

7. Infezioni urinarie catetere correlate

8. Infezioni vascolari catetere correlate

9. Infezioni del sito chirurgico a seguito di:

- Bypass coronarico

- Chirurgia bariatrica

- Intervento ortopedico

10. Tromboembolismo venoso o embolia polmonare 
Si tratta, quindi, di sistemi di misurazione, che, pur essendo multidisciplinari, governano con indicatori utili, ma generali e tendenzialmente basati su flussi di produttività e di monitoraggio dei soli processi. Non è sicuramente pensabile poter essere efficaci, senza il possesso e la divulgazione di importanti informazioni quali la degenza media, il tasso di saturazione, la percentuale di diagnosis-related group (DRG) medici provenienti da reparti chirurgici, la percentuale di ricoveri medici ripetuti entro i 30 giorni, il tasso di mortalità intraospedaliero, ecc... Ma quale riflesso immediato sulla qualità dei processi sanitari possono avere questi indicatori?

L'esperienza internazionale ci indica come debbano sussistere delle metodiche di correzione o categorizzazione dei risultati derivanti dagli indicatori, rispetto a complessità, qualità dei risultati o almeno qualità percepita dall'utenza, altrimenti tutte le classifiche e i confronti, anziché generare valore aggiunto, non produrranno altro se non indifferenza o sospetto, così come avviene in Italia, oggi. Questo non è un problema legato alla non validità dei sistemi di valutazione delle performance, ma da come questi vengono posti in essere: prima è necessario considerare il livello qualitativo generale, trovato un modo per comprendere ciò e la complessità clinica e assistenziale della casistica trattata, solo poi, sarà possibile costruire degli indicatori di sintesi, anche gestionali, per il confronto di input utilizzati, output prodotti e outcome finali.

Per migliorare i percorsi e i processi all'interno del contesto della Medicina Interna, è sufficiente monitorare i DRG per LEA medici e il tasso di ospedalizzazione per il diabete? Essi sono utili per far comprendere il posizionamento rispetto alla popolazione complessiva e sono sicuramente adeguati per simulare un mercato di prestazioni omogenee, ma ne misurano realmente la qualità? Forniscono un feedback sul grado di miglioramento del singolo percorso? Sono pesati rispetto all'attrattività e reputazione della specifica azienda?

Se si ripercorrono questi modelli, rievocando la necessità di engagement tra clinici e tecnici, tanto richiesta dalla clinical governance, si arriva alla conclusione che è ancora necessario lavorare approfonditamente su questo tema.

È, quindi, opportuno andare oltre al già tracciato, per non agire nell'ottica di riciclare l'esistente seppur con altre finalità, ma per strutturare delle nuove proposte, per costruire nuovi meccanismi e soprattutto nuovi flussi informativi, che possano alimentare nuovi indicatori. Come cogliere, quindi, tutte le sfaccettature di tale elemento di valutazione?

Per fare ciò è necessario costruire e strutturare gli obiettivi in modo molto attento, definendo, innanzi tutto, il livello di profondità al quale fanno riferimento, sotto un profilo organizzativo o individuale, struttu- rando l'obiettivo in base alle richieste di pianificazione strategica, per poi passare alla declinazione degli indicatori, al razionale, quindi, alla loro spiegazione e formula di calcolo, così che sia chiaro a tutti come l'indicatore venga numericamente costruito. Infine, è fornito un target di raggiungimento, per dichiarare il grado di compliance dell'individuo e dell'organizzazione, nei confronti di quell'obiettivo prefissato, nel breve e nel lungo periodo.

È vero che agendo sulle attività e sulle modalità di svolgimento delle prestazioni si ha la possibilità di incidere sulle performance e sul loro ottenimento, sia a livello di componenti dell'organizzazione sia di enti in senso più ampio, facendo convergere l'azione individuale e quella organizzativa; ${ }^{30}$ in questo modo si è, però, esclusivamente incentrati su una logica di processo.

È proprio questo il meccanismo da scardinare, ossia il fatto che un sistema di misurazione sia solo incentrato sul processo e non sulla qualità finale, facendo remare, ogni singolo attore, verso rotte differenti... ma come fermare tale migrazione?

\section{Conclusioni}

L'adozione di adeguati sistemi informativi per la programmazione e il controllo dovrebbe consentire di valutare la rispondenza delle attività e delle prestazioni erogate rispetto alle attese dell'utenza, nel rispetto dell'allocazione adeguata di risorse e in coerenza con la struttura organizzativa, perseguendo principi di qualità.

L'adeguamento di questi sistemi alla visione strategica e l'orientamento al risultato sono le strade da percorrere per intraprendere un vero e proprio cambiamento all'interno dei sistemi ove l'Amministrazione Pubblica presenta ancora difficoltà e problematiche organizzative e di risorse, così come avviene nel nostro paese. ${ }^{31}$

Per introdurre una cultura di orientamento al risultato è necessario attivare un monitoraggio che non sia solo per processi, ma che sia rivolto a tutte le dimensioni della performance; questo può essere garantito solo con una maggiore attenzione verso le modalità di strutturazione degli obiettivi, condividendo la stessa tra componente clinica e manageriale, e imponendo l'applicazione di obiettivi che riguardano l'outcome.

Vi è quindi una necessità di superamento dei flussi informativi ad oggi esistenti e una esigenza di strutturarne di nuovi. Chiave di volta perché questo avvenga, è, nuovamente, il coordinamento di tutte le figure professionali, ciascuna rispetto alle proprie competenze, seguendo le esigenze specifiche per singola componente dell'organizzazione e per singola Unità Operativa.

Il mondo scientifico concorda con questa esigenza, trovando una totale condivisione di intenti tra pianeta 
del sapere e satellite del fare: tantissimo però può e deve ancora essere fatto.

La value-based medicine, ${ }^{32}$ l'iniziativa internazionale di choosing wisely, ${ }^{33}$ unitamente a diverse iniziative nazionali che stanno cercando di veicolare la cultura del risultato e delle scelte basate sull'appropriatezza e quindi sul corretto uso delle risorse, potranno sicuramente fornire una motivazione per modificare l'attuale situazione e incentivare un cambiamento profondo. È importante notare come movimenti di questa portata per primi, non solo vogliono basare il fare sanitario sul sapere, sulla qualità e sulla appropriatezza, ma mostrano come, la maggiore appropriatezza porti a una riallocazione più adeguata delle risorse e a una reale possibilità di monitorare le attività sanitarie, dunque anche le quantità prodotte, arrivando all'importante concetto che chi maggiormente produce con qualità, dovrebbe sostituirsi a chi meno produce e con risultati scadenti.

È necessario portare queste scuole di pensiero anche all'interno del mondo della Medicina Interna, con una maggiore focalizzazione su appropriatezza e qualità, in modo tale che gli obiettivi ipotizzati, in coerenza con il sistema, siano concordati anche su scala nazionale: ottimale sarebbe la veicolazione degli stessi grazie al supporto delle società scientifiche. È importante che vi sia un coordinamento e un accordo da un punto di vista tecnico su quelle che sono le performance specifiche da misurare, che oggi non trovano una indicazione nel sistema.

La gestione e il governo della Sanità, senza la misurazione e il possesso delle informazioni è impensabile. Risulta, infatti, di difficile comprensione la separazione del concetto di misurazione da quello di management: sintetizzare una performance attraverso un obiettivo e un indicatore, senza pensare, contemporaneamente, a come gestire il risultato che la misurazione consegnerà, è impossibile. ${ }^{34}$ Questo vale per qualsiasi tipo di performance.

\section{Bibliografia}

1. Decreto Legislativo n. 502 1992, Riordino della disciplina in materia sanitaria, a norma dell'art. 1 della Legge 23 Ottobre 1992, n.421.

2. Marasca S. La managerializzazione della p.a. richiede "la forma aziendale"? In: Forte F, Robotti L. (a cura di) La gestione manageriale nella pubblica amministrazione. Milano: FrancoAngeli; 2006.

3. Decreto Legislativo n. 517 1993, Modificazioni al Decreto Legislativo 30 Dicembre 1992, n. 502, recante riordino della disciplina in materia sanitaria, a norma dell'art. 1 della Legge 23 Ottobre 1992, n. 421.

4. Stevens A, Milne R, Lilford R, Gabbay J. How do new technologies get into practice? Keeping pace with new technologies: systems needed to identify and evaluate them, BMJ 1999;319:1291-4.
5. Scally G, Donaldson L. Clinical governance and the drive for quality improvement in the new NHS in England. BMJ 1998;317:61-5.

6. Ravaghi H, Zarnaq RK, Adel A, et al. A survey on clinical governance awareness among clinical staff: a crosssectional study. Global J Health Sci 2014;6.

7. Specchia ML, La Torre G, Siliquini R, et al. OPTIGOV - A new methodology for evaluating Clinical Governance implementation by health providers. BMC Health Serv Res 2010;10:174-89.

8. deBurca S, Williams P, Armstrong C. The pursuit of quality: a clinical directorates' progress in clinical governance. A case study of the Women and Children's Directorate, Galway University Hospital. Limerick: Health Systems Research Centre and Galway University Hospital; 2008.

9. Gauld R, Horsburgh S, Brown J. The clinical governance development index: results from a New Zealand study. BMJ Qual Saf 2011;20:947-53.

10. Ravaghi H, Heidarpour P, Mohseni M, Rafiei S. Senior manager's viewpoints toward challenges of implementing clinical governance: a national study in Iran. Int J Health Policy Manage 2013;1:295-9.

11. Braithwaite J, Travaglia J. An overview of clinical governance policies, practices and initiatives. Austr Health Rev 2008;32:10-22.

12. McSherry R, Pearce P. Clinical governance: a guide to implementation for healthcare professionals. 3rd ed. Oxford: Wiley Blackwell Publishing Ltd.; 2011.

13. Grilli R, Taroni F. Governo clinico. Roma: Il pensiero scientifico editore; 2004.

14. Wright J, Hill P. La governance clinica. Milano: McGraw-Hill; 2005.

15. Donabedian A. La qualità dell'assistenza sanitaria. Roma: Nuova Italia Scientifica; 1990.

16. Focarile F. Indicatori di qualità nell'assistenza sanitaria. Torino: Centro Scientifico Editore; 1998.

17. Walsh K. Clinical governance: costs and benefits. Int J Health Policy Manage 2014;2:149.

18. Vaccani R. La sanità incompiuta. Roma: La Nuova Italia Scientifica; 1991.

19. Casati G. Programmazione e controllo di gestione nelle aziende sanitarie pubbliche. Milano: Mc Graw-Hill; 2000.

20. Boland T, Fowler A. A systems perspective on performance management in public sector organizations. Int $\mathrm{J}$ Public Sector Manage 2000;13:417-46.

21. Riccaboni A. Il controllo di gestione. Milano: McGrawHill; 2001.

22. D. Lgs 150/2009, Ottimizzazione della produttività del lavoro pubblico, efficienza e trasparenza delle PA. Gazzetta Ufficiale 31 ottobre 2009.

23. Resolution EUR/RC58/R4. The Tallin Charter: Health System for Health and Wealth; 2008.

24. Neely A, Mills J, Platts K, et al. Performance measurement system design: developing and testing a processbased approach. Int $\mathrm{J}$ Operat Prod Manage 2000;20:1119-45.

25. Neely A, Gregory M, Platts K. Performance measurement system design - a literature review and research agenda. Int J Operat Prod Manage 1995;15:80-116.

26. Rose KH. A performance measurement model. Qual Progr 1995:63-6.

27. Neely A. The performance measurement revolution: 
Why now and what next? Int J Operat Prod Manage 1999;19:205-28.

28. Kaplan RS, Norton DP. Transforming the balanced scorecard from performance measurement to strategic management: Part II. Account Horiz 2001;15:147-60

29. Nuti S. La valutazione della sanità Toscana: Report 2006. Pisa: Edizione ETS; 2007.

30. Rebora G. La valutazione dei risultati nelle amministrazioni pubbliche. Milano: Guerini e Associati; 1999.

31. Osborne D. Reinventing government: What a difference a strategy makes. $7^{\circ}$ Global Forum on Reinventing Government. Vienna: Building Trust in Government; 2007.

32. Porter M, Teisberg EO. Redefining health care creating value-based competition on results. Harvard: Harvard Business School Press; 2006.

33. ABIM Foundation. Choosing wisely. Disponibile a: www.choosingwisely.org Consultato in data: 20 Luglio 2014.

34. Lebas MJ. Performance measurement and performance management. Int J Prod Econ 1995;41:23-35. 


\title{
Medicina Interna e rischio clinico: criticità e possibili correttivi
}

\author{
Giuseppe Chesi, ${ }^{1}$ Filippo Dall'Orto, ${ }^{1}$ Pietro Ragni, ${ }^{2}$ Roberto Nardi ${ }^{3}$ \\ ${ }^{1}$ UOC Medicina Interna, Ospedale C. Magati, Scandiano AUSL di Reggio Emilia; ${ }^{2}$ Direzione Sanitaria, Area Rischio Clinico, \\ AUSL di Reggio Emilia; ${ }^{3}$ Medicina Interna, Bologna, Italia
}

\section{RIASSUNTO}

Negli ultimi anni il rischio clinico (la probabilità che un paziente subisca un evento avverso imputabile agli interventi clinici o assistenziali) è stato particolarmente enfatizzato in relazione all'aumentare dei contenziosi in ambito sanitario, con processi e dinamiche che sempre più tendono ad avvicinarsi al modello nord-americano. La tipologia dei pazienti ricoverati nei reparti di Medicina Interna richiede un cambiamento di mentalità e di approccio ma anche un cambiamento nella definizione delle strategie organizzative e gestionali per la gestione non solo della fase acuta. Gli aspetti che maggiormente risultano correlati al rischio clinico in Medicina Interna riguardano prevalentemente: i) i percorsi di ammissione e dimissione che coinvolgono l'ospedale in toto (a partire dal pronto soccorso) ma anche il territorio (medici di famiglia e Servizi Sociali); ii) i percorsi clinico-assistenziali per le patologie più frequenti (al fine di uniformare i comportamenti importando le indicazioni dell'evidence-based medicine nella realtà in cui si opera; iii) la corretta compilazione della documentazione sanitaria (finalizzata a riflettere, in modo sintetico ma esaustivo, la qualità dell'approccio assistenziale ad ogni specifico problema e molto utile sia come strumento di audit che per la tutela medicolegale; iv) le modalità di prescrizione e somministrazione della terapia (sia in ambito di appropriatezza che nell'ambito di prescrizione, preparazione e somministrazione, ambiti questi ultimi nei quali gli strumenti elettronici si sono dimostrati in grado di limitare il rischio di errori); v) l'applicazione delle più adeguate misure igienico sanitarie nella gestione e nel trattamento dei pazienti (fondamentali per ridurre l'incidenza di infezioni ospedaliere); vi) adeguatezza della dotazione organica in riferimento ai carichi di lavoro (non solo in relazione alla numerosità del personale in servizio ma anche alla sua distribuzione; fondamentale infatti è l'omogenea distribuzione delle competenze al fine di uniformare il rendimento della struttura sviluppabili anche attraverso mirati programmi di formazione continua del personale in servizio). Partendo dai dati della letteratura e dall'esperienza sul campo, gli autori analizzano sinteticamente i singoli fattori e le possibili azioni di miglioramento ad essi collegate.

\section{Introduzione}

Negli ultimi anni il concetto di rischio clinico è entrato tra le dimensioni da considerare nell'ambito ospedaliero, al pari di quello di organizzazione, dell'utilizzo appropriato delle risorse e della qualità dell'assistenza. ${ }^{1,2}$ Il rischio clinico è stato particolarmente enfatizzato in relazione all'aumentare dei contenziosi in ambito sanitario, ${ }^{3}$ con processi e dinamiche che, seppure con una certa latenza, sempre più tendono ad avvicinarsi al modello nord-americano. Anche se tale ambito risulta relativamente nuovo, l'interesse per identificare e cercare correttivi per le problematiche ad esso connesse è stato

Corrispondente: Giuseppe Chesi, viale Martiri della Libertà 2, 42019 Scandiano (RE), Italia.

E-mail: chesig@ausl.re.it

Parole chiave: rischio clinico, medicina interna, appropriatezza, organizzazione sanitaria.

Articolo pubblicato secondo la Creative Commons Attribution NonCommercial 3.0 License (CC BY-NC 3.0).

CCopyright G. Chesi et al., 2014

Licensee PAGEPress, Italy

QUADERNI - Italian Journal of Medicine 2014; 2:114-124 notevole, particolarmente da parte dei Servizi di Medicina Legale delle aziende sanitarie.

La tipologia dei pazienti ricoverati nei reparti di Medicina Interna, costituita da persone anziane, affette da multiple malattie croniche coesistenti nello stesso individuo, spesso riacutizzate e con fasi critiche di instabilità clinica, necessita, oltre che di un cambiamento di mentalità e di approccio, anche di una diversa definizione delle strategie organizzative per la loro gestione, non limitata solamente alla fase acuta. Questi concetti sono stati oggetto di numerosi interventi nella letteratura degli ultimi anni., ${ }^{4,5}$

Secondo la definizione più classica, il rischio clinico esprime la probabilità che un paziente subisca un evento avverso, ovvero che riceva un qualsiasi danno o disagio imputabile, anche involontariamente, agli interventi clinici o assistenziali prestati durante il periodo di degenza o di assistenza, in grado di causare un prolungamento del periodo di ricovero, un peggioramento delle condizioni di salute o la morte. ${ }^{6}$ Charles Vincent ha ben chiarito che l'approccio alla sicurezza, ovvero alla gestione del rischio clinico, può avvenire secondo due visioni nettamente distinte per filosofia, considerate complementari. ${ }^{7}$ La prima, che lui identifica con l'espressione Rimpiazzare e supportare l'uomo, pone l'accento sull'errore e sulla fallibilità e propone interventi tecnici e procedurali come progettazione, standardizzazione, 
protocolli e linee guida, tecnologie di gestione. La seconda, I professionisti creano la sicurezza, fonda le sue basi sull'adattabilità allo stress, la capacità di previsione e la resilienza. In questo lavoro abbiamo privilegiato il primo approccio, pur in modo non esclusivo.

Gli aspetti che maggiormente risultano correlati al rischio clinico in Medicina Interna (ma non solo) riguardano prevalentemente (Tabella 1): i) i percorsi di ammissione e dimissione; ii) i percorsi clinico-assistenziali per le patologie più frequentemente causa di ricovero; iii) la corretta compilazione della documentazione sanitaria; iv) le modalità di prescrizione e somministrazione della terapia; v) l'applicazione delle più adeguate misure igienico sanitarie nella gestione e nel trattamento dei pazienti. Inoltre, solitamente più trascurati, ma di grande importanza soprattutto in medicina interna, sono l'adeguatezza della dotazione organica in riferimento ai carichi di lavoro secondo diversi periodi temporali, alla dotazione e disponibilità di posti letto, ai modelli organizzativi ed infine alla disponibilità ed accessibilità alle tecnologie necessarie.

Di seguito vengono analizzati, seppure in maniera sintetica, questi fattori, i possibili correttivi ipotizzabili, frutto di esperienze sul campo e dai dati della letteratura, questi ultimi spesso ancora parziali e frammentari.

Tabella 1. Ambiti del rischio clinico in Medicina Interna.

\begin{tabular}{|c|c|c|}
\hline Ambito & Principali tipologie di errori (guasti) & Possibili correttivi \\
\hline Percorsi di ammissione e dimissione & $\begin{array}{l}\text { - Eccessiva permanenza in pronto soccorso } \\
\text { - Allocazione in area non adeguata per } \\
\text { tipologie a dotazione di personale e } \\
\text { tecnologia presente per il tipo di patologia } \\
\text { o di instabilità clinica del paziente } \\
\text { - Dimissione non protetta } \\
\text { - Lettera di dimissione incompleta } \\
\text { - Non adeguata informazione ed educazione } \\
\text { per le terapie prescritte }\end{array}$ & $\begin{array}{l}\text { - Creazione di aree intermedie (admission } \\
\text { unit, osservazione breve, ecc.) } \\
\text { - Creazione di percorsi alternativi al } \\
\text { ricovero (ad esempio Day Service) } \\
\text { - Format predefinito di Lettera di } \\
\text { dimissione con campi obbligatori } \\
\text { - Strutturazione di percorsi ad hoc con } \\
\text { checklist definite per ammissione e } \\
\text { dimissione }\end{array}$ \\
\hline $\begin{array}{l}\text { Gestione delle patologie principali (per } \\
\text { numerosità) trattate dalla Unità Operativa }\end{array}$ & $\begin{array}{l}\text { Variabilità ed improvvisazione nella gestione } \\
\text { delle principali a causa di: } \\
\text { - Non adesione alle evidenze scientifiche } \\
\text { - Difficoltà nella continuità assistenziale } \\
\text { - Impossibilità di verificare i risultati }\end{array}$ & $\begin{array}{l}\text { - Percorsi clinico assistenziali per le } \\
\text { patologie più frequentemente ricoverate } \\
\text { con checklist dedicate } \\
\text { - Gruppi di lavoro per la gestione e } \\
\text { supervisione } \\
\text { - Audit dedicati per la verifica degli } \\
\text { indicatori di processo e di outcome } \\
\text { associati }\end{array}$ \\
\hline Documentazione sanitaria & $\begin{array}{l}\text { - Incompletezza di informazioni, in } \\
\text { qualunque passaggio } \\
\text { - Mancata segnalazione di patologie } \\
\text { significative } \\
\text { - Mancata segnalazione di allergie od } \\
\text { intolleranze } \\
\text { - Non compilazione od aggiornamento } \\
\text { dell'esame obiettivo } \\
\text { - Non motivazione di scelte diagnostiche o } \\
\text { terapeutiche } \\
\text { - Mancato aggiornamento del diario clinico } \\
\text { (sia da medici dell'UO che da consulenti) } \\
\text { - Scarsa leggibilità delle informazioni dati } \\
\text { trascritti } \\
\text { - Mancata evidenza di presa visione } \\
\text { tempestiva di esami bioumorali e/o } \\
\text { strumentali eseguiti } \\
\text { - Problemi connessi al software (difetti, } \\
\text { interruzioni, macchinosità) } \\
\text { - Violazione dei passaggi obbligati del } \\
\text { software }\end{array}$ & $\begin{array}{l}\text { - Cartella clinica informatizzata con sistemi } \\
\text { integrati di alert } \\
\text { - Percorsi strutturati di formazione } \\
\text { - Audit di verifica e miglioramento }\end{array}$ \\
\hline Informazione e condivisione con l'utenza & $\begin{array}{l}\text { - Non adeguatezza della comunicazione ed } \\
\text { informazione al paziente ed ai familiari in } \\
\text { merito all'iter diagnostico e terapeutico in } \\
\text { corso ed alla prognosi } \\
\text { - Consenso informato non acquisito o } \\
\text { acquisito in maniera non corretta, } \\
\text { tempestiva od esaustiva }\end{array}$ & $\begin{array}{l}\text { - Percorsi strutturati di formazione } \\
\text { - Audit di verifica e miglioramento }\end{array}$ \\
\hline
\end{tabular}




\section{I percorsi intraospedalieri: ammissione, organizzazione per intensità di cure e dimissione}

I percorsi di ammissione e dimissione coinvolgono non soltanto i reparti di medicina interna, ma l'intero ospedale, a partire evidentemente dal pronto soccorso. Ormai tutti i processi di accreditamento, di ospedale, di dipartimento, di unità operativa prevedono la presenza di percorsi ad hoc per l'allocazione di un determinato paziente in un determinato reparto e per il trasferimento dal pronto soccorso al reparto accettante.$^{8}$ Nonostante ciò la presenza di un per- corso di per sé non è sufficiente a garantire totalmente la sicurezza. È infatti opportuno che nei percorsi disegnati vengano ben definite le caratteristiche sia cliniche che di instabilità potenziale dei pazienti, in relazione alla dotazione tecnologica, agli organici e alla preparazione specifica dei diversi professionisti per il trattamento delle patologie ammissibili. Infatti ricoverare un paziente potenzialmente instabile in un reparto medicina interna non dotato di opportuni presidi per il monitoraggio delle funzioni vitali o di personale adeguatamente formato o numericamente insufficiente costituisce un elemento di importante rischio innanzitutto per il paziente stesso (rischio clinico propriamente detto), ma anche per la

Tabella 1. Segue dalla pagina precedente

\begin{tabular}{|c|c|c|}
\hline Ambito & Principali tipologie di errori (guasti) & Possibili correttivi \\
\hline Terapia & $\begin{array}{l}\text { - Difetto di ricognizione e riconciliazione } \\
\text { farmacologica } \\
\text { - Non adeguata leggibilità dei fogli di terapia } \\
\text { - Non adeguato e tempestivo aggiornamento } \\
\text { dei fogli di terapia } \\
\text { - Mancanza o fallimento di meccanismi } \\
\text { idonei alla corretta identificazione dei } \\
\text { pazienti ai quali associare le specifiche } \\
\text { terapie prescritte. } \\
\text { - Utilizzo di più supporti (cartacei, } \\
\text { informatici) per le stesse informazioni } \\
\text { - Problemi connessi al software (difetti, } \\
\text { interruzioni, macchinosità, carenze) } \\
\text { - Violazione dei passaggi obbligati del } \\
\text { software di gestione della terapia } \\
\text { - Non controllo diretto della assunzione } \\
\text { della terapia orale } \\
\text { - Problemi legati ai dispositivi medici } \\
\text { (cateteri, pompe, ecc.): difettosità dei dispo- } \\
\text { sitivi, uso non corretto o non appropriato }\end{array}$ & $\begin{array}{l}\text { - Adozione di sistemi per la prescrizione di } \\
\text { tipo informatizzato } \\
\text { - Percorsi e strumenti per la corretta } \\
\text { identificazione dei pazienti ed il corretto } \\
\text { abbinamento alla prescrizione terapeutica } \\
\text { (braccialetto con bar-code) } \\
\text { - Formazione del personale ed Audit di } \\
\text { verifica dedicati }\end{array}$ \\
\hline Igiene ambientale e misure di prevenzione & $\begin{array}{l}\text { - Assenza di idonee misure di isolamento } \\
\text { per soggetti colonizzati o infetti } \\
\text { potenzialmente contagiosi } \\
\text { - Adozione carente o scorretta di schemi di } \\
\text { profilassi antibiotica quando necessari } \\
\text { - Mancanza di sensibilizzazione e/o } \\
\text { adozione difettosa (assente o tardiva) di } \\
\text { protocolli igienico sanitari idonei al } \\
\text { contenimento del rischio infettivo (igiene } \\
\text { delle mani, camici, guanti, mascherine a } \\
\text { perdere qualora necessarie, comportamenti, } \\
\text { ecc.) }\end{array}$ & $\begin{array}{l}\text { - Adozione di percorsi e protocolli specifici } \\
\text { - Percorsi strutturati di formazione } \\
\text { - Audit di verifica e miglioramento }\end{array}$ \\
\hline Dotazione organica & $\begin{array}{l}\text { Rapporto operatori sanitari/pazienti-posti } \\
\text { letto inferiore alle reali necessità ed esigenze } \\
\text { in relazione anche al tipo di patologie e di } \\
\text { pazienti ricoverati }\end{array}$ & $\begin{array}{l}\text { Definizione di criteri di appropriatezza per } \\
\text { la allocazione delle risorse umane in } \\
\text { relazione alla tipologia, severità e } \\
\text { complessità dei pazienti ricoverati }\end{array}$ \\
\hline Assetti organizzativi & $\begin{array}{l}\text { - Assenza di definizioni organizzative } \\
\text { idonee finalizzate ad un adeguato utilizzo } \\
\text { delle risorse di personale e diagnostico } \\
\text { terapeutiche } \\
\text { - Modelli organizzativi non in grado di } \\
\text { rispondere efficacemente ai diversi gradi di } \\
\text { severità e complessità dei singoli pazienti } \\
\text { ammessi }\end{array}$ & $\begin{array}{l}\text { Adozione di piani di lavoro e piani di } \\
\text { attività idonei a realizzare la più appropriata } \\
\text { allocazione di risorse di personale per ogni } \\
\text { spazio temporale in relazione ad effettive e } \\
\text { reali esigenze }\end{array}$ \\
\hline
\end{tabular}


struttura che, in caso di contenzioso per un esito infausto troverebbe difficile difendere la sua posizione. Ulteriore fonte di possibili rischi sono i cosiddetti appoggi fuori reparto, con ricoveri in altre strutture di pazienti che altrimenti dovrebbero essere accolti in letti sovrannumerari. Alcuni ospedali, soprattutto di più ampie dimensioni, hanno cercato di affrontare il problema prevedendo aree dedicate nelle quali posizionare pazienti, al fine di individuare meglio il setting più idoneo di ammissione o nell'intento di potere attendere in sicurezza la disponibilità di un posto letto nel reparto più adatto. ${ }^{9}$

Molte unità operative prevedono una precisa definizione anche per i percorsi di dimissione dei pazienti. Solitamente questi prevedono la cascata delle azioni che devono essere messe in atto, ovvero la chiusura della cartella clinica, la redazione di una lettera di dimissione adeguata e chiara, la riconciliazione e la consegna dei farmaci e la loro spiegazione, il colloquio con il paziente dimesso e/o con i suoi familiari. ${ }^{10} \mathrm{Un}$ ambito trascurato è invece quello relativo alla sicurezza della dimissione. ${ }^{11,12}$ Nella Tabella 2 sono riportati quelli che potrebbero essere considerati i criteri essenziali di sicurezza per una dimissione. In alcuni ospedali di grandi dimensioni è stata realizzata un'area, da alcuni denominata $d i$ scharge room, ove posizionare in sicurezza pazienti già dimessi, in attesa della definitiva lettera di dimissione o dell' effettivo trasferimento a domicilio. ${ }^{9}$ In realtà tale organizzazione, più che presidio di sicurezza per la dimissione del paziente sembra maggiormente finalizzata a liberare più rapidamente $\mathrm{i}$ posti letto nei reparti, evitando ad altri ammalati tempi di permanenza eccessivi presso il pronto soccorso o le sue aree adiacenti. Anche questo aspetto potrebbe pertanto ulteriormente configurarsi come in grado di ridurre il rischio clinico.

Il modello organizzativo per intensità di cure, sempre più diffuso nelle nostre strutture ospedaliere, anche se non in tutti i casi in maniera efficace, ${ }^{13}$ viene fortemente caldeggiato non solo dai professionisti, ma anche dai decisori della programmazione sanitaria. ${ }^{14}$
Esso, se applicato in maniera corretta, potrebbe costituire elemento utile al contenimento del rischio clinico. ${ }^{15}$ Questo modello è disegnato per potere allocare ciascun paziente nel setting più appropriato, sia per la dotazione organica, in termini di quantità e preparazione del personale che di appropriatezza tecnologica. Questi modelli solitamente prevedono la suddivisione dell'organizzazione in 3 livelli di intensità. ${ }^{15,16} \mathrm{Il}$ più alto livello (cosiddetto di alta intensità di cura) presuppone una dotazione tecnologica di monitoraggio multiparametrico, supporti ventilatori non invasivi ed altri presidi tecnologici (ecografia bedside, ecc.), in grado di supportare la gestione di pazienti con un discreto grado d'instabilità e di severità clinica. Le problematiche principali riferite a questo modello e sulle quali vi è anche tuttora un ampio dibattito in letteratura riguardano prevalentemente la sua strutturazione. Questa, in relazione al coinvolgimento di più unità operative, necessita di un forte potere di coordinamento e collegamento tra le stesse e potrebbe trovare la più piena attuazione nel modello dipartimentale. ${ }^{17,18}$ Un ulteriore elemento, decisivo per una corretta applicazione dell' organizzazione per intensità di cure, è la definizione di criteri condivisi per il giudizio dell'allocazione dei pazienti nelle diverse aree stratificate per severità. ${ }^{19}$ La maggior parte degli autori concorda sulla necessità di utilizzare criteri clinici che possono essere supportati, in talune situazioni, da score opportunamente disegnati. ${ }^{20}$ Tra quelli proposti, ancora oggi il modified early warning score (MEWS) sembra essere quello più diffuso e parzialmente validato, anche se il suo utilizzo prevalente e più riconosciuto rimane finalizzato al monitoraggio infermieristico della situazione clinica di pazienti già ricoverati. Oltre alla necessità di definire i criteri di una giusta allocazione dei pazienti nei diversi setting assistenziali si avverte anche l'esigenza di definire criteri espliciti sulle modalità di trasferimento dei pazienti tra le diverse aree di intensità, in quanto in questi modelli un paziente che abbia superata la fase più acuta e di maggiore instabilità dovrebbe essere spostato in una delle aree a minor intensità.

Tabella 2. Condizioni essenziali per una dimissione in sicurezza da un reparto ospedaliero.

\author{
Assolute \\ Stabilità clinica ottenuta da almeno $24-48$ ore \\ Non necessità od opportunità di trattamenti complessi o di difficile esecuzione domiciliare \\ Non necessità di indagini diagnostiche per le quali sia indispensabile la ospedalizzazione \\ Ripristino di condizioni di autonomia accettabili, o comunque le più vicine possibili allo stato del pre-ricovero \\ Relative \\ Non prevedibili ulteriori benefici dal prolungamento della degenza \\ Disponibilità del paziente e/o dei familiari alla dimissione \\ Ripristino di condizioni di autonomia parziali e non ulteriormente migliorabili con un prolungamento della degenza \\ Capacità di assunzione di terapia in autonomia o presenza di caregiver adeguato allo scopo \\ Documentata presenza degli opportuni presidi sanitari se indispensabili per la assistenza \\ Non ulteriore necessità di controlli clinici e/o bioumorali/strumentali a breve termine
}




\section{Percorsi di dimissione e dimissione protetta}

Oltre alla definizione di una opportuna check list (Tabella 3) di azioni da seguire nell'effettuare la dimissione di un paziente in sicurezza, altre modalità possono essere previste ai fini di ridurre il rischio clinico nel percorso di dimissione. La dimissione pro$t e t t a^{21}$ si riferisce generalmente ad un percorso in cui la preparazione della dimissione viene condivisa tra struttura ospedaliera e territorio, con il coinvolgimento del medico di famiglia, del servizio infermieristico domiciliare o della geriatria territoriale. L'obiettivo è quello di creare le condizioni ideali o per il reinserimento di un paziente fragile in famiglia o per un suo temporaneo ricovero in una struttura intermedia, ad esempio in una residenza sanitaria assistenziale. Alternative a tali soluzioni potrebbero essere rappresentate dai cosiddetti ospedali di comunità o dalle case della salute, in via di attivazione in molte Unità Sanitarie Locali. ${ }^{22}$ In realtà, il termine dimissione protetta potrebbe includere qualsiasi modalità di presa in carico in grado di realizzare una gestione intermedia tra l'ospedale e il territorio; ad esempio, un percorso ambulatoriale complesso, quale il day service, ${ }^{23}$ in cui prevedere un approfondimento delle indagini diagnostiche e, se necessario, od un opportuno follow-up clinico o terapeutico.

\section{I percorsi clinico assistenziali per le patologie più frequentemente causa di ricovero}

Nell'epoca della medicina basata sull'evidenza e della disponibilità di una tecnologia sempre più complessa non è più possibile pensare che pazienti affetti dalle stesse patologie vengano trattati in maniera estremamente disparata a seconda del professionista che ha in cura quel determinato paziente. ${ }^{24,25}$ Per ovviare a questa problematica, con l'obiettivo di utilizzare le indicazioni che provengono dalla medicina basata sulla evidenza, le linee guida migliori disponibili in letteratura e l'esperienza degli operatori, stanno cominciando a diventare strumento quotidiano della pratica medica ospedaliera i cosiddetti percorsi diagnostico assistenziali o diagnostico terapeutici (PDTA) ${ }^{26} \mathrm{Si}$ tratta di percorsi costruiti in condivisione da gruppi di lavoro e discussi poi con le équipes medico- infermieristicheassistenziali che dovranno utilizzarli. Essi, oltre che definire linee di comportamento e le loro basi scientifiche, si estrinsecano nella costruzione di una checklist da seguire e documentare nel suo percorso di utilizzo durante il periodo di ricovero. ${ }^{27}$ Tale processo diventa quindi anche una modalità in grado di documentare l'aderenza al percorso clinico-assistenziale costruito per quella determinata patologia. Come tutti i processi documentativi potrà poi essere oggetto di epicrisi e rivalutazione in opportuni audit clinici. ${ }^{28}$ Questi, come è ben noto, costituiscono il più opportuno strumento di verifica dell'applicazione di questi percorsi e diventano lo strumento più idoneo per verificare l'adesione degli operatori alle pratiche previste dal PDTA e per potere applicare eventuali correttivi allo stesso. ${ }^{29} \mathrm{La}$ complessità del paziente internistico è tuttavia tale che l'applicazione dei protocolli e delle checklist previste dai PDTA deve essere sempre tagliata sul singolo paziente. L'abilità dell'internista deve quindi misurarsi sulla sua capacità di coniugare esperienza, linee guida, percorsi e protocolli e disegnare quindi per ogni paziente l'iter diagnostico e la terapia più appropriata alle sue caratteristiche ed al suo stato..$^{27,30}$

Tabella 3. Checklist delle azioni per la corretta dimissione di un paziente.

La dimissione di un paziente non è una cosa banale: molti pazienti incorrono in eventi avversi dopo la dimissione e una quota di pazienti intorno al 20\% viene nuovamente ricoverata entro 30 giorni dal rientro a domicilio

i) Preavviso almeno 24 prima al paziente e/o ai suoi familiari o caregiver

ii) Consegna della lettera da parte del personale sanitario al paziente o ai suoi familiari o caregiver

iii) Consegna dei farmaci prescritti

iv) Colloquio esplicativo relativo a:

- Motivi della ospedalizzazione,diagnosi effettuata e provvedimenti messi in atto

- Comportamenti o modifiche dello stile di vita

- Segni o sintomi motivo di allerta o di necessità di nuovo contatto con il medico curante o con la struttura sanitaria

- Modalità ed estremi per un nuovo contatto, qualora necessario

- Terapie prescritte, motivazioni, modalità di assunzione ed avvertenze e precauzioni

- Controlli programmati e loro modalità

v) Eventuale contatto con il medico di famiglia, se opportuno

vi) Verifica della sussistenza di tutte le condizioni previste dalla dimissione protetta, qualora attivata

- Informazione al medico curante

- Comunicazioni e materiale di raccordo al servizio infermieristico domiciliare ed ai servizi sociali

Modificata da Ann Intern Med 2013;158:397-403 


\section{La corretta compilazione}

\section{della documentazione sanitaria}

La documentazione sanitaria e la sua corretta tenuta stanno acquisendo sempre più importanza, ${ }^{31}$ sia per le attività cliniche, che per ragioni epidemiologicostatistiche, medico-legali, amministrative e ai fini della valutazione della qualità. ${ }^{32}$ La diligenza nella registrazione dei dati non deve essere fine a sé stessa. Essa dovrebbe essere finalizzata a riflettere, in modo sintetico ma esaustivo, la qualità dell'approccio assistenziale ad ogni specifico problema. Il metodo CLAP presuppone un approccio alla compilazione delle cartelle e cliniche e delle schede infermieristiche finalizzate alla Completezza, Leggibilità, Accuratezza e Professionalità nella registrazione dei dati, ai fini di buone registrazioni sia dei medici che degli infermieri (Tabella 4). I dati della cartella, se chiari ed essenziali, sono proficui anche per le successive valutazioni di audit clinico e medico-legali. La povertà (o la ricchezza) delle registrazioni riportate inducono, inevitabilmente, giudizi, negativi o positivi, sulla validità dell'assistenza prestata. Le correzioni dovrebbero essere evitate. L'impiego dei bianchetti o dei pennarelli va proscritto. Se necessario è meglio tracciare una semplice linea trasversale sulla parte da annullare, in modo tale da non dare l'impressione di voler occultare o eliminare i dati trascritti. In sostanza, un'adeguata e corretta tenuta della documentazione sanitaria non può essere soltanto finalizzata alla difesa di ipotetici rischi di contenzioso civile o penale, ma dovrebbe essere correlata alla qualità del lavoro, ai percorsi diagnostico assistenziali intrapresi, all'appropriatezza e alla continuità assistenziale e terapeutica proposta, nel contesto di un lavoro di équipe multi-professionale.

\section{La cartella clinica integrata}

La documentazione sanitaria per un paziente ricoverato è costituita dalla cartella clinica. Tale documento, fino ad alcuni anni fa considerato esclusivamente di pertinenza medica, oggi con le nuove attribuzioni infermieristiche sta sempre più diventando uno strumento d'integrazione medico-infermieristica. ${ }^{33}$ Diverse esperienze di cartelle integrate sono state realizzate negli ultimi anni, talune anche in formato elettronico. In ambito internistico, in maniera ancora più peculiare, la struttura della cartella clinica ha avuto una significativa evoluzione, fino alla possibilità di declinare le complesse problematiche dei pazienti e disegnare un possibile percorso, adeguato alla centralità dei loro bisogni. Oltre ai tradizionali rilevi relativi all'anamnesi (familiare, fisiologica, patologica remota, patologica recente) sono stati introdotti altri campi importanti ai fini di una dimissione protetta per i pazienti più fragili ed anziani. Il campo anamnesi sociale, unitamente a quello dei presidi in uso, diventa così di essenziale nel contesto di una documentazione sanitaria completa. A fianco di questi nuovi riferimenti anamnestici vi è la possibilità di inserire altri dati obbligatori, quali, ad esempio, le allergie e le intolleranze, in taluni casi associate a specifici alert generati in maniera automatica e facilmente osservabili dai professionisti coinvolti nella gestione del paziente. Un'ulteriore opportunità è data dalla possibilità di compilazione automatica o semi automatica dei punteggi di valutazione più opportuni in relazione alla patologia $\mathrm{o}$ alla situazione clinica di un determinato paziente ( $\mathrm{sco}$ res di severità - ad esempio, MEWS o similari, classi di gravità - ad esempio NYHA, score di rischio tromboembolico, score di patologia, ecc.). ${ }^{33}$ La realizzazione di esperienze elettroniche, con l'utilizzo di sistemi quali, ad esempio, la firma digitale, può contribuire ulteriormente alla validazione in termini medico legali della documentazione sanitaria ed alla sua chiarezza, in grado di superare il rischio di annotazioni non congruenti in termini temporali e di una minor leggibilità dovuta a problemi calligrafici. ${ }^{34} \mathrm{La}$ fruibilità di una cartella elettronica diventa massima in quanto tutti gli operatori possono accedervi dal proprio terminale: ciò facilita la comunicazione e lo scambio di informazioni tra tutta l'équipe medica ed infermieristica. La cartella clinica elettronica può costituire un supporto formidabile per leggibilità, fruibilità delle informazioni sanitarie, anche ai fini della continuità e del coordinamento delle cure dopo la dimissione. Tuttavia l'adesione degli operatori e il loro apporto motivato a una esaustiva e completa compilazione rimane elemento essenziale per la sua ef-

Tabella 4. Utilità di buone registrazioni nella documentazione sanitaria.

Per provvedere una esauriente consegna agli altri membri della equipe ai fini della prosecuzione della assistenza del paziente e suo del follow-up i) Favorisce il lavoro di equipe

Per potere disporre di dati oggettivi, ai fini di richiami anamnestici e per poter riferire, in modo corretto su fatti avvenuti in precedenza o su dettagli che possono essere dimenticati (anche ai fini testimoniali)

i) Permette una rivalutazione critica dei casi

ii) È un utile strumento di difesa medico legale

Per una maggiore tutela legale di fronte ad eventuali conflitti o reclami, onde potere evidenziare le azioni, le decisioni, l'assistenza e le opinioni assunte nei singoli casi in modo completo 
ficacia operativa. Sono pertanto necessari adeguati momenti di condivisione, sia nella fase di preparazione che di implementazione in ogni singola équipe, al fine di sfruttarne pienamente le potenzialità e promuoverne l'utilizzo da parte di tutti gli operatori coinvolti.

\section{La prescrizione e la somministrazione della terapia}

L'atto terapeutico costituisce uno degli elementi essenziali nel processo di assistenza. Esso si compone fondamentalmente di tre parti: la prima, di competenza medica, è la prescrizione; la seconda è la preparazione del farmaco, a seconda delle circostanze di competenza dell'infermiere o del farmacista, e infine la somministrazione, di competenza infermieristica. A queste è stata aggiunta da alcuni anni la cosiddetta riconciliazione farmacologica, che comprende a sua volta l'atto della ricognizione (di competenza di un professionista sanitario: medico, infermiere o farmacista) e la riconciliazione vera e propria. La Riconciliazione è il processo nell'ambito del quale si confrontano i farmaci assunti dal paziente, così come evidenziati nella Ricognizione, con quelli indicati per la cura nella particolare circostanza, in funzione di una decisione prescrittiva corretta e sicura. Il percorso di Riconciliazione prevede che il medico prescrittore, nell'eseguire il confronto, valuti attentamente l'opportunità del prosieguo del trattamento farmacologico in atto e la sua compatibilità con quello che ritiene indicato nella specifica circostanza. ${ }^{35}$ Gli errori nella fase della riconciliazione sono ritenuti particolarmente frequenti e di potenziale grave impatto sul percorso di cura.

A prescindere dalle problematiche correlate alla fase della riconciliazione farmacologica, all'appropriatezza prescrittiva e agli errori connessi, altre importanti fonte di errore possono essere correlate a confondimenti per confezione simile o nome assonante (cosiddetti LASA: Look Alike, Sound Alike, cioè appare simile, suona simile), ad imprecisioni, non chiarezza o scarsa leggibilità del foglio di terapia cartaceo ${ }^{36,37} \mathrm{La}$ fase relativa alla preparazione e alla somministrazione si presta altresì ad ulteriori possibili rischi, quali uno stoccaggio non differenziato per confezioni simili, una non corretta diluizione del farmaco in fase di preparazione, la somministrazione errata di un farmaco al paziente sbagliato, la non verifica della corretta assunzione della terapia da parte di pazienti anziani poco collaborativi. ${ }^{38}$ Nuovi sistemi di tipo elettronico, recentemente implementati in diverse aziende sanitarie, si sono rivelati in grado, anche se non completamente, di ridurre alcuni dei rischi sopraelencati. In particolare, nella fase di prescrizione sono pressoché aboliti i rischi connessi alla non leggibilità del farmaco prescritto. ${ }^{17,33}$ L'impiego di un braccialetto elettronico identificativo può ridurre il rischio di som- ministrazione di un farmaco al paziente sbagliato. Tali strumenti, purtroppo, non eliminano i rischi determinati dalla mancata sorveglianza da parte degli operatori, specie infermieri, della corretta assunzione della terapia prescritta. ${ }^{39}$

\section{Politerapia: rischi e interventi per il suo contenimento}

Non è molto frequente vedere trattare questo tema nell'ambito del rischio clinico, forse perché raramente dà luogo a contenzioso economico o giudiziario. Tuttavia il numero di persone, soprattutto anziane, in politerapia (intesa generalmente come un numero giornaliero di farmaci superiore a cinque) è enorme. In uno studio condotto nel nord Italia nel 2008, oltre la metà dei pazienti con più di 65 anni ricoverati in un'Unità di Medicina interna o Geriatria per una qualunque causa assumeva regolarmente almeno cinque diverse specialità medicinali con obbligo di prescrizione. ${ }^{40}$ Molti studi hanno documentato che la politerapia è associata con una serie di outcome negativi, soprattutto nell'anziano: ${ }^{41}$ aumento della probabilità di interazioni farmacologiche, diminuzione della compliance, aumento di probabilità di errore nella somministrazione (in reparto o struttura socio-assistenziale) o nell'assunzione (a domicilio), aumento della probabilità di cadute. Inoltre, non raramente la politerapia nasconde inappropriatezza prescrittiva, specie per alcune classi di farmaci, come antiaritmici, PPI, psicofarmaci. L'adozione di una politica di contenimento del numero di farmaci è quindi uno degli strumenti che dovrebbero concorrere a contenere il rischio clinico. Operativamente, può estrinsecarsi in diversi punti, ${ }^{42,43}$ quali ad esempio: considerare nella fase diagnostica l'ipotesi di quadri clinici attribuibili a danno da farmaci; identificare e sospendere eventuali farmaci inappropriati o comunque non necessari; limitarsi alla prescrizione di farmaci secondo l'interpretazione più ampia del termine appropriatezza (che comprende efficacia, sicurezza, accettabilità, ecc.), specialmente quando si supera il numero di cinque specialità medicinali giornaliere.

\section{Le misure igienico sanitarie nella prevenzione del rischio infettivo e delle infezioni nosocomiali}

Il problema delle infezioni ospedaliere e della possibilità di contrarre infezioni collegate alle cure sanitarie è esploso negli ultimi anni, non soltanto in ambiente chirurgico, ma anche in quello internistico. ${ }^{44}$ Si tratta di infezioni che, specie in ambito medico, sono nella maggioranza dei casi prevenibili, con la messa in atto di opportune semplici misure precauzionali, quali il lavaggio delle mani e l'utilizzo di stru- 
menti idonei a ridurre i contatti con materiale infetto. In alcune realtà queste misure sono oggetto di opportuni audit con l'obiettivo specifico del contenimento di questo rischio. ${ }^{45,46}$ Parallelamente, anche se con maggior fatica, si sta sviluppando la consapevolezza che $\mathrm{i}$ rischi correlati alle cure sanitarie possono in parte dipendere anche da un utilizzo non sempre congruo ed adeguato degli strumenti terapeutici a nostra disposizione. Un capitolo di particolare importanza è quello relativo alla cosidetta stewardship della antibioticoterapia. ${ }^{47-49}$ È sempre più frequente infatti riscontrare, soprattutto in pazienti fragili o immunodepressi, la presenza - o come colonizzanti o come causa di infezioni spesso severe, germi cosiddetti multiresistenti, quali MRSA (methicillin-resistant Staphylococcus aureus), gram negativi ESBL (extended-spectrum betalactamase) o KPC (Klebsiella pneumoniae carbapenemase) produttori. Insieme alle diarree da Clostridium difficile la diffusione di questi germi è strettamente correlata alla pressione antibiotica sul nostro ecosistema batterico. Tutte le politiche finalizzate al corretto uso della terapia antibiotica e alla corretta gestione dei pazienti affetti da infezioni o colonizzazioni nosocomiali sono da considerare indispensabili per una corretta gestione del rischio infettivo anche nei reparti di medicina interna, ove sempre più spesso giungono pazienti portatori di infezioni correlate all'assistenza che, se non gestiti correttamente, possono essere forieri di ulteriori trasmissioni ad altri pazienti fragili ricoverati nelle loro adiacenze. ${ }^{50}$

\section{Dotazione organica, assetti organizzativi, sovraffollamento dei reparti, distribuzione e preparazione del personale}

Uno dei problemi meno considerati, ma frequentemente alla radice di eventi indesiderati, riguarda la dotazione organica ed il grado di qualificazione e preparazione dei professionisti e degli operatori presenti. Tali aspetti attengono non soltanto alla numerosità del personale in servizio, ma anche alla sua distribuzione nei diversi periodi temporali giornalieri, settimanali e mensili. Aiken e coll. ${ }^{51}$ nel febbraio 2014 hanno pubblicato un interessante lavoro nel quale si concludeva come un sovraccarico di lavoro sul personale infermieristico di assistenza si traducesse in un significativo incremento della probabilità di morte a 30 giorni. Nello stesso lavoro e nell'editoriale collegato ${ }^{52}$ si sottolineava anche come un ruolo importante nel condizionare gli esiti migliori o peggiori per i pazienti ricoverati fosse giocato anche dal grado di preparazione e qualificazione del personale addetto. Un altro aspetto di rilievo riguarda l'organizzazione dei reparti nel weekend, che comporta spesso diverse modalità di gestione rispetto alle giornate infrasettimanali e outcome diversi. ${ }^{53,54}$ Studi di ampie dimensioni, anche se per la maggior parte retrospettivi, hanno dimostrato come l'essere ricoverati in ospedale nel fine settimana comporti una probabilità di morte e di esito negativo fino ad una volta mezzo rispetto alle altre giornate. ${ }^{53}$ Anche se non direttamente dimostrato, si potrebbero ipotizzare come fattori in grado di concorrere a questo esito peggiore, la minor presenza di personale in servizio in questo periodo temporale del weekend, un minor grado di continuità assistenziale dovuta all'avvicendarsi dei diversi medici di guardia e a la minore disponibilità di tecnologie diagnostiche. Altri Autori hanno analizzato il rischio correlato al sovraffollamento, molto frequente in alcuni reparti di medicina interna, ${ }^{55,56}$ identificandolo come importante fattore di rischio di eventi avversi, ${ }^{57-59}$ particolarmente nell'ambito dei dipartimenti di emergenza urgenza. Le cause sottese a tale sono costituite essenzialmente da incremento di errori nella terapia, incompletezza e non esaustività della documentazione sanitaria, incremento del rischio infettivo, ritardi diagnostici e conseguentemente anche di impostazione terapeutica. Molti dati in effetti confermano che gli ospedali sovraffollati sono meno sicuri, con possibilità di mortalità precoce e di breve termine più elevata. ${ }^{60,61} \mathrm{Ad}$ esempio le infezioni da MRSA sono strettamente correlate, in particolare nei reparti di Medicina Generale, ai picchi di aumento, in alcuni casi fino al $120 \%$, dell'indice di occupazione, verosimilmente in rapporto a una minore attenzione da parte del personale alle norme igieniche. ${ }^{62}$ Il problema del sovraffollamento dei reparti internistici è particolarmente critic $^{63} \mathrm{e}$ tale da richiedere interventi di tipo strutturale ed organizzativo non soltanto su un singolo reparto, ma sull'ospedale nella sua complessità e nelle sue articolazioni dipartimentali, nell'intento di perseguire una più globale politica di gestione dei posti letto. Probabilmente un modello organizzativo strutturato per intensità di cure ed eventualmente anche una macro-aggregazione dipartimentale, ${ }^{15,17}$ potrebbe dare risposte a queste problematiche. Altri interventi proposti e, almeno in parte, in alcune realtà già implementati, sono quelli finalizzati a migliorare l'appropriatezza dei ricoveri, strutturando percorsi alternativi quali aree di osservazione breve, ${ }^{64}$ di degenza breve, ${ }^{65}$ percorsi diagnostico assistenziali in regimi di tipo ambulatoriale complesso quali il day service ${ }^{23}$ e percorsi integrati ospedale territorio per le più frequenti patologie croniche. ${ }^{66}$

Sul versante opposto, quello delle dimissioni, come già accennato in precedenza, un'adeguata e precisa strutturazione delle cosiddette dimissioni protette ${ }^{21}$ potrebbe essere strategica al fine di evitare o quantomeno ridurre il più possibile il fenomeno dei cosiddetti Bed blockers,${ }^{67}$ cioè di quei pazienti potenzialmente dimissibili che per ragioni prevalentemente di tipo socio assistenziale e non clinico non riescono ad essere realmente dimessi nei tempi previsti ed opportuni. 
Ultimo, ma non in termini di priorità, è da considerare, in questo ambito, l'aspetto formativo. Come dimostrato e sottolineato da alcuni lavori la preparazione e la qualificazione del personale addetto è un altro elemento che deve essere considerato come inversamente proporzionale al rischio clinico. ${ }^{51}$ Pertanto ogni strutturazione ed assetto organizzativo deve evidentemente prevedere un'adeguata formazione e preparazione del personale in relazione alla tipologia dei pazienti afferenti, alle tecnologie usate ed in dotazione ed ai percorsi clinico assistenziali presenti. Si tratta di una formazione che deve essere considerata come di tipo permanente in quanto è assolutamente consolidato il fatto che debba essere oggetto periodicamente di opportuni retraining. Questi potranno essere opportunamente guidati focalizzati alla luce anche dei dati ricavabili dagli audit clinici di verifica dei percorsi e delle prestazioni erogate.

\section{Trasferimenti extra-interospedalieri: una problematica per le Medicine degli ospedali di piccole medie dimensioni}

Il trasferimento di un paziente acuto da un ospedale ad un altro solitamente avviene a motivo di condizioni cliniche per le quali sono necessarie competenze specialistiche o tecnologie di monitoraggio e/o diagnostiche non presenti all'interno della struttura inviante. ${ }^{68} \mathrm{Si}$ tratta di pazienti che provengono da ospedali di piccole medie dimensioni (centri spoke), ove la Medicina Interna funge da reparto portante. Il trasferimento avviene verso un ospedale di più grandi dimensioni o specializzato, nel contesto di quelle che oggi vengono definite reti ospedaliere. Le problematiche ed i rischi connessi al trasferimento di un paziente acuto od instabile riguardano prevalentemente la definizione delle modalità più sicure per il trasporto: ${ }^{69,70}$ quale mezzo e con quali dotazioni tecnologiche, quale figura professionale (internista, anestesista rianimatore, infermiere specializzato, ecc.) (Tabella 5). In letteratura le esperienze riportate non

Tabella 5. Checklist per i trasferimenti interospedalieri.

Il paziente è stabile?

La sede di destinazione adeguata al caso?

Esistono bisogni specifici per il paziente?

Chi e come accompagnerà il paziente durante il trasporto?

Sono stati valutati i benefici/rischi?

Sono rispettate le preferenze del paziente?

Il posto letto è disponibile?

Comunicazione con l'equipe ricevente sono molte e non contemplano specificamente i reparti di Medicina Interna. In ambito anestesiologico si è cercato di definire con la maggiore accuratezza possibile attraverso opportuni protocolli le modalità più idonee in relazione alle figure professionali presenti o attivabili. ${ }^{71}$ Questo rimane comunque un capitolo che, in una logica di contenimento del rischio clinico, deve essere opportunamente considerato ed affrontato da ogni singolo ospedale.

\section{Conclusioni}

La dimensione del rischio clinico in ambito sanitario è acquisizione relativamente recente, oggetto di una continua e costante implementazione negli ultimi anni. Parallelamente vi è una presa di coscienza del problema da parte non soltanto dei gestori ma anche da parte dei professionisti addetti all'assistenza, in particolare nell'ambito della Medicina Interna. Da qui lo sviluppo di analisi e di settori sempre nuovi, a fianco dei quali sta iniziando un confronto, per elaborare risposte anche pratiche, attinenti all'organizzazione macro e micro, alla pratica quotidiana, alla realizzazione di strumenti operativi, quali, ad esempio, la cartella clinica elettronica.

\section{Bibliografia}

1. Pazzi M. Rischio clinico nel diritto dell'Unione europea. Politiche Sanitarie 2013;14:85-90.

2. Cinotti R, ed. Processo e strumenti della gestione della sicurezza. In: La gestione del rischio nelle organizzazioni sanitarie. Roma: Il Pensiero Scientifico Editore; 2004. p 35.

3. Soccetti A. Il corretto dialogo fra mondo sanitario e mercato assicurativo: il contributo del risk manager. Presentazione al convegno "Risk management in Ospedale: tra governance e assicurazioni”, Milano, 21 Giugno 2012.

4. Nardi R, Berti F, Greco A, et al. Complexity in hospital internal medicine departments: what are we talking about? Ital J Med 2013;7:142-55.

5. Salvioli G, Foroni M. La Medicina della complessità, la Medicina Interna e la Geriatria Editoriale. G Gerontol 2008;56:1-10.

6. Kohn LT, et al. To err is human: building a safer health system. Institute of Medicine (IOM), Quality of Health Care in America project Report 1. December 1999. National Academy Press; 1999.

7. Charles V. La sicurezza del paziente. Berlin: Springer; 2011. pp 212-13.

8. Agenzia Sanitaria Regionale Emilia Romagna. Requisiti specifici per l'accreditamento delle strutture di Medicina Interna. Delibera ${ }^{\circ} 419$ del 20/04/2012, pp 28-29.

9. Mazzi G. L'esperienza dell'area medica. Ospedale Santa Maria Nuova Reggio Emilia in Tavola Rotonda "Ospedale per Intensità di cura : opportunità e criticità" Convegno Nazionale Modelli di Assistenza Ospedaliera per 
Intensità di Cura: esperienze nazionali ed internazionali, Bologna, 29-30 Marzo 2012.

10. Agenzia Sanitaria Regionale Emilia Romagna. Requisiti specifici per l'accreditamento delle strutture di Medicina Interna. Delibera n ${ }^{\circ} 419$ del 20/04/2012, pp 31-32.

11. McDonagh MS, Smith DH, Goddard M. Measuring appropriate use of acute beds. Asystematic review of methods and results. Health Policy 2000;53:157-84.

12. Fontaine P, Jacques J, Gillain D, et al. Assessing the causes inducing lengthening of hospital stays by means of the appropriateness evaluation protocol. Health Policy 2011;99:66-71.

13. Nardi R, Arienti V, Nozzoli C, Mazzone A. Organizzazione degli ospedali per intensità di cure: gli errori da evitare Ital J Med 2012;6:1-13.

14. Stefani I, Mazzone A. Come organizzare l'Area medica per affrontare le nuove sfide della complessità clinica ed assistenziale. Quad It J Med 2014;2:61-6.

15. Chesi G, Boni F. Ospedali e modelli organizzativi per intensità di cure: il punto di vista dell'Internista. Ital J Med 2012;6:63-71.

16. Agenzia Sanitaria Regionale Emilia Romagna. Requisiti specifici per l'accreditamento delle strutture di Medicina Interna. Delibera ${ }^{\circ} 419$ del 20/04/2012, pp 12-14.

17. Chesi G, Maccari S, Manicardi V, et al. Considerazioni in merito alla costruzione di un Dipartimento di Area Internistica nella AUSL di Reggio Emilia: proposta per una metodologia di lavoro. Politiche Sanitarie 2014; 15:36-46.

18. Cicchetti A, Ghirardini A, Mascia D, et al. Il modello organizzativo dipartimentale nel Servizio sanitario nazionale: risultati dell'indagine nazionale. Politiche Sanitarie 2009;10:153-68.

19. Scotti E, Pietrantonio F. The hospital Internal Medicine specialist today: a literature review and strength, weaknesses, opportunity, threats (SWOT) analysis to develop a working proposal. Ital J Med 2013;7:278-86.

20. Chesi G, Nardi R. Severity stratification of patients hospitalized on the Internal Medicine ward: work in progress. Ital J Med 2013;7:231-3.

21. Agenzia Sanitaria Regionale Emilia Romagna. Requisiti specifici per l'accreditamento delle strutture di Medicina Interna. Delibera n 419 del 20/04/2012, pp 32-34.

22. Brambilla A. Ospedale di comunità: un "ponte" per l'assistenza sanitaria intermedia Newsletter Saluter Notizie. 2013;10:16. Disponibile a: http://www.saluter.it/news/ newsletter/2013/ospedale-di-comunita-un-nuovo-modello-per-1 assistenza-sanitaria-intermedia

23. Zini D. Un modello di assistenza specialistica ambulatoriale complessa: il Day Service Nuovi modelli di continuità diagnostico-terapeutica relazione. I Conferenza Nazionale sulle Cure Primarie, Bologna, 25-26 Febbraio 2008; disponibile a: http://www.saluter.it/documentazione/convegni-e-seminari/conferenza-nazionale-cureprimarie/zini

24. Pagliaro L, Cappello M, Malizia G, et al. La medicina delle prove di efficacia nell'assistenza al paziente individuale. In: Liberati A, ed. La medicina delle prove di efficacia: utilità e limiti della evidence-based medicine. Roma: Il Pensiero Scientifico Editore; 1997. pp 33-56.

25. Pagliaro L. La medicina basata sull'evidenza. Rec Prog Med 1998;89:109-13.

26. Adeyemi S, Demir E, Chaussalet T. Towards an evi- dence-based decision making healthcare system management: modelling patient pathways to improve clinical outcomes. Dec Support Syst 2013;55:117-25.

27. Nardi R, Fabbri T, Belmonte G, et al. Internal medicine, complexity, evidence based medicine, almost "without evidences". Ital J Med. 2009;3:191-200.

28. van de Klundert J, Gorissen P, Zeemering S. Measuring clinical pathway adherence. J Biomed Inform 2010;43: 861-72.

29. van Herk R, Klazinga NS, Schepers RM, Casparie AF. Medical audit: threat or opportunity for the medical profession. A comparative study of medical audit among medical specialists in general hospitals in The Netherlands and England, 1970-1999. Soc Sci Med 2001;53: 1721-32.

30. Nardi R, Scanelli G. Evidence based medicine is broken? As internist we don't think so, but... there is a real world. BMJ 2014;348:g22.

31. Nonis M, Braga M, Guzzanti E. Cartella clinica e qualità dell'assistenza. Roma: Il Pensiero Scientifico Editore; 1998.

32. Damen V. Qualità della documentazione sanitaria, incident reporting e rischio clinico. Presentazione orale, Baveno, 29-30 giugno 2007.

33. Chesi G, Maccari S, Ligabue R, e al. Cartella clinica informatizzata per un Dipartimento di area internistica organizzato per intensità di cure: strumento per migliorare l'appropriatezza e la qualità delle cure sanitarie. Politiche Sanitarie 2012;13,2:109-24.

34. Hayrinen K, Saranto K, Nykanen P. Definition, structure, content, use and impacts of electronic health records: a review of the research literature. Int J Med Inf 2008;77:291-304.

35. Regione Emilia-Romagna (A cura del Gruppo Regionale sul Rischio clinico da farmaci). Raccomandazione Regionale per la Sicurezza nella terapia farmacologica n.2, Maggio 2011.

36. Nardi R, Borioni D, Pasquale A, et al. Nella Medicina Interna ospedaliera vogliamo un sistema sanitario pubblico sostenibile: considerazioni e proposte di FADOI per ridurre le spese inappropriate. Quad It J Med 2014;2:29-4.

37. Ciampalini S, Rinaldi M, Banfi R, et al. Rilevazione delle metodologie del percorso del farmaco in ospedale nelle aziende sanitarie della regione Toscana: identificazione dei principali fattori di rischio di errori in terapia. Atti del XXVI congresso Sifo Catania 19-22 ottobre 2005. Giorn Ital Farm Clin 2005;19:3.

38. Regione Toscana - Centro Gestione Rischio Clinico (a cura di Bellandi T., Ranzani A.). I quaderni per le campagne della sicurezza del paziente Scheda Terapeutica Unica. Disponibile a: http://www.regione.toscana.it/documents/10180/603668/Scheda+terapeutica+unica.pdf/a 3044306-2a7a-46d9-9673-6fbbda238d9e

39. Reed-Kane D, Vasquez K, Pavlik A, et al. E-prescription errors and their resolution in a community compounding pharmacy. Int J Pharm Compd 2014;18: 159-61.

40. Nobili A, Licata G, Salerno F, et al. Polypharmacy, length of hospital stay, and in-hospital mortality among elderly patients in internal medicine wards. The REPOSI study. Eur J Clin Pharmacol 2011;67:507-19.

41. Hajjar ER, Cafiero AC, Hanlon JT. Polypharmacy in elderly patients. Am J Geriatr Pharmacother 2007;5:345-51.

42. Nobili A, Pasina L, Mangiagalli A, et al. Farmaci e an- 
ziani - Metodi per gestire l'inappropriatezza prescrittiva. Dialogo sui Farmaci 2012;3:112-20.

43. Cartabellotta A. Sospendere i farmaci negli anziani: un approccio evidence-based. Evidence 2013;5:e1000061.

44. Magill SS, Edwards JR, Fridkin SK; Emerging Infections Program Healthcare-Associated Infections and Antimicrobial Use Prevalence Survey Team. Survey of health care-associated infections. N Engl J Med 2014;370:2542-3.

45. European Centre for Disease Prevention and Control. Point prevalence survey of healthcare associated infections and antimicrobial use in European acute care hospitals. Stockholm: ECDC; 2013.

46. Azienda USL di Reggio Emilia (a cura di Lorenzani M., Ragni P.). Procedura - Il lavaggio delle mani; 2005. Disponibile a: https:/extranet.ausl.re.it/areasanitaria/DipFarm/ CommDipositiviMedici/Procedure/ProcLavMani.pdf

47. McGowan JE Jr. Antimicrobial stewardship - the state of the art in 2011: focus on outcome and methods. Infect Control Hosp Epidemiol 2012;33:331-7.

48. Septimus EJ, Owens RC Jr. Need and potential of antimicrobial stewardship in community hospitals. Clin Infect Dis 2011;53:S8-14

49. Palmay L, Elligsen M, Walker SA, et al. Hospital-wide roll-out of antimicrobial stewardship: a stepped-wedge randomized trial. Clin Infect Dis 2014 [In press].

50. Perez KK, Olsen RJ, Musick WL, et al. Integrating rapid diagnostics and antimicrobial stewardship improves outcomes in patients with antibiotic-resistant Gram-negative bacteremia. J Infect 2014 [In press].

51. Aiken LH, Sloane DM, Bruyneel L, et al. Nurse staffing and education and hospital mortality in nine European countries: a retrospective observational study. Lancet 2014;383:1824-30.

52. Palese A, Watson R. Nurse staffing and education in Europe: if not now, when? Lancet 2014;383:1789-90.

53. Bell CM, Redelmeier DA. Mortality among patients admitted to hospitals on weekends as compared with weekdays. N Engl J Med 2001;345:663-8.

54. Handel AE, Patel SV, Skingsley A, et al. Weekend admissions as an independent predictor of mortality: an analysis of Scottish hospital admissions. BMJ Open 2012;2:6.

55. Gardellini A, Nardi R, Arienti V, et al. Aging, patientbed management and overcrowding in the medical departments. Ital J Med 2009;3:35-43.

56. Nardi R, Gardellini A, Iori I. Internal Medicine wards overcrowding and clinical risk management: structural or systemic interventions needed? Ital J Med 2009;3:3-8.

57. Kaier K, Mutters NT, Frank U. Bed occupancy rates and hospital-acquired infections--should beds be kept empty? Clin Microbiol Infect 2012;18:941-5.

58. Kulstad EB, Sikka R, Sweis RT, et al. ED overcrowding is associated with an increased frequency of medication errors. Am J Emerg Med 2010;28:304-9.

59. Lovato E, Minniti D, Giacometti M, et al. Il sovraffollamento nel dipartimento di emergenza: efficacia di una strategia multifattoriale isorisorse Evidence 2013;5: e1000056.

60. Tarnow-Mordi WO, Hau C, Warden A, Shearer AJ. Hospital mortality in relation to staff workload: a 4-year study in an adult intensive-care unit. Lancet 2000;356: 185-9.

61. Sprivulis PC, Da Silva J-A, Jacobs IG, et al. The association between hospital overcrowding and mortality among patients admitted via Western Australian emergency departments. Med J Aust 2006;184:208.

62. Borg MA, Suda D, Scicluna E. Time-series analysis of the impact of bed occupancy rates on the incidence of Staphylococcus aureus infection in overcrowded general wards. Infect Control Hosp Epidemiol 2008;29:496-502.

63. Elliott DJ, Young RS, Brice J, et al. Effect of hospitalist workload on the quality and efficiency of care. JAMA Intern Med 2014;174:786-93.

64. Dall'Orto F, Desimoni G, Scalabrini E, et al. Brief internistics observation: what diseases are admitted and what outcome? Ital J Med 2014;8:33-4.

65. Serafini F, Bonanni L, Zancanaro A, et al. Area of admission and short-term care: an integral part of the internal medicine ward organized to intensive care. Ital J Med 2012;6:242-7.

66. Matarrese D. Il territorio in divenire verso l'avvenire. Relazione al convegno "Esperienze di cure primarie: l' innovazione 2.0 nell'assistenza territoriale", Bologna, 31 Marzo-1 Aprile 2014. Disponibile a: http://www.saluter.it/documentazione/convegni-e-seminari/cure-primarie-2.0-bologna-2014/Matarrese_Toscana.pdf

67. Renwick M, Gillett S, Liu Z. Long-stay older patients in acute hospitals: are they bed blockers? Aust Health Rev 1992;15:284-98.

68. Carter J, Mayhew J. Audit of hospital transfers January to March 2006 from Sussex police custody. J Forensic Leg Med 2010;17:38-40.

69. Eherenwerth J, Sorbo S, Hackel A. Transport of critically ill adults. Crit care Med 1986;14:543-7.

70. Ministero della Salute, Dipartimento della Qualità Direzione Generale della Programmazione Sanitaria, dei Livelli di assistenza e dei principi di etica di sistema. Ufficio III $^{\circ}$ Raccomandazione ${ }^{\circ} 11$, Governo Clinico e Sicurezza dei pazienti; Gennaio 2010.

71. Gruppo di Studio SIAARTI Emergenza. Raccomandazioni per $\mathrm{i}$ trasferimenti inter ed intra ospedalieri SIAARTI, 31 ottobre 2012 Prot. n. 562/b Documento approvato dal Consiglio Direttivo S.I.A.A.R.T.I. - Napoli, 24 ottobre 2012 . 


\title{
Nuovi assetti organizzativi in area medica: aspetti gestionali clinici ed economici
}

\author{
Ilario Stefani, Antonino Mazzone \\ Dipartimento di area Medica, Azienda Ospedaliera di Legnano (MI), Italia
}

\begin{abstract}
RIASSUNTO
La medicina moderna si trova oggi ad affrontare l'esigenza di garantire continuità nella cura e nella conoscenza dell'ammalato e, contemporaneamente, quella di operare in contesti ad alto contenuto tecnologico e ad alta specializzazione. Queste novità stanno determinando profonde conseguenze nell'ambito delle discipline mediche, introducendo metodologie di lavoro improntate alla multidisciplinarietà, all'integrazione, all'implementazione, al lavoro contestualizzato. La maggior parte delle patologie croniche richiede l'integrazione di competenze diverse, in parte non mediche, in parte non sanitarie. Il requisito della comunicazione diviene quindi determinante e dominante ai fini del conseguimento della qualità del servizio e del rapporto con gli utenti. Nella definizione dei modelli di funzionamento degli ospedali e di quelli d'integrazione fra ospedale e territorio incontriamo la contraddizione per cui la maggior parte della competenza medica risiede nell'ospedale ed è poco disponibile a fornire prestazioni all'esterno in un contesto di bassa intensità di cura, mentre la domanda di cure nasce prevalentemente sul territorio e richiede in modo limitato, ma preciso, l'accesso alla competenza medica e alla tecnologia ospedaliera. Il problema centrale è quello di definire processi di servizio clinico-assistenziali in cui l'obiettivo della conservazione di uno stato sufficiente/adeguato di salute e autosufficienza sociale sia affidato a un gestore del processo che abbia accesso all'ottenimento di tutte le prestazioni occorrenti al conseguimento del risultato. Le esperienze della assistenza domiciliare integrata per il trattamento delle malattie e dei problemi sociali dell'anziano offrono spunti di riflessione interessanti per comprendere a quali modelli di servizio sarà opportuno riferirsi in futuro. È in tale contesto che L'Azienda Ospedaliera Ospedale Civile di Legnano ha sviluppato un nuovo modello organizzativo per il raccordo fra la cura della fase acuta della malattia e il reinserimento nell'ambiente quotidiano di vita, con prolungati interventi a supporto di una crescente fragilità, favorendo l'integrazione dei servizi e il lavoro di rete.
\end{abstract}

\section{Introduzione}

L'organizzazione può essere definita come un sistema che elabora informazioni, risolve problemi, si esprime con la capacità di trasformare le informazioni che provengono dai suoi componenti e dai suoi clienti per prendere decisioni e dare risposte e risultati appropriati al contesto. ${ }^{1}$ Questo elemento è particolarmente importante in sanità, dove abbiamo assistito negli ultimi decenni a una modificazione del concetto di salute, dell'attesa di salute e di benessere da parte del pubblico.

Corrispondente: Antonino Mazzone, Dipartimento Area Medica, AO di Legnano, via Papa Giovanni Paolo II 1, 20025 Legnano (MI), Italia.

E-mail: medicina2legnano@ao-legnano.it

Parole chiave: organizzazione dipartimentale, continuità di cura, integrazione ospedale-territorio, assistenza domiciliare, lavoro in rete.

Articolo pubblicato secondo la Creative Commons Attribution NonCommercial 3.0 License (CC BY-NC 3.0).

(C) Copyright I. Stefani e A. Mazzone, 2014

Licensee PAGEPress, Italy

QUADERNI - Italian Journal of Medicine 2014; 2:125-130
L'invecchiamento della popolazione, infatti, e il conseguente incremento dell'età media hanno aumentato il numero di soggetti affetti da patologie croniche, da patologie multiple e da patologie complesse.

Nell'ultimo decennio il Sistema Sanitario Italiano è stato oggetto di profonde modifiche volte a sviluppare l'efficienza produttiva, l'efficacia e l'appropriatezza dei servizi sanitari attraverso l'introduzione di nuovo assetti organizzativi delle Unità Operative (UO) e di modelli di tipo dipartimentale, promossi in particolare dal D.lgs. 502/92. Recentemente gli studi si sono concentrati sulla possibilità di realizzare il Governo Clinico attraverso il dipartimento, considerato il contesto organizzativo più adeguato per l'implementazione dello stesso. La clinical governance si sviluppa come prospettiva unificante degli aspetti clinici ed economici nelle prestazioni sanitarie, strumento attraverso il quale le organizzazioni del sistema sanitario nazionale possono migliorare continuamente la qualità delle cure, focalizzandosi sull'eccellenza clinica derivante dall'adozione di elevati standard di cura.

Diviene necessario affrontare cambiamenti strutturali importanti, sia sul versante dell'offerta, dove l'innovazione tecnico-scientifica produce possibilità impensabili fino a pochi anni fa, sia sul versante della domanda, dove la composizione demografica è molto variata rispetto a poco tempo fa. Il prevalere della ge- 
stione dell'acuzie e dello sviluppo dell'efficienza delle strutture di offerta ospedaliera può ora divenire paradossalmente ostacolo nella nuova stagione caratterizzata dalla cronicità e insieme dalla necessità di governare la domanda con un'organizzazione diffusa su scala territoriale. ${ }^{2}$

Gli scopi della Medicina si sono pertanto ridefiniti in rapporto alla conoscenza della malattia, alla terapia e alla relazione con il paziente, ma anche alla conoscenza gestionale ed economica dell'attività clinica, all'utilizzo razionale delle risorse messe a disposizione dalle Politiche Sanitarie.

Questi concetti stanno determinando profonde conseguenze nell'ambito delle discipline mediche, introducendo metodologie di lavoro improntate alla multidisciplinarietà, all'integrazione, all'implementazione, al lavoro contestualizzato.,

La Medicina Interna gestisce la diagnosi e la terapia dei pazienti complessi, poli-patologici, fragili, permettendo un utilizzo appropriato delle funzioni specialistiche ad alta tecnologia; ha la capacità di rispondere alle fasi più critiche del processo di assistenza, facendosi carico del coordinamento delle cure multi-professionali. La complessità nella gestione dell'assistenza può quindi essere sostenuta dal Medico Internista, capace di svolgere un'azione di coordinamento, d'integrazione, di cooperazione fra le diverse parti che agiscono per ambiti specifici di specializzazione settoriale. In relazione alle caratteristiche di ogni paziente l'UO di Medicina Interna, in una logica di appropriatezza, promozione della qualità e di efficacia dell'assistenza, dovrebbe essere in grado di offrire uno specifico percorso assistenziale, del quale il tradizionale ricovero ospedaliero costituisce solamente una delle possibilità o una sua parte. ${ }^{5}$

L'obiettivo di analisi efficace ed efficiente del paziente complesso non può prescindere da una riorganizzazione della realtà sanitaria. Questa, ancora incentrata sul ricovero ospedaliero, volto alla cura della singola malattia acuta, e su indicatori di costo basati sul sistema dei diagnosis-related group, non sembra ormai sufficiente per gestire l'impatto economico nell'ambito della complessità, soprattutto in un momento in cui le risorse erogabili in campo sanitario sono limitate. I costi richiesti per la gestione di un malato complesso sono superiori alla somma delle spese per singola patologia. Inoltre, dal punto di vista della programmazione sanitaria, un'inadeguata valutazione della complessità e il mancato coordinamento tra le varie specialità possono portare a ritardi diagnostico-terapeutici, riammissioni improprie in ospedale, frammentazioni e duplicazioni terapeutiche con allocazione delle risorse inappropriata.

\section{Modello organizzativo per intensità di cura}

I pazienti ricoverati in urgenza presso un'UO di Medicina Interna possono presentare condizioni clini- che differenti e pertanto necessitare di una tipologia diversa di assistenza di tipo medico e infermieristico. Per rispondere a questa variabilità di presentazione si sono affermati modelli organizzativo-assistenziali che tendono a superare il modello statico di Divisione ospedaliera, suddiviso per specializzazione e caratterizzato da un'omogenea distribuzione di pazienti, indipendentemente dalla loro situazione patologica. Il modello assistenziale basato sull'intensità delle cure può essere costruito come modello organizzativo di UO (in particolare per le realtà più decentrate ove all'interno delle UUOO di Medicina Interna trovano posto settori specialistici e mancano spesso aree intensive specialistiche dedicate) o come modello organizzativo dipartimentale o interdipartimentale (specie negli ospedali di grandi dimensioni dove possono essere strutturate aree di degenza omogenea cui afferiscono pazienti di più unità operative che vi sono allocati e distribuiti secondo le necessità assistenziali.

Il rafforzamento d'interazione, collaborazione polispecialistica e multidisciplinare, e della sinergia tra competenze e professionalità è imposto dal fatto che anche la maggior parte dei pazienti acuti è in genere caratterizzata da pluripatologie e che le cure tramite farmaci e metodiche avanzate sono caratterizzate da grande efficacia, ma, al tempo stesso, possono determinare rilevanti effetti collaterali non sempre considerati da chi ha un approccio specialistico.

La considerazione unitaria del paziente, per la diagnosi e la cura, tradizionalmente è stata affidata alla figura dell'internista, dato l'enorme progresso delle conoscenze è molto difficile che tale funzione possa essere svolta da un unico medico, che non sia un internista qualificato da grande esperienza.

Si ritiene perciò maggiormente realistico pensare a un'organizzazione propria di strutture a elevata complessità e non più di tipo artigianale, nella quale la completa responsabilità e la potestà dell'indicazione diagnostica e terapeutica sia affidata a unità/equipe mediche polispecialistiche, dirette da un medico dotato di elevata esperienza, competenza, autorevolezza e di efficaci strumenti organizzativi (primario, internista). Ognuna di esse sarà composta di medici con competenze complementari definite sulla base delle caratteristiche prevalenti dei pazienti, della dotazione di personale e di modelli organizzativi che ogni ospedale adotta in ragione della propria storia e delle forme di collaborazione consolidate e condivise. A tali unità è attribuita la funzione di organizzare al meglio le cure e il loro iter, liberate da incombenze burocratiche eccessive e dai carichi di gestione di settori o persone, che sono organizzate e gestite da altre figure professionali che forniscono servizi alle unità/equipe mediche secondo le loro richieste e indicazioni. Ogni unità/equipe medica si organizza internamente per gestire le patologie multiple attraverso accordi e/o inse- 
rimenti di competenze anche di discipline diverse da quella specifica (ad es. inserimento di competenze internistiche, nei reparti chirurgici quali Ortopedia, Chirurgia vascolare, ecc.)

In questo modello organizzativo si possono identificare aree di degenza a differenti livelli assistenziali

\section{Area ad alta intensità di cura}

Dedicata a pazienti affetti da patologie acute gravi con alterazione di una o più delle funzioni vitali e che necessitano di monitoraggio di tali parametri e di terapie complesse. Coinvolge pazienti che, per età, comorbilità, caratteristiche generali, problemi logistici non sono eleggibili per ricovero presso unità di terapia intensiva o sub intensiva di tipo specialistico.

La gestione di tale area prevede una norma di lavoro di tipo multidisciplinare. Devono essere definiti protocolli e percorsi per l'accesso, il coinvolgimento di specialisti di altre unità operative e percorsi di dimissione o di trasferimento presso le altre aree di degenza o presso altre unità operative. In queste aree è di particolare rilievo il ruolo del personale infermieristico, specificamente addestrato all'utilizzo dei sistemi di monitoraggio così come all'utilizzo dei principali presidi terapeutici secondo protocolli condivisi e predefiniti.

\section{Area per pazienti acuti}

Dedicata a pazienti affetti da patologie acute potenzialmente a rischio di evoluzione negativa se non trattati in maniera adeguata. Si tratta di pazienti che presentano una condizione di relativa stabilità clinica, spesso anziani nei quali la coesistenza di più patologie croniche con l'acuzie motivo del ricovero pone indicazione a ricovero ospedaliero. Pur necessitando di adeguata sorveglianza e controllo evolutivo, non presentano indicazioni a un monitoraggio in continuo delle principali funzioni vitali.

\section{Area a bassa intensità di cura}

Dedicata al paziente stabilizzato, ma ancora a potenziale elevato rischio di ricadute o complicanze con necessità necessitante di terapia ospedaliera complessa. I dati della letteratura indicano che la popolazione in ricovero ordinario in Medicina comprende circa un quinto di soggetti gestibili in quest'area, non necessitanti di monitoraggio clinico intensivo ma di controlli medici ravvicinati e sostegno infermieristico per garantire le funzioni d'organo, l'espletamento delle attività quotidiane e la riattivazione funzionale.

L'organizzazione ospedaliera in unita operative, favorita dalla crescita delle competenze specialistiche, ha introdotto rilevanti elementi di frammentazione nel processo di cura, con conseguenze sul piano dell'efficacia dell'intervento e dei costi sostenuti. Nell'attuale visione sistemica diviene necessario recuperare spazi di integrazione e coordinamento, per promuovere una continuità assistenziale di tipo multidisciplinare che sappia preservi le varie linee di specializzazione.

La scelta del modello dipartimentale sembra ben rispondere a questa esigenza, modulando l'assetto organizzativo ospedaliero tramite l'accorpamento di unità operative correlate sul piano clinico. Nella dimensione di area di degenza dipartimentale, considerando un maggior numero sia di posti letto sia di specialisti interessati, servono modifiche organizzative correlate al grado di complessità, sostenendo la condivisione di risorse umane, fisiche e di know-how secondo la prospettiva della clinical governance.

La missione del dipartimento di Area Medica è di trattare patologie di tipo acuto, organizzando l'assistenza attorno all'idea della cura secondo il modello dell'intensità. I nuovi modelli organizzativi rendono necessaria l'introduzione di figure nuove, la principale delle quali è il responsabile del caso (Tutor), ruolo di riferimento sia per il personale che lavora nell'area sia per il paziente sia. Considerando la specificità del medico Internista nel gestire la complessità appare verosimile che la maggior parte della casistica sia gestita da tale figura; se nel dipartimento sono presenti altre specialità di area i medici di relativa appartenenza gestiranno direttamente i casi che richiedono particolari competenze, fornendo il loro prezioso e costante contributo come consulenti (Consultant) per tutte le altre situazioni.

Nei presidi ospedalieri con numero di posti letto limitato la realizzazione dei dipartimenti di area funzionale trova un limite nella scarsità delle discipline che qui in genere operano. In tali contesti si può immaginare una sola struttura dipartimentale (dipartimento di presidio), con aree di degenza, sempre organizzate secondo il modello dell'assistenza per intensità di cura, di tipo medico-chirurgico, ove il medico Internista continui a svolgere la funzione di principale gestore del paziente.

L'approccio di sistema, una volta raggiunta la comprensione del malato complesso, deve proporsi anche di elaborare una proposta di riorganizzazione e pianificazione dell'assistenza sanitaria in fase di dimissione dall'ambito per acuti, che sia in grado di gestire quadri clinici di elevata complessità. Diviene, infatti, prioritario, una volta identificati i determinanti della complessità, proporre nuove strategie di ricerca che includano $\mathrm{i}$ quadri di alta complessità clinica. È necessario definire e attuare percorsi diagnostici e assistenziali che possano permettere una gestione coordinata, multi-professionale e a lungo termine del paziente complesso tali da garantire una continuità assistenziale ospedale-territorio, in 
modo che il territorio possa farsi carico dell'accoglienza del paziente dopo un'ospedalizzazione e delle strategie di prevenzione secondaria.

La nuova cultura sistemica vede il Distretto e l'Ospedale come componenti complesse di un continuo indissolubile sul tema dell'appropriatezza come risposta ai fabbisogni assistenziali. Il concetto di appropriatezza, infatti, non va applicato soltanto alle attività clinico-assistenziali ma anche al luogo (setting). L'appropriatezza del luogo di cura e del professionista più pertinente (non solo in termini di competenze ma anche di tecnologie e di tempi disponibili) per i fabbisogni del paziente è un prerequisito di economicità $\mathrm{e}$ di qualità complessiva sia dell'offerta sia della relazione servizio-utente.

La gestione del paziente in dimissione è essenziale per garantire un'appropriata prosecuzione dell'assistenza sul territorio. L'organizzazione di questa fase del processo assistenziale richiede l'identificazione del bisogno di cura della persona alla dimissione, la complessità dei suoi bisogni socio-assistenziali e verifica i requisiti per il suo soddisfacimento. La regola operativa prevede l'elaborazione di percorsi di dimissione protetta che contemplino il bisogno di cura le problematiche collegate a nuove disabilità o a situazioni logistico familiari (persona che vive sola, as- senza di caregiver). Esistono inoltre situazioni cliniche che necessitano di una prosecuzione di monitoraggio [patologie ad andamento cronico quali bronco pneumopatia cronica ostruttiva (BPCO), scompenso cardiaco, ecc.] con percorsi clinico assistenziali integrati ospedale-territorio, coinvolgendo il MMG. In quest'ambito le UUOO di Medicina Interna, in collaborazione con i servizi territoriali ed i dipartimenti di cure primarie sono chiamate a svolgere un ruolo fondamentale condividendo appositi percorsi atti a gestire e trattare l'ambito della cronicità.

In quest'ottica abbiamo intrapreso una nuova modalità organizzativa creando, per questi pazienti, percorsi di raccordo fra la cura della fase acuta della malattia e il reinserimento nell'ambiente quotidiano di vita, con un intervento mirato in grado di consentire l'integrazione dei servizi sanitari, sociali e assistenziali, con la costituzione di un Ambulatorio e call center di continuità assistenziale ospedale - territorio polispecialistico di area medica (Figura 1), dove si attiva, nella presa in carico del paziente fragile, una rete tra l'assistenza domiciliare integrata (ADI-voucher), il MMG e l'Ospedale, implementando un modello organizzativo di case management. ${ }^{6} \mathrm{Al}$ paziente è offerta per un periodo di trenta giorni dalla dimissione, eventualmente proseguibile secondo le necessità cliniche, in affianca-

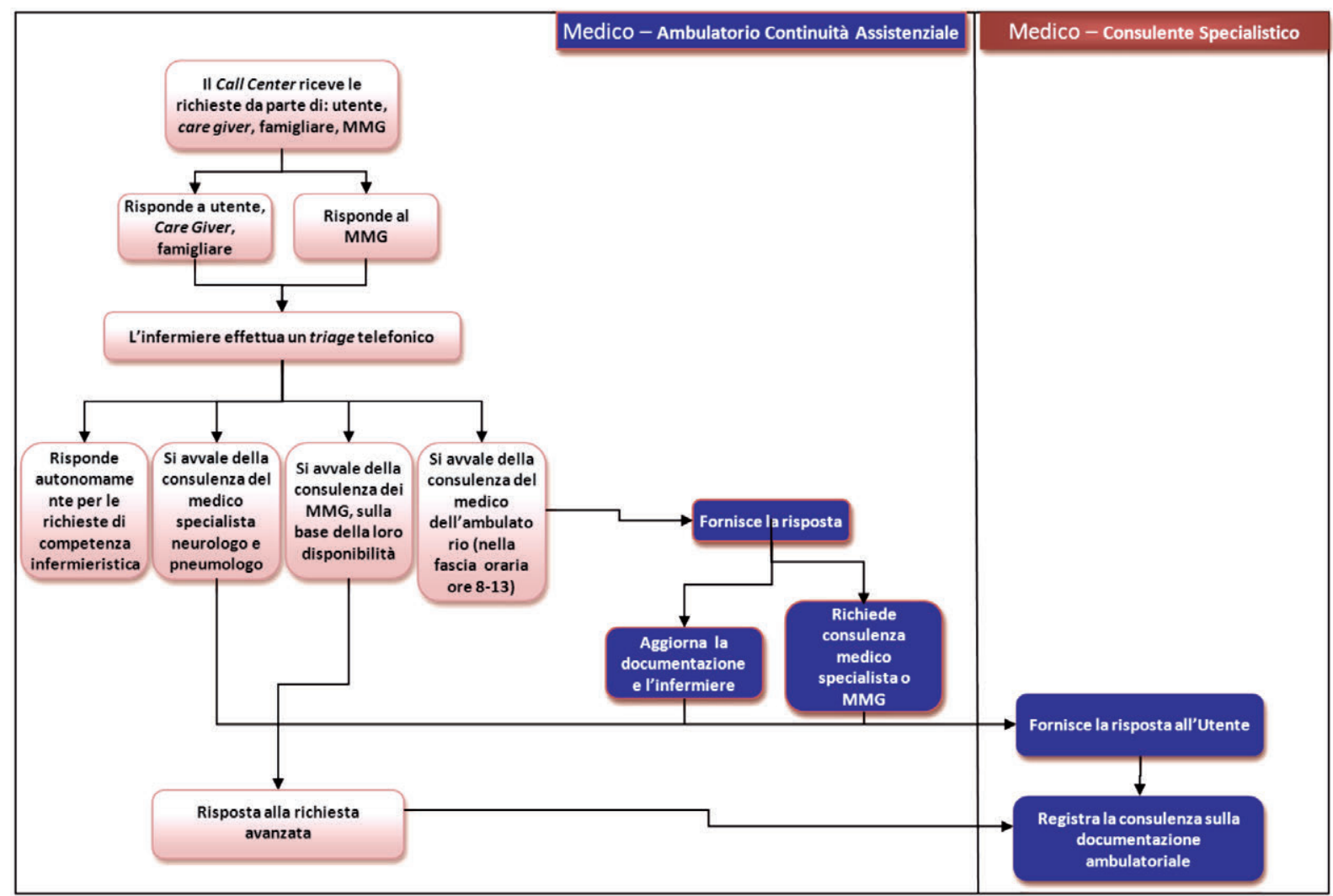

Figura 1. Ambulatorio e call center di continuità assistenziale ospedale - territorio polispecialistico di area medica. 
mento all'assistenza del MMG e dell'ADI-voucher con pattanti della rete socio-sanitaria, l'assistenza specialistica ospedaliera attraverso il call center e l'ambulatorio dedicato di area medica. La popolazione di pazienti arruolata prevede l'inclusione dei malati che, a seguito di patologie acute cerebrovascolari e cardiovascolari, abbiano postumi invalidanti e/o patologie croniche ingravescenti, ricoverati presso le degenze ospedaliere delle UUOO del Dipartimento Medico. L'attività clinico-assistenziale è stata sviluppata implementando un organizzativo di case management attraverso: i) presenza del case manager infermieristico (due infermieri professionali dedicati); ii) presenza di responsabile medico (un dirigente medico di area internistica); iii) presenza di un piano assistenziale personalizzato predisposto in multi-professionalità e multi-disciplinarietà; iv) individuazione delle modalità di attivazione e integrazione dei Servizi del Sistema Socio Sanitario; coinvolgimento dei MMG e assistenza domiciliare dell'ASL; v) elaborazione e applicazione dei percorsi diagnostici terapeutici assistenziali condivisi fra ospedale e territorio.

Partendo dalla considerazione che le ammissioni con medesima diagnosi entro i 30 giorni dalla prima dimissione sono considerati dal Servizio Sanitario Nazionale come impropri e pertanto non sono rimborsati, tale sperimentazione ha dimostrato la possibilità di una riduzione di tale tipologia di prestazioni per l'A- zienda Ospedaliera di riferimento. Proprio in relazione a tale diminuzione, i risultati di efficacia ottenuti mediante la sperimentazione dell'ambulatorio call center si sono rivelati significativamente superiori rispetto a simili dati derivanti da analisi italiane e internazionali, relative a scompenso cardiaco e altre patologie di riferimento (insufficienza respiratoria, BPCO), evidenziando una percentuale di re-ricoveri entro i 30 giorni, per medesima diagnosi sul totale dei pazienti presi in carico, pari al 6\% (Tabella 1). Due studi condotti in differenti Regioni italiane, infatti, riportano un tasso di riammissione per insufficienza cardiaca in pazienti non gestiti pari rispettivamente al $15 \%{ }^{7}$ e al $17,4 \%{ }^{8}$ Altri studi di stampo internazionali mostrano un tasso semestrale di rientro in ospedale pari al $18 \%$ nei Paesi Bassi ${ }^{9}$ e al $49 \%$ in Canada. ${ }^{10}$ In una scala di valutazione da $1 \mathrm{a} 7$, è stato rilevato un punteggio medio di valutazione complessiva pari a 6,31 , con un livello di soddisfazione di pazienti/familiari/caregiver pari a 6,50 , per quanto riguarda le informazioni (Figura 2).

Una possibile ipotesi di lavoro per una prima valutazione economica è rappresentata anzitutto dalla quantificazione dei costi del personale coinvolto nell'attuazione del progetto: due infermieri professionali (fabbisogno per monte ore pari a 60 ore settimanali) e di un dirigente medico (fabbisogno stimato di 20 ore settimanali) per un costo annuale del personale pari a

Tabella 1. Percentuale di re-ricoveri nel periodo 01/09/2010 - 19/03/2014.

Percentuale di re-ricoveri entro 30 giorni con medesima diagnosi sul totale dei pazienti presi in carico per scompenso cardiaco/insufficienza respiratoria/BPCO

Percentuale di re-ricoveri entro 90 giorni con medesima diagnosi sul totale dei pazienti presi in carico per scompenso cardiaco/insufficienza respiratoria/BPCO

Fonte: Azienda Ospedale Civile Legnano (2013). BPCO, bronco pneumopatia cronica ostruttiva.

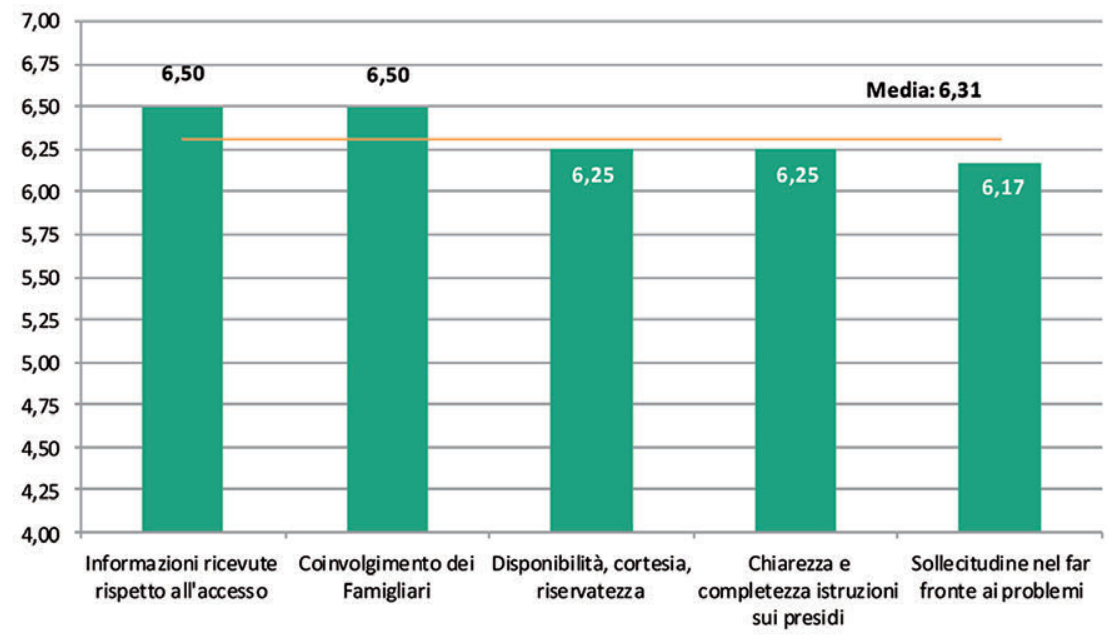

Figura 2. Analisi customer satisfaction (scala di gradimento crescente da 1 a 7). Fonte: Azienda Ospedale Civile Legnano (2013). 
Tabella 2. Valutazione economica.

\begin{tabular}{ll}
\hline $\begin{array}{l}\text { Insufficienza cardiaca (428.0) } \\
\text { (rimborso medio regionale 5865 euro) }\end{array}$ & $\begin{array}{l}\text { Insufficienza cardiaca sinistra (codice 428 } \\
\text { (rimborso medio regionale 5788 euro) }\end{array}$ \\
\hline $\begin{array}{l}\text { Insufficienza respiratoria (codice 518.81) } \\
\text { (rimborso medio regionale 5404 euro) }\end{array}$ & $\begin{array}{l}\text { BPCO riacutizzata (codice 421.91) } \\
\text { (rimborso medio regionale 3214 euro) }\end{array}$ \\
\hline
\end{tabular}

Con una media di rimborso per il case-mix considerato di 5067,75 euro/caso

100.000 euro/anno. Se stimiamo per i re-ricoveri con medesima diagnosi a 30 giorni nell'ambito delle categorie diagnostiche considerate nel progetto insufficienza cardiaca (428.0) (rimborso medio regionale 5865 euro); insufficienza cardiaca sinistra (codice 428.1) (rimborso medio regionale 5788 euro); insufficienza respiratoria (codice 518.81) (rimborso medio regionale 5404 euro); BPCO riacutizzata (codice 421.91) (rimborso medio regionale 3214 euro); con una media di rimborso per il case-mix considerato di 5067,75 euro una riduzione del $22 \%$ (28\% stimato dai dati storici aziendali - $6 \%$ ottenuto come risultato dell'attività in oggetto) per un totale di 75,9 casi $(22 \%$ dei 345 pazienti con tali diagnosi di dimissione stimati dai dati di attività della nostra UO possiamo approssimativamente stimare un risparmio di 384.642,22 euro/anno (Tabella 2).

Nella definizione dei modelli di funzionamento degli ospedali e di quelli d'integrazione fra ospedale e territorio incontriamo la contraddizione per cui la maggior parte della competenza medica risiede nell'ospedale ed è poco disponibile a fornire prestazioni all'esterno in un contesto di bassa intensità di cura, mentre la domanda di cure nasce prevalentemente sul territorio e richiede in modo limitato, ma preciso, l'accesso alla competenza medica e alla tecnologia ospedaliera. Il problema centrale è quello di definire processi di servizio clinico-assistenziali in cui l'obiettivo della conservazione di uno stato sufficiente/adeguato di salute e autosufficienza sociale sia affidato a un gestore del processo che abbia accesso all'ottenimento di tutte le prestazioni occorrenti al conseguimento del risultato. La nostra esperienza conferma l'importanza di una nuova visione organizzativa sistemica per la gestione e l'appropriato utilizzo dei posti letto in ambito ospedaliero, in particolare per le malattie croniche. ${ }^{11}$

\section{Bibliografia}

1. Micossi P. Il ruolo dell'ospedale del futuro. In: Falcitelli N, Trabucchi M, Vanara F (a cura di). Rapporto Sanità 2000: documento introduttivo; Rapporto Sanità 2000. L'ospedale del futuro. Bologna: il Mulino; 2000. p 428.

2. Regione Lombardia. Libro Bianco sulla Sanità Regionale. Milano: Regione Lombardia Eds; Luglio 2014.

3. Federspil G. I fondamenti del metodo in medicina clinica e sperimentale. Padova: Piccin Ed.; 1980.

4. Scandellari C. La strategia della diagnosi. Padova: Piccin Ed.; 1981.

5. Nardi R, Scanelli G, Borioni D, et al., The assessment of complexity in internal medicine patients. The FADOI Medicomplex Study. Eur J Int Med 2007;18:283-7.

6. Stefani I, Poesio C, Calloni MV, et al. Sperimentazioni organizzative in medicina interna: l'ambulatorio Call Center. Sanità Pubblica e Privata. Riv Dir Econ Manag Sanità 2011;6:34-42.

7. Valle R, Carbonieri E, Tenderini P, et al. Una proposta di protocollo per la gestione ambulatoriale dei pazienti dimessi con diagnosi di scompenso cardiaco: il progetto collaborativo Venetia - HF. In Ital Heart J 2004;5:282-91.

8. Anselmino M, Petrelli A, Gnavi R, et al. Epidemiologia dei ricoveri per scompenso cardiaco in Piemonte tra il 1996 e il 2001. Ital Heart J 2005;6:42-52.

9. Reitsma R, Mosterd A, de Craen AJ, et al. Increase in hospital admission rates for heart failure in The Netherlands, 1980-1993. In Heart 1996;79:388-92.

10. Feldman DE, Thivierge C, Guerard L, et al. Changing trends in mortality and admissions to hospital for elderly patients with congestive heart failure in Montreal. CMAJ 2001;165:1033-6.

11. Stefani I. Comment to "Joynt KE, Jha AK. Thirty-Day Readmissions-Truth and Consequences. N Engl J Med 2012;366:1366-9.” Disponibile a: http://www.salute.gov. it/portale/temi/documenti/dispositiviMedici/conferenza_3 995/35.pdf 


\title{
Troppe procedure
}

\author{
Marco Bobbio, ${ }^{1}$ Luigi Lusiani, ${ }^{2}$ Roberto Frediani ${ }^{3}$ \\ ${ }^{1} \mathrm{SC}$ Cardiologia, Ospedale Santa Croce e Carle, Cuneo; ${ }^{2} \mathrm{SC}$ Medicina Interna, Castelfranco Veneto (TV); ${ }^{3}$ Direttore SC Medicina \\ Interna, Verbania e Domodossola (VB), Italia
}

\section{RIASSUNTO}

In cardiologia sono stati documentati molti casi riguardanti l'ampia diffusione di procedure diagnostiche e terapeutiche, nonostante la scarsità di prove di efficacia (dissociazione procedure-esiti), dal momento che nella pratica clinica vengono adottate procedure che non risultano così efficaci come ci si sarebbe aspettato dai dati di ricerche parziali e dall'esperienza personale. Trentatré anni fa McKinley aveva descritto in 7 tappe la carriera di una nuova procedura: dalla presentazione entusiastica di ricerche-pilota fino all'oblio. I progetti Choosing wisely negli Stati Uniti e Fare di più non significa fare meglio in Italia (a cui hanno aderito l'ANMCO e la FADOI) si pongono l'obiettivo di individuare i test diagnostici e i trattamenti prescritti molto comunemente, costosi, con un certo grado di rischio e che, secondo prove scientifiche di efficacia, non apportano benefici significativi alle principali categorie di pazienti ai quali venivano comunemente prescritti. Nell'articolo viene infine descritta l'applicazione di un progetto per individuare pratiche a rischio di inappropriatezza in un ospedale italiano.

\section{Introduzione}

Undici anni fa, quando vennero pubblicati i risultati dello studio TEMISTOCLE${ }^{1}$ (una ricerca collaborativa ANMCO-FADOI sui pazienti ricoverati per scompenso cardiaco in reparti di cardiologia e di medicina interna) molti cardiologi e internisti si stupirono osservando che, a fronte di una maggior prescrizione ai pazienti ricoverati nei reparti di cardiologia rispetto a quelli di medicina interna di ecocardiogrammi $(89,3$ vs $54,8 \%$; $\mathrm{P}<0,001)$, di monitoraggi Holter $(29,0 \%$ vs $8,0 \%$; $\mathrm{P}<0,001)$ e di coronarografie $(7,5$ vs $0,9 \% \mathrm{P}<0,001)$, dopo 6 mesi risultava sovrapponibile il tasso di rericoveri $(43,7$ vs 45,$4 ; \mathrm{P}=\mathrm{NS})$ e della mortalità $(13,9 \%$ vs $16,7 \% ; \mathrm{P}=\mathrm{NS})$; tale dato era confermato anche dopo la correzione per variabili di gravità e complessità dei pazienti ricoverati nelle due tipologie di reparti (rischio relativo 0,97; IC95\% 0,67-1,42). Dunque, un maggior uso di procedure diagnostiche non sembra essere in grado di influire sul successivo decorso clinico

Corrispondente: Marco Bobbio, via Pietra del Gallo 45, 10025 Pino Torinese (TO), Italia.

Tel.: +39.388.6188631.

E-mail:mbobbio51@gmail.com

Parole chiave: inappropriatezza, iperprescrizione, Slow Medicine.

Articolo pubblicato secondo la Creative Commons Attribution NonCommercial 3.0 License (CC BY-NC 3.0).

CCopyright M. Bobbio et al., 2014

Licensee PAGEPress, Italy

QUADERNI - Italian Journal of Medicine 2014; 2:131-134 nonostante una più attenta valutazione strumentale dei pazienti.

Già 10 anni prima i ricercatori, che avevano condotto lo studio $\mathrm{SAVE}^{2}$ (confronto tra captopril e placebo in pazienti con recente infarto miocardico), decisero di utilizzare la casistica per seguire il destino dei 1573 pazienti arruolati negli Stati Uniti e dei 658 arruolati in Canada $^{3}$ e osservarono che la coronarografia era stata eseguita nel $68 \%$ dei primi e nel $31 \%$ dei secondi, una rivascolarizzazione nel $12 \%$ e nel $3 \%$. Avendo constatato che negli Stati Uniti era stato sottoposto a coronarografia il doppio dei pazienti e a procedure di rivascolarizzazioni il quadruplo, i ricercatori si sarebbero aspettati di trovare una sostanziale riduzione di eventi tra i pazienti curati in modo più aggressivo; invece, risultarono uguali la mortalità $(23 \%$ nei pazienti statunitensi e $22 \%$ in quelli canadesi) e l'incidenza di reinfarto (rispettivamente $13 \%$ e $14 \%$ ).

Passò un anno e quei dati vennero confermati da un gruppo di ricercatori che mise a confronto ${ }^{4}$ il destino dei pazienti ricoverati in due analoghi centri universitari, negli Stati Uniti (Stanford University) e in Canada (McGill University). Nel centro statunitense era stato sottoposto a coronarografia il $55 \%$ dei pazienti contro il $34 \%$ in quello canadese, a rivascolarizzazione il $40 \%$ contro il $17 \%$; a fronte di un'incidenza quasi doppia di procedure, dopo 20 mesi la mortalità era del tutto simile (28 contro $27 \%)$. Ad analoghi risultati era giunto un gruppo di ricercatori, analizzando i dati di dimissione di tutti gli infarti cardiaci avvenuti in Belgio dal 1999 al 2001: dopo 5 anni, la mortalità degli infartuati ricoverati in un centro dove veniva eseguita la coronarografia era sovrapponibile a quella che si osservava in un centro in cui non veniva eseguita. ${ }^{5}$ 


\section{Dissociazione procedure-esiti}

Nel corso degli anni sono stati documentati molti altri casi di interesse cardiologico, riguardanti un'ampia diffusione di procedure diagnostiche e terapeutiche, nonostante la scarsità di prove di efficacia: nei pazienti con angina pectoris, una lesione coronarica e segni strumentali di ischemia, l'angioplastica coronarica non sembra offrire maggiori vantaggi rispetto alla terapia medica ottimale ${ }^{6}$ e la disponibilità nell'ospedale del laboratorio di emodinamica aumenta l'incidenza di angioplastiche, ma non riduce il tasso di eventi successivi $;{ }^{7}$ nei pazienti con fibrillazione atriale, con o senza scompenso, il controllo del ritmo cardiaco non riduce l'incidenza di eventi a distanza, rispetto al controllo della frequenza; ${ }^{8,9}$ nei pazienti ischemici con insufficienza mitralica la riparazione della valvola non è più efficace della sua sostituzione; ${ }^{10}$ la denervazione percutanea dell'arteria renale non riduce in modo sostanziale la pressione arteriosa rispetto alla terapia medica, ${ }^{11}$ né riduce l'incidenza di eventi maggiori; ${ }^{12}$ il contropulsatore aortico non riduce la mortalità nei pazienti con infarto complicato da shock cardiogeno, ${ }^{13}$ l'ultrafiltrazione è meno efficace e provoca più effetti indesiderati della terapia diuretica nei pazienti con scompenso cardiaco; ${ }^{14}$ il tasso di infarti non varia nei pazienti con dolore toracico, curati in centri dove vengono svolti molti test diagnostici non invasivi, rispetto a quelli dove se ne usano pochi; ${ }^{15}$ la chiusura percutanea di un forame ovale pervio in pazienti con ictus criptogenetico non riduce in modo significativo la recidiva e la mortalità precoce, ${ }^{16}$ la fibrinolisi nei pazienti embolia polmonare a rischio intermedio riduce lo scompenso emodinamico, ma aumenta il rischio di emorragie maggiori e di ictus; ${ }^{17}$ l'aggiunta di albumina nei pazienti con shock settico non migliora la sopravvivenza a 28 e 90 giorni. $^{18}$

I risultati di queste ricerche non significano che le procedure prese in considerazioni siano inutili, anche perché i dati sono condizionati dal tipo e dalla eterogeneità dei pazienti scelti, dal protocollo adottato, dalla durata del follow-up, dalla numerosità del campione, dalla scelta del gruppo di controllo. Questi articoli ci informano però che le procedure utilizzate abitualmente non sono così efficaci come ci saremmo aspettati dai dati di ricerche parziali e dall'esperienza personale, che tende a sovrastimare l'effetto di ciò che si fa.

\section{La carriera di una procedura}

Come avviene che procedure di non dimostrata efficacia si consolidino nella pratica quotidiana? Già 33 anni fa McKinley ${ }^{19}$ aveva descritto in 7 tappe la carriera di una nuova procedura: la storia inizia con la presentazione entusiastica di ricerche-pilota condotte in centri altamente specializzati, senza aver eseguito confronti con gruppi di controllo e senza criteri di cecità nella valutazione dei risultati; la procedura viene pertanto adottata da altri medici ansiosi di dimostrarsi aggiornati e innovativi. In seguito, la stampa divulgativa si appropria della novità e induce una crescente domanda tra $\mathrm{i}$ pazienti insoddisfatti o ansiosi di ridurre l'incertezza sul loro stato di malattia. Queste fasi sono sostenute da pubblicazioni che inevitabilmente selezionano i risultati positivi (chi mai pubblicherebbe i propri fallimenti?) e da una convincente propaganda dei produttori, cosicché la fama della procedura si consolida fino a diventare uno standard: aumentano $i$ congressi nei quali se ne parla (sempre in modo positivo), le linee guida che la citano (con bassi livelli di evidenza), i centri che la praticano (e se ne vantano), i pazienti che la esigono, le indicazioni cliniche. Quando comincia a sorgere qualche dubbio sull'efficacia, diventa difficile indurre clinici e pazienti, ormai convinti dell'utilità, a eliminare ogni dubbio con un trial randomizzato. Come sostiene McKinley, la storia naturale di alcune procedure finisce talvolta con un definitivo abbandono, come quegli artisti, per lungo tempo esaltati dalla critica, che a un certo punto spariscono nell'oblio. Chi fosse curioso di trovare l'applicazione di queste tappe, si legga l'editoriale di Messerli e Bangalore sulla storia naturale della denervazione dell'arteria renale. ${ }^{20}$

\section{Choosing wisely ${ }^{\circledR}$}

Negli Stati Uniti e nel Regno Unito questa riflessione sull'eccessivo ricorso alle procedure diagnostiche e terapeutiche è in corso da anni. JAMA Internal Medicine ha iniziato a pubblicare una serie di articoli in una nuova rubrica intitolata Less is more, ${ }^{21}$ mettendo in discussione il mito che se un trattamento è buono, fare di più è sempre meglio e analogamente il British Medical Journal ha lanciato la campagna Too much medicine per analizzare cause e rimedi dell'iperprescrizione di metodiche diagnostiche e trattamenti terapeutici. ${ }^{22,23}$

In seguito a un articolo di Howard Brody ${ }^{24}$ nel quale proponeva che ogni società scientifica individuasse una lista di cinque test diagnostici o trattamenti (the top five list) prescritti molto comunemente, costosi, con un certo grado di rischio e che, secondo prove scientifiche di efficacia, non apportassero benefici significativi alle principali categorie di pazienti ai quali venivano comunemente prescritti, l'Advancing Medical Professionalism to Improve Health Care Foundation (ABIM), con la collaborazione della maggiore organizzazione statunitense di consumatori (Consumer Reports) 
lanciò l'iniziativa Choosing wisely ${ }^{\circledR}$, allo scopo di favorire il dialogo tra medici e pazienti in modo da aiutarli a scegliere cure supportate da prove scientifiche, non ridondanti rispetto ad altri test precedentemente effettuati, senza rischi e realmente necessari. ${ }^{25}$ Un primo elenco di 45 test, ad opera di 9 società scientifiche, è stato reso pubblico il 4 Aprile 2012. Ad oggi il progetto ha segnalato oltre 160 tra test e procedure, ad opera di 51 società. Riconoscendo che i pazienti necessitano di migliori informazioni sulle loro cure, Consumer Reports sta sviluppando materiali educazionali e lavorando per disseminarli nella popolazione.

Nel nostro paese il progetto Choosing wisely ${ }^{\circledR}$ è stato adottato dal movimento Slow Medicine ${ }^{26}$ che si è proposto di coordinare un'analoga iniziativa tra le società scientifiche italiane, definendola Fare di più non significa fare meglio. ${ }^{27} \mathrm{Nel}$ progetto italiano, contrariamente a quello condotto negli Stati Uniti, si è deciso di non prendere in considerazione la valutazione dei costi, per non esporre il progetto alla critica di essere mirato a favorire tagli della spesa sanitaria ed evitare una presa di distanza da parte delle associazioni di pazienti, preoccupati che si tratti di una manovra di razionamento e non di razionalizzazione. All'iniziativa hanno aderito l'ANMCO e la FADOI, insieme ad altre 16 società scientifiche nazionali. Il direttivo dell'ANMCO ha costituito un gruppo di lavoro che ha individuato le 5 pratiche, ufficializzate con un position paper, ${ }^{28}$ pubblicato sul Giornale Italiano di Cardiologia.

\section{Fare di più non significa fare meglio all'Ospedale Santa Croce di Cuneo}

L'iniziativa è stata applicata per la prima volta nell'Azienda Ospedaliera Santa Croce e Carle di Cuneo con il progetto aziendale Fare di più non significa fare meglio - Le 3 pratiche a rischio di in appropriatezza con lo scopo di migliorare la qualità $\mathrm{e}$ la sicurezza dei servizi erogati dall'Azienda, attraverso la riduzione di pratiche (esami diagnostici e trattamenti) che, secondo le conoscenze scientifiche disponibili, non apportano benefici significativi ai pazienti ai quali sono generalmente prescritte. Il progetto è riuscito a coinvolgere tutti i reparti, per diventare un patrimonio aziendale condiviso, istituendo un gruppo di lavoro composto con un giovane dirigente per ogni Struttura Complessa. In una prima riunione collegiale è stato illustrato il progetto avviato negli Stati Uniti, ripreso in Italia da Slow Medicine, ed è stato presentato il progetto aziendale, dando mandato di organizzare delle riunioni in ogni Struttura Complessa al fine di individuare le 3 pratiche che si ritiene siano a maggior rischio di inappropriatezza. Nell'arco di un mese sono state svolte riunioni di reparto, condotte con la tecnica del brainstorming, dalle quali è emersa una scheda, discussa collegialmente nel gruppo di lavoro. I risultati sono stati presentati il nell'ambito di un convegno aziendale a cui hanno partecipato i risposabili nazionali di Slow Medicine. Complessivamente sono state individuate tre pratiche da 33 Strutture Complesse, per un totale di 99 pratiche. $^{29}$

\section{Conclusioni}

L'iniziativa testé menzionata, spontanea e partita dal basso (bottom up) in una realtà ospedaliera circoscritta, indica la possibilità di un percorso complementare rispetto a quello iniziato da ABIM negli Stati Uniti $\left(\right.$ Choosing wisely ${ }^{\circledR}$ ) e da Slow Medicine in Italia (Fare di più non significa fare meglio), dove le singole società scientifiche sono uscite allo scoperto, proponendo le proprie raccomandazioni secondo la rispettiva competenza, in maniera necessariamente autoritativa (upside down). Purtuttavia, si tratta di percorsi convergenti e sinergici, che hanno un comune punto di incontro: la appropriatezza prescrittiva secondo i criteri imprescindibili della evidence based medicine e l'attenzione al rapporto efficacia/danno nella pratica clinica.

Una sua estensione alla generalità degli ospedali sarebbe quanto mai auspicabile, e dovrebbe veder in prima linea le Medicine Interne, che più delle altre specialità devono sentirsi chiamate in causa, quando si tratta di riflettere su che cosa voglia dire in concreto orientare la clinica alla salute più che alla malattia, intuendo che una medicina sobria, rispettosa e giusta - oltre che possibile - è comunque la più doverosa.

\section{Bibliografia}

1. Di Lenarda A, Scherillo M, Maggioni AP, et al. Current presentation and management of heart failure in cardiology and internal medicine hospital units: a tale of two worlds. The TEMISTOCLE study. Am Heart J 2003;146:e12.

2. Pfeffer MA, Braunwald E, Moyé LA, et al. Effect of captopril on mortality and morbidity in patients with left ventricular dysfunction after myocardial infarction. Results of the survival and ventricular enlargement trial. The SAVE Investigators. N Engl J Med 1992;327:669-77.

3. Rouleau JL, MoyeLA, Pfeffer MA, et al. A comparison of management patterns after acute myocardial infarction in Canada and the United States. N Engl J Med 1993;328:779-84.

4. Pilote L, Racine N, Hlatky MA. Difference in the treatment of myocardial infarction in the United States and Canada. A comparison of two university hospitals. Arch Intern Med 1994;154:1090-96.

5. Van Brabandt H, Camberlin C, Vrijens F, et al. More is not better in early care of acute myocardial infarction: a 
prospective cohort analysis on administrative databases. Eur Heart J 2006;27:2649-54.

6. Boden WE, O'Rourke RA, Teo KK, et al. Optimal medical therapy with or without PCI for stable coronary disease. N Engl J Med 2007;356:1503-16.

7. Every NR, Larson EB, Litwin PE, et al. The association between on-site cardiac catheterization facilities and the use of coronary angiography after acute myocardial infarction. N Engl J Med 1993; 329:546-51.

8. Wyse DG, Waldo AL, Di Marco JP, et al. A comparison of rate control and rhythm control in patients with atrial fibrillation. N Engl J Med 2002;347:1825-33.

9. Roy D, Talajic M, Nattel S, et al. Rhythm control versus rate control for atrial fibrillation and heart failure. $\mathrm{N}$ Engl J Med 2008;358:2667-77.

10. Acker MA, Parides MK, Perrault LP, et al. Mitral-valve repair versus replacement for severe ischemic mitral regurgitation. N Engl J Med 2014;370:23-32.

11. Bhatt DL, Kandzari DE, O'Neill WW. A controlled trial of renal denervation for resistant hypertension. N Engl J Med 2014;370:1393-401.

12. Cooper CJ, Murphy TP, Cutlip DE, et al. Stenting and medical therapy for atherosclerotic renal-artery stenosis. N Engl J Med 2014;370:13-22.

13. Thiele H, Zeymer U, Neumann FJ, et al. Intraaortic balloon support for myocardial infarction with cardiogenic shock. N Engl J Med 2012;367:1287-96.

14. Bart BA, Goldsmith SR, Lee KL. Ultrafiltration in decompensated heart failure with cardiorenal syndrome. N Engl J Med 2012;367:2296-304.

15. Safavi KC, Li SX, Dharmarajan K, et al. Hospital variation in the use of noninvasive cardiac imaging and its association with downstream testing, interventions, and outcomes. JAMA Intern Med 2014;174:546-53.

16. Carroll JD, Saver JL, Thaler DE, et al. Closure of patent foramen ovale versus medical therapy after cryptogenic stroke. N Engl J Med 2013;368:1092-100.

17. Meyer G, Vicaut E, Danays T, et al. Fibrinolysis for patients with intermediate-risk pulmonary embolism. N Engl J Med 2014;370:1402-11.

18. Caironi P, Tognoni G, Masson S, et al. Albumin replacement in patients with severe sepsis or septic shock. N Engl J Med 2014;370:1412-21.

19. McKinley JB. From "promising reports" to "standard procedure": seven stages of the career of a medical innovation. Milbank Mem Found Q 1981;59:374-411.

20. Messerli FH, Bangalore S. Renal denervation for resistant hypertension? N Engl J Med 2014;370:1454-6.

21. Grady D, Redberg RF. Less is more: how less health care can result in better health. Arch Intern Med 2010;170: 749-50.

22. Godlee F. Too much medicine. BMJ 2013;346:f1328.

23. Davies E. When good care means less pay. BMJ 2013;347:f5997.

24. Brody H. Medicine's ethical responsibil-ity for health care reform. The Top Five list. N Engl J Med 2010;362: 283-5.

25. Richards T, Montori VM, Godlee F, et al. Let the patient revolution begin. Patients can improve healthcare: it's time to take partnership seriously. BMJ 2013;346:f2614.

26. Slow Medicine. Fare di più non significa fare meglio; 2012. Disponibile a: http://slowmedicine.it

27. Hurley R. Can doctors reduce harmful medical overuse worldwide? BMJ 2014;349:g4289.

28. Bobbio M, Abrignani MG, Caldarola $\mathrm{P}$, et al. "Fare di più non significa fare meglio". Le proposte dell'ANMCO. G Ital Cardiol 2014; 15:244-52.

29. Azienda Sanitaria Ospedaliera - S. Croce e Carle, Cuneo. Slow Medicine: progetto "Choosing wisely"; 2014. Disponibile a: http://www.ospedale.cuneo.it/index.php? id $=574$ 


\title{
Medicina Interna e wise medicine: le proposte FADOI, le prospettive assistenziali e le ricadute gestionali
}

\author{
Grazia Panigada, ${ }^{1}$ Irene Chiti, ${ }^{1}$ Alberto Fortini, ${ }^{2}$ Luca Masotti, ${ }^{3}$ Niccolò Napoli ${ }^{4}$ \\ ${ }^{1}$ UOC Medicina Interna, Ospedale SS Cosma e Damiano Pescia, Azienda USL3, Pistoia; ${ }^{2}$ UOC Medicina Interna, Ospedale \\ San Giovanni di Dio, AS Firenze, ASF-Firenze; ${ }^{3}$ UOC Medicina Interna, Ospedale Santa Maria Nuova, AS Firenze, ASF- \\ Firenze; ${ }^{4}$ Scuola di Specializzazione in Chirurgia Generale Università di Pisa, UOC Chirurgia generale e trapianti nell'uremico \\ e nel diabetico, AOU Pisana, AOUP-Pisa, Italia
}

\begin{abstract}
RIASSUNTO
L'agire secondo i principi di appropriatezza, efficacia e sostenibilità, rappresenta l'impostazione corretta per un management efficace ed efficiente delle principali patologie soprattutto in ambito internistico con pazienti polipatologici e complessi. È fondamentale far crescere tra gli internisti un ragionamento critico alla luce di conoscenze e evidenze su test e procedure mediche che possono non essere necessarie o addirittura dannose, stimolandolo ad evitare quelle decisioni che non porterebbero tangibili vantaggi per il paziente. Necessario coinvolgere le altre componenti sanitarie (altri specialisti, infermieri, ecc.), i livelli dirigenziali e soprattutto dei pazienti. Questi i fini delle iniziative toscane illustrate che si propongono di diffondere tali principi nelle attività quotidiane per garantire una sanità saggia e sostenibile.
\end{abstract}

\section{Introduzione}

Il medico nella realtà attuale sperimenta giornalmente come non sempre fare di più significhi fare meglio. Ciò è particolarmente vero per l'internista che si trova di fronte pazienti polipatologici, complessi, spesso critici, frequentemente anziani. La diffusione e l'uso di nuovi trattamenti sanitari e di nuove procedure diagnostiche non sempre si accompagna a maggiori benefici per i nostri pazienti. Spesso interessi economici e ragioni di carattere culturale e sociale spingono all'eccessivo consumo di prestazioni sanitarie, dilatando oltre misura le aspettative più di quanto possa essere realmente attuabile e utile nei singoli casi. Le richieste dei pazienti e delle famiglie, indirizzate da informazioni parziali e a volte distorte dai mass media diventano sempre più pressanti, e rappresentano un punto cardine per atteggiamenti difensivi

Corrispondente: Grazia Panigada, UOC Medicina Interna, Ospedale SS Cosma e Damiano Pescia, Azienda USL3, Pistoia, Italia.

E-mail: g.panigada@alice.it

Parole chiave: choosing wisely, conoscenza, appropriatezza, efficacia, sostenibilità, condivisione, divulgazione, comunicazione.

Articolo pubblicato secondo la Creative Commons Attribution NonCommercial 3.0 License (CC BY-NC 3.0).

CC Copyright G. Panigada et al., 2014

Licensee PAGEPress, Italy

QUADERNI - Italian Journal of Medicine 2014; 2:135-141 da parte dei sanitari, sicuramente non adeguatamente sostenuti dalle istituzioni. L'agire secondo i principi di appropriatezza, efficacia e sostenibilità, rappresenta l'impostazione corretta per un management efficace prima di tutto per il paziente e solo conseguentemente per la struttura. Queste modalità gestionali richiedono un'alleanza medico-paziente fattiva e ben lontano da considerarsi acquisita, ma da conquistarsi mettendo in atto tutte le strategie di comunicazione non solo innate e dettate dall'esperienza, ma anche acquisite e acquisibili attraverso strumenti formativi e di implementazione. Non ultimo è necessario il sostegno delle organizzazioni da conquistare con progetti condivisi e non calati dall'alto col solo fine del contenimento della spesa. Resta comunque che tutte le scelte saggie, non intraprese in maniera routinaria ma basandosi su concreta appropriatezza possano contribuire alla riduzione di sprechi e spese inutili, prevenendo il ricorso a tagli lineari nei confronti di spese realmente utili ed efficaci ai quali talvolta già ora siamo costretti ad assistere.

Dunque ben venga l'adesione della Medicina Interna e con essa della FADOI a iniziative quali il choosing wisely, ${ }^{1}$ campagna promossa dall'American Board of Internal Medicine Foundation con l'obiettivo di incoraggiare i medici, i pazienti e le organizzazioni che si occupano di salute a ragionare criticamente sui test e le procedure mediche che possono non essere necessari e che in qualche caso possono risultare dannosi. In questo contesto appaio sicuramente condivisibili le scelte effettuale da altre società scientifiche in ambito internazionale su liste di esami strumentali, pratiche cliniche, terapie da non eseguire o almeno da valutare criticamente..$^{2-4} \mathrm{Ed}$ 
anche altrettanto positivo è l'aderenza ai programmi di slow medicine $e^{5}$ per una medicina sobria, rispettosa e giusta, che non si fermi ad un elenco di pratiche da non fare ma valuti l'appropriatezza dell'allocazione delle risorse strutturali, organizzative, tecnologiche ma soprattutto umane.

Come società scientifica dobbiamo proporre un vero e proprio cambiamento culturale attraverso formazione e ricerca clinica per impostare un approccio metodologico di governo clinico che insegua le priorità e la personalizzazione delle cure, non in base alla sola patologia ma alle caratteristiche del paziente e consenta all'organizzazione un approccio sostenibile. ${ }^{6}$

\section{Lo scenario internistico}

L'aumento della sopravvivenza e la creazione di fasce di popolazione fragili ha determinato in questi anni un'accresciuta richiesta di salute che si è riversata su tutti i settori della Sanità ma che ha trovato la sua valvola di sfogo privilegiata negli Ospedali. Per le caratteristiche dei pazienti, nonché per il tipo di formazione e attitudine dei medici, la maggior parte del carico assistenziale è stato assorbito dalla Medicina Interna. I pazienti internistici sono per lo più, ma non esclusivamente anziani, hanno molteplici comorbilità croniche, sulle quali si sommano situazioni acute che compromettono l'equilibrio spesso precario. Nessun indice ne definisce correttamente la complessità e gli indicatori imposti dai controlli di gestione non rappresentano adeguatamene pesi e carichi di lavoro necessari a una presa in carico efficace ed efficiente. ${ }^{7}$

Negli ultimi anni la medicina interna che si è fatta carico anche di numerose trasformazioni per adeguarsi al cambiamento. Al suo interno è stato necessario sviluppare una discreta versatilità sia per quanto riguarda la forma di offerta erogata sia per quanto riguarda le conoscenze, dispiegando un variegato ventaglio di competenze che vanno dall'assistenza al paziente $\mathrm{cri}$ tico (ventilazione non invasiva, complicanze settiche, gestione di cateteri venosi centrali, ecc.) al reinserimento sul territorio del paziente vulnerabile, dalla Medicina di Iniziativa ai percorsi assistenziali complessi in regime ambulatoriale. Anche molte tipologie di pazienti che in passato venivano seguiti da altre specialistiche, ora si ritrovano nei setting internistici. I pazienti pre- e post-chirurgici, i politraumatizzati ne sono esempi rilevanti e sempre più diffusi.

In Toscana, per esempio nell'ultimo decennio si è assistito a un incremento di ricoveri per emorragie cerebrali traumatiche in Medicina Interna del 47\%, particolarmente nella fascia di età più avanzata $>75$ anni, ${ }^{8}$ cosa che ha reso necessaria l'acquisizione di competenze specifiche e la condivisione dei casi con specialiste anche lontane come estrazione e come collocazione quali la neurochirurgia, determinando la necessità anche di messa a punto di percorsi di tele medicina. Dunque la Medicina Interna colloca la sua mission tra criticità e cronicità in un'ottica di richieste sempre più pressanti ed estensive, ma con risorse limitate.

Un altro problema di rilievo è rappresentato dal revolving door che interessa soprattutto particolari fasce di patologie croniche quali lo scompenso cardiaco e la broncopneumopatia cronica ostruttiva soprattutto laddove non si siano ottimizzati i rapporti ospedale territorio e i flussi dei pazienti non adeguatamente governati.

Lo studio SMIT (Scompenso Cardiaco nelle Medicine Interne della Toscana ${ }^{9}$ ) mostra che i pazienti ricoverati per scompenso cardiaco nel $22 \%$ dei casi erano già stati ricoverati nei precedenti trenta giorni. Questo dipende certo dalla tipologia di paziente, ma anche dal fatto che il territorio non è ancora pronto al cambiamento.

Da tutto questo consegue l'elevato numero dei ricoveri internistici, l'alto tasso di occupazione dei posti letto e tutti i rischi connessi al sovraffollamento.

Pertanto una gestione snella che consenta nel rispetto dell'evidenza una presa in carico secondo piani diagnostico terapeutici assistenziali ottimizzati per il paziente e non per la malattia non può esimersi da richiedere scelte saggie che definiscano non soltanto cosa fare, ma soprattutto cosa non fare nell'interesse stesso del paziente e congiuntamente di tutto il management sanitario.

\section{Le iniziative della FADOI Toscana}

La FADOI Toscana nell'ultimo anno ha promulgato 3 iniziative per diffondere la cultura della wise medicine.

\section{Adesione al progetto regionale indetto da gestione rischio clinico}

Sollecitata dal gruppo regionale del rischio clinico ha valutato la trasferibilità al contesto toscano delle indicazioni fornite dalle società Americane per individuare le azioni di contenimento più adeguate delle prestazioni non basate su evidenze o addirittura inappropriate. In quest'ottica abbiamo preso parte a iniziative di divulgazione, informazione e formazione fatte dall'organo regionale, ma senza ottenere risultati in termini di miglioramento della metodologia clinica, di dati di monitoraggio, di analisi di flussi e attività di auditing secondo quelle che ci erano state presentate come le finalità del progetto.

\section{Questionario FADOI Toscana}

Più vantaggioso, col fine della diffusione del dibattito, e quindi dello sviluppo di una coscienza condivisa, la somministrazione di un questionario ${ }^{10}$ durante l'ultimo congresso regionale FADOI (Figura 1) per sotto- 
porre al giudizio degli internisti toscani alcuni dei test e procedure indicati come inutili dalle Società Scientifiche Americane e per sollecitare eventuali nuove proposte. Il campione è risultato rappresentativo (Tabella 1). Al di là della condivisione totale o parziale dei singoli ambiti alcune considerazioni devono essere tratte.

Sembra sia presente un'attenzione diffusa e un elevato grado di interesse nei confronti dell'eliminazione di alcune procedure routinarie ma scarsamente utili, tuttavia viene riconosciuta la necessità di coinvolgimento delle altre componenti sanitarie (altri specialisti, infermieri, ecc.), dei livelli dirigenziali, dei pazienti e delle varie componenti della società interessate.

Una domanda diffusa: siamo noi internisti in grado di mettere in atto le enunciazioni sulle quali esiste un accordo unanime o quasi?

In Figura 2 alcuni dei commenti più significativi e in Figura 3 le proposte nel dettaglio che denotano ancora una volta come l'attenzione dell'internista sia rivolta non solo a questioni cliniche ma anche etiche dove forse non si sente né adeguatamente preparato né sufficientemente supportato sul piano istituzionale.

Nel dettaglio gli items discussi hanno ricevuto le seguenti valutazioni.

- Largamente condiviso il richiamo all'inutilità della profilassi dell'ulcera da stress tanto praticata non solo a livello internistico. È ben noto infatti come gli inibitori di pompa, oltre a non essere necessari in molti dei contesti nei quali attualmente vengono indiscriminatamente prescritti, anche in ambito ospedaliero, possono addirittura risultare dannosi . Significativa fra gli altri effetti collaterali l'aumentata suscettibilità verso polmoniti nosocomiali $i^{11,12} \mathrm{e}$ infezioni da clostridio difficile, ${ }^{13}$ nonché problematiche di malassorbimento.

Tabella 1. Campione Questionario FADOI Toscana (25/10/2013).

\begin{tabular}{lc}
\hline Campione & No. \\
\hline Numero & 79 \\
\hline Sesso & $47 \mathrm{M}, 32 \mathrm{~F}$ \\
\hline Età & $51,7 \pm 8,9$ anni \\
\hline Dirigente medico & 56 \\
\hline Direttore struttura complessa & 18 \\
\hline Specializzandi & 2 \\
\hline Altro & 3 \\
\hline
\end{tabular}

Esprimi sulle singole pratiche: condivisione, parziale condivisione, non condivisione

1) Non prescrivere farmaci per la profilassi dell'ulcera da stress a meno che non vi sia un alto rischio di complicazioni gastrointestinali. (Society of Hospital Medicine - Adult Hospital Medicine)

2) Non posizionare o lasciare in sede un catetere vescicale per incontinenza, per comodità di assistenza o per monitorare la diuresi nei pazienti non critici (motivi accettabili di posizionamento del catetere vescicale: paziente critico, ostruzione, hospice, per $<2$ giorni nel periodo perioperatorio, procedure urologiche). Nelle altre condizioni controllare il peso corporeo invece di monitorare la diuresi. (Society of Hospital Medicine - Adult Hospital Medicine)

3) Evitare le trasfusioni di globuli rossi per soglie arbitrarie di emoglobina in assenza di sintomi di sindrome coronarica, insufficienza cardiaca o ictus. (Society of Hospital Medicine - Adult Hospital Medicine)

4) Non ripetere frequentemente gli esami ematici (emocromo e chimica clinica) in presenza di stabilità clinica e di laboratorio. (Society of Hospital Medicine-Adult Hospital Medicine)

5) Non posizionare o mantenere in sede cateteri centrali inseriti per via periferica (PICC) per comodità del paziente o di assistenza. (Society of General Internal Medicine)

6) Non inserire un sondino percutaneo per nutrizione (PEG) nei soggetti con demenza avanzata. Fornisci, invece, assistenza per l'alimentazione orale. (Dedicated to Long Term Care Medicine and American Geriatrics Society)

7) Nei pazienti con bassa probabilità pre-test di tromboembolismo venoso, richiedi il dosaggio del D-dimero ad alta sensibilità come test diagnostico iniziale; non richiedere test di imaging come test diagnostico iniziale. (American College of Physicians)

8) Evitare la radiografia del torace di routine durante il ricovero o preoperatoria nei pazienti senza anamnesi o esame obiettivo significativi. (American College of Radiology)

9) Non eseguire le sub-sierologie degli ANA senza una positività degli ANA ed il sospetto clinico di una malattia immuno-mediata. (American College of Rheumatology)

10) Evita di impiegare l'eco-stress in pazienti asintomatici con basso rischio di malattia coronarica.

(American Society of Echocardiography)

Commenti

Proposte...

(n)

......

Figura 1. Questionario somministrato ai partecipanti al Congresso Regionale FADOI Toscana (25/10/2013). 
- Da condividere col personale infermieristico la buona norma di non posizionare o lasciare in sede un catetere vescicale per incontinenza, per comodità di assistenza o per monitorare la diuresi nei pazienti non critici. Risultano altresì motivi accettabili di posizionamento situazioni critiche, con ostruzione, in hospice, oppure per $<2$ giorni nel periodo perioperatorio, e per procedure urologiche. Il controllo del peso corporeo potrà sostituire il monitoraggio della diuresi laddove risulti impraticabile. È ampiamente dimostrato come l'uso del catetere vescicale incrementi il rischio infettivo ${ }^{14}$ anche attuando protocolli gestionali adeguati, con conseguentemente aumento di morbiltà e mortalità e spese sanitarie evitabili. - Approvato dal più del $70 \%$ degli internisti toscani il richiamo a evitare le trasfusioni di globuli rossi per soglie arbitrarie di emoglobina in assenza di sintomi di sindrome coronarica, insufficienza cardiaca o ictus. Largamente diffuse nelle numerose realtà internistiche rappresentate strategie trasfusionali restrittive che richiedano una valutazione completa delle condizioni del paziente e non basano la procedura sul singolo valore di emoglobina. ${ }^{15}$

- Accettata senza apparente riserva l'indicazione a non ripetere frequentemente gli esami ematici se non giustificati da variazioni cliniche durante la de-

$\checkmark$ Molti degli items vanno condivisi con il personale infermieristico

$\checkmark$ Profilassi ulcera: non ci sono studi nei pazienti molto anziani...

$\checkmark$ Catetere vescicale: condividere con il personale infermieristico

$\checkmark$ Trasfusioni: indicazioni dei centri trasfusionali diverse da un ospedale all'altro anche nella stessa regione... Necessità di condivisione con altre specialistiche

$\checkmark$ PEG: legislazione non chiara e difficile rapporto con $i$ familiari

$\checkmark$ PEG: salvo disfagia assoluta

$\checkmark$ PEG: lasciare uno spazio al SNG in casi selezionati...

$\checkmark$ PEG: campagne di sensibilizzazione della popolazione

$\checkmark$ PEG: credo che una PEG in un demente disfagico sia la possibilità migliore...

$\checkmark$ PEG: ...la malnutrizione è una malattia che va prevenuta e trattata...

$\checkmark$ RX torace: ma di quel $2 \%$ in cui può essere utile e non viene effettuato, cosa accade?

$\checkmark$ se non sospetto una malattia autoimmune non eseguo ANA... Se ho il sospetto clinico eseguo ANA e subset (anti ENA, anti dsDNA etc) che ritengo appropriati per lo specifico fin dall'inizio.

$\checkmark$ tutti condivisibili in teoria, non sempre praticabili

Figura 2. Commenti questionario FADOI (25/10/2013).

sospendere la terapia antibiotica e la NPT/NET nel fine vita

....altre procedure inutili

$\checkmark T C$ cranio senza mdc in caso di lipotimia con clinica muta per deficit focali;

$\checkmark$ Ecocolor doppler arterioso di screening arteriopatia con ABI normale

$\checkmark$ non eseguire urincoltura in assenza di sintomi

$\checkmark$ non ripetere gli esami ematici eseguiti in PS il giorno prima senza una indicazione clinica

$\checkmark \quad$ non eseguire markers tumorali ad ampio raggio senza un sospetto clinico preciso...

Figura 3. Proposte Questionario FADOI (25/10/2013). 
genza. Ma nonostante l'inutilità se non il danno ${ }^{16}$ dettato da questa pratica, sicuramente è necessario un richiamo costante all'applicazione di questa norma. Così come è necessario ribadire la necessità valutare l'appropriatezza di qualsiasi indagine prima della prescrizione. Utile porsi le classiche 3 domande: sarà davvero utile? è già stato fatto? potrà essere dannoso ${ }^{17}$

- Apprezzato anche l'appello a non posizionare o mantenere in sede cateteri centrali anche se inseriti per via periferica per comodità del paziente o di assistenza. Questa procedura, largamente diffusa nei setting internistici anche in virtù della sua implementazione con l'utilizzo dell'eco bed side, in cui tanto come organizzazioni (FADOI/ANIMO) ci siamo prodigati, non è scevra da possibili complicanze infettive e tromboemboliche ${ }^{18}$ che devono essere attentamente ponderate prima di darne indicazione coinvolgendo nella decisione paziente, famiglia e anche il personale infermieristico.

- Molto sentita la discussione sulla norma relativa all'inserimento della PEG nei paziente con demenza avanzata, laddove l'approccio geriatrico consiglia invece adeguata assistenza nell'alimentazione orale ricordando come la metodologia transcutanea può incrementare il rischio di agitazione, favorire le infezioni senza risolvere la problematica dell'ab ingestis $^{19}$ causa non rara di ospedalizzazione. I commenti richiamano l'importanza di un'adeguata nutrizione, la possibilità di utilizzo del sondino naso gastrico come metodologia più transitoria, la necessità di un'alleanza con la famiglia del paziente mediata attraverso una corretta comunicazione non così scontata. Viene anche richiamata la necessità di normative legislative appropriate.

- Condiviso lo step iniziale e conclusivo del dosaggio del D-Dimero nella diagnostica del tromboembolismo venoso nel paziente con bassa probabilità pretest anche se la pratica corrente nelle nostre realtà vede ormai affermato l'uso della diagnostica ultrasonorografica, anche laddove sicuramente eccessiva. In pazienti con bassa probabilità pre-test di tromboembolismo venoso (TEV) come definito dalle regole predittive di Wells infatti, una misurazione negativa del D-Dimero ad alta sensibilità esclude efficacemente il TEV e la necessità di ulteriori studi di imaging. ${ }^{20}$

- Un 10\% degli internisti intervistati non accetta di evitare la radiografia del torace di routine durante il ricovero nei pazienti senza anamnesi o esame obiettivo significativi, e più del $20 \%$ esprime perplessità e critiche, anche se è dimostrato che soltanto il $2 \%$ di queste immagini porta a un cambiamento nella gestione. ${ }^{21}$ Nella realtà internistica, peraltro, è comunque elevato il numero dei pazienti con sintomatologia respiratoria e obiettività toracica patologica che ne giustificano l'esecuzione semmai si potrebbe riflettere su quando sostituirla con ecografia bed side.

- Largamente accettato la non utilità di eseguire le sub-sierologie degli ANA senza una positività degli ANA ed il sospetto clinico di una malattia immunomediata. Gli anticorpi anti-DNA nativa e gli ENA sono di solito negativi se l'ANA è negativo con limitate eccezioni. Ancora una volta la scelta saggia sarà guidata dal sospetto clinico di una specifica malattia e non da una lista preconfezionata che se da un lato facilita la routine è sicuramente induttrice di spesa ingiustificata e rischia di porre il paziente di fronte a una risposta patologica non comprovata dal contesto rendendo così necessari controlli seriati ancor più inutili e inappropriati. ${ }^{22}$

- Del tutto condiviso l'inutilità del ricorso all'ecostress in pazienti asintomatici con basso rischio di malattia coronarica. Approvata in ambito cardiologico la norma che esami di stress cardiaco o avanzati test di imaging anche se non-invasivi, in pazienti senza sintomi o in un pattern seriale o programmato, esitano raramente in qualche significativo cambiamento della gestione ma nel contempo possono condurre a procedure invasive non necessarie e ad eccesso di esposizione a radiazioni senza nessun impatto provato sugli esiti del paziente. ${ }^{23}$

\section{Workshop di Medicina vascolare: scelte sagge nel management del paziente con malattia cerebrovascolare}

Terza iniziativa di largo impatto a livello internistico in Toscana l'ormai consueto appuntamento annuale di medicina vascolare improntato quest'anno alla divulgazione di scelte saggie nella gestione della patologia cerebrovascolare di grande interesse in ambito internistico.

Il 70\% degli stroke, infatti nella nostra regione vengono ricoverati in Medicina Interna. La diagnostica vascolare è affidata in larga percentuale agli internisti. Le urgenze vascolari differibile e tra queste l'ischemia cerebrale transitoria vengono gestite in day service dagli internisti con percorsi condivisi con i dipartimenti di emergenza.

Scelta saggia documentata e condivisa è il ricovero dei pazienti con stroke in aree di degenza ad alta intensità, realizzate in molte realtà toscane nei setting di area medica, in virtù della diffusione dei principi dell'intensità di cure. In tali contesti dovranno essere erogate quelle modalità di monitoraggio, diagnostica, terapia e riabilitazione precoce multiprofessionali analoghe a quelle ottenibili nelle più classiche stroke unit sicuramente più dispendiose e meno eque.

Altrettanto saggio improntare una diagnostica efficace alla definizione della patogenesi per una corretta profilassi secondaria.

Molto saggio diffondere l'utilizzo della fibrinolisi 
sistemica in tutti i nostri ospedali in collaborazione con la medicina d'urgenza.

Sicuramente ogni scelta effettuata nel rispetto di indicazioni e di appropriatezza avrà un impatto non solo clinico ma anche gestionale.

Nel workshop è stata ribadita e comprovata da evidenze la necessità di applicare appropriatezza nella diagnostica ecocolordoppler che assorbe risorse internistiche meglio sfruttabili nella gestione dei pazienti in percorsi definiti.

Limitando l'esame al paziente sintomatico o all'asintomatico solo nei casi indicati dalle linee guida e raccomandando una tempistica di controlli efficace ${ }^{24,25}$ potremmo sicuramente ridurre il numero di esami eseguiti senza nulla togliere ai pazienti.

Nella mia Unità Operativa nei primi tre mesi del 2014, dei pazienti valutati soltanto un $30 \%$ ha ottenuto un valore aggiunto dall'ecocolordoppler, alta è stata la percentuale dei casi risultati fuori indicazione, che comunque hanno assorbito risorse che potevano essere meglio utilizzate (Tabella 2).

Applicando scelte sagge, dal governo della domanda alla concessione di accessi facilitati nei casi condivisi attraverso percorsi ospedale territorio definiti anche la diagnostica vascolare potrebbe essere governabile. Necessario conoscere, nelle singole realtà l'entità del fenomeno, condividere la problematica mediante scambi costanti tra specialistiche e medicina generale, definire linee guida aziendali, assegnare obiettivi realizzabili con le risorse disponibili.

\section{Conclusioni}

In un'epoca in cui l'appropriatezza viene quotidianamente rivendicata non solo come politica economica, ma anche come garanzia di equità, di corretta allocazione delle (poche) risorse e di giustizia sociale, cosa significa quindi per l'internista scegliere saggiamente?

Può voler dire perseguire la diagnosi microbiologica per poter usare un antibiotico poco costoso e ugualmente efficace in modo da evitare l'insorgenza di pericolose e costose resistenze.

Può essere saggio astenersi da manovre invasive

Tabella 2. Medicina Interna - Ospedale SS. Cosma e Damiano, Pescia (PT) - Azienda USL 3 Pistoia.

Numero pazienti inviati per eseguire ecocolordoppler (primi 3 mesi 2014)

\begin{tabular}{lc}
\hline Totale & 350 \\
\hline Pazienti con indicazione & 105 \\
\hline Pazienti fuori indicazioni & 245 \\
\hline Day service attivati per urgenze vascolari differibili & 28 \\
\hline
\end{tabular}

nel fine vita o da dannose nutrizioni artificiali in pazienti terminali, dedicandosi piuttosto alla comunicazione con i familiari o all'educazione sanitaria (e filosofica) di una società in cui domina sempre più la mancanza di cultura e l'idea della perfezione e dell'immortalità.

Può significare dedicare più tempo all'anamnesi per evitare inutili e a volte sbagliati esami diagnostici.

Può significare scegliere un regime ambulatoriale anziché di ricovero per gestire anche patologie complesse quando ciò non comporti rischi o disagi eccessivi per l'ammalato, nonché circostanziare la durata della degenza alle reali necessità.

Può essere la riscoperta del legame relazionale medico-paziente ormai violentato e svilito dalla (dis)informazione dei media, dagli spauracchi medicolegali e dai ritmi di lavoro frenetici a cui l'internista è, forse più di altri specialisti costretto.

Si potrebbe andare avanti all'infinito ad elencare situazioni della vita di tutti i giorni in cui siamo chiamati, nonostante tutte le insidie, le difficoltà e la stanchezza, ad esercitare saggiamente il nostro ruolo di medici.

Se ci pensiamo attentamente, riconosceremo che ciascuna di queste occasioni è riuscita quando è sottesa dalla conoscenza scientifica, base imprescindibile di ogni scelta saggia, dalla curiosità, che sola ci può permettere di continuare a studiare e a cercare la soluzione dei problemi, dall'onestà intellettuale, che ci deve sempre portare al confronto e all'autocritica, dalla tenacia, che ci guida attraverso gli ostacoli fino all'obiettivo e non ultimo dalla tensione morale, ricordando, come ha detto qualcuno, che il futuro dipende da quello che facciamo nel presente.

\section{Bibliografia}

1. ABIM Foundation. Choosing wisely: an initiative of the ABIM Foundation; 2013. Available from: http://www. choosingwisely.org

2. Morden EM, Colla CH, Sequist TD, Rosenthal MB. Choosing wisely - the politics and economics of labeling low-value services. N Engl J Med 2014;370:589-59.

3. Volpp KG, Loe WG, Asch DA. Choosing wisely: low value services, utilization, and patient cost sharing. JAMA 2012;308:1635.

4. Cassel CK, Guest JA. Choosing wisely: helping physicians and patients make smart decisions about their care. JAMA 2012;307:1801-2.

5. Slow Medicine. Fare di più non significa fare meglio; 2012. Disponibile a: http://slowmedicine.it

6. Nardi R, Berti F, Fabbri LM, et al. Toward a sustainable and wise healthcare approach: potential contributions from hospital Internal Medicine Departments to reducing inappropriate medical spending. Ital $\mathrm{J}$ Med 2013;7:65-81.

7. Nardi R, Scanelli G, Corrao S, et al. Co-morbidity does not reflect complexity in internal medicine patients. Eur J Intern Med 2007;18:359-68. 
8. Masotti L, Lorenzini G, Chiti I, et al. Traumatic brain injuries in Internal Medicine wards: an emerging challenge needing for a call to action. Ital J Med 2014 [submitted].

9. Verdiani V, Panigada G, Montagnani A, Masotti L. Lo scompenso cardiaco nella Medicina interna della Toscana. Ital J Med 2014;8(s2):133-34.

10. Fortini A, Tavernese G, Panigada G, Tafi A. Choosing wisely: questionnaire FADOI Tuscany. Ital J Med 2014;8(s2): 51.

11. Fohl AL, Regal RE. Proton pump inhibitor-associated pneumonia: not a breath of fresh air after all? World J Gastrointest Pharmacol Ther 2011;2:17-26.

12. Herzig SJ, Howell MD, Ngo LH, Marcantonio ER. Acid-suppressive medication use and the risk for hospital-acquired pneumonia. JAMA 2009;301:2120-8.

13. Aseeri M, Schroeder T, Kramer J, Zackula R. Gastric acidsuppression by proton pump inhibitors as a risk factor forclostridium difficile-associated diarrhea in hospitalized patients. Am J Gastroenterol 2008;103:2308-13.

14. Hooton TM, Bradley SF, Cardena DD, et al. Diagnosis, prevention, and treatment of catheter-associated urinary tract infection in adults: 2009 International Clinical Practice Guidelines from the Infectious Diseases Society of America. Clin Infect Dis 2010;50:625-63.

15. Carson JL, Grossman BJ, Kleinman S, et al. Red blood cell transfusion: A clinical practice guideline from the AABB. Ann Intern Med 2012;157:49-58.

16. Salisbury AC, Reid KJ, Alexander KP, et al. Diagnostic blood loss from phlebotomy and hospital-acquired anemia during acute myocardial infarction. Arch Intern Med 2011;171:1646-53.

17. Schattner A. Test appropriateness index. Am J Med 2012;125:e13.

18. Chopra V, Anand S, Krein SL, et al. Bloodstream infection, venous thrombosis, and peripherally inserted central catheters: reappraising the evidence. Am J Med 2012;125:733-74.
19. Hanson LC, Carey TS, Caprio AJ, et al. Improving decision-making for feeding options in advanced dementia: a randomized, controlled trial. J Am Geriatr Soc 2011;59:2009-16.

20. Bates SM, Jaeschke R, Stevens SM, et al. Diagnosis of DVT antithrombotic therapy and prevention of thrombosis, 9th ed. American College of Chest Physicians Evidence-Based Clinical Practice Guidelines. Chest 2012;141:e351S-418S.

21. Gupta SD, Gibbins FJ, Sen I. Routine chest radiography in the elderly. Age Ageing 1985;14:11-4.

22. Tozzoli R, Bizzaro N, Tonutti E, et al. Guidelines for the laboratory use of autoantibody tests in the diagnosis and monitoring of autoimmune rheumatic diseases. Am J ClinPathol 2002;117:316-24.

23. Bobbio $\mathrm{M}$, Abrignani $\mathrm{M}$, Caldarola $\mathrm{P}$, et al. Fare di più non significa fare meglio: le proposte dell'AMNCO. G Ital Cardiol 2014;15:4.

24. Thapar A, Jenkins IH, Mehta A, Davies AH. Diagnosis and management of carotid atherosclerosis BMJ 2013;346:f1485.

25. Mohler ER III, Gornik HL, Gerhard-Herman M, et al. ACCF/ACR/AIUM/ASE/ASN/ICAVL/SCAI/SCCT/SI R/SVM/SVS 2012 Appropriate Use Criteria for Peripheral Vascular Ultrasound and Physiological Testing Part I: Arterial Ultrasound and Physiological Testing: A Report of the American College of Cardiology Foundation Appropriate Use Criteria Task Force, American College of Radiology, American Institute of Ultrasound in Medicine, American Society of Echocardiography, American Society of Nephrology, Intersocietal Commission for the Accreditation of Vascular Laboratories, Society for Cardiovascular Angiography and Interventions, Society of Cardiovascular Computed Tomography, Society for Interventional Radiology, Society for Vascular Medicine, and Society for Vascular Surgery. J Am Coll Cardiol JACC 2012;60:242-76. 


\title{
Il percorso del tromboembolismo venoso nell'ospedale per intensità di cure: paradigma di wise medicine
}

\author{
Luca Masotti, ${ }^{1}$ Irene Chiti, ${ }^{2}$ Giancarlo Landini, ${ }^{1,3}$ Niccolò Napoli, ${ }^{4}$ Grazia Panigada ${ }^{2}$ \\ ${ }^{1}$ UOC Medicina Interna, Ospedale Santa Maria Nuova, AS Firenze, ASF-Firenze; ${ }^{2}$ UOC Medicina Interna, Ospedale SS \\ Cosma e Damiano Pescia, Azienda USL3, Pistoia; ${ }^{3}$ Dipartimento di Medicina e Specialistiche Mediche, AS di Firenze; \\ ${ }^{4}$ Scuola di Specializzazione in Chirurgia Generale Università di Pisa, UOC Chirurgia Generale e Trapianti nell'uremico e nel \\ diabetico, AOU Pisana, AOUP-Pisa, Italia
}

\begin{abstract}
RIASSUNTO
Scegliere saggiamente (choosing wisely) comporta la definizione di percorsi diagnostico terapeutici basati sull'evidenza scientifica e circostanziati al paziente e alla realtà assistenziale. L'organizzazione per intensità di cure può facilitare la modulazione del flusso dei pazienti con tromboembolismo venoso, dall'alto rischio nel livello intensivo/sub-intensivo al rischio intermedio nel livello 2 fino al a basso rischio gestibile ambulatorialmente attraverso i day service che ne garantiscono una presa in carico efficace e sicura. Ormai largamente diffusa la gestione domiciliare della trombosi venosa profonda, potrà essere scelta saggia anche dimettere rapidamente dopo breve osservazione o addirittura non ricoverare anche i pazienti con embolia polmonare a basso rischio dopo una attenta stratificazione del rischio di mortalità, di recidiva tromboembolica e di sanguinamento garantendo comunque un adeguato follow-up.
\end{abstract}

\section{Introduzione}

Il tromboembolismo venoso (TEV), che comprende trombosi venosa profonda (TVP) ed embolia polmonare $(E P)$ è una delle principali e più diffuse patologie del sistema cardio-circolatorio. Nei Paesi occidentali si calcola sia la terza emergenza cardiovascolare dopo la cardiopatia ischemica e l'ictus, con una incidenza, etàdipendente, di 1/1000 abitanti/anno. ${ }^{1}$ Malattia grave e potenzialmente fatale è soggetta a recidive, gravata da possibili complicanze a distanza quali la sindrome posttrombotica e l'ipertensione polmonare. ${ }^{1}$

Il management del TEV può rappresentare uno dei prototipi di percorsi diagnostico-terapeutici basati su scelte sagge. In effetti già alcune Società Scientifiche hanno inserito, tra le loro 5 raccomandazioni choosing wisely, indicazioni riferite al TEV. ${ }^{2}$

Corrispondente: Grazia Panigada, UOC Medicina Interna, Ospedale SS Cosma e Damiano Pescia, Azienda USL3, Pistoia, Italia.

E-mail: g.panigada@alice.it

Parole chiave: ospedale per intensità di cure, percorsi ospedale-territorio, choosing wisely, tromboembolismo venoso, diagnosi, trattamento domiciliare.

Articolo pubblicato secondo la Creative Commons Attribution NonCommercial 3.0 License (CC BY-NC 3.0).

CCopyright L. Masotti et al., 2014

Licensee PAGEPress, Italy

QUADERNI - Italian Journal of Medicine 2014; 2:142-147
L'effettuazione della diagnosi di TEV non può prescindere dal sospetto clinico. Quest'ultimo nasce da anamnesi, esame obiettivo e nell'EP, da esami strumentali di primo livello (radiografia al torace, elettrocardiogramma, emogasanalisi). La conferma diagnostica di TEV è affidata ad esami strumentali non invasivi e largamente diffusi come l'ultrasonografia, ma anche a procedure non sempre scevre da possibili effetti collaterali quali angio-tomografia computerizzata (TC) polmonare che richiede l'utilizzo di mezzo di contrasto iodato (MDC), potenzialmente gravato da reazioni allergiche o da danni iatrogeni quali la nefropatia da MDC. ${ }^{3}$ Inoltre angio-TC polmonare e scintigrafia polmonare, espongono il paziente ad alte dosi di radiazioni ionizzanti con incremento del rischio di neoplasie solide e/o ematologiche o, nel caso di donne in gravidanza, al rischio teratogeno. Da non sottovalutare il costo, non trascurabile, di queste procedure diagnostiche. Risulta pertanto fondamentale, prima di esporre un paziente a tali rischi e per non sovraccaricare il sistema sanitario di indagini non appropriate, la stima della probabilità clinica pre-test. ${ }^{4}$ Ciò è possibile mediante l'utilizzo di score, quali quelli di Wells per TVP o EP o quello di Ginevra per EP, associati al dosaggio del D-Dimero. Questi score suddividono i pazienti in alta o non alta probabilità clinica pre-test.

Nel work-up diagnostico del paziente con sospetto TEV, l'associazione negatività del D-Dimero ad alta sensibilità e non alta probabilità clinica pre-test esclude la diagnosi. ${ }^{3,4}$ In questo gruppo di pazienti scegliere saggiamente significa non proseguire con l'esecuzione di esami strumentali di conferma, cercando diagnosi alternative al TEV. ${ }^{2}$ Viceversa scegliere sag- 
giamente nel work-up diagnostico del TEV nel paziente ad alta probabilità clinica pre-test significa non sottoporre il paziente a dosaggio del D-Dimero e proseguire con indagini strumentali di conferma. ${ }^{2-4}$ In questo caso infatti, un D-Dimero negativo non dovrebbe interrompere l'iter diagnostico e pertanto il suo dosaggio non sarebbe appropriato e comporterebbe una spesa inutile.

\section{Choosing wisely nel tromboembolismo venoso}

Scegliere saggiamente nell'iter diagnostico della TVP significa sottoporre il paziente con probabilità non bassa a echocolor doppler completo, evitando la necessità di ripetere l'esame a 7 giorni se viene esaminato con ultrasonografia a compressione (CUS) solo il distretto prossimale, tanto più che la strumentazione attualmente disponibile consente di eseguire facilmente la CCUS (complete compression ultrasound dopo un training adeguato). ${ }^{5}$
Scegliere saggiamente nell'iter diagnostico della dell'EP significa eseguire una CUS degli arti inferiori dopo la stima della probabilità clinica pre-test associata al D-Dimero e prima dell'esecuzione di eventuali esami di conferma diagnostica (Figura 1). Nel paziente che ha alta probabilità clinica pre-test $\mathrm{o}$ in quello che ha bassa probabilità clinica pre-test ma DDimero positivo, l'esecuzione di una CUS può interrompere l'iter diagnostico dell'EP, confermandola indirettamente, se evidenziasse una TVP prossimale. ${ }^{3}$ Rappresenta sicuramente un percorso saggio perché a più basso costo ed a più basso rischio di effetti collaterali essendo la CUS un esame non invasivo, ed è particolarmente appropriato in pazienti anziani, nelle giovani donne in gravidanza, nei pazienti con allergia al MDC, nei pazienti con moderata-severa insufficienza renale, nei pazienti con ipertiroidismo od in quelli con mieloma multiplo, condizioni in cui l'esposizione a radiazioni o MDC potrebbe risultare dannosa. Nel paziente in cui il sospetto di EP sia alto, la

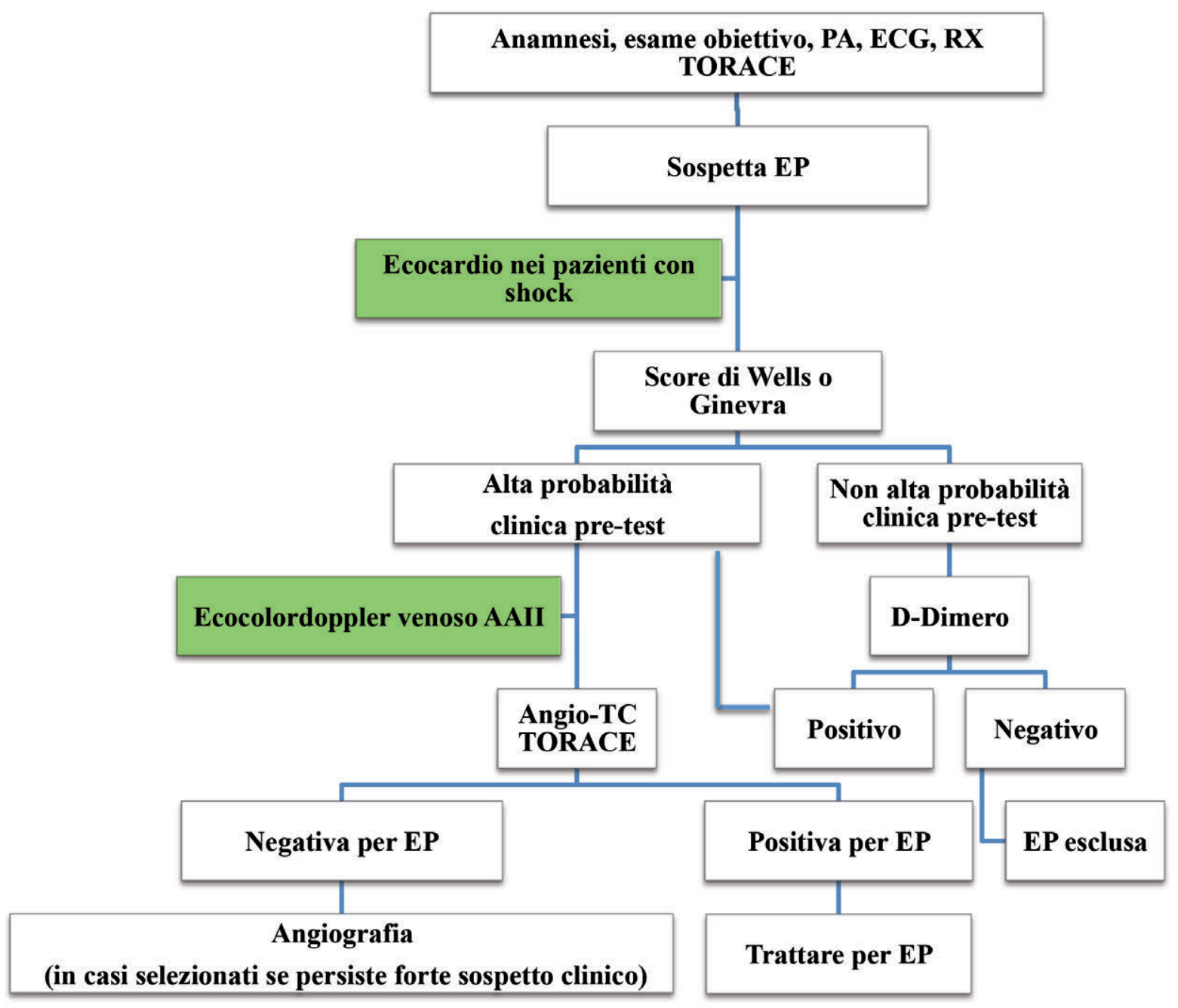

Figura 1. Work-up diagnostico dell'embolia polmonare. 
negatività della CUS non dovrebbe, comunque, interrompere l'iter diagnostico dato che può essere presente una EP isolata o la sorgente dell'embolo potrebbero non essere gli arti inferiori, ma la pelvi, la vena cava inferiore, il cuore o gli arti superiori.

\section{Il tromboembolismo venoso nell'ospedale per intensità di cure}

Scegliere saggiamente nel TEV significa anche personalizzare terapie e setting assistenziali appropriati al rischio di mortalità in fase acuta, recidiva tromboembolica o complicanze emorragiche; e in questo un'organizzazione per intensità delle cure con i differenti livelli può facilitarne la modulazione (Figura 2) ${ }^{6}$

Negli ultimi anni si è largamente affermata la gestione domiciliare della TVP. Tutto questo è stato possibile grazie alla diffusione della diagnostica ultrasonorografica, alla semplificazione delle terapie con l'utilizzo delle eparine a basso peso molecolare e del fondaparinux e, più di recente, degli anticoagulanti orali diretti. Ma, soprattutto, tutto questo è stato possibile grazie alla definizione, nelle singole realtà, di percorsi ospedale-territorio che, con attivazione di interazioni professionali multidisciplinari, hanno permesso di garantire una presa in carico complessiva, dalla diagnosi alla terapia al follow-up dei pazienti affetti da TVP rendendo attuabile, in sicurezza, una gestione solo in apparenza meno complessa del tradizionale ricovero. In questo contesto, rappresenta scelta saggia l'individuazione dei pazienti che devono essere ospedalizzati per condizioni particolarmente complesse per le comorbilità, il rischio emorragico o per la complessità assistenziale. ${ }^{7}$
Nell'EP il management pratico non può ormai più prescindere dalla stima del rischio di mortalità in fase acuta, così come suggerito dalle linee guida dell' $\mathrm{Eu}$ ropean Society of Cardiology (ESC) ${ }^{3}$ Parametri emodinamici (pressione arteriosa), indagini finalizzate alla ricerca di disfunzione cardiaca del cuore destro (ecocardiogramma e/o dosaggio dei peptidi natriuretici) e/o danno miocardico (dosaggio delle troponine) possono permettere di classificare il paziente in alto rischio (paziente in stato di shock, emodinamicamente instabile, mortalità $>15 \%$ ), intermedio rischio (paziente emodinamicamente stabile con presenza di almeno uno dei seguenti reperti: disfunzione cardiaca destra documentata con ecocardio o biomarcatori; danno miocardico evidenziato dal dosaggio della troponina, mortalità 3-15\%) o basso rischio (assenza di disfunzione cardiaca destra o danno miocardico, mortalità $<1 \%$ ) di mortalità in fase acuta. ${ }^{3}$ Scegliere saggiamente nel management pratico dell'EP significa quindi individuare i pazienti ad alto rischio, che necessitano di trattamenti più aggressivi (trombolisi, embolectomia via catetere o chirurgica) e che devono essere assistiti in setting ad alta intensità di cure (livello 1 dell'Ospedale per Intensità di Cure, area intensiva/sub-intensiva) oppure i pazienti ad intermedio rischio che, data la possibilità di deterioramento emodinamico, dovrebbero essere monitorati in setting subintensivi (livello 1B) o di high care medica (livello 2A). Infine di fondamentale importanza l'individuazione dei pazienti a basso rischio di mortalità che, secondo le indicazioni dell'ESC, potrebbero essere dimessi rapidamente dall'Ospedale o direttamente dal DEA o dopo breve ricovero in degenza ordinaria (livello 2 senza monitorizzazione o livello 3) (Figura 3). Il modello prognostico proposto quest'anno dall'ESC
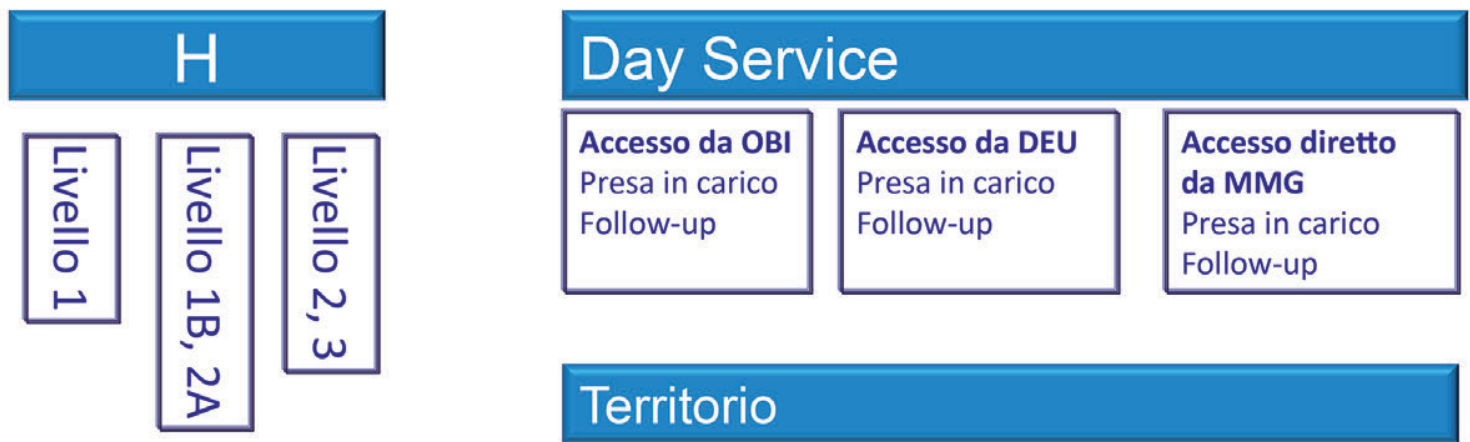

\title{
Territorio
}

\author{
-Stratificazione prognostica (ESC prognostic model, PESI score semplificato) \\ -Rischio di sanguinamento (RIETE score) \\ - Comorbidità \\ -Complessità assistenziale
}

Figura 2. Flusso del paziente con tromboembolismo venoso modulato per intensità di cure. 
risulta particolarmente adatto al paziente con comorbilità ricoverato nei Reparti di Medicina in quanto utilizza anche il PESI score. ${ }^{8}$ Nello studio TUSCAN-PE, recentemente condotto in Toscana e relativo al management dell'EP nei Reparti di Medicina Interna, è stato evidenziato che i pazienti con PESI score semplificato a basso rischio, cioè con assenza di valori di pressione arteriosa sistolica $<100 \mathrm{mmHg}$, frequenza cardiaca $>110 \mathrm{bpm}$, saturazione arteriosa di ossigeno $<90 \%$, anamnesi di neoplasia, scompenso cardiaco o broncopneumopatia cronica ostruttiva, hanno presentato una mortalità dello $0 \%{ }^{9}$

Nonostante i modelli prognostici descritti siano disponibili da alcuni anni, la gestione dei pazienti con EP non sembra differire in base alla stima del rischio di mortalità in fase acuta; ciò è evidenziato dalla dimostrazione, nello studio TUSCAN-PE, che la durata di degenza dei pazienti con EP ad alto, intermedio o basso rischio non differisce, risultando per tutte le classi, in media, non inferiore ai 10 giorni. ${ }^{9}$ Ed allora la sfida per il futuro, nell'ambito del management dell'EP, sarà quella di fare scelte di gestione appropriate e modulate in base al rischio clinico con la tendenza verso la rapida dimissione ed il trattamento domiciliare dell'EP a basso rischio.

I nuovi farmaci anticoagulanti orali potranno facilitare il management dell'EP a basso rischio, date le loro caratteristiche farmacocinetiche (pressoché immediato inizio d'azione) e farmacodinamiche (effetto anticoagulante prevedibile, assenza di necessità di mo- nitorizzazione laboratoristica routinaria) e ciò potrà permettere dimissioni più rapide o addirittura ritorni rapidi al domicilio dopo inquadramento diagnostico ed iniziale trattamento e monitoraggio al DEA in osservazione breve intensiva. ${ }^{10}$ In effetti le note difficoltà di management che si hanno con i farmaci vitamina $\mathrm{K}$ antagonisti, che richiedono un periodo di sovrapposizione con la terapia parenterale dato il lungo periodo di latenza prima di raggiungere il target terapeutico rappresentato da valori di INR compresi tra 2,0 e 3,0, potrebbe rappresentare una delle ragioni che hanno, fino ad oggi, limitato le rapide dimissioni o la gestione domiciliare dell'EP a basso rischio. Nel già citato studio TUSCAN-PE, più dell' $80 \%$ dei pazienti con EP a basso rischio è stato sottoposto a terapia con vitamina $\mathrm{K}$ antagonisti contro meno del $50 \%$ dei pazienti a intermedio od alto rischio. ${ }^{9}$

Il rischio di sanguinamento rappresenta uno dei principali timori che si hanno nella gestione delle patologie che richiedono l'uso di farmaci anticoagulanti. Nell'ambito del TEV è stato proposto uno score, denominato RIETE score dal nome dello studio da cui è stato derivato, che permette di identificare i pazienti che sono a rischio di sanguinamento una volta sottoposti a terapia anticoagulante. ${ }^{11}$ Tra questi fattori sono da annoverare l'età avanzata, l'anemia, la piastrinopenia, la presenza di una coagulopatia, ecc. Diviene quindi fondamentale, nell'ottica della gestione ambulatoriale del TEV identificare il paziente anche a basso rischio di sanguinamento.

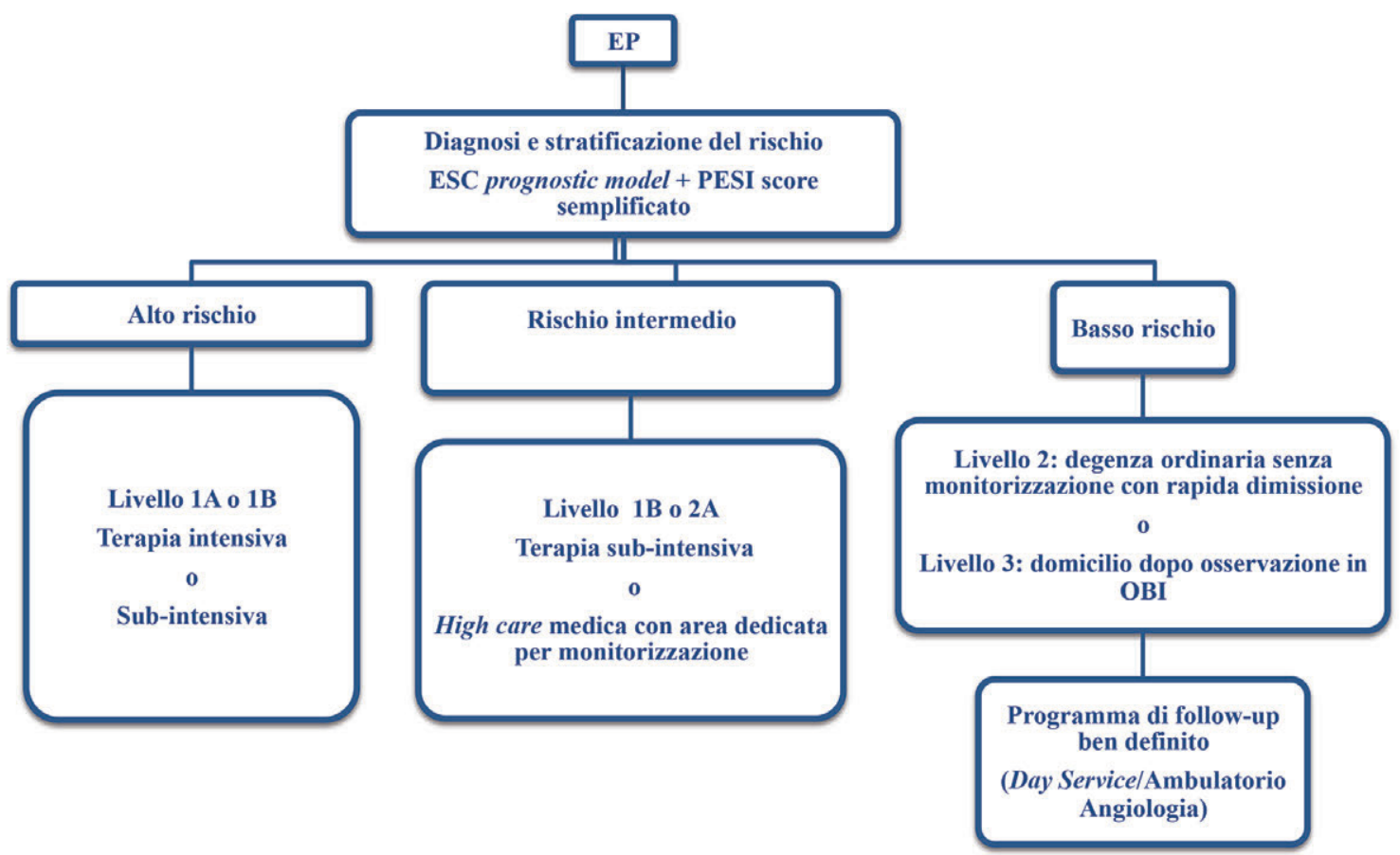

Figura 3. Organizzazione per intensità assistenziale dell'embolia polmonare in fase acuta nell'Ospedale per Intensità di Cura. 


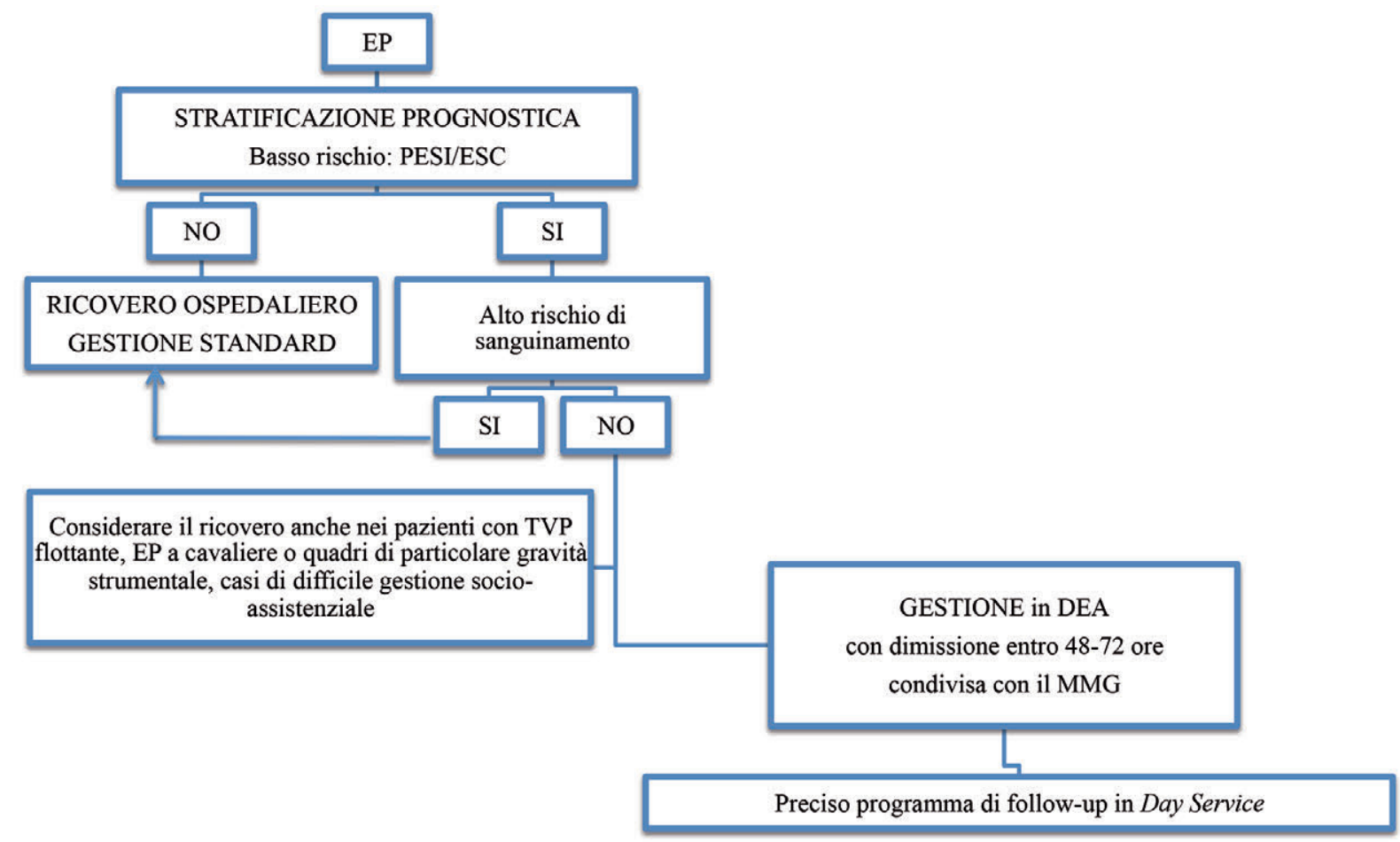

Figura 4. Proposta di flow-chart gestionale dell'embolia polmonare a basso rischio.

\section{Conclusioni}

Se da tempo abbiamo condiviso con la medicina generale e il DEA i percorsi per la gestione domiciliare della TVP garantendo accessi facilitati alla diagnostica vascolare e percorsi di day service per il post-acuto che consentissero la diagnosi patogenetica e il followup preordinato, è forse arrivato il momento di estendere la sfida della gestione domiciliare dell'EP a basso rischio. Ovviamente tutto questo richiede un approccio multidisciplinare che coinvolga territorio ed ospedale con le differenti componenti specialistiche con l'obiettivo di porre al centro il paziente. L'Internista, così come già fatto per la TVP, potrà fare da punto di riferimento tra i Medici del DEA ed i Medici di Medicina Generale, utilizzando quelle stesse interfacce tra Ospedale e Territorio quali il Day Service per affrontare le possibili problematiche inerenti la terapia anticoagulante, gli approfondimenti diagnostici che, inevitabilmente, dovranno essere effettuati nelle forme idiopatiche (screening neoplastico, screening trombofilico, ecc.) ed il follow-up (durata terapia anticoagulante, eventuale prosecuzione o sospensione).

La Figura 4 riassume una proposta di flow-chart gestionale dell'EP a basso rischio nell'ottica di scelte sagge.

\section{Bibliografia}

1. Wong P, Baglin T. Epidemiology, risk factors and sequelae of venous thromboembolism. Phlebology 2012;27:2-11.

2. ABIM Foundation. Choosing wisely: an initiative of the ABIM Foundation; 2013. Available from: http://www. choosingwisely.org

3. Konstantinides S, Torbicki A, Agnelli G, et al. 2014 ESC Guidelines on the diagnosis and management of acute pulmonary embolism. Eur Heart J 2014;35:3033-73.

4. Bounameaux H, Perrier A, Righini M. Diagnosis of venous thromboembolism: an update. Vascular Med 2010;15:399-406.

5. Schellong S, Schwarz T, Halbritter H, et al. Complete compression ultrasonography of the leg veins as asingle test for the diagnosis of deep vein thrombosis. A prospective clinical outcome study. Thromb Haemost 2003;89:228-34.

6. Cecchi M, Landini G, Andreini R, et al. Percorso medico ospedale per intensità di cure. Toscana Medica 2013;31:32-6.

7. Landini G. Trattamento domiciliare della trombosi venosa profonda: percorso assistenziale ospedale-territorio. Ital J Med 2009;3:39-42.

8. Jimenez D, Aujesky D, Moores L et al. Simplification of the Pulmonary Embolism Severity Index for the prognostication in patients with acute symptomatic pulmonary embolism. Arch Intern Med 2010;170:1383-9. 
9. Masotti L, Pieralli F, Panigada G, et al. Characteristics and clinical management of acute pulmonary embolism in real world: findings from TUSCAN-PE Study. Ital J Med 2014. [Epub ahead of print].

10. Masotti L, Lorenzini G, Landini GC, et al. New oral anticoagulants for acute and long term treatment of hae- modinamically stable pulmonary embolism. Global J Respir Care 2014;1:1-8.

11. Nieto JA, Solano R, Iglesias NT, et al. Validation of a score for predicting fatal bleeding in patients receiving anticoagulation for venous thromboembolism. Thromb Res 2013;132:175-9. 


\title{
Governo clinico e Medicina Interna: quali proposte per il prossimo futuro?
}

\author{
Roberto Risicato \\ UOC Medicina Interna, PO Augusta - ASP Siracusa, Italia
}

\section{RIASSUNTO}

Nei sistemi sanitari moderni, si assiste ad un divario fra i bisogni dell'utente, e le risorse economiche utilizzabili. Ciò avviene per una serie di cause, fra cui il cambiamento del contesto socio-economico, la crisi del welfare state, l'invecchiamento demografico della popolazione, la medicina sempre più tecnologica, l'introduzione di metodi di controllo di gestione e la responsabilizzazione, il maggior coinvolgimento del malato e dei familiari nelle scelte cliniche. Tutto ciò comporta la necessità di rendere compatibile efficienza clinica ed efficienza economica. I dati del Ministero della Salute italiano, dicono che negli ultimi 10 anni la spesa sanitaria è cresciuta da 76 a circa 113 miliardi di euro, rappresentando circa il 7,1\% del prodotto interno lordo nazionale. La media europea è di circa $8,2 \%$. È evidente anche in Italia, che bisogna conciliare aspettative di aumento di durata della vita col miglioramento della qualità assistenziale, specie per il malato cronico, con budget sempre più ridotti a disposizione. In particolare, ogni confronto clinico ed economico, che coinvolge i pazienti della Medicina Interna, deve considerare la particolare tipologia di malato. La complessità clinica ed assistenziale da gestire in Medicina Interna, quindi, determina un obbligatorio percorso formativo del medico che si dedichi ad essa, l'internista, il quale deve acquisire competenze multidisciplinari e peculiari doti non solo tecniche, ma anche gestionali e relazionali. Le UO di Medicina Interna rappresentano di fatto organizzazioni ad elevata complessità, cui afferiscono pazienti fra loro assai diversi e con flussi non programmabili. La complessità dei casi trattati è rilevante, sia per la gravità clinica, fragilità, comorbilità e disabilità, sia per i quadri atipici di presentazione. L'idea della clinical governance (CG) affermatasi nel Regno Unito alla fine degli anni novanta, rappresenta un momento ideale di sintesi per governare la complessità delle aziende sanitarie, perseguendo il duplice obiettivo di promuovere la qualità assistenziale e al tempo stesso salvaguardare l'efficienza e la sostenibilità economica. La formazione continua attraverso percorsi codificati e validati, deve essere parte integrante dell'attività delle aziende sanitarie, permettendo un maggiore sviluppo professionale e culturale dei suoi professionisti, cui corrisponderà un vantaggio all'azienda stessa. Alla base della CG vi è la consapevolezza che la prestazione sanitaria è un sistema complesso, che richiede l'intervento di molteplici e differenti professionalità in un percorso articolato. La sua applicazione, richiede la collaborazione fra dirigenti amministrativi e professionisti medici. Riteniamo che questi ultimi siano chiamati ad essere protagonisti e responsabili delle scelte cliniche, ma che debbano anche ampliare diffusamente le competenze in modo da poter controllare efficacemente le dinamiche dei costi senza compromettere la qualità dell'assistenza. Siamo convinti che il contributo al miglioramento delle cure passi dall'integrazione della formazione e della crescita tecnico-professionale con quella gestionale-relazionale. Tutte insieme rappresentano l'imprescindibile bagaglio culturale e professionale del dirigente medico. È compito particolare, e gravoso, dell'internista, e della società scientifica che lo rappresenta, favorire la diffusione e la capillarizzazione di questi concetti e di queste conoscenze.

\section{Introduzione}

Fin dagli anni settanta, l'aumento del costo della salute ha rappresentato un argomento politico di primaria importanza, sia in USA, che nei Paesi Europei.

Corrispondente: Roberto Risicato, UOC Medicina Interna, PO Augusta - ASP Siracusa, Italia.

E-mail: r.risicato@inwind.it

Parole chiave: governo clinico, Medicina Interna, proposte.

Articolo pubblicato secondo la Creative Commons Attribution NonCommercial 3.0 License (CC BY-NC 3.0).

CCopyright R. Risicato, 2014

Licensee PAGEPress, Italy

QUADERNI - Italian Journal of Medicine 2014; 2:148-157
Infatti, negli ultimi 40 anni, sono stati tentati numerosi tentativi, non tutti riusciti, di moderare i costi sanitari, attraverso riforme e approcci manageriali ai problemi della salute dei cittadini. È stato calcolato che in USA, nel 2019, la spesa sanitaria assorbirà il 19,3\% del prodotto interno lordo (PIL) e visto che la spesa sanitaria è data dalla somma dei servizi erogati, moltiplicata per il loro costo, sembra semplice ridurre la spesa riducendo, o i servizi, o i relativi costi, o entrambi. La difficoltà sta, di fatto, non solo nel quantificare i costi dei servizi, ma soprattutto nel definire quali sono le prestazioni sanitarie che uno stato deve erogare al cittadino, definendone il valore, quindi sotto il profilo dell'appropriatezza e dell'efficienza clinica. oltre che economica. Coinvolgendo, infine, la preferenza del paziente e le differenti condizioni cliniche del singolo caso. ${ }^{1}$ Come la nostra sanità: le strategie introdotte per il ridimensionamento della spesa sono state fallimen- 
tari a causa di un forte aumento della domanda di servizi sanitari al fronte di forti sprechi nella gestione dell'offerta - la sanità assorbe il 7,1\% del PIL, aumento della spesa pro-capite, dal 1990 al 2005: 80\%, aumento PIL $37 \%$. Infatti si è registrato un forte aumento della domanda di servizi sanitari, a fronte di forti sprechi nella gestione dell'offerta (Figure 1 e 2).

Questa circostanza è generalmente evidenziata nei sistemi sanitari moderni, in cui si assiste ad un divario fra i bisogni dell'utente, e le risorse economiche utilizzabili. Ciò avviene per una serie di cause che brevemente possono essere riassunte:

- Cambiamento del contesto socio-economico e crisi del welfare state

- Invecchiamento demografico della popolazione

- Paradosso della medicina tradizionale, concepita per le malattie acute, e che deve adattarsi al contesto prevalente di malattie croniche

- Gestione condivisa del paziente complesso fra diversi professionisti, in setting diversi

- Modificazioni della struttura della famiglia

- Medicina sempre più tecnologica (crisi del modello biomedico della malattia)

- Miglior livello culturale della popolazione

- Aumento delle aspettative di salute da parte degli utenti

- Introduzione di elementi di economia aziendale nella gestione della sanità

- Maggiore coinvolgimento del malato e dei familiari nelle scelte cliniche

- Necessità di rendere compatibile efficienza clinica ed efficienza economica

- Cambiamenti sistemici nell'organizzazione del lavoro

- Introduzione di metodi di controllo della gestione e responsabilizzazione

- Ricerca della qualità dell'assistenza e dell'appropriatezza clinica e gestionale. $^{2}$

I dati del Ministero della Salute italiano, dicono che negli ultimi 10 anni la spesa sanitaria è cresciuta

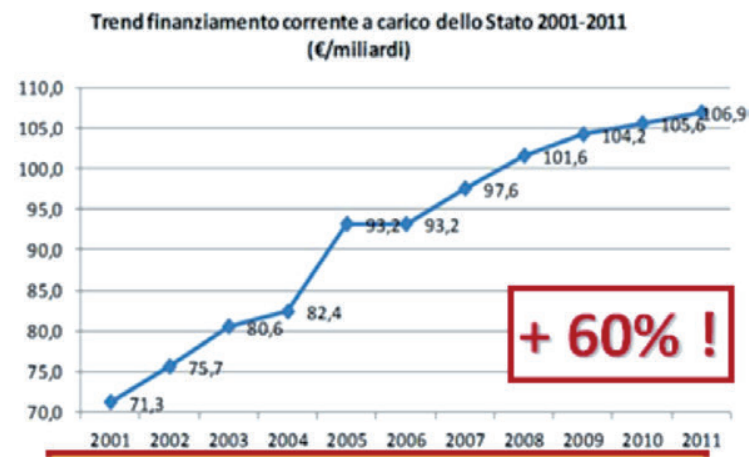

Figura 1. Incremento progressivo della spesa sanitaria in Italia (dati Ministero della Salute). da 76 a circa 113 miliardi di euro, rappresentando circa il 7,1\% del PIL nazionale. La media europea è di circa 8,2\%. È evidente anche in Italia, che bisogna conciliare aspettative di aumento delle aspettative di vita e di miglioramento della qualità assistenziale, specie per il malato cronico, con budget sempre più ridotti a disposizione. Nelle logiche di monitoraggio dei processi delle aziende sanitarie, l'apporto degli studi di economia aziendale si è rivelato nel tempo di cruciale importanza nell'offrire metodi e tecniche di rilevazione, misurazione, e gestione agli operatori sanitari, irrompendo in una cultura estranea ed il più delle volte ostile, come quella medico-clinica, e coinvolgendo progressivamente le diverse categorie di attori presenti nel governo di sistema. ${ }^{3}$

Di fatto, non è del tutto compresa la soluzione della dicotomia costo e qualità della assistenza sanitaria. Si è cercato di risolvere il trade off fra equità ed efficienza attraverso il rapporto costo/beneficio. Di fatto, il problema è più complesso. Il tema dell'appropriatezza delle cure, sempre più trattato e diffuso, sembra poter affrontare e cercare di risolvere il dilemma. Appropriatezza che implica efficienza, efficacia, adeguatezza, correttezza, in relazione a modalità tecnica, allocazione di risorse, produzione di risultati, opportunità sociale ed economica. ${ }^{4}$ Basti pensare all'alta percentuale di pazienti che si rivolgono ai dipartimenti di emergenza, e che potrebbero invece essere curati dal Medico di Medicina Generale: 40\%. ${ }^{5}$ In un contesto in cui assistiamo ad un incremento di ricoveri di pazienti in età senile, che rappresentano il 40\% del totale (con circa il 50\% delle giornate di degenza e dei relativi costi). ${ }^{6}$ E l'incremento nel Regno Unito, fra il 1990 ed il 2004, di pazienti di età superiore ai 70 anni è stato del 198\%, e di età maggiore ai 90 anni, del $671 \%$. $^{7}$ Ogni confronto clinico e d economico, che coinvolge, però, pazienti della Medicina Interna, deve considerare la particolare tipologia di malato, che spesso è anziano, complesso, polipatologico, fragile, politrattato, clinicamente instabile, funzionalmente di-

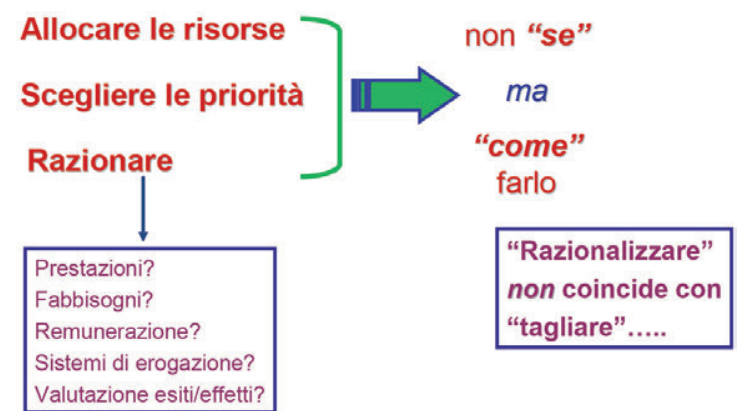

Figura 2. Il problema delle scelte allocative. 
pendente, malnutrito, con carenze socio-economiche e/o affettive (Figure 3 e 4).

È questa la tipologia di malato con cui dobbiamo confrontarci e per la quale dobbiamo prevedere percorsi assistenziali, basati sui principi di buona pratica clinica, guidati dalle Evidenze migliori, possibilmente integrati, nell'ambito della continuità assistenziale, con gli altri setting clinici post-acuzie (Lungodegenza, Riabilitazione, ADI, RSA, Hospice), e con i Servizi Sociali, assieme ai quali, prendere in carico nella sua globalità l'ammalato con quelle caratteristiche. La complessità clinica ed assistenziale da gestire in Medicina Interna, quindi, determina un obbligatorio percorso formativo del medico che si dedichi ad essa, l'internista, il quale deve acquisire competenze multidisciplinari e peculiari doti non solo tecniche, ma anche gestionali e relazionali.

La clinical competence dell'internista richiede necessariamente l'inquadramento delle conoscenze, abilità, capacità tecniche, delle qualità professionali, manageriali, ed operative di ogni singolo specialista nel contesto sanitario di riferimento (Figura 5). ${ }^{8} \mathrm{Se}-$ condo Epstein la competenza del medico richiede un uso ponderato e giudizioso della comunicazione, delle conoscenze, delle abilità tecniche, delle capacità di ragionamento clinico, delle emozioni e dei valori, della capacità di riflessione nella pratica quotidiana, per il bene dei singoli e della comunità. ${ }^{9,10}$ Infatti, la piramide della clinical competence di Miller deve oggi essere inquadrata nel contesto organizzativo della complessità assistenziale dei pazienti trattati, secondo livelli differenti di responsabilità e di caratteristiche professionali: sa, sa come, mostra come, fa. Le diverse tipologie di competenza s'intersecano continuamente nell'attività quotidiana del singolo professionista, quindi devono convivere in lui ${ }^{11,12}$ le competenze e le abilità tecniche (hard skills o competenze core) strettamente intese, devono integrarsi con le cosiddette soft skills, attinenti alla capacità di comunicazione e presentazione, leadership e management, gestione delle risorse umane, project management, customer service, lavoro di gruppo, sviluppo personale.

Il dirigente medico è coinvolto in diversi livelli nella organizzazione sanitaria, tutti importanti, e responsabilizzanti (Figura 6).

Nelle organizzazioni complesse dobbiamo opportunamente parlare di mappa delle competenze, ovvero della rappresentazione delle conoscenze, delle abilità tecniche e capacità comportamentali, necessarie per garantire l'efficacia di una performance, con riferimento allo specifico contesto aziendale, complesso, come complessa è la gestione clinica del malato affetto da multi-morbosità. ${ }^{13}$ Le UO di Medicina Interna rappresentano di fatto organizzazioni ad elevata complessità, cui afferiscono pazienti fra loro assai diversi e con flussi non programmabili (Figura 7). La complessità dei casi

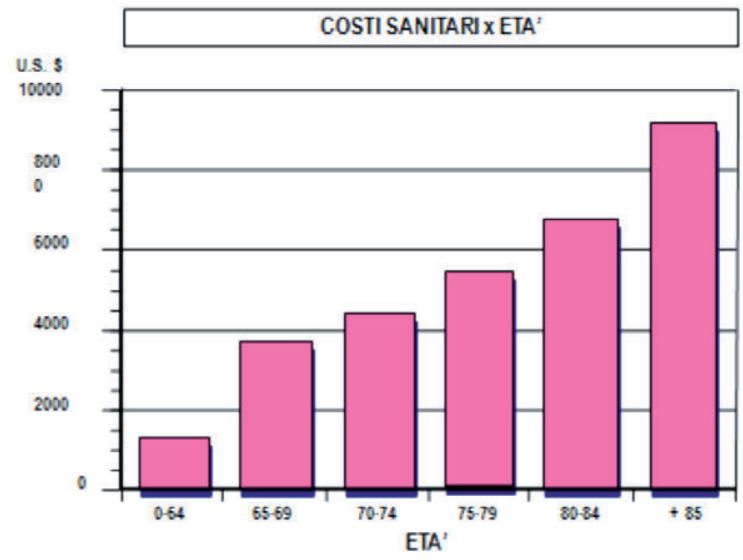

Figura 3. Costi sanitari per età (fonte: The $2^{\text {nd }}$ Pharmaceutical Conference, Sept 1995).

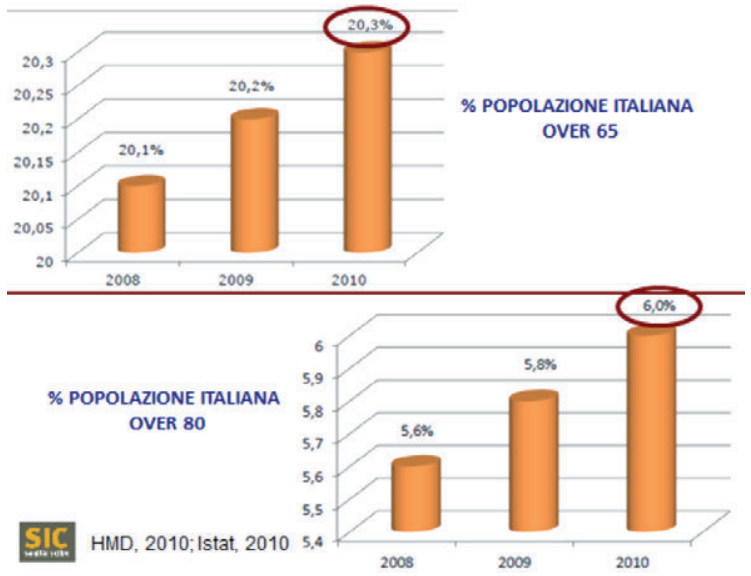

Figura 4. I cambiamenti demografici in Italia.

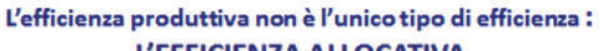
L'EFFICIENZA ALLOCATIVA

"scelta fra impieghi possibili di risorse scarse"

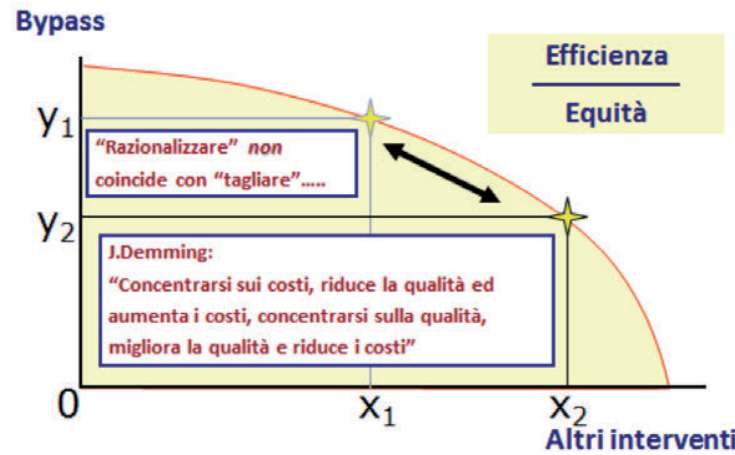

Figura 5. Le scelte allocative obbligate. 
trattati è rilevante, sia per la gravità clinica, fragilità, comorbilità e disabilità, sia per i quadri atipici di presentazione. Si riscontrano, inevitabilmente, incertezze e difficoltà nel delineare percorsi diagnostico-terapeutici. Scienza clinica ed esperienza devono essere complementari, con evidente riferimento al paziente reale, che l'internista deve estrapolare da quello individuato nei trial e nelle metanalisi da cui vengono tratte le linee guida. Inoltre l'Internista deve conciliare la sua autonomia professionale con la necessità di integrazione $\mathrm{e}$ di standardizzazione dei percorsi assistenziali. Grande importanza deve essere data alla capacità di comunicare e di lavorare in equipe, nonché di sapere utilizzare al meglio le tecnologia di informazione e comunicazione, strumenti utili per migliorare i processi di cura nei sistemi a più elevata complessità. ${ }^{14}$ Altro aspetto importante è relativo alla ricerca clinica, che assume di per sé un valore formative ed etico-comportamentale, in quanto abitua il clinico al rigore ed alla coerenza metodologici, favorisce il confronto tra gli operatori coinvolti, rappresenta uno stimolo culturale rivolto allo sviluppo ed all'innovazione, indirizza il medico alle norme della buona pratica clinica secondo regole di trasparenza. ${ }^{8}$ Bisogna, peraltro, creare un legame stretto fra ricerca e formazione, al fine di coinvolgere gli operatori per il miglioramento della qualità assistenziale. La formazione, deve quindi essere programmata ed organizzata in modo tale che rappresenti la base per migliorare la pratica professionale quotidiana, con ricadute positive sul malato in termini di esiti sanitari. Occorre spostare l'attenzione dalla formazione continua, allo sviluppo professionale continuo, inteso come sviluppo di tutte le conoscenze, abilità e capacità comprese nel processo di cura. Un modello ottimale della valutazione della formazione dovrebbe comprendere tutti i livelli di Kirkpatrick (Figura 8): i) gradimento dei partecipanti; ii) conoscenze ed abilità; iii) cambiamenti delle perfor- mance professionali; iv) ricadute positive sullo stato di salute dei pazienti. ${ }^{15}$ Applicando un modello in grado di integrare l'educazione continua in Medicina nella pratica clinica quotidiana, si impara ad affrontare i problemi correnti della clinica. Questo modello, che si fonda su 4 momenti [i) individuazione dei bisogni formativi; ii) formazione ed apprendimento; iii) utilizzo delle informazioni; iv) azioni di rinforzo], rappresenta un percorso di eccellenza e si inserisce appieno nel concetto di CG, intesa come processo finalizzato ad implementare un sistema di qualità che tenda a migliorare gli esiti sanitari ottimizzando l'uso delle risorse disponibili.

È evidente infine, che il momento formativo diventa elemento strategico del sistema, promuovendo e supportando la crescita professionale. ${ }^{16}$ Quindi preso atto che l'internista deve possedere hard e soft skills, e che la formazione deve essere sistemica, strutturata e crea le basi per migliorare gli esiti sanitari, lo sforzo di una

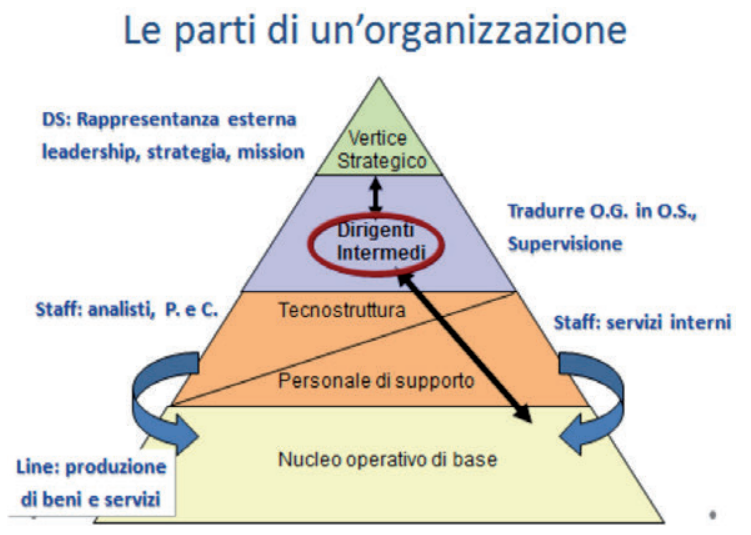

Figura 6. Le parti di una organizzazione professionale: i Dirigenti intermedi.

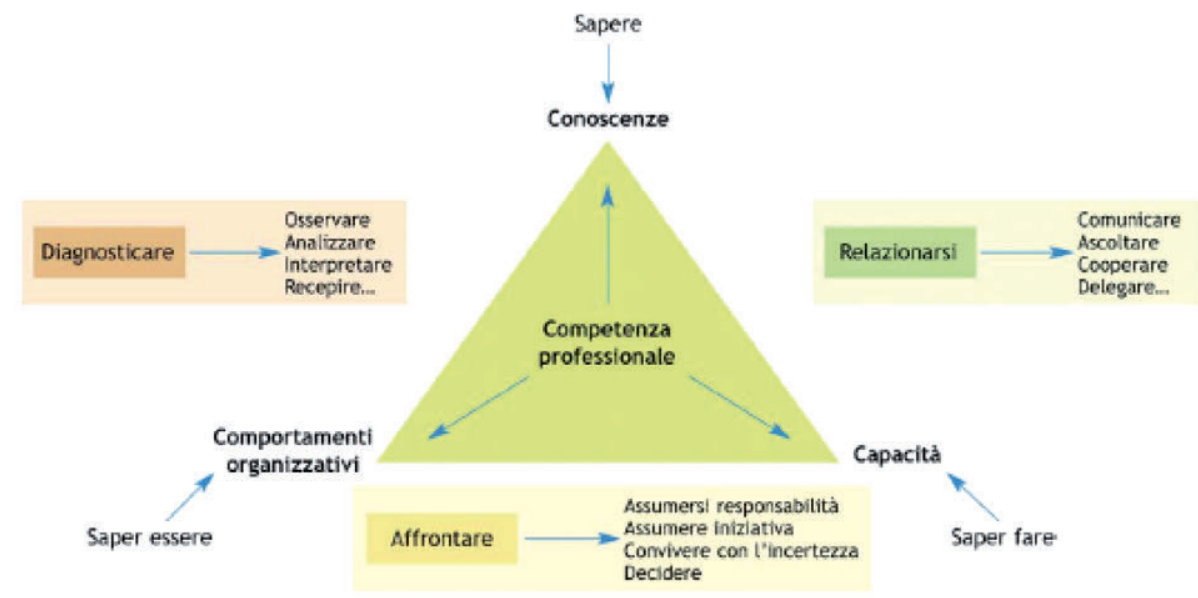

Figura 7. La competence dell'internista (Ital J Med, 2011). 
società scientifica di internisti deve essere quello di implementare percorsi formativi che includano conoscenze e competenze in ambito di CG, che permettono di integrare le abilità tecniche, pratiche e scientifiche, prettamente orientate alla clinica. Nella migliore integrazione fra adeguate e sempre migliori competenze tecniche, gestionali, e relazionali, si realizza la professionalità e la peculiarità dell'internista. I tratti distintivi della Medicina Interna si fondano sui seguenti elementi: i) pluripotenzialità, intesa come capacità di sviluppare ed integrare conoscenze e competenze multiple; ii) flessibilità, che consiste nella capacità di adattare e modificare le priorità di intervento sia nel singolo paziente sia in risposta alle necessità epidemiologiche del territorio; iii) interdipendenza funzionale, nella consapevolezza che il case-mix dei pazienti in carico richiede una dipendenza reciproca con diversi interlocutori, ma al tempo stesso necessita di una regia unitaria rispetto al singolo caso; iv) economicità, che richiede un'efficiente gestione dei posti-letto, meno costosi di quelli con indirizzo di alta specializzazione, e con un differente turn over determinato dal ricovero di pazienti con problematiche non risolte e complesse, per la multi-morbilità e per le interazioni di ordine fisiopatologico, clinico e socio-assistenziale. L'internista è quindi un professionista in continua formazione, con compiti complessi, con un ruolo organizzativo a valenza multidimensionale, che ha importanti ricadute di carattere gestionale oltre che clinico. ${ }^{17}$

\section{Il Governo clinico del sistema sanitario}

Garantire una buona qualità assistenziale è un compito arduo e prioritario dei servizi sanitari moderni, indipendentemente dal grado di complessità tecnologica ed organizzativa che riescono ad esprimere. Le aspettative degli utenti e degli operatori, infatti, sono notevolmente aumentate negli anni. ${ }^{18} \mathrm{Nel}$ contempo, le molteplici ed articolate richieste assistenziali, soprattutto nell'ambito della cronicità, obbligano a scelte e risposte multispecialistiche e multi professionali, da parte di operatori e servizi diversi, che si devono integrare in percorsi assistenziali complessi. Inoltre, le nuove tecnologie, le innovazioni, che devono integrarsi nei piani di cura, richiedono un continuo aggiornamento professionale, ed un miglioramento del rapporto fra ricerca e pratica clinica. Contribuiscono alla qualità dell'assistenza specifiche scelte di politica sanitaria che intervengono sugli assetti organizzativi dei servizi in modo da creare una effettiva ed efficiente multidisciplinarietà integrata: Il trasferimento della conoscenza nella pratica, e la possibilità di documentare i risultati danno, infine, al sistema nel suo insieme un ulteriore contributo verso la qualità. La qualità dell'assistenza è quindi il risultato dell'interazione di tre elementi fondamentali: i) formazione dei professionisti; ii) organizzazione dei servizi e delle loro relative relazioni funzionali; iii) legame ricerca-pratica clinica.

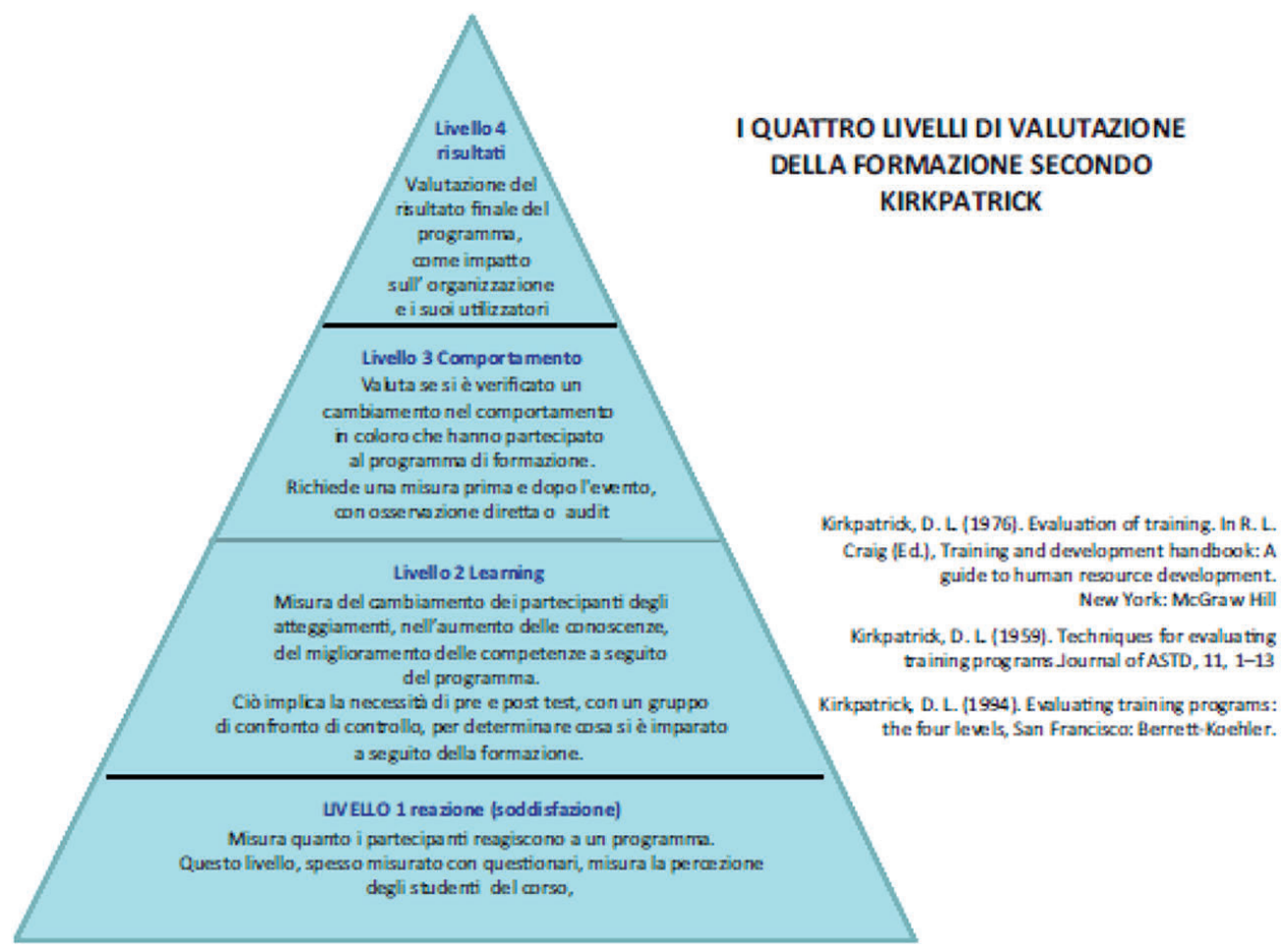

Figura 8. Triangolo di Kirkpatrick (Ital J Med, 2011). 


\section{Total quality management ed evidence based medicine}

Sul versante del miglioramento della qualità, si sono sviluppati due filoni distinti fra loro sul piano operativo e dei presupposti culturali: i) un approccio organizzativo-manageriale (che rivolge scarsa attenzione per il contenuto clinico delle prestazioni sanitarie), che predilige l'efficienza operativa all'efficacia ed all'appropriatezza, prevedendo in campo sanitario, l'applicazione di modelli di organizzazione e controllo ampiamente sperimentati in aziende di settori diversi come la contabilità direzionale, l'activity-based costing, la bilance score card, e il total quality management ${ }^{19}$ ii) un approccio medico-tecnico, finalizzato ad intervenire sulle decisioni cliniche al fine di orientarle verso una maggiore razionalità in termini di efficacia ed appropriatezza delle prestazioni e dei percorsi assistenziali, e che ha trovato la sua espressione nell'applicazione della evidence-based medicine e della evidence-based health care, i cui obiettivi consistono nel razionalizzare il processo decisionale delle Aziende sanitarie. ${ }^{20,21}$

In questo contesto di contrapposizione culturale e metodologica, l'idea della CG affermatasi nel Regno Unito alla fine degli anni novanta, ha ricevuto una favorevole accoglienza, come momento ideale di sintesi per governare la complessità delle aziende sanitarie, perseguendo il duplice obiettivo di promuovere la qualità assistenziale e al tempo stesso salvaguardare l'efficienza e la sostenibilità economica. ${ }^{20}$ Secondo il National Health Service, il GC è il contesto in cui $i$ servizi sanitari si rendono responsabili del miglioramento continuo della qualità dell'assistenza e sostengono elevati livelli prestazionali, creando un ambiente favorevole all'espressione dell'eccellenza clinica, nel rispetto del vincolo delle risorse disponibilli. ${ }^{22} \mathrm{~L}$ 'idea della CG sviluppata dagli anglosassoni faceva pensare alla struttura che trasformava in un occhiale i due monocoli. ${ }^{23}$ In questo modo, restituisce da un lato una visione complessiva di insieme di approccio alla qualità dell'assistenza, e dall'altro fornendo lenti nuove in grado di allentare la tensione dicotomica e le resistenze ai due approcci legate a specifiche difficoltà applicative di alcun strumenti. Il GC fornisce infatti una nuova chiave di lettura di strumenti che del tutto nuovi non sono. ${ }^{24}$ Alla base del GC vi è la consapevolezza che la prestazione sanitaria è un sistema complesso, che richiede l'intervento di molteplici e differenti professionalità in un percorso articolato e soggetto a modifiche per effetto della rapida evoluzione di conoscenze e tecnologie. Il risultato è un complesso intreccio di fattori, e richiede la collaborazione fra dirigenti amministrativi e professionisti medici. Questi ultimi sono chiamati ad essere non solo protagonisti e responsabili delle scelte cliniche, ma anche attori chiave nella definizione, nel controllo e nella valutazione dei risultati. Ancora più importante è il ruolo che deve essere assunto dai medici, in quanto sono gli unici professionisti dotati delle competenze necessarie per esercitare un efficace controllo sulle dinamiche dei costi senza compromettere la qualità dell'assistenza. Questa dimensione rende inevitabilmente il dirigente medico un cultural chamaleos con una attività, una specializzazione, un corredo di competenze (hard e $s o f t$ ) e di abilità, che richiedono una formazione specifica, continua, sistematica, e determinano nella professione medica un boundary spanning. Si è creata la figure, negli anni, del medico manager che rappresenta il connubio delle due figure: medico e manager (Figure 9 e 10).

Questo proprio perché il GC ha come obiettivo il

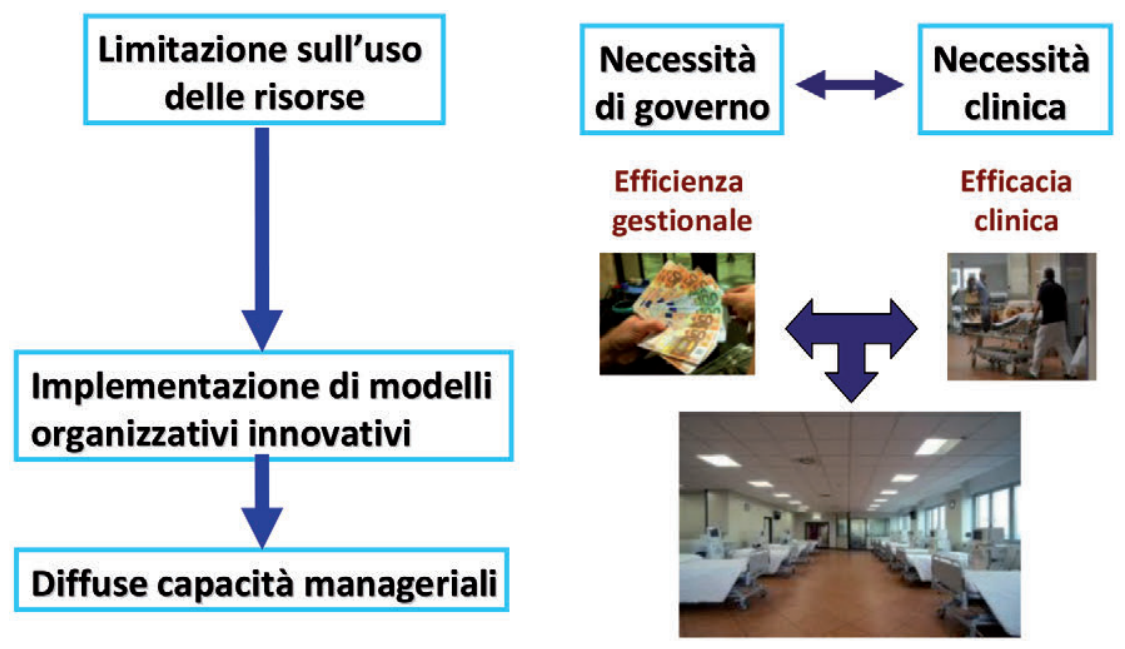

Figura 9. Il medico-manager come sintesi di due culture molto diverse. 
miglioramento della qualità dell'assistenza, non può non avere una spiccata attenzione all'uso delle informazioni scientifiche ed alla gestione della conoscenza come riferimento per le decisioni cliniche, al fine di orientare verso una maggiore efficacia ed appropriatezza clinica. Il governo della pratica clinica implica anche un'esplicita responsabilizzazione degli operatori, che si esprime attraverso la disponibilità ad operare il monitoraggio delle proprie prestazioni, e la capacità di migliorare la comunicazione fra professionisti, e con gli utenti, ai quali chiedere maggiore coinvolgimento, collaborazione e condivisione delle scelte. Un adeguato piano di sviluppo di GC deve prevedere un approccio che includa cultura, knowledge management, che sono fra gli elementi riassunti nell'acronimo: SPOCK (Struttura, Processo, Outcome, Cultura, Knowledge management). E visto che il GC si propone di determinare le relazioni e sviluppare gli strumenti operativi che permettano l'interazione tra le varie componenti professionali, il medico, che svolge il ruolo centrale nell'organizzazione sanitaria, deve considerarsi all'altezza, ed integrare le sue attività assistenziali e di ricerca, con quelle organizzativo-gestionali e relazionali, nella consapevolezza che la formazione in ambito di GC rappresenta uno strumento che serve a migliorare la qualità assistenziale, strumento che altrimenti resta ad esclusiva gestione della componente amministrativa dell'azienda, creando un gap culturale nel medico, lasciandolo fuori dalle scelte e dai momenti decisionali, cioè ghettizzandolo nel ruolo marginale di tecnico esecutore di prestazioni assistenziali condizionate da scelte e politiche sanitarie decise altrove, da burocrati e manager di ben altra formazione culturale.

\section{La formazione continua}

In un contesto quale quello sanitario, caratterizzato da attività sempre più articolate, organizzazioni complesse, continua innovazione ed introduzione di tecnologie e conoscenze scientifiche, specializzazione sempre più spinta, la formazione continua si pone quale strumento fondamentale per mantenere gli adeguati livelli di conoscenza e di skill del personale sanitario e garantire l'elevato livello di qualità delle performance cliniche. Definita come un processo di apprendimento continuo...che migliora gli esiti di salute...ed espande e completa le potenzialità dei professionisti, essa è tanto più necessaria in quelle organizzazioni che si basano sul lavoro dei professionisti, come, appunto, quelle sanitarie. Queste sono caratterizzate dalla prevalenza, tra i meccanismi di coordinamento, della standardizzazione delle capacità, o delle competenze. La formazione, nell'ambito delle burocrazie dei professionisti, conferisce un valore aggiunto alla qualità dell'assistenza, consentendo, non solo di indurre mutamenti comportamentali che abbiano ripercussioni dirette o indirette sull'efficacia delle singole prestazioni sanitarie, ma anche di favorire la condivisione della crescita professionale all'interno dei setting assistenziali in cui il know how del professionista effettivamente esercita.

La formazione continua attraverso percorsi codificati e validati, deve essere parte integrante dell'attività non solo delle aziende sanitarie, permettendo un maggiore sviluppo professionale e culturale dei suoi professionisti, cui corrisponderà un vantaggio all'azienda stessa. Questa breve premessa ci permette di introdurre il concetto di people strategy. L'attenzione alla risorsa umana, mediante azioni e programmi di gestione e sviluppo delle risorse umane, deve intervenire sulla vision e sulla cultura dell'azienda, traducendosi e assicurando la trasformazione dei comportamenti organizzativi in coerenza con i fattori fondamentali di successo per la generazione di valore di scambio tra organizzazione e persona. Il termine people strategy sostiene la dimensione people come parte integrante della strategia di business che genera valore puntando sulle specificità delle risorse a disposizione in grado di creare vantaggio competitivo, cioè facendo leva su competenze essenziali per raggiungere un vantaggio sostenibile sulla concorrenza: è il superamento della più tradizionale politica delle risorse umane (orientata a soddisfare i bisogni individuali quali identità, stabilità, sviluppo) (Figura 11). ${ }^{25}$

Anche le Società scientifiche, devono farsi carico di questa specifica formazione, e dell'implementazione di percorsi formativi fortemente caratterizzati per una istruzione di tipo manageriale, improntata alla diffusione delle conoscenze in ambito di GC. Rivolgendo l'attenzione, non solo ai Direttori di Unità Operativa, o di Dipartimento, ma anche ai dirigenti, meglio se giovani, e che possano acquisire, secondo un percorso parallelo apprendere le abilità e competenze medico-tecniche, quelle medico-manageriali. Assumendo come principio di fondo, e diffondendo la convinzione che solo una maggiore competenza di

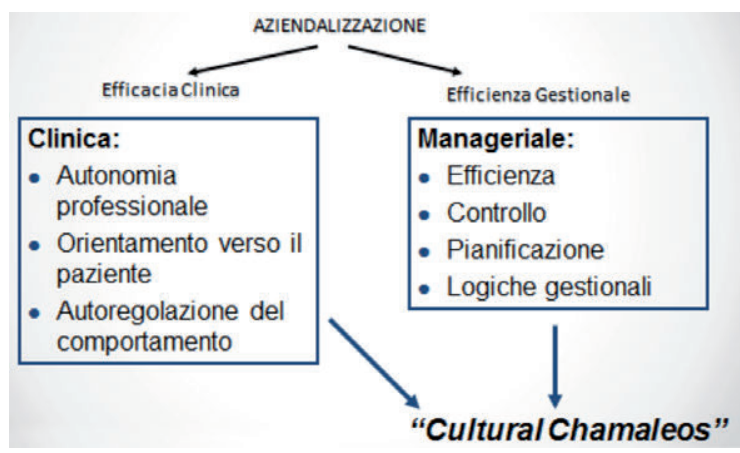

Figura 10. Il medical management. 
tipo manageriale, una maggiore diffusione ed applicazione dei principi del GC permettono di migliorare la qualità dell'assistenza, e, in ultima analisi, quindi, rappresentano un aspetto diverso delle caratteristiche peculiari del medico ospedaliero.

La struttura del GC è stata rappresentata sotto forma di schemi e semplificazioni grafiche di indubbia chiarezza. L'albero di Chamber rimane fra le più suggestive e complete. In esso l'anatomia del GC viene illustrata sottoforma di un albero i cui rami costituiscono le discipline (branches) del modello. Richiamando la Figura 1 già presentata nel Capitolo di Davide Croce a pagina 83 di questa monografia, ritroviamo: i) politica/sistemi di gestione per la qualità; ii) medicina, pratica e policy basate sull'evidenza; iii) health technology assessment; iv) pratica costante di audit e verifica; v) sistemi di accountability; vi) management del rischio (risk management).

Le radici sono rappresentate da: i) affidabilità dei dati (reliable data); ii) responsabilizzazione nella gestione delle risorse (managing resources and service); iii) organizzazione basata sull'apprendimento (learning culture); iv) ricerca e sviluppo (research and development).

Secondo modelli geometrici, la struttura del GC contiene: i) formazione continua (Education); ii) clinical audit; iii) clinical effectiveness; iv) risk management; v) research and development; vi) trasparenza (openness).

A questi elementi il Dipartimento della Qualità del Ministero della Salute aggiunge: i) la gestione dei reclami e del contenzioso; ii) la comunicazione e la gestione della documentazione; iii) la collaborazione multidisciplinare; iv) il coinvolgimento dei pazienti; v) la valutazione del personale; vi) gli esiti. ${ }^{20}$

In particolare, per le premesse sopra espresse, l'internista è lo specialista ospedaliero che maggiormente si presta a diventare l'esperto, per la soluzione dei problemi gestionali complessi che i casi clinici nella pratica quotidiana pongono: pazienti anziani, fragili, malnutriti, con multimorbosità, con problemi familiari, sociali, con la complessità clinica ed assistenziale che caratterizza il malato di Medicina Interna. L'internista, medico della complessità deve impegnarsi nell'acquisire le competenze gestionali e relazionali, di pari passo a quelle tecniche, per tornare ad incarnare la figura del medico di riferimento per gli altri specialisti, secondo un percorso formativo continuo incentivato dalle attività formative e di confronto della società scientifica degli internisti ospedalieri (FADOI), che deve farsi carico di guidare questa presa di coscienza e questa rivoluzione culturale, che l'internista deve affrontare pur nelle innumerevoli difficoltà che giornalmente incontra.

Se applichiamo al Progetto CG e Medicina Interna l'analisi SWOT, che permette di identificare i punti di forza (strenghts), le debolezze (weakeness), le possibilità (opportunities) e i pericoli (threats), vedremo come numerosi fattori intervengono in modo antitetico per favorire od ostacolare la diffusione della CG nelle aziende sanitarie italiane (Figure 12-14). I principali artefici di questa rivoluzione culturale devono essere proprio i sanitari, condannati, altrimenti, a subirla passivamente, da colpevoli spettatori, mentre hanno la concreta possibilità, apprendendo tecniche, modalità, linguaggio, percorsi, di rappresentare lo snodo principale del processo, gli artefici del cambiamento, con un ruolo attivo prioritario. Naturalmente tutto ciò richiederà del tempo. L'internista rappresenta, di certo, lo specialista che deve farsi carico prima e più degli altri specialisti, di intraprendere questa strada.

Nella organizzazione sanitaria moderna, la coesistenza delle due competenze dicotomiche deve essere diffusa in modo capillare, e rappresenta la reale modalità per rendere più efficace il processo di miglioramento continuo delle cure.

In questo percorso, la società scientifica, deve svolgere un ruolo formativo importante, attraverso iniziative didattiche face-to-face, nelle diverse e più efficaci modalità, per ampliare le conoscenze teoriche, ma anche attraverso il training pratico, ed il bedside teaching che permettano acquisizione di nozioni pratiche, scambi di esperienze, formazione sul campo, ed una diffusione capillare delle competenze hard e skill (Figura 15).

\section{Conclusioni}

Il report del 2012 di Bain and Company, società di consulenza, evidenzia come in tutti i sistemi sanitari nazionali delle economie mondiali più avanzate nei prossimi 10 anni assisteremo a più cambiamenti di quanti ne abbiamo visto negli ultimi 50 anni. Si prevede un incremento della spesa sanitaria pubblica che andrà dal valore di $0,6 \%$ del PIL in Italia, al 1,5\% della Francia, del 3,3\% nel Regno Unito, del $6 \%$ circa in USA (qui avrà un peso

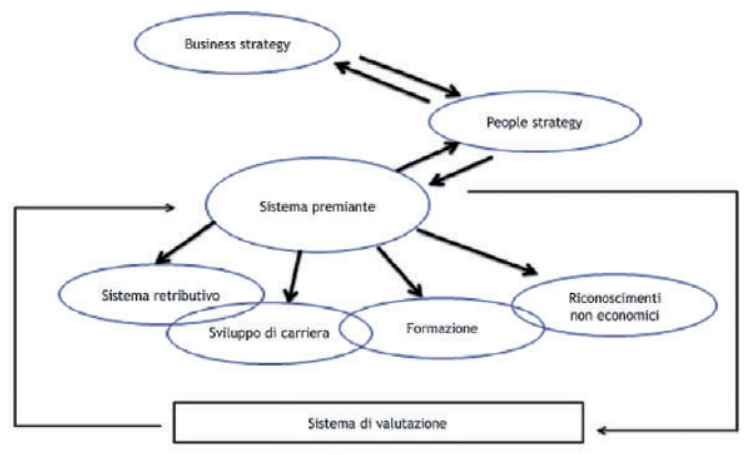

Figura 11. Business strategy e people strategy. 
spesso fra l'incudine del Pronto Soccorso/MCAU/Area dell'Emergenza, ed il martello del Post-acuzie/Territorio, entrambe caratterizzate da inefficienze e criticità organizzative, carenze di personale e di supporto tecnico. Il ruolo di parafulmine nelle situazioni di maggiore complessità e criticità, ha permesso però, nel tempo, all'internista, di affinare quelle competenze cliniche e gestionali, e quella elasticità culturale, che lo rendono idoneo al ruolo di guida per questo processo innovativo.

Crediamo sia necessario svecchiare concetti, comportamenti, pregiudizi, abitudini. Questo passaggio richiede un grande salto culturale. Si tratta di un percorso difficile ed accidentato che merita la massima attenzione e preparazione, intelligenza e impegno, assorbe energie, a tutti noi impegnati quotidianamente ad affrontare le difficoltà organizzative e, soprattutto, la sofferenza e la malattia, che abbiamo scelto di conoscere e combattere. Siamo convinti che il contributo al miglioramento delle cure passi dall'integrazione della formazione e della crescita tecnico-professionale con quella gestionale-relazionale. Insieme rappresentano l'imprescindibile bagaglio culturale e professionale del dirigente medico. È compito particolare, e gravoso, dell'internista, e della società scientifica che lo rappresenta, favorire la diffusione e la capillarizzazione di questi concetti e di queste conoscenze. Affiancando le Aziende sanitarie, ed il Ministero della Salute, nel percorso di formazione continua. Questa è la sfida, la proposta, per il prossimo futuro.

\section{Bibliografia}

1. McMahon LF, Chopra V. Health care and cost value, the way forward. JAMA 2012;307:671-2.

2. Nardi R. Quale appropriatezza per la decisione clinica in medicina interna? Ital J Med 2008;2:1-3.

3. Russo S. L'ABC e il benchmarking di processo nella valutazione delle attività di day surgery. MECOSAN 2011; 80:19-38.

4. Nardi R, Berti F, Fabbri LM, et al. Toward a sustainable and wise healthcare approach: potential contributions from hospital Internal Medicine Departments to reducing inappropriate medical spending. Ital $\mathrm{J}$ Med. 2013;7:65-81.

5. Heneghan C. General practice, p 623. In: Cook S. Experts' guide to saving money in health. BMJ 2010;340: c1281.

6. Ministero della Salute. Stato di salute e prestazioni sa- nitarie nella popolazione anziana - anno 2000. Roma: Ministero della Salute; 2003.

7. George G, Jell C, Todd BS. Effect of population ageing on emergency department speed and efficiency: a historical perspective from a district general hospital in the UK. Emerg Med J 2006;23:379-83.

8. Mathieu G, Greco A, Nardi R, et al. La clinical competence in Medicina Interna. Ital J Med 2011;5:S17-29.

9. Medical Professionalism Project. Medical professisonalism in the new millennium: a physicians' charte. Lancet 2002;359:520-2.

10. Epstein RM, Hundert EM. Defining and assessing professional competence. JAMA 2002;2872:226-35.

11. Miller GE. The assessment of clinical skills/competence/ performance. Acad Med 1990;65:S63-7.

12. Norcini JJ Work based assessment. BMJ 2003; 326 (7392):753-5.

13. Associazione Italiana per la Formazione Manageriale. Glossario ASFOR. Riv Assoc Ital Formaz Manage 2006; XVIII(1 suppl).

14. Stevens DP. Quality and safety are rarely simple. Qual Saf Health Care 2009;182:82-3.

15. Kirkpatrick DL. Evaluating training programs: the four levels. San Francisco: Berrett-Koehler; 1994.

16. Heard SR, Schiller G, Aitken M, et al. Continuous quality improvement: educating towards a culture of clinical governance. Qual Health Care 2001;10:ii70-8.

17. Nardi R, et al. Modelli di valutazione della clinical competence del medico specialista internista ospedaliero. Ital J Med 2011;5:S33-45.

18. Groll R, Baker R, Moss F. Quality improvement research: under standing the science of change in health care. London: BMJ Books; 2004.

19. Bruno P. La competenza manageriale in sanità: i nuovi modelli di responsabilità in una sanità che cambia. Milano: Ed. Franco Angeli; 2008.

20. Capasso A, Pascarella G. L'Azienda sanitaria: innovazione tecnologica, evoluzione organizzativa e governo clinico. Milano: Ed. Franco Angeli; 2011.

21. Sackett DL, Rosenberg WM, Gray JA, et al. Evidence based medicine: what it is and what it isn't. BMJ 1996; 312:71-2.

22. Fontana F. Clinical Governance: una prospettiva organizzativa e gestionale Milano: Ed. Franco Angeli; 2005.

23. Buetow SA, Roland M. Clinical Governance: bridging the gap between managerial and clinical approaches to quality care. Qual Health Care 1999;8:184-90.

24. Freeman T. Measuring progress in clinical governante: assessing the reliability and validity of the clinical governance climate questionnaire. Health Serv Manage Res 2003;16:234-50.

25. Filannino C, Stornello I, Nardi S. Le politiche di people strategy in sanità: o sviluppo delle clinical competence per la crescita professionale del dirigente medico. Ital J Med 2011;5:S2-16. 


\title{
Potenzialità dell'audit clinico in Medicina Interna: lo studio DDIMA
}

\author{
Maurizia Gambacorta, in collaborazione con il DDIMA Group \\ UOC Medicina Interna, Ospedale Media Valle del Tevere, Todi (PG), Italia
}

\begin{abstract}
RIASSUNTO
L'audit clinico è uno strumento di clinical governance $(\mathrm{CG})$ che abbiamo a disposizione per verificare e migliorare la qualità dell'assistenza, in particolare l'appropriatezza di processi. Come emerge dalla definizione del National Health Service del 1996 si tratta di una revisione tra pari, strutturata, per mezzo della quale i professionisti esaminano la propria attività e i propri risultati a confronto con standard concordati e la modificano se necessario. Perché risulti efficace va programmato e condotto secondo le varie fasi: i) pianificazione dell'audit; ii) selezione di criteri, indicatori e standard; iii) confronto della pratica con gli standard; iv) identificazione degli scostamenti dagli standard e feedback; v) implementazione del cambiamento; vi) re-audit per verificare l'effettivo cambiamento, illustrate nel dettaglio di seguito. Nella trattazione viene portato ad esempio un'esperienza di audit multicentrico condotta all'interno dell'Area Permanente FADOI per la CG, che ha come oggetto la dimissione del paziente diabetico dalle UO di Medicina Interna. Questa esperienza dimostra come l'audit sia uno strumento utile quando applicato alla realtà della Medicina Interna e quali possono essere le sue potenzialità per ulteriori applicazioni.
\end{abstract}

\section{Introduzione}

L'audit clinico si inserisce nella cornice della clinical governance $(\mathrm{CG})$, costituita da numerose attività integrate, come il principale strumento per verificare e migliorare la qualità dell'assistenza, in particolare l'appropriatezza di processi.

A 60 anni di distanza dal suo ingresso negli ospedali anglosassoni le sue caratteristiche principali non sono mutate: si tratta di una valutazione strutturata e sistematica, condotta da professionisti sulla propria pratica professionale, attraverso un confronto con criteri espliciti, al fine di ottenere un miglioramento delle cure.

Nel corso degli anni si sono però spostati alcuni accenti: l'audit è diventato multi professionale (da medical audit a clinical audit) e con l'introduzione dell'evidence-based medicine si è trasformato in uno strumento indispensabile per confrontare la pratica dei

Corrispondente: Maurizia Gambacorta, UOC Medicina Interna, Ospedale Media Valle del Tevere, Todi (PG), Italia. E-mail: mauriziagambacorta@yahoo.it

Parole chiave: audit clinico, dimissione paziente diabetico, continuità assistenziale paziente diabetico.

Articolo pubblicato secondo la Creative Commons Attribution NonCommercial 3.0 License (CC BY-NC 3.0).

CCopyright M. Gambacorta et al., 2014

Licensee PAGEPress, Italy

QUADERNI - Italian Journal of Medicine 2014; 2:158-165 professionisti con le evidenze scientifiche e le raccomandazioni che ne derivano.

\section{Definizione}

Sulla base delle indicazioni fornite da un gruppo di studio del Governo Britannico del 1996, l'audit clinico può essere definito come una iniziativa condotta da professionisti che cerca di migliorare la qualità e gli esiti dell'assistenza attraverso una revisione tra pari, strutturata, per mezzo della quale i professionisti esaminano la propria attività e i propri risultati a confronto con standard concordati e la modificano se necessario.

Il termine audit trova la propria radice etimologica nel verbo latino audire: udire, ascoltare, apprendere, interpretare. Il termine medical audit viene introdotto nel 1956 da P.A. Lembcke nell'accezione di una revisione sistematica ed una valutazione scientifica delle cartelle cliniche, per verificare i risultati delle pratiche clinico-assistenziali. Il medical audit comincia ad entrare in Gran Bretagna all'inizio degli anni ' 70 , ma la spinta decisiva alla sua diffusione arriva alla fine degli anni ' 80 . Nel novembre 1992 si riunisce per la prima volta il clinical outcome group, composto da medici e infermieri e dal 1993 si inizia a parlare di audit clinico comprendendo anche il contribuito di altre professioni sanitarie alla qualità delle cure. ${ }^{1}$

\section{Caratteristiche dell'audit clinico}

Dalla stessa definizione emergono in maniera molto netta le caratteristiche fondamentali dell'audit clinico: i) è un'iniziativa a carattere professionale, ba- 
sata su di una competenza clinico-assistenziale specifica dei partecipanti ed avente come oggetto un ambito fortemente legato alla qualità professionale; ii) è un'attività continua e sistematica e non può quindi essere limitata alla revisione di singoli casi; iii) ha come oggetto principale l'appropriatezza dei processi, consentendo di misurare il grado di inappropriatezza (in eccesso o in difetto) e di identificare quali aree della pratica clinica devono essere oggetto di miglioramento; iv) deve prevedere la possibilità di accedere ad una adeguata documentazione clinica nonché la verifica della buona pratica corrente rispetto a standard noti; v) i risultati emersi sono assolutamente confidenziali: non ha alcuna finalità ispettiva e di controllo. ${ }^{2}$

\section{Il ciclo dell'audit clinico}

Il processo dell'audit clinico viene in genere rappresentato graficamente come un ciclo, costituito da 6 passaggi chiave (Figura 1$)^{3}$ : i) pianificazione dell'audit; ii) selezione di criteri, indicatori e standard; iii) confronto della pratica con gli standard; iv) identificazione degli scostamenti dagli standard e feedback; v) implementazione del cambiamento; vi) re-audit per verificare l'effettivo cambiamento.

Ad ogni ciclo successivo dell'audit si mira a raggiungere target di qualità sempre più elevati. ${ }^{3}$
All'interno dell'area permanente FADOI per la CG è stata condotta un'esperienza di audit multicentrico, che va sotto l'acronimo di DDIMA (Diabetic patients discharge from Internal Medicine Audit). Riportiamo questa esperienza quale esempio di applicazione dell'audit ad una problematica clinica, al fine di mostrare quali possono essere le potenzialità di questo strumento quando applicato nel contesto della Medicina Interna.

\section{Fase 1. Pianificazione dell'audit}

Rappresenta una fase fondamentale per la buona riuscita dell'audit clinico. Questa fase preliminare prevede: i) la scelta del tema dell'audit clinico; ii) la costituzione del gruppo di lavoro; iii) la definizione di popolazione, intervento e obiettivo; iv) la ricerca, selezione e validazione delle evidenze; v) la definizione del campione statistico; vi) la creazione della scheda di raccolta dati e del relativo database; vii) la definizione delle modalità di feedback.

Analizziamo nel dettaglio i primi due punti che rappresentano un elemento cruciale per la buona riuscita delle fasi successive dell'audit clinico.

\section{Scelta del tema}

In letteratura vengono indicate in maniera abbastanza omogenea le priorità che possono guidare questa scelta: i) alti volumi: frequenza dell'attività; ii) alti

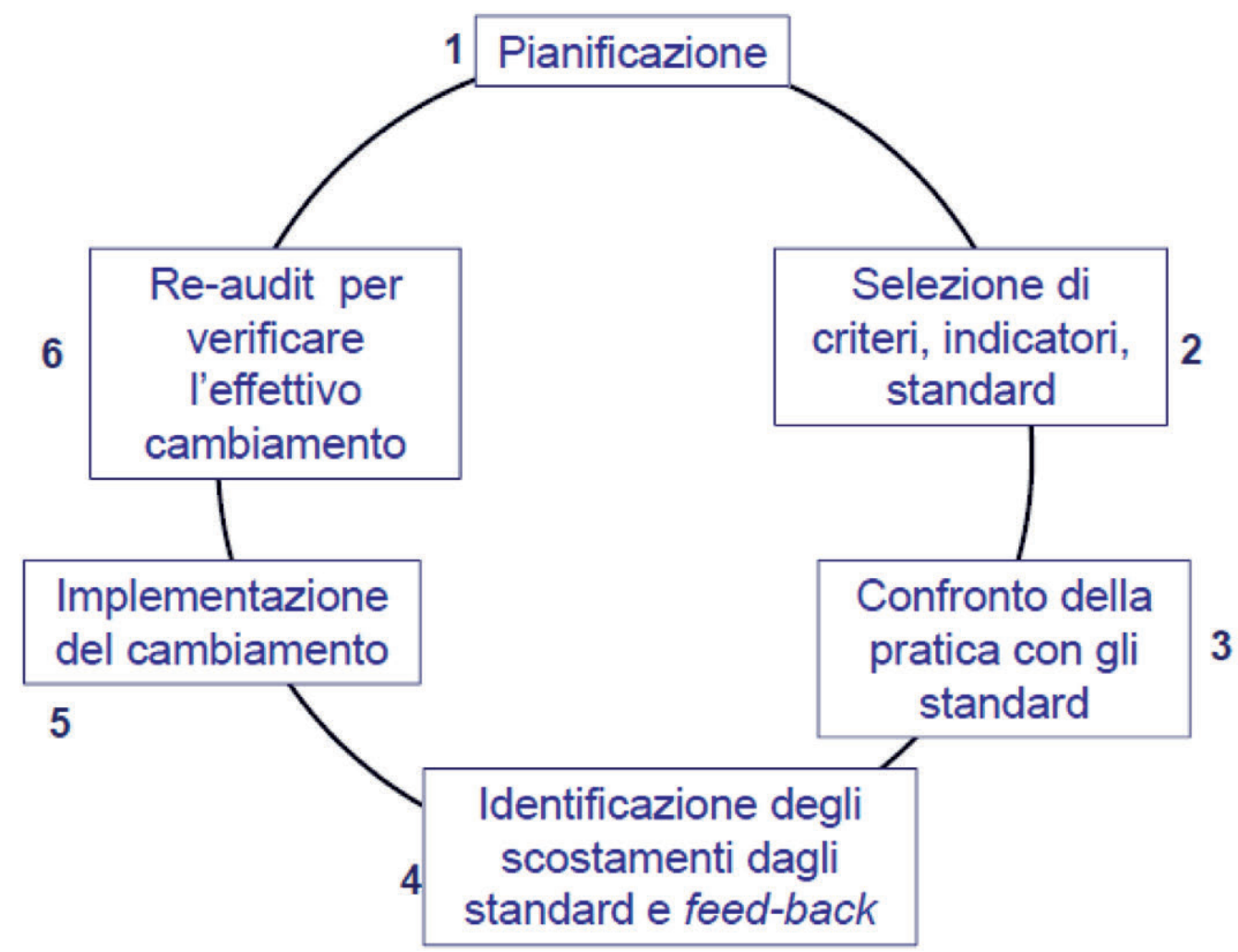

Figura 1. Il ciclo dell'audit clinico (modificato da Benjamin, 20083). 
costi: processi ad elevato impegno di risorse; iii) alta rischiosità: criticità dell'attività per i pazienti e per gli operatori; iv) alta variabilità: eterogeneità; v) alta complessità: prestazioni ad elevato livello clinico-assistenziale ed organizzativo; vi) alto contenuto innovativo: health technology assessment. ${ }^{4}$

La scelta del tema dell'audit non può prescindere dalla disponibilità in letteratura di evidenze di grado elevato con cui confrontare la pratica clinica.

È fondamentale che la priorità definita, oltre a richiamare l'interesse dei professionisti fornendo loro un'opportunità di sviluppo professionale, sia rilevante per l'organizzazione sanitaria, rispondendo agli obiettivi di miglioramento della qualità, nonché per il cittadino/paziente, per cui rappresenta una garanzia di efficacia e sicurezza delle cure. ${ }^{5}$

\section{Costituzione del gruppo di lavoro}

All'interno del gruppo di lavoro devono essere rappresentate tutte le competenze necessarie per effettuare un'analisi approfondita del processo di cura o del particolare problema individuato. A tale scopo il team dovrà essere multidisciplinare, comprensivo di tutte le discipline coinvolte nel processo assistenziale, e multiprofessionale, al fine avere una rappresentanza di tutte le professioni interessate. In genere la dimensione di un team di audit non dovrebbe superare le 1012 persone, ma per piccole strutture possono essere sufficienti 4 o 5 persone. All'interno del gruppo è importante individuare un leader, un professionista con conoscenza del metodo ed esperienza nella gestione degli audit clinici, in grado di guidare il cambiamento e con buone capacità di comunicazione.

Inoltre è utile il coinvolgimento della direzione aziendale per sostenere il cambiamento, soprattutto nelle fasi in cui vengono definite le azioni di miglioramento.

Nonostante finora sia stata del tutto marginale, è auspicabile la partecipazione dei pazienti, non solo perché l'esperienza dei pazienti è un elemento fondamentale per valutare la qualità delle cure, ma anche perché i pazienti stessi costituiscono la migliore rete informativa in merito alle iniziative di miglioramento

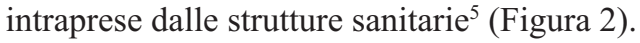

Il Gruppo di lavoro del DDIMA è costituito da 20 Internisti appartenenti ad altrettante UO di Medicina Interna distribuite su tutto il territorio nazionale: si tratta infatti di un audit multicentrico.

Un gruppo ristretto di professionisti ha inizialmente selezionato 10 argomenti che poi, applicando il metodo quality impact analysis, ha condiviso con l'intero gruppo (60 elementi): da qui è stato selezionato l'argomento che ha riscosso più interesse in base a livello di frequenza, rischio e criticità.

Il tema scelto è stato la dimissione del paziente diabetico dalle UO di Medicina Interna, poiché si aveva coscienza di u problema legato alla scarsa attenzione agli aspetti educazionali e al continuum assistenziale. L'obiettivo che si è posto il gruppo è stato quello di migliorare la continuità assistenziale dei pazienti diabetici tra ospedale e territorio.

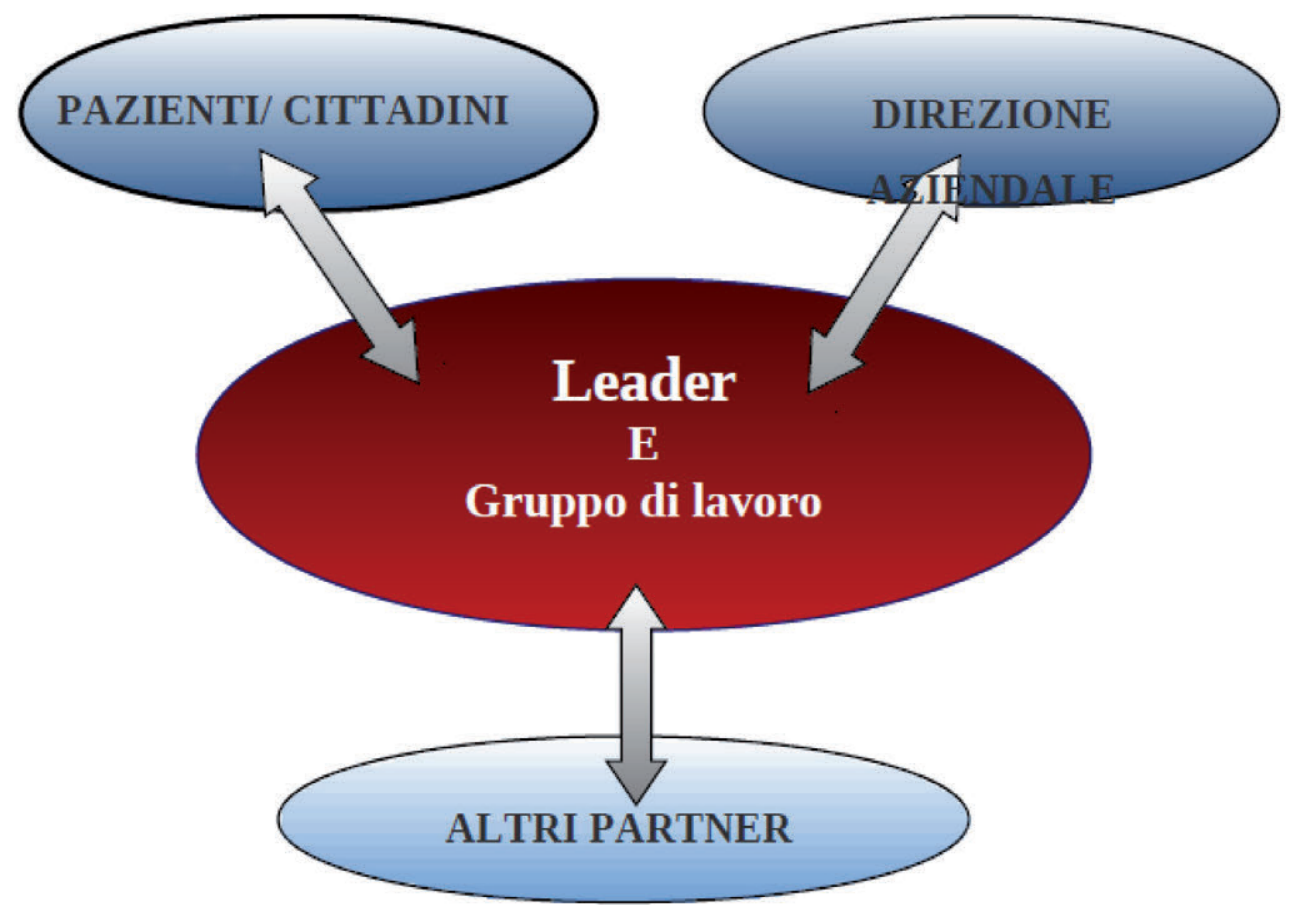

Figura 2. Il gruppo di lavoro dell'audit clinico (Ministero della Salute, 20115). 
La scelta del tema rispondeva sicuramente alla priorità alti volumi: basti pensare che si stima che almeno un paziente su 4 tra quelli ricoverati è diabetico. Sicuramente soddisfatta era anche la priorità relativa agli alti costi. Il diabete è una patologia molto costosa, arrivando ad assorbire circa 1' $8 \%$ della spesa del Servizio Sanitario Nazionale (SSN) (costi sociali), ma nei costi diretti vanno annoverati anche $i$ costi individuali (spesa personale non coperta dal SSN); accanto a questi abbiamo anche i costi indiretti, a loro volta classificabili come costi indiretti tangibili (assenza dal lavoro con mancato guadagno, impegno dei familiari, ecc.) e costi indiretti intangibili (disabilità, qualità e quantità della vita).

Alcuni dati forniti recentemente dal Ministero della Salute stimano che un paziente diabetico costa in un anno circa 4 volte di più di un paziente con analoghe caratteristiche ma non diabetico e che la maggior parte di queste risorse viene assorbita dai ricoveri ospedalieri. ${ }^{6}$

Infine il tema scelto si presta bene alla realizzazione di un audit in quanto le evidenze scientifiche disponibile sono numerose e di elevato livello: un gruppo ristretto di 5-6 professionisti ha raccolto e selezionato la letteratura relativa all'argomento scelto.

\section{Fase 2. Selezione di criteri, indicatori e standard}

Il criterio è un aspetto definibile e misurabile dell'assistenza sanitaria che ne descrive la qualità e può essere usato per valutarla. È la definizione esplicita di ciò che si deve fare e si deve ottenere in una determinata situazione. Un criterio dovrebbe quindi essere: i) evidence-based; ii) condiviso tra $\mathrm{i}$ professionisti; iii) pertinente, cioè correlato ad aspetti rilevanti dell'assistenza; iv) traducibile in indicatore; v) misurabile.

Si ricava da linee-guida, percorsi diagnostico terapeutici ed assistenziali (PDTA), evidenze scientifiche di buona qualità, normative. ${ }^{7}$

L'indicatore è una informazione o una variabile selezionata che consente di descrivere fenomeni complessi e misurare variazioni in relazione a criteri definiti, allo scopo di orientare le decisioni volte ad ottenere o mantenere cambiamenti. In altre parole è un parametro misurabile che indica quanto nella pratica clinica ci si avvicina all'applicazione del criterio specifico. Viene espresso sotto forma di percentuale, tasso o media.

Gli indicatori possono essere relativi a: i) struttura: riguardano le risorse logistiche, strumentali ed umane; ii) processo: si riferiscono ai processi di cura, ai processi decisionali. Sono i più utilizzati nell'audit clinico; iii) risultato: mirano a misurare l'impatto dei processi sull'evoluzione o sullo stato clinico dei pazienti, a rilevare l'esperienza dei pazienti, a valutare i costi.

Lo standard è il valore soglia (standard minimo o ottimale) a cui l'indicatore deve tendere a sotto al quale non deve scendere.

Lo standard può essere: i) determinato dalla letteratura scientifica internazionale; ii) determinato da indicazioni di società scientifiche; iii) ottenuto da altre strutture sanitarie, ritenute autorevoli e competenti (benchmark); iv) calcolato su rilevazioni effettuate in periodi precedenti.

Lo standard deve essere realistico e raggiungibile, standard del $100 \%$ sono quasi sempre utopistici. ${ }^{8}$

In base alle evidenze raccolte l'intero gruppo dei partecipanti alo studio ( 20 centri) ha selezionato i criteri, costruito gli indicatori ed identificato i relativi standard.

Gli standard italiani per la cura del diabete mellito 2009-2010 recitano: ....un intervento educativo su alcuni aspetti essenziali, quali le modalità di iniezione dell'insulina e i principi dell'autocontrollo, devono essere forniti al diabetico prima della dimissione (livello della prova IV, forza della raccomandazione B). Partendo da questa raccomandazione, che rappresenta il nostro criterio, si è proceduto alla costruzione di due indicatori di processo (Tabella 1).

\section{Indicatori di processo e standard del DDIMA}

Gli indicatori di processo e standard del DDIMA sono i seguenti: i) $\mathrm{N}^{\circ}$ di pazienti che ricevono istruzioni per l'autocontrollo glicemico $/ \mathrm{N}^{\circ}$ di pazienti diabetici dimessi: $80 \%$; ii) $\mathrm{N}^{\circ}$ di pazienti che ricevono istruzioni per il trattamento dell'ipoglicemia $/ \mathrm{N}^{\circ}$ di pazienti diabetici dimessi in trattamento farmacologico: $80 \%$; iii) $\mathrm{N}^{\circ}$ di pazienti che ricevono istruzioni per la somministrazione di insulina/ $\mathrm{N}^{\circ}$ di pazienti diabetici dimessi in trattamento insulinico: $90 \%$; iv) $\mathrm{N}^{\circ}$ di pazienti dimessi con consigli nutrizionali o dieta personalizzata/ $\mathrm{N}^{\circ}$ di pazienti diabetici dimessi: $80 \%$; v) $\mathrm{N}^{\circ}$ di pazienti diabetici o con iperglicemia dimessi con

Tabella 1. Indicatori di processo.

\begin{tabular}{llc}
\hline Indicatore di processo & Valutazione appropriatezza & Standard \\
\hline Numeratore & $\mathrm{N}^{\circ}$ pazienti che ricevono istruzioni per la somministrazione dell'insulina & $90 \%$ \\
Denominatore & $\mathrm{N}^{\circ}$ pazienti dimessi in terapia insulinica & \\
\hline Numeratore & $\mathrm{N}^{\circ}$ pazienti che ricevono istruzioni per autocontrollo domiciliare & $80 \%$ \\
Denominatore & $\mathrm{N}^{\circ}$ pazienti diabetici dimessi & \\
\hline
\end{tabular}


appuntamento ambulatoriale $/ \mathrm{N}^{\circ}$ di pazienti diabetici o con iperglicemia dimessi: $80 \%$; vi) $\mathrm{N}^{\circ}$ di pazienti con valore dell'emoglobina riportata in lettera di dimissione $/ \mathrm{N}^{\circ}$ di pazienti diabetici o con iperglicemia dimessi: $90 \%{ }^{9}{ }^{9-12}$

\section{Fase 3. Confronto della pratica con gli standard}

In questa fase si realizza la raccolta dei dati, che può essere effettuata in maniera prospettica o retrospettiva. In quest'ultimo caso viene esaminata la documentazione clinica del passato: è un metodo più veloce, ma spesso la qualità della documentazione clinica risulta insoddisfacente al fine della raccolta dei dati. Raccogliere dati da fonti diverse - per esempio sistemi informatici per la gestione degli esami di laboratorio e radiologici - può contribuire a risolvere il problema.

Può essere utile predisporre una scheda costruita appositamente per la raccolta delle informazioni, una check-list di audit. È necessario definire criteri di inclusione ed esclusione dei pazienti, oltre ai criteri da esaminare nell'audit e il loro periodo di applicazione.

I dati così raccolti devono quindi essere analizzati al fine di stabilire, per ciascun criterio stabilito, se gli standard sono stati rispettati. A questo punto è necessario analizzare quanto la prassi corrente si discosti dallo standard ed identificare eventuali ipotesi di miglioramento.

Tutti i centri che partecipavano al DDIMA hanno revisionato almeno 60 cartelle in maniera retrospettiva, esaminando a ritroso le cartelle cliniche di tutti pazienti dimessi con diagnosi di diabete mellito in maniera consecutiva, fino al raggiungimento del campione stabilito.

Sono stati revisionati un totale di 1332 casi di pazienti diabetici dimessi dalle UO di Medicina Interna di tutta Italia. Per agevolare questa fase era stata predisposta una scheda raccolta dati cartacea.

I dati sono stati raccolti in un unico database e analizzati sia nella loro totalità che in analisi di sottogruppo in base a parametri di patologia ed organizzazione (Figura 3).

\section{Fase 4. Identificazione degli scostamenti dagli standard e feedback}

In questa fase si confrontano $\mathrm{i}$ dati raccolti con $\mathrm{i}$ criteri e gli standard, si valuta in che misura gli standard siano stati soddisfatti e si identifica il divario, criterio per criterio, tra la pratica clinica e lo standard prestabilito. A questo confronto deve far seguito una analisi approfondita delle cause dei divari, che può essere effettuata con alcuni strumenti come il brainstorming, il diagramma di causa-effetto, l'albero delle cause, il diagramma di Pareto.

Le cause responsabili del divario tra pratica clinica e standard possono essere di 3 tipi: i) professionali: mancanza di conoscenze, abilità, ragionamento, forza dell'abitudine; ii) organizzative: insufficiente coordinamento delle cure; iii) strutturali: mancanza o inadeguatezza delle strutture.

A questo punto è indispensabile fornire un feedback a tutti i soggetti coinvolti nell'audit. Questo si realizza attraverso il rapporto dell'audit, che dovrebbe includere: i) la presentazione del metodo; ii) il referenziale per la valutazione e il protocollo dell'audit; iii) i risultati, la relativa analisi e gli scostamenti; iv) una descrizione precisa delle situazioni di non conformità rilevate; v) le aree di miglioramento e le raccomandazioni.

È importante sottolineare che la riunione finale dell'audit clinico non è un rito bensì il miglior mezzo per convincere i valutati della necessità di migliorare.

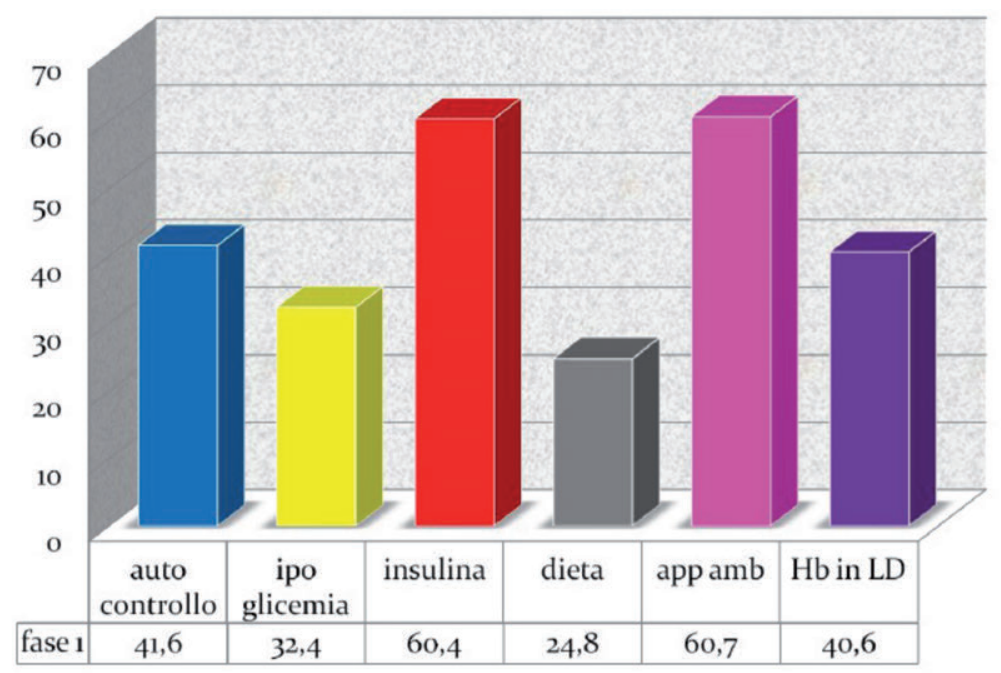

Figura 3. Prima fase di raccolta dei dati. 
Il gruppo dei partecipanti al DDIMA ha quindi deciso le possibili aree di miglioramento, gli strumenti da mettere in campo ed il timing per l'implementazione e la revisione dei cambiamenti.

All'interno di ogni UO è stato condotto un audit interno in cui i risultati della prima fase sono stati condivisi, così da fornire un feedback a tutto il personale medico e infermieristico; sono state individuati gli scostamenti, analizzate le possibile cause e proposte le soluzioni da adottare per colmare il divario tra pratica clinica e standard.

\section{Fase 5. Implementazione del cambiamento}

Il percorso di miglioramento può essere affrontato con diversi approcci: i) approccio di processo: migliorare un percorso trasversale o un segmento del percorso assistenziale, aggiornare, elaborare, validare le procedure assistenziali e i protocolli terapeutici; ii) approccio di risultato: migliorare gli esiti valutati attraverso opportuni indicatori; iii) approccio di risorse: introduzione di nuovi materiali e risorse.

Quindi nel caso in cui abbiamo individuato cause di tipo professionale, agiremo attraverso un approccio di processo e di risultato, che prevede l'educazione del personale con meeting interattivi, l'utilizzo di reminders, l'introduzione di pay for performance.

Anche nel caso in cui le cause siano di tipo organizzativo dovremmo indirizzare la nostra azione di miglioramento verso un approccio di risultato e di processo, attraverso per esempio la costruzione e l'implementazione di PDTA.

Infine nel caso in cui le cause siano di tipo strutturale sarà necessario mettere in atto un approccio di risorse: negoziazione di risorse umane, finanziare e materiali con la Direzione.
È raccomandabile stabilire azioni, responsabili, tempi di implementazione e modalità di monitoraggio.

Il gruppo dei partecipanti al DDIMA ha ideato e implementato degli strumenti per ottimizzare il momento della dimissione del paziente diabetico, al fine di rendere questo passaggio un continuum con il territorio e non un momento di distacco.

È stato adottato un opuscolo con alcune informazioni essenziali da consegnare al paziente dopo aver fornire oralmente tutte le istruzioni necessarie ed è stata creata una check-list da inserire in cartella, per evitare di omettere qualcuno degli interventi previsti.

\section{Fase 6. Re-audit}

Consiste nell'effettuare una nuova misurazione degli indicatori con gli stessi strumenti e le stesse modalità operative al fine di rendere i risultati confrontabili.

Se l'audit aveva evidenziato un divario significativo solo su alcuni criteri, il re-audit potrà focalizzarsi soltanto sui criteri risultati fortemente deficitari.

Laddove questi comportino un rischio elevato per i pazienti, l'azione di miglioramento dovrà essere immediata, così come rapida dovrà essere la rivalutazione dei criteri.

Dopo 3 mesi dall'implementazione degli strumenti in ogni UO sono state esaminate 80 cartelle cliniche relative ai pazienti dimessi con diagnosi di diabete mellito, utilizzando la stessa modalità retrospettiva usata nella prima fase. La Figura 4 mostra i risultati della seconda fase a confronto con quelli della prima fase: nonostante gli standard che ci eravamo prefissati non siano stati raggiunti si è avuto un netto miglioramento della percentuale di tutti gli indicatori.

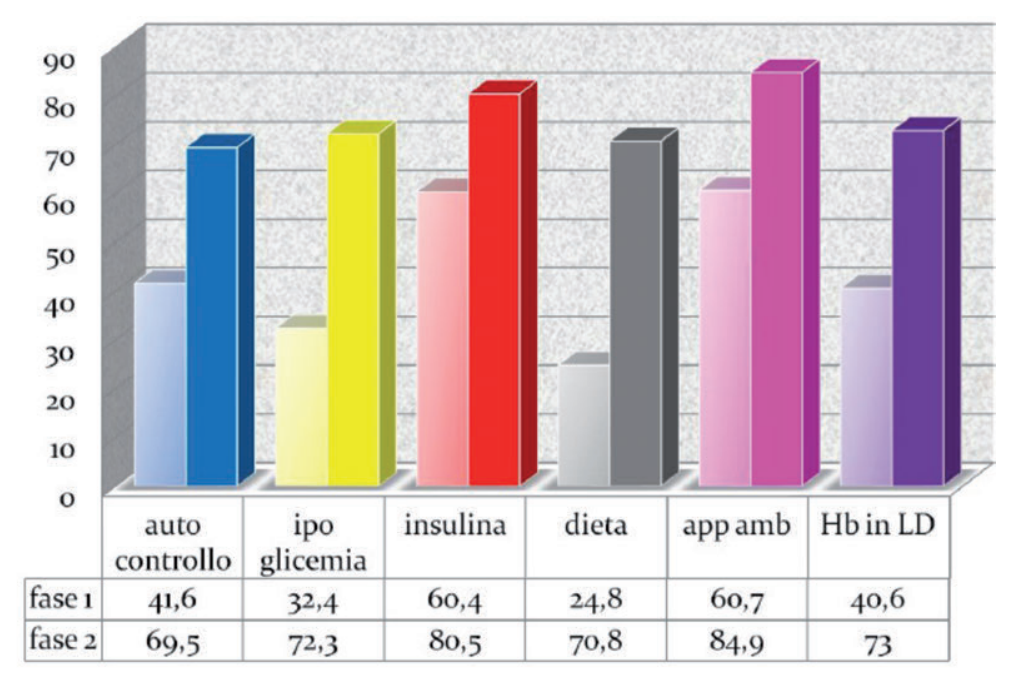

Figura 4. Confronto tra prima e seconda fase di raccolta dei dati. 


\section{L'audit funziona?}

Vi sono evidenze discordanti sul fatto che l'audit funzioni. L'audit e il feedback non si sono dimostrati costantemente efficaci. Una recente revisione sistematica ha concluso che fornire agli operatori sanitari dati relativi alla loro performance sotto forma di audit e feedback può contribuire a migliorare la loro pratica assistenziale. La revisione ha concluso che l'efficacia relativa di un audit e del corrispondente feedback era probabilmente maggiore quando l'aderenza iniziale ad una pratica raccomandata era bassa e quando il feedback era effettuato con maggiore intensità.

Nonostante questi dati non così incoraggianti, l'audit rimane il miglior strumento disponibile per misurare la pratica assistenziale e per sapere quando dobbiamo modificarla. ${ }^{13,14}$

L'esperienza del DDIMA conferma quanto emerso da questa revisione; infatti ad eccezione di alcune eccezioni, sia in positivo che in negativo, si è assistito ad un omogeneo incremento della percentuale di tutti gli indicatori, quindi ad un complessivo miglioramento della qualità dell'assistenza fornita.

I risultati migliori si sono ottenuti laddove le performances iniziali erano particolarmente basse e laddove le condizioni organizzative erano maggiormente favorevoli al cambiamento (medici più giovani, maggiori competenze gestionali, ecc.).

\section{Conclusioni}

A tutt'oggi le esperienze riportate in letteratura riguardanti audit clinici condotti all'interno di UO di Medicina Interna rappresentano una minoranza, pari al 3\% di tutte le pubblicazioni sugli audit.

Questo dimostra che il potenziale dell'audit in Medicina Interna rimane ancora in gran parte da sfruttare.

Alla base di questo sottoutilizzo dell'audit clinico in Medicina Interna potrebbero esserci ragioni legate al particolare setting di pazienti che afferiscono alle nostre $\mathrm{UO}$, per lo più pazienti complessi e pluripatologici. Se infatti da un lato il setting ideale per la realizzazione di un audit clinico prevede una popolazione omogenea, la disponibilità di evidenze di alta qualità per la definizione di criteri e standard, una certa omogeneità degli operatori per formazione, esperienza, interessi e la disponibilità di strumenti per la rilevazione degli indicatori (registri di malattia, database), dall'altro lato il setting della medicina interna è caratterizzato da una popolazione disomogenea, dalla disponibilità di evidenze di alta qualità metodologica non sempre trasferibili nella real life, da operatori con formazione, esperienza, interessi diversi, criteri non sempre univocamente applicabili, motivazione dell'aderenza all'audit variabile, difficoltà di comunicazione tra operatori e as- senza di strumenti per la rilevazione degli indicatori.

Nonostante queste limitazioni le potenzialità dell'audit clinico, che la nostra piccola esperienza ha cercato di dimostrare, dovrebbero spingerci ad usarlo con sempre maggiore frequenza, in quanto i suoi vantaggi consistono in: i) migliorare la pratica clinica, producendo reali benefici nella cura del paziente e nella fornitura dei servizi; ii) sviluppare l'apertura al cambiamento; iii) fornire garanzie sulla qualità dell'assistenza mediante applicazione delle migliori pratiche evidence-based.

Gli eventuali vantaggi possono quindi riguardare: i) la persona assistita, poiché favorisce la fiducia nella sicurezza e qualità della cura erogata; ii) gli operatori sanitari, poiché permette loro di acquisire nuove competenze e riconoscimento professionale; iii) l'istituzione, poiché inserisce metodi di gestione e di miglioramento della qualità trasferibili ad altre situazioni.

\section{Bibliografia}

1. Geddes da Filicaia M. Guida all'audit clinic. Roma: Pensiero Scientifico Editore; 2008.

2. Cartabellotta A. Audit clinico: questo sconosciuto (II). Metodologie di pianificazione e conduzione. GIMBEnews 2009;6:4-5. Disponibile a: http://www.evidence.it/ articolodettaglio/209/it/134/leggi/articolo

3. Benjamin A. Audit: how to do it in practice. BJM 2008;336:1241-5.

4. Perraro F, Wienand U, Ravera L, et al. Raccomandazioni sull'audit clinico. Scegliere il tema. Milano: Società Italiana per la Qualità dell'Assistenza Sanitaria (SIQuASVRQ); 2008. Disponibile a: http://www.siquas.it/ attachments/Documenti/Raccomandazioni/R_8Audit/R Audit_Clinico_sceltatema.pdf

5. Ministero della Salute. L'audit clinico; 2011. Disponibile a: http://www.ospfe.it/il-professionista/audit-clinico/audit -clinico/C_17_pubblicazioni_1552_allegato.pdf

6. Bevere F, Dotta F, Fava A, et al. Appropriatezza clinica, strutturale, tecnologica e operativa per la prevenzione, diagnosi e terapia dell'obesità e del diabete mellito. Quaderni del Ministero della Salute 2011;10:1299. Disponibile a: http://www.quadernidellasalute. it/download/download/10-luglio-agosto-2011-quaderno.pdf

7. Ranocchia D, Wienand U, Deales A, et al. Raccomandazioni sull'audit clinico. Scegliere e formulare i criteri in un audit clinico. Milano: Società Italiana per la Qualità dell'Assistenza Sanitaria (SIQuAS-VRQ); 2008. Disponibile a: http://www.siquas.it/attachments/Documenti/Raccomandazioni/R_8Audit/R_Audit_Clinico_cr iteri.pdf

8. Wienand U, Ranocchia D, Deriu P, et al. Raccomandazioni sull'audit clinico. Definizioni: che cosa è l'audit clinico. Milano: Società Italiana per la Qualità dell'Assistenza Sanitaria (SIQuAS-VRQ); 2008. Disponibile a: http://www.siquas.it/attachments/Documenti/ Raccomandazioni/R_8Audit/R_Audit_Clinico_definizione.pdf 
9. Associazione Medici Diabetologi - Società Italiana di Diabetologia. Standard italiani per la cura del diabete mellito 2009-2010. Torino: Infomedica; 2010.

10. American Diabetes Association. Standards of medical care in diabetes. Diabetes Care 2012;35:S11-S63.

11. Beltramello G, Manicardi V, Trevisan R. Trialogue. La gestione dell'iperglicemia in area medica. Istruzioni per l'uso. Ital J Med 2012;6:123-32.

12. ARS Toscana. Progetto mattoni/matrice dell'Agenas; 2012. Disponibile a: https://www.ars.toscana.it/it/aree- dintervento/problemi-di-salute/malattie-croniche/dati-estatistiche/941-il-progetto-mattonimatrice-dellagenasmaggio-2012.html

13. Sylvia J. Hysong. Meta-analysis: audit and feedback features impact effectiveness on care quality. Med Care 2009;47:356-63.

14. Jamtvedt G, Young JM, Kristoffersen DT, et al. Audit and feedback: effects on professional practice and health care outcomes. Cochrane Database Syst Rev 2003;(3): CD000259.

\section{APPENDICE}

\section{DDIMA (Diabetic patients discharge from Internal Medicine Audit) Group}

Tiziana Attardo, Davide Brancato, Mariangela Di Lillo, Alessandro Franco, Alberto Grassi, Massimiliano Loreno, Ada Maffettone, Andrea Montagnani, Laura Morbidoni, Luca Moriconi, Paola Novati, Vincenzo Nuzzo, Antonella Paradiso, Antonella Perretti, Roberta Re, Roberto Risicato, Ilario Stefani, Francesca Talarico, Daniela Tirotta 


\section{Conclusioni: Medicina Interna domani}

Andrea Fontanella

Dipartimento di Medicina, Ospedale del Buon Consiglio Fatebenefratelli, Napoli, Italia

Il primo articolo di questo numero monografico, di Micaela La Regina e Paola Gnerre, ci fa comprendere quanto sia difficile trovare una giusta e completa definizione della Medicina Interna oggi. Per avere la possibilità di disegnare gli ipotetici scenari del domani della Medicina Interna, e della gestione della salute in generale, non si può non partire dalla precisa consapevolezza della realtà attuale e dalla conoscenza dell'evoluzione dell'assistenza sanitaria in Italia e in Europa.

Sempre più frequentemente, nell'ambito della Sanità pubblica ci si trova ad affrontare un processo di cambiamento, sia di tipo procedimentale (modifiche dell'iter, dei requisiti di accesso, passaggi di competenze, modalità di retribuzione, etc.) che di tipo organizzativo (decentramento, contratto di lavoro, controllo di gestione, trasparenza e accesso, etc.). Questo cambiamento normativo è solo un sintomo evidente di un processo evolutivo molto più ampio: la Pubblica Amministrazione è chiamata a ripensare il suo ruolo nella collettività perché la distanza tra istituzioni e cittadini rischia di diventare insostenibile. A tal proposito non si possono trascurare i seguenti punti storici: dal secondo dopoguerra vi è stato un progressivo allontanamento dagli obiettivi misurabili. La spesa pubblica è stata considerata come motore di sviluppo economico. Di conseguenza lo strumento di tale fattore è stato un'articolazione burocratica fine a se stessa. Con l'avvento dell'Unione Europea e la globalizzazione dei mercati vi è stata una saturazione dei sistemi economici occidentali. Diviene, quindi, indispensabile razionalizzare la gestione dell'assistenza sanitaria per poterla rendere fattibile e competitiva. È, inoltre, necessario puntare alla soddisfazione dei bisogni di tutti gli attori che concorrono allo svi-

Corrispondente: Andrea Fontanella, Dipartimento di Medicina Ospedale del Buon Consiglio Fatebenefratelli, via Manzoni 220, 80123 Napoli, Italia.

Cell.:+39.335.653.4737

E-mail: andreafontanella52@gmail.com

Articolo pubblicato secondo la Creative Commons Attribution NonCommercial 3.0 License (CC BY-NC 3.0).

CC Copyright A. Fontanella, 2014

Licensee PAGEPress, Italy

QUADERNI - Italian Journal of Medicine 2014; 2:166-168 luppo dell'assistenza: personale sanitario medico, paramedico, amministrativo, cittadini.

Questa valutazione storica dell'evoluzione socio economica pone l'accento sulla competitività dei sistemi sanitari, non solo come attrattiva sull'utenza, ma anche verso gli stessi operatori. Negli ultimi cinque anni, in Italia, su 6-7000 laureati in Medicina circa il $2 \%$ è emigrato all'estero in cerca di lavoro. Un numero considerevolmente sottostimato, si considera, infatti, che circa mille giovani dottori ogni anno varchino il confine italiano. Francia, Germania, Svezia, Norvegia, Regno Unito, Stati Uniti e Australia i paesi più gettonati.

Mentre da un lato vengono a ridursi le forze giovani per il nostro Sistema Sanitario, dall'altro la nostra popolazione invecchia sempre di più: in Italia ci sono circa 16.000 ultracentenari, e 3,6 milioni sono gli ultraottantenni. Nella Top Ten mondiale degli ultrasessantacinquenni l'Italia occupa il secondo posto dopo il Giappone. In Europa, come indice di vecchiaia (popolazione $>65$ anni/popolazione $0-14$ anni x 100), l'Italia è al secondo posto dopo la Germania (dati ISTAT 2013).

L'invecchiamento della popolazione è associato a un progressivo maggior assorbimento delle risorse delle fasce di età dopo i 65 anni e a un sempre minor numero di popolazione attiva che finanzia la sanità. Vanno anche considerati gli effetti dei flussi migratori e dell'urbanizzazione con le conseguenti patologie prevalenti. Altre cause d'incremento della spesa sono le modificazioni epidemiologiche, l'avvento di nuovi farmaci e nuove tecnologie. Questo non è sempre giustificato. Ad esempio $1^{\prime} 80 \%$ dei nuovi farmaci immessi sul mercato sono copie (ad eccezione del prezzo) e solo il 2,5\% rappresenta un progresso terapeutico. La velocità di sviluppo della tecnica, che si autoalimenta, fa sì che le nuove apparecchiature diventino sempre più velocemente desuete, con una paranoica rincorsa al perennemente nuovo, che non sempre è giustificatamente meglio. Vanno, inoltre, tenuti in conto i costi sempre maggiori dovuti ai contenziosi medico-legali.

Il problema è quindi se si riuscirà, nonostante questo trend d'incremento della spesa, a mantenere sostenibile il sistema sanitario.

La sostenibilità è la caratteristica di un processo o di uno stato che può essere mantenuto a un certo livello indefinitamente. Lo sviluppo sostenibile è quello 
che soddisfa i bisogni del presente senza compromettere la possibilità delle generazioni future di soddisfare i propri (Rapporto Brunland WCED 1987). Il sistema di welfare degli anni'60-90 è stato insostenibile, perché ha compromesso la possibilità di soddisfare $i$ bisogni delle attuali e future generazioni.

Tra le proposte di soluzione a tale problematica già il 22 novembre 2001 la Conferenza Stato-Regioni aveva raggiunto l'intesa sul provvedimento del Governo di definizione dei Livelli Essenziali di Assistenza sanitaria (LEA) da garantire a tutti i cittadini. L'Ospedale abbandona definitivamente il suo ruolo di luogo di riferimento per qualsiasi problema di una certa rilevanza di natura sanitaria, e, spesso, socio-sanitaria, trasformandosi in un'organizzazione ad alto livello tecnologico deputata a fornire risposte assistenziali di tipo diagnostico - terapeutico a problemi di salute. Gravità e acuzie sono gli elementi cardine che ne caratterizzano la sfera di azione diagnostico-terapeutica.

Il disegno era quello di un riassetto strutturale e di una qualificazione tecnologica, con contemporanei programmi di sviluppo della rete dei servizi territoriali, nonché d'incisivi progetti per l'incremento del grado di appropriatezza. La realizzazione, però, di un disegno così ben costruito, è stata ampiamente inefficace, perché se da una parte non sono stati implementati i sistemi di sviluppo del territorio, per garantire l'erogazione su di esso dei livelli essenziali di assistenza, dall'altra questi ultimi sono stati usati per determinare l'inappropriatezza dell'attività degli ospedali. Ovvero, da parte della Pubblica Amministrazione si è rigorosamente attuato ciò che si doveva tagliare, ma non si è per nulla realizzata quella riqualificazione territoriale necessaria a garantire il welfare. Risultato: incremento della spesa, tagli lineari agli ospedali e riduzione quali/quantitativa della pubblica assistenza.

Nell'articolo di Nino Cartabellotta, presente in questa monografia, sono stati esaustivamente trattati gli aspetti strutturali del futuro del nostro Sistema Sanitario Nazionale. In tale articolo anch'egli sottolinea la grande incompiuta dei $L E A$ e il perpetuarsi delle ingerenze della politica partitica nella programmazione sanitaria. Si fa ampio riferimento al nuovo Patto per la Salute 2014-2016 intesa Stato-Regioni, recentemente approvato dal Ministero della Salute e dalle Regioni e si pone l'accento sui principali aspetti positivi. Il primo è che esso sembra aver messo in sicurezza il finanziamento del SSN, fissando le risorse destinate alla sanità pubblica sino al 2016. Tuttavia, il Ministero dell'Economia e delle Finanze non ha rinunciato alla precisazione salvo eventuali modifiche che si rendessero necessarie in relazione al conseguimento degli obiettivi di finanza pubblica e a variazioni del quadro macroeconomico. Il secondo grande aspetto di merito è di avere legittimato la rilevanza della clinical gover- nance (CG) come passaggio obbligato per salvare il nostro Sistema Sanitario Nazionale.

Nella seconda parte di queste conclusioni sul futuro della Medicina Interna sembra necessario evidenziare alcune preoccupazioni sull'attuazione di questi standard ospedalieri, peraltro così ben teorizzati. Anche nel Patto 2014-2016, vi sono da un lato misure restrittive e, dall'altro, provvedimenti di ampliamento e potenziamento dell'assistenza.

Tra i primi vi è una riduzione dei posti letto per acuti negli ospedali, questi scendono al di sotto di quattro $(3,7)$ per ogni mille abitanti, comprensivi di 0,7 (/1000 ab.) per la riabilitazione e la lungo degenza post-acuzie. Negli Ospedali il numero dei dipendenti amministrativi non dovrà superare il $7 \%$ di tutto il personale. Sono inoltre definiti, in senso restrittivo, i bacini di utenza dei presidi di primo e secondo livello e delle Aziende Ospedaliere e Ospedaliero/Universitarie, degli IRCS o ASL di grandi dimensioni, oltre che gli standard operativi minimi.

Questi tagli sono giustamente dettati dalla necessità di razionalizzare la spesa pubblica, migliorandone l'efficienza, adeguando il sistema assistenziale ai mutamenti sociali ed epidemiologici, che, come detto in apertura, vedono una società sempre più anziana, affetta da polipatologie, tutte croniche, con necessità di assistenza continuativa, con perdita di autonomie. La maggioranza delle famiglie non è in grado di erogare tale assistenza, con le conseguenti dimissioni difficili e frequenti e precoci re-ospedalizzazioni. A fronte di tali tagli, vengono prospettati maggiori investimenti nell'assistenza diretta (ammalati ad alta complessità assistenziale), incremento dei posti letto di Lungo Degenza, di Riabilitazione, implementazione delle Unità Alzheimer. Maggiore impegno e investimenti sul territorio, anche con l'istituzione di Ospedali di Comunità, che avrebbero lo scopo di assistere pazienti complessi, dimessi dagli Ospedali ancora bisognevoli di cure e cui la famiglia, per motivi sociali, economici, ambientali, non può prestare assistenza. La gestione di tali ospedali di comunità dovrebbe essere affidata ai Medici territoriali.

In quest'ambito potrebbe entrare la figura del futuro Medico Internista Ospedaliero. Questi potrebbe affiancarsi ai Medici del territorio nella gestione di tali strutture, allo scopo di garantire la continuità assistenziale e di trasmettere il suo know how sulla continuità di cura al Medico di Medicina Generale.

Questo è il primo aspetto evolutivo dell'Internista del futuro: una reale integrazione con il Territorio. Attualmente in Italia abbiamo un sistema di cure che funziona come un radar, cui il paziente appare per essere curato e scompare alla vista una volta stabilizzato. Sistema adeguato per le malattie acute, ma assolutamente carente per quelle croniche, per le quali serve invece un modello di assistenza diverso: occorre evi- 
tare non solo che le persone si ammalino, ma anche che chi è già malato vada incontro a ricadute, aggravamenti e disabilità. Un sistema, insomma, adatto a malattie che non guariscono e che devono essere seguite nel territorio adeguatamente attrezzato e integrato. Il paziente con patologie croniche ha bisogno di fiducia e di continuità assistenziale percepita come l'esistenza di un riferimento stabile e continuo. C'è bisogno di un grande lavoro sul versante culturale, professionale, organizzativo e operativo, che tenda a superare progressivamente l'autoreferenzialità che caratterizza ogni attore assistenziale, sia esso ospedaliero o territoriale. È necessario che si costruisca una rete integrata di attori riconoscibili, nella quale il paziente possa muoversi avendo riferimenti documentalmente affidabili e stabili, dei quali deve poter percepire il valore e la differenza all'interno di un disegno unitario.

L'altro principale aspetto del futuro internista si collega fortemente al primo ed è, appunto, la vera valorizzazione che l'attuale Patto per la Salute ha dato alla CG, unico strumento che potrebbe garantire: efficacia, appropriatezza (sia professionale sia organizzativa), efficienza e, quindi, sostenibilità.

Naturalmente la CG impone a tutti i professionisti sanitari sia si acquisire nuove competenze - non ancora previste dai programmi di formazione universitaria e specialistica - sia soprattutto di essere accountable in tutte le prescrizioni diagnostico-terapeutico-assistenziali, rinunciando ad autonomie professionali e interessi corporativi, oggi non più sostenibili. In quest'ambito la Medicina Interna ha una funzione portante, coordinando e completando il livello di complessità metaspecialistica e rivestendo la primaria missione di governo clinico.

In questo senso la FADOI ha un notevole vantaggio competitivo, avendo da tempo avviato e realizzato imponenti piani formativi nell'ambito del management in Sanità e dato il via a un progetto di valutazione della clinical competence, come ben si può evincere da altri articoli presenti in questo numero monografico.

Il futuro è, quindi, ormai vicino, sta a noi realizzarlo, il vero problema è che nel triangolo del cambiamento che vede ai suoi vertici: la conoscenza, le persone/po- polazione e il Governo/Istituzioni, noi possiamo influire solo sui due primi aspetti. Non possiamo, purtroppo, avere alcuna certezza sull'attuazione della riforma da parte delle Istituzioni. La vera preoccupazione è che anche l'attuazione di tale Patto segua il destino dei LEA, ovvero che i tagli dei posti letto, il ridimensionamento dei bacini di utenza, il rigore nel rispetto degli standard assistenziali, non vengano compensati dal maggiore investimento nell'assistenza diretta, nell'effettiva implementazione degli ospedali di comunità, in collaborazione con la Medicina Interna Ospedaliera. A noi rimarrà la certezza che si taglierà quanto promesso e il timore che non sarà concesso quanto prospettato per evitare un ulteriore crollo dell'assistenza, sia sotto il profilo della qualità che sotto quello dell'effettiva erogazione.

Potendo, quindi, contare con sicurezza solo sul nostro operato, è necessario realizzare i progetti di CG per cercare di mantenere il finanziamento della Sanità Pubblica. Evitare il razionamento, ovvero i tagli lineari attuati dall'alto, e tradurre in realtà una razionalizzazione, che deve essere concretizzata dal basso, da tutti noi operatori sanitari, attraverso un uso appropriato delle risorse. Evitare sovrautilizzo d'indagini e trattamenti, dare valore alla storia del paziente, la voce della vita, riappropriarsi della semeiotica fisica, ridare importanza all'esame obiettivo e su questa base richiedere gli esami appropriati.

Non ostinarsi a modificare la struttura, ma il modo di lavorare e di pensare (programmazione, coordinamento, trasparenza, accountability), focalizzarsi sui problemi e sui sistemi in grado di risolverli, mettere la persona al centro, gestire la conoscenza e il talento con la stessa attenzione con cui si gestiscono i soldi.

Non ritenere che il nuovo sia sempre meglio, in particolare riguardo ai farmaci e ai dispositivi medicali.

Ieri avevamo comportamenti dettati dalla saggezza clinica ma meno conoscenze e minore supporto tecnico. Oggi abbiamo molta più conoscenza e spropositato sviluppo tecnico, ma minore saggezza nel gestirli.

Se vogliamo garantirci il futuro del domani dobbiamo ritornare ai modi dell'ieri con le conoscenze e la tecnica dell'oggi. 


\section{LINEE GUIDA PER GLI AUTORI}

I Quaderni dell'Italian Journal of Medicine (Quaderni ITJM), costituiscono una collana supplementare solo online annessa alla rivista Italian Journal of Medicine contenente lavori solo in lingua italiana.

I Quaderni ITJM pubblicano:

- Monografie ad hoc su temi di particolare rilevanza scientifica a cura della Commissione FADOI Giovani, coordinata dalla Dr.ssa Paola Gnerre.

- Traduzioni in italiano di alcuni lavori pubblicati sui numeri standard dell'Italian Journal of Medicine, di particolare interesse per la comunità scientifica. La selezione è a cura dell'Editor-inChief, Prof. Roberto Nardi.

\section{STESURA DEI LAVORI}

I lavori dovranno essere redatti in modo conforme alle linee guida sotto riportate:

- I manoscritti devono essere scritti interamente in lingua italiana, su documento di Word, con carattere Times New Roman/Arial, dimensione 12, formato A4, interlinea doppia e margini $2,54 \mathrm{~cm}$. Parole totali: $\max 4000$; Sommario/Abstract: $\max 250$ parole; Bibliografia: $\min 40$ voci; Tabelle e Figure: $3 / 5$ totali (le tabelle non devono superare n. 1 pagina del documento in Word).

- La strutturazione del contenuto deve attenersi agli standard internazionali per la Rassegna (Review): i) Abstract riassuntivo dell'intero lavoro; ii) Introduzione al tema trattato; iii) Criteri e strumenti di ricerca (criteri di inclusione/esclusione, banche dati consultate, ...); iv) i successivi paragrafi devono illustrare le più recenti scoperte scientifiche nel settore; v) Conclusioni; vi) Bibliografia.

- La prima pagina deve riportare: i) titolo (in stampatello minuscolo), senza acronimi; ii) nome e cognome per esteso di ciascun autore; iii) affiliazione(i) di ciascun autore, numerate con numeri arabi; iv) eventuali ringraziamenti; v) nome e indirizzo postale completi dell'autore corrispondente, corredati da telefono, fax, e-mail; vi) da 3 a 5 parole chiave, separate da virgola. La seconda pagina può riportare: i) contributi degli autori, e.g. informazioni relative a contributi sostanziali delle persone coinvolte nello studio (http://www.icmje.org/\#author); ii) dichiarazione relativa a potenziali conflitti d'interesse; iii) ulteriori informazioni (e.g. fondi, esposizioni durante conferenze...).

- In caso di utilizzo di tabelle, queste devono essere tutte numerate con numeri arabi e citate nel testo in ordine consecutivo (e.g. NON nominare le tabelle come Tabella $1 \mathrm{~A}, 1 \mathrm{~B}, \ldots$ o $1.0,1.1, \ldots$ ). Le tabelle devono essere presentate in formato editabile. Ciascuna tabella deve essere corredata da una breve didascalia; in caso di abbreviazioni, riportare una nota a piè di CIASCUNA tabella che spieghi TUTTE le abbreviazioni presenti in ognuna.

- In caso di utilizzo di figure, queste devono essere inviate in formato .tiff o .jpg, allegate al manoscritto in singoli files, secondo le seguenti specifiche:

i) a colori (salvate in modalità CMYK): minimo 300 dpi di risoluzione;

ii) in bianco e nero: minimo 600 dpi di risoluzione;

iii) minimo $17,5 \mathrm{~cm}$ di larghezza.

Ciascuna figura deve essere corredata da una breve didascalia.

NB: In caso di Tabelle/Figure riprese e/o modificate da altri lavori già pubblicati, sarà cura degli autori accertarsi se tali materiali siano o meno coperti da copyright e procurarsi i permessi necessari per la riproduzione. Tali permessi dovranno essere allegati alla versione definitiva del lavoro. L'ufficio editoriale si riserva la facoltà di rimuovere Tabelle/Figure coperte da copyright, se sprovviste dei necessari permessi.

- In caso di utilizzo di abbreviazioni, la prima volta che esse sono citate è necessario scrivere per esteso la definizione+abbreviazione tra parentesi tonde [e.g. risonanza magnetica $(\mathrm{RMN})]$, a seguire si dovrà riportare solo l'abbreviazione (unica eccezione: nei titoli e nelle didascalie di tabelle e figure NON si utilizzano abbreviazioni).

\section{BIBLIOGRAFIA}

Le voci bibliografiche devono essere formattate secondo lo stile Vancouver.

Nella sezione Bibliografia, le voci bibliografiche devono essere numerate consecutivamente nell'ordine in cui appaiono per la prima volta nel testo (NON in ordine alfabetico) e, nel testo, devono essere indicate con numeri arabi in apice. Voci bibliografiche riferite a comunicazioni personali o dati non pubblicati devono essere incorporate nel testo e NON inserite tra le voci numerate [e.g. (Wright 2011, dati non pubblicati) o (Wright 2011, comunicazione personale)].

Le voci bibliografiche nella sezione Bibliografia devono tassativamente essere preparate come segue:

i) più di 3 autori, citare 3 autori, et al. Se il lavoro contiene solo 4 autori, citarli tutti e 4 ;

ii) titolo del lavoro in stampatello minuscolo;

iii) nome della rivista, senza punti, abbreviato secondo gli standard internazionali; in caso di dubbi sulla corretta abbreviazione, fare riferimento ai seguenti siti:

a. ISI Journal Abbreviations Index (http://library.caltech.edu/ reference/abbreviations/);

b. Biological Journals and Abbreviations (http://home.ncifcrf. gov/research/bja/);

c. Medline List of Journal Titles (ftp://ftp.ncbi.nih.gov/pubmed/J_Medline.txt);

iv) inserire l'anno di pubblicazione subito dopo il nome della rivista, seguito da punto e virgola;

v) NON inserire giorno o mese di pubblicazione;

vi) citare solo il volume, seguito dai due punti (NON citare il fascicolo tra parentesi);

vii) abbreviare le pagine, e.g. 351-8.

Per accertarsi di aver correttamente formattato le voci bibliografiche, confrontarle con le citazioni in PubMed (http://www.ncbi. nlm.nih.gov/pubmed).

Esempi (prestare attenzione anche alla punteggiatura):

Articolo standard su Rivista

Halpern SD, Ubel PA, Caplan AL. Solid-organ transplantation in HIV-infected patients. N Engl J Med 2002;347:284-7.

\section{Proceedings}

Christensen S, Oppacher F. An analysis of Koza's computational effort statistic for genetic programming. In: Foster JA, Lutton E, Miller J, Ryan C, Tettamanzi AG, eds. Genetic programming. EuroGP 2002: Proceedings of the 5th European Conference on Genetic Programming, 2002 Apr 3-5, Kinsdale, Ireland. Berlin: Springer; 2002. pp 182-91.

Articoli i cui autori sono Organizzazioni

Diabetes Prevention Program Research Group. Hypertension, insulin, and proinsulin in participants with impaired glucose tolerance. Hypertension 2002;40:679-86.

Libri

Murray PR, Rosenthal KS, Kobayashi GS, Pfaller MA. Medical microbiology. 4th ed. St. Louis, MO: Mosby; 2002. (CITAZIONE DEL LIBRO INTERO)

Meltzer PS, Kallioniemi A, Trent JM. Chromosome alterations in human solid tumors. In: Vogelstein B, Kinzler KW, eds. The genetic basis of human cancer. New York, NY: McGraw-Hill; 2002. pp 93113. (CITAZIONE DI UN CAPITOLO)

\section{MODALITÀ D'INVIO DEI LAVORI}

Monografie

Gli Autori dovranno fare riferimento alla Dr.ssa Gnerre (e-mail: pgnerre@yahoo.it) e agli eventuali altri referenti da lei designati, nelle prime fasi di stesura dei manoscritti, revisioni e correzioni. 


\section{QUADERNI}

\section{dell'Italian Journal \\ of Medicine}

La Dr.ssa Gnerre raccoglierà poi le versioni definitive dei lavori di ciascuna monografia e provvederà all'invio di tutti i materiali all'ufficio editoriale.

I lavori solo nella loro versione definitiva e approvata dalla Commissione FADOI Giovani dovranno pervenire all'ufficio editoriale già pronti per l'impaginazione e immediata pubblicazione (già corredati da eventuali permessi per la riproduzione di tabelle e immagini).

\section{Traduzioni}

Previo invito dell'Editor-in-Chief, gli Autori dovranno far pervenire all'ufficio editoriale la versione tradotta in italiano, al seguente indirizzo e-mail: paola.granata@pagepress.org

Il file in formato Word dovrà essere formattato secondo gli standard editoriali della rivista ufficiale ed essere già pronto per impagina-

\section{STAFF EDITORIALE}

Paola Granata, Journal Manager

paola.granata@pagepress.org

Selvaggia Stefanelli, Marketing Manager

marketing@pagepress.org

Claudia Castellano, Production Editor

Tiziano Taccini, Technical Support zione e immediata pubblicazione (corredato da eventuali permessi per la riproduzione di tabelle e immagini).

Si prega di inviare le eventuali tabelle in formato editabile e le figure in alta definizione secondo gli standard sopra riportati.

\section{NOTA PER GLI AUTORI}

I lavori pubblicati sui Quaderni ITJM non verranno indicizzati, ma saranno liberamente disponibili in un'apposita sezione del sito FADOI (http://www.fadoi.org/).

Gli Autori i cui lavori siano accettati per la pubblicazione sui Quaderni ITJM e che fossero interessati a vederli pubblicati anche sulla rivista ufficiale, dovranno sottomettere attraverso il sito dell'ITJM (www.italjmed.org) la versione (già tradotta) in inglese e redatta in modo conforme alle linee guida della rivista; seguiranno poi la procedura di selezione tramite peer review e, se accettati, saranno inseriti nel piano editoriale standard.

\section{QUADERNI - ITALIAN JOURNAL OF MEDICINE}

Tutti gli articoli pubblicati sui QUADERNI - Italian Journal of Medicine sono redatti sotto la responsabilità degli Autori. La pubblicazione o la ristampa degli articoli della rivista deve essere autorizzata per iscritto dall'editore. Ai sensi dell'art. 13 del D.Lgs 196/03, i dati di tutti i lettori saranno trattati sia manualmente, sia con strumenti informatici e saranno utilizzati per l'invio di questa e di altre pubblicazioni e di materiale informativo e promozionale. Le modalità di trattamento saranno conformi a quanto previsto dall'art. 11 del D.Lgs 196/03. I dati potranno essere comunicati a soggetti con i quali PAGEPress intrattiene rapporti contrattuali necessari per l'invio delle copie della rivista. Il titolare del trattamento dei dati è PAGEPress Srl, via Belli 7 - 27100 Pavia, al quale il lettore si potrà rivolgere per chiedere l'aggiornamento, l'integrazione, la cancellazione e ogni altra operazione di cui all'art. 7 del D.Lgs 196/03.
PUBBLICATO DA

PAGEPress Publications

via G. Belli 7

27100 Pavia, Italy

T. +39.0382 .1751762$

F: +39.0382 .1750481$

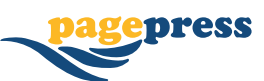

www.pagepress.org

info@pagepress.org
Pubblicato: dicembre 2014. 